volume 7

ISSUE 6

2019 agosto

Editor in Chief

Paola Gnerre

Supervisor Editor

Roberto Nardi

\title{
Rischio clinico in sanità e prevenzione dei rischi del paziente ricoverato in ospedale
}

Guest Editors: M. La Regina, E. Romano, S. Tardivo, E. Trinchero 
PRESIDENTE ELETTO

Dario Manfellotto, Roma, Italy

PAST PRESIDENT

Mauro Campanini, Novara, Italy

SEGRETARIO

Micaela La Regina, La Spezia, Italy

SEGRETARIO VICARIO

Andrea Montagnani, Grosseto, Italy

\section{STAFF DI SEGRETERIA}

\begin{tabular}{ll}
\hline Comunicazione & Claudia Tieri, Bari, Italy \\
Ricerca & Roberta Re, Novara, Italy \\
Formazione & Maurizia Gambacorta, Todi $(P G)$, Italy
\end{tabular}

TESORIERE

David Terracina, Roma, Italy

\section{STAFF DI TESORERIA}

Francesco D'Amore, Roma, Italy

PRESIDENTE FONDAZIONE FADOI

Mauro Campanini, Novara, Italy

COORDINATORE

Giuseppe Augello, Canicatti ( $A G)$, Italy

\section{DIPARTIMENTO PER LA RICERCA CLINICA FADOI}

Direttore Francesco Dentali, Varese, Italy

Supervisor per la Ricerca Giancarlo Agnelli, Perugia, Italy

DIPARTIMENTO PER LA FORMAZIONE

E AGGIORNAMENTO

Direttore Mauro Silingardi, Bologna, Italy

COORDINAMENTO FORMAZIONE

AREA CENTRO-NORD

Francesco Orlandini, La Spezia, Italy

COORDINAMENTO FORMAZIONE

AREA CENTRO-SUD

Generoso Uomo, Napoli, Italy

COORDINATORE COMMISSIONE GIOVANI

Flavio Tangianu, Oristano, Italy

MEMBRO FISM E RESPONSABILE

PER L'INNOVAZIONE IN MEDICINA INTERNA

Antonino Mazzone, Legnano (MI), Italy

ITALIAN JOURNAL OF MEDICINE

Editor in Chief Giorgio Vescovo, Padova, Italy
RESPONSABILE DEI

QUADERNI DELL'ITALIAN JOURNAL OF MEDICINE

Paola Gnerre, Savona, Italy

SUPERVISOR EDITOR DEI

QUADERNI DELL'ITALIAN JOURNAL OF MEDICINE

Roberto Nardi, Bologna, Italy

DELEGATO FADOI ITALIAN STROKE ORGANIZATION

E CONSULTA CARDIOVASCOLARE

Michele Stornello, Siracusa, Italy

\section{RAPPORTI CON EFIM}

Antonio Brucato, Bergamo, Italy

Gualberto Gussoni, Milano, Italy

Ombretta Para, Firenze, Italy

Giorgio Vescovo, Padova, Italy

RESPONSABILE SITO NAZIONALE

Salvatore Lenti, Arezzo, Italy

RESPONSABILE SISTEMA GESTIONE QUALITÀ

Franco Berti, Roma, Italy

RESPONSABILE CLINICAL COMPETENCE

Antonino Mazzone, Legnano (MI), Italy

RESPONSABILE AREA ECOGRAFIA

Francesco Cipollini, Ascoli Piceno, Italy

Marcello Romano, Catania, Italy

\section{RESPONSABILE MEDICINA DI GENERE}

Cecilia Politi, Isernia, Italy

\section{RESPONSABILE AREA NUTRIZIONE}

Roberto Risicato, Siracusa, Italy

Massimo Rondana, Pordenone, Italy

Luciano Tramontano, Praia a Mare (CS), Italy

RESPONSABILE AREA DI CLINICAL GOVERNANCE

Giovanni Iosa, Cesenatico ( $F C$ ), Italy

Stefano De Carli, Udine, Italy

\section{AREA SLOW MEDICINE}

Roberto Frediani, Chieri (TO), Italy

Luigi Lusiani, Castelfranco Veneto (TV), Italy

\section{AREA DOLORE}

Domenico Panuccio, Bologna, Italy

Giuseppe Civardi, Piacenza, Italy

\section{AREA CRITICA IN MEDICINA INTERNA}

Carlo Nozzoli, Firenze, Italy

\section{RESPONSABILE AGGIORNA FADOI}

Giuliano Pinna, Asti, Italy 


\section{Italian Journal of Medicine}

A Journal of Hospital and Internal Medicine

\section{PRESIDENTE FONDAZIONE}

Mauro Campanini, Novara, Italy

\section{COORDINATORE}

Giuseppe Augello, Canicattì ( $A G)$, Italy

\section{DIPARTIMENTO PER LA RICERCA CLINICA FADOI}

Direttore $\quad$ Francesco Dentali, Varese, Italy

Supervisor per la Ricerca Giancarlo Agnelli, Perugia, Italy

SEGRETERIA

Grazia Panigada, Pescia (PT), Italy

DELEGATO SIF

Francesco Rossi, Napoli, Italy

DELEGATO ANÍMO

Alberto Dal Molin, Novara, Italy

RESPONSABILE AREA MALATTIE CARDIOVASCOLARI

Paolo Verdecchia, Assisi (PG), Italy

\section{STAFF AREA MALATTIE CARDIOVASCOLARI}

Cecilia Becattini, Perugia, Italy

Pierpaolo Di Micco, Napoli, Italy

Fernando Gallucci, Napoli, Italy

Alessandro Squizzato, Varese, Italy

\section{RESPONSABILE AREA MALATTIE INFETTIVE}

Ercole Concia, Verona, Italy

\section{STAFF AREA MALATTIE INFETTIVE}

Anna Maria Azzini, Verona, Italy

Gianluca Giuri, Castelnovo ne'Monti (RE), Italy

Matteo Giorgi Pierfranceschi, Piacenza, Italy

Carlo Tascini, Pisa, Italy

\section{RESPONSABILE AREA MALATTIE RESPIRATORIE}

Leonardo Fabbri, Reggio Emilia, Italy

\section{STAFF AREA MALATTIE RESPIRATORIE}

Bianca Beghè, Reggio Emilia, Italy

Gaetano Cabibbo, Modica (RG), Italy

Francesco Corradi, Firenze, Italy

Francesco Ventrella, Cerignola $(F G)$, Italy

\section{RESPONSABILE AREA MALATTIE REUMATOLOGICHE}

Carlo Salvarani, Reggio Emilia, Italy

\section{STAFF AREA MALATTIE REUMATOLOGICHE}

Paola Faggioli, Legnano (MI), Italy

Laura Morbidoni, Senigallia (AN), Italy

Nicolò Pipitone, Reggio Emilia, Italy

Tito D'Errico, Napoli, Italy

\section{RESPONSABILE AREA MALATTIE METABOLICHE}

Roberto Vettor, Padova, Italy

\section{STAFF AREA MALATTIE METABOLICHE}

Tiziana Attardo, Agrigento, Italy

Giovanni Gulli, Savigliano (CN), Italy

Ada Maffettone, Napoli, Italy
Maurizio Nizzoli, Forli, Italy

RESPONSABILE AREA ORGANIZZATIVA

IN MEDICINA INTERNA

Antonio Greco, San Giovanni Rotondo (FG), Italy

\section{STAFF AREA ORGANIZZATIVA IN MEDICINA INTERNA}

Marco Candela, Jesi $(A N)$, Italy

Giovanni Mathieu, Pinerolo (TO), Italy

Valentino Moretti, San Daniele del Friuli (UD), Italy

Filomena Pietrantonio, Brescia, Italy

Elisa Romano, La Spezia, Italy

DIRETTORE DIPARTIMENTO PER LA FORMAZIONE

E AGGIORNAMENTO

Mauro Silingardi, Bologna, Italy

COORDINAMENTO DI AREA FORMATIVA CENTRO-NORD

Francesco Orlandini, La Spezia, Italy

COORDINAMENTO DI AREA FORMATIVA CENTRO-SUD

Generoso Uomo, Napoli, Italy

STAFF DIPARTIMENTO PER LA FORMAZIONE

E AGGIORNAMENTO

Roberto Frediani, Chieri (TO), Italy

Marco Grandi, Sassuolo (MO), Italy

\section{SEGRETERIA DIPARTIMENTO PER LA FORMAZIONE} E AGGIORNAMENTO

Luigi Magnani, Voghera (PV), Italy

\section{BOARD SCIENTIFICO}

Clelia Canale, Reggio Calabria, Italy

Fabrizio Colombo, Milano, Italy

Giuseppe De Matthaeis, Città Sant'Angelo (PE), Italy

Massimo Giusti, Torino, Italy

Luca Masotti, Cecina (LI), Italy

Andrea Montagnani, Grosseto, Italy

Nicola Mumoli, Livorno, Italy

Maurizio Ongari, Porretta Terme (BO), Italy

Ruggero Pastorelli, Colleferro (RM), Italy

Fulvio Pomero, Savigliano (CN), Italy

Roberto Risicato, Siracusa, Italy

Antonio Sacchetta, Treviso, Italy

Giancarlo Tintori, Pisa, Italy

COMMISSIONE TECNICHE E METODICHE INNOVATIVE DI FORMAZIONE E VERIFICA

Responsabile dell'Innovazione in Medicina Interna

Antonino Mazzone, Legnano (MI), Italy

STAFF

Francesco Dentali, Varese, Italy

Andrea Montagnani, Grosseto, Italy

Filippo Pieralli, Firenze, Italy

\section{DIRETTORE SCIENTIFICO FONDAZIONE FADOI}

Gualberto Gussoni, Milano, Italy 


\title{
QUADERNI - Italian Journal of Medicine
}

\author{
RISCHIO CLINICO IN SANITÀ E PREVENZIONE DEI RISCHI \\ DEL PAZIENTE RICOVERATO IN OSPEDALE \\ Guest Editors: M. La Regina, E. Romano, S. Tardivo, E. Trinchero
}

RISCHIO CLINICO IN SANITÀ

L'approccio sistemico alla gestione dei rischi in sanità .

E. Trinchero

Rischio clinico e sicurezza del paziente: presente e futuro . . . . . . . 7

S. Tardivo, M. Capasso

Rischio clinico: livelli di staffing infermieristico ed esiti correlati

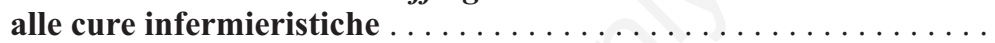

G. Pentella, L. Tesei

Il ruolo delle competenze non tecniche (non technical skill)

nella dinamica del rischio clinico

F. Moretti, M. Rimondini

Rischio clinico e carico di lavoro: alcuni spunti di riflessione

P. Gnerre, A. Fontanella, D. Montemurro, C. Palermo

TOP MANAGEMENT AZIENDALE E MEDICINA INTERNA:

LAVORARE INSIEME PER PROMUOVERE LA SICUREZZA DEL PAZIENTE

Il punto di vista di un Direttore Generale internista. .

G. Scannapieco, E. Croci

Il punto di vista di un Direttore Sanitario internista. . . . . . . . 43

F. Orlandini

Il punto di vista di un Direttore Sanitario igienista.......... 45

C. Bovo

Il punto di vista di un Direttore di Medicina Interna . . . . . . . . . 49

S. De Carli

\section{RISCHIO CLINICO E PREVENZIONE DEI RISCHI IN MEDICINA INTERNA}

La metà oscura del rischio clinico: i sinistri in medicina interna . . . 53

F. Corradi

La corretta identificazione del paziente

R. Gallo, F. Bertoncini, C. Gatta

Fase diagnostica: evitare misdiagnosi e ritardi

Sicurezza in terapia farmacologica.

E. Romano 3 


\section{QUADERNI - Italian Journal of Medicine}

Protezione dalle infezioni correlate all'assistenza . . . . . . . . . . . 85

A. Maffettone, C. Peirce, S. Di Fraia

Safe Handover .

R. Rapetti

Prevenzione dei rischi in Medicina Interna, casi particolari: atti autolesivi, vagabondaggio, intrappolamento nel paziente ospedalizzato

R. Nardi, D. Tirotta, G. Pinna, G. Pirini

Gestione ricoveri in appoggio e rischio clinico . . . . . . . . . . . . 105

M. La Regina, F. Guarneri, T. Bellandi, R. Tartaglia, A. Brucato, D. Manfellotto

Innovazioni organizzative in Medicina Interna per la sicurezza del paziente: dall'Ambulatorio Integrato Diagnostico-Terapeutico alla Sezione di Decisione Clinica .

R. Castello, A. Carli

Ossigenoterapia.

M. La Regina

Ventiloterapia

M. La Regina

Strumenti per una gestione sicura delle procedure invasive 121

M. La Regina

La prevenzione del tromboembolismo venoso .

F. Pomero, E. Nicola, P. Salomone, R. Risso

Sicurezza nella gestione della terapia anticoagulante orale

M. La Regina, F. Pomero

Strumenti per una gestione sicura delle terapie inalatorie.

C. Tieri

Strumenti per una gestione sicura della terapia ipoglicemizzante. . . 135 T.M. Attardo

Strumenti per una gestione sicura delle allergie a farmaci e delle interazioni farmacologiche.

T.M. Attardo

Mobilizzazione dei pazienti

G. Querci, A. Spadaro 


\title{
L'approccio sistemico alla gestione dei rischi in sanità
}

\author{
Elisabetta Trinchero \\ CeRGAS - Government, Health and Not for Profit - SDA Bocconi School of Management, Milano, Italia
}

\section{Introduzione}

Cosa hanno in comune il terremoto che ha colpito nel 2009 l'Ospedale San Salvatore dell'Aquila, l'incendio scoppiato nel 2013 in un ospedale psichiatrico di Mosca che ha provocato la morte di 36 pazienti e di due membri dello staff, e un problema amministrativo di Ryanair, che ha causato nel 2017 la cancellazione di oltre 2000 voli?

Si tratta di eventi, che seppur non comparabili per la gravità degli effetti, possono essere classificabili come crisi aziendali. Infatti, con crisi aziendale viene definito in letteratura un evento poco probabile ma di grande impatto che minaccia la sopravvivenza dell'azienda ed è caratterizzato da una grande ambiguità nelle cause, negli effetti e nelle modalità di risoluzione, nonché dalla convinzione che le decisioni debbano essere prese rapidamente. ${ }^{1}$ La crisi può essere tipizzata in base al principale fattore scatenante, identificando così crisi conseguenti a disastri naturali, al fallimento della tecnologia, a danni causati dall'azienda a terzi, a danni causati da terzi all'azienda, ad errori gestionali, ad atti di violenza sul personale, a fuga di informazioni sensibili ed infine ad attacchi terroristici. Le cause della crisi possono essere sia endogene sia esogene, a seconda della controllabilità dei fattori scatenanti la crisi da parte della azienda. ${ }^{2}$

Per quanto riguarda la gestione della crisi, le fasi chiave dell'evoluzione e della risposta alle crisi sono tipicamente le seguenti: i) il rilevamento dei segnali; ii) la preparazione e la prevenzione degli interventi; iii) il contenimento e il controllo dei danni; iv) il re-

Corrispondente: Elisabetta Trinchero, CeRGAS - Government, Health and Not for Profit - SDA Bocconi School of Management, Via Bocconi 8, room 512, Milano, Italia.

Tel.+39.02.5836.2277 - 6886; Fax.+39.02.5836.6893.

E-mail: elisabetta.trinchero@unibocconi.it

Articolo pubblicato secondo la Creative Commons Attribution NonCommercial 4.0 License (CC BY-NC 4.0).

${ }^{\circ}$ Copyright: the Author(s), 2019

Licensee PAGEPress, Italy

QUADERNI - Italian Journal of Medicine 2019; 7(6):1-6 cupero delle attività; e v) l'apprendimento organizzativo. ${ }^{3,4} \mathrm{Si}$ tratta di azioni che avvengono dopo che l'evento è accaduto e sono mirate al contenimento della magnitudo degli impatti della crisi sulla vita dell'azienda: impatti che possono spaziare da una interferenza con le normali attività operative (e dunque influire sulla cosiddetta business continuity), ad un effetto negativo, anche a lungo termine, sulla reputazione e sull'immagine dell'organizzazione, fino alla messa in discussione (reale o percepita) della sopravvivenza stessa dell'organizzazione.

Tra i fattori che influenzano la capacità di successo nella gestione di una crisi ricordiamo la presenza di un rodato team di crisis management, la possibilità di fare affidamento su di un piano di crisis management e di linee guida testate per la comunicazione con i media, e soprattutto una solida relazione antecedente alla situazione di crisi con gli stakeholders interni ed esterni all'azienda. ${ }^{5}$

Ma il vero fattore di critico di successo per una efficace gestione di una crisi è la possibilità di fare affidamento su di una positiva cultura della sicurezza a livello aziendale. ${ }^{6}$ Un'utile definizione di cultura della sicurezza viene dall'Advisory Committee on the Safety of Nuclear Installations. ${ }^{7}$ Tale definizione, infatti, è quella maggiormente accreditata a livello internazionale in tutti i settori di attività, compresa la sanità. L'ACSNI definisce la cultura della sicurezza di un'azienda come il prodotto di valori, attitudini, percezioni, competenze e comportamenti individuali e di gruppo, che determinano l'impegno, lo stile e il livello del sistema aziendale di gestione della sicurezza. Le aziende con una positiva cultura della sicurezza sono caratterizzate da processi di comunicazione che si basano sulla fiducia reciproca, da percezioni sulla rilevanza della sicurezza condivise e dalla fiducia nell'efficacia delle misure di prevenzione. Per valutare la cultura della sicurezza di una azienda sanitaria, un framework di analisi efficace è quello messo a punto da Dominic Cooper nel 2000. ${ }^{8}$ Tale modello presenta l'indiscusso vantaggio non solo di essere robusto da un punto di vista teorico, ma di fornire anche la strada per rendere possibile l'operativizzazione del concetto di cultura della sicurezza, che altrimenti rischia di rimanere una dimensione astratta. Il framework di analisi propone di focalizzare l'attenzione: i) sugli aspetti 
psicologici legati al tema della sicurezza (e quindi di come le persone si percepiscono), in termini di clima della sicurezza dell'azienda, e quindi di valori attitudini e percezioni individuali e di team; ii) sui comportamenti legati al tema della sicurezza (e quindi di ciò che le persone fanno), in termini di atteggiamenti e azioni del personale; iii) sugli aspetti situazionali legati al tema della sicurezza (e quindi di ciò che l'azienda mette a disposizione), in termini di politiche, procedure, regolamenti, struttura organizzativa e sistemi di governo.

Inoltre, si ritiene che la Social Exchange Theory ${ }^{9,10}$ abbia fornito un utile inquadramento teorico per misurare l'influenza del management delle aziende sanitarie sulla cultura della sicurezza. Ciò ha permesso di cogliere la relazione tra dimensioni quali la qualità della relazione con la direzione e il grado di coinvolgimento individuale e aspetti psicologici e comportamentali collegati alla cultura della sicurezza. ${ }^{11,12}$

\section{Approccio sistemico alla gestione dei rischi di un'azienda sanitaria}

Come dunque un'azienda, e nel nostro caso una azienda sanitaria, può attrezzarsi per intercettare e possibilmente disinnescare gli eventi che possano minarne la capacità di raggiungere $\mathrm{i}$ suoi obiettivi istituzionali e dunque di diagnosi, assistenza e riabilitazione della sua popolazione di pazienti?

L'invito è quello di superare la logica frammentaria, discontinua e reattiva di gestione dei rischi a canne d'organo, che induce attualmente le aziende sanitarie a sviluppare funzioni ad hoc per le diverse tipologie di rischio e di considerare in modo sistemico e sistematico nelle decisioni strategiche i rischi che possono impattare sulla vita dell'azienda sanitaria. ${ }^{13} \mathrm{Si}$ tratta dunque di introdurre nelle aziende sanitarie un processo strategico e trasversale progettato per identificare gli eventi avversi potenziali e per gestire i rischi che possono impattare sull'organizzazione al fine di fornire una ragionevole garanzia del raggiungimento dei suoi obiettivi. Per raggiungere questo risultato è necessario che tale processo sia influenzato non solo dall'alta direzione ma anche dai direttori di dipartimento, di area e di unità operativa, e dai professionisti che operano in prima linea. ${ }^{14}$

Il framework logico di massima da cui partire, e che necessariamente deve essere declinato per tenere in considerazione le specificità delle aziende sanitarie, è quello del cosiddetto Enterprise Risk Management (ERM) ${ }^{15}$ Con ERM si definisce il processo attraverso cui un'azienda anticipa, previene e risponde alle situazioni di incertezza organizzativa e ambientale associate al perseguimento degli obiettivi strategici. ${ }^{16}$ Più specificamente è il processo attraverso cui un'azienda definisce proattivamente le tipologie e i livelli di ri- schio che intende assumersi per il raggiungimento degli obiettivi di medio e lungo periodo. Si tratta dunque di un approccio integrato, olistico e sistemico per la gestione strategica dei rischi che supera l'approccio a compartimenti stagni di gestione separata delle differenti tipologie di rischio. In alcune aziende l'ERM si esplica in una vera e propria funzione aziendale.

Per inquadrare le caratteristiche dell'ERM, occorre fare una breve introduzione su cosa debba intendersi per rischio e per gestione del rischio. L'accezione di rischio fornita dalla norma UNI ISO 9001/2015 è molto ampia e attiene a tutto ciò che possa impattare sulla continuità dei processi aziendali (cosiddetta $b u$ siness continuity). L'approccio alla gestione del rischio è fornito dalla norma UNI ISO 31000/2018 e riguarda quell'insieme di attività messe in atto dall'azienda al fine di evitare, prevenire o ridurre gli eventi rischiosi a cui può essere soggetta. Il processo di gestione dei rischi si svolge per fasi successive: definizione del contesto, identificazione dei rischi, analisi e valutazione dei rischi, trattamento dei rischi (con la definizione delle priorità e azioni di mitigazione, accettazione $\mathrm{o}$ trasferimento dei rischi), comunicazione, monitoraggio e verifica delle azioni intraprese.

\section{Analisi dei rischi di un'azienda sanitaria}

Ma quali sono i rischi che attraversano una azienda sanitaria? Vi sono diverse classificazioni di rischi in letteratura che dipendono dal framework di risk management utilizzato. ${ }^{17}$ Le principali categorie di rischio che possono interessare un'azienda sanitaria, sia essa pubblica o privata, elaborate partendo dal framework dell'ERM, comprendono i rischi strategici, i rischi operativi, i rischi finanziari e i rischi di conformità. ${ }^{15}$ Partendo da queste categorie di rischio, ogni realtà sanitaria può costruirsi nel tempo un inventario dei rischi che affronta, definendone la priorità in termini di probabilità di accadimento, impatto e rilevabilità. ${ }^{18}$

\section{Rischi strategici}

Rientrano nella macro-categoria dei rischi strategici quelli correlati alla vision dell'azienda e ai suoi valori fondanti; al sistema di governance e alla struttura organizzativa dell'azienda; alla pianificazione strategica; a fusioni e acquisizioni (ad esempio correlati alle recenti azioni di riassetto delle aziende sanitarie in molte regioni italiane); alla gestione dei rapporti con gli investitori; alla concorrenza; ad eventuali modifiche delle preferenze o degli stili di vita dei clienti (ad esempio rischi correlati all'invecchiamento della popolazione, che richiedono un ripensamento del sistema di offerta); agli investimenti in mercati emergenti (ad esempio la medicina estetica o il sistema del wellness). 


\section{Rischi finanziari}

Rientrano nella macro-categoria dei rischi finanziari quelli correlati alla volatilità del tasso di interesse e delle valute estere; alle politiche e alle stime contabili; ai sistemi di controllo interno; alla strategia fiscale; alla gestione del credito e della liquidità.

\section{Rischi operativi}

Rientrano nella macro-categoria dei rischi operativi quelli correlati all'attività di produzione dei beni e dei servizi aziendali (il cosiddetto core business). In particolare, si tratta di rischi legati alla ricerca e sviluppo, all'introduzione di nuovi prodotti e servizi; ad azioni di promozione dei servizi; all'attività di budgeting; alla disponibilità delle risorse necessarie per l'erogazione dei prodotti e servizi; alla gestione dei fornitori; alla gestione delle scorte; alla gestione della tecnologia; alla gestione delle risorse umane; alla $c y$ bersecurity e alla business continuity.

Tra i rischi operativi tipici delle aziende sanitarie ricordiamo il rischio clinico, definito dall'IOM nel 1999 quale possibilità che un paziente subisca un danno o disagio involontario, imputabile alle cure sanitarie, che causa un prolungamento del periodo di degenza, un peggioramento delle condizioni di salute o la morte. ${ }^{19}$ Per superare la visione ospedalo-centrica della precedente definizione, nel 2007 l'NHS britannico lo ha ridefinito come la probabilità di un outcome avverso in seguito a un processo diagnostico, terapeutico o assistenziale. ${ }^{20}$ Gli strumenti prospettici e retrospettivi di identificazione del rischio clinico sono tipicamente: l'esecuzione di audit e di Mortality \& Morbidity review (M\&M review) ${ }^{21}$ l'analisi della documentazione clinica, ad esempio secondo la tecnica nota come Trigger Tool:22 l'esecuzione di walk around; l'utilizzo di tecniche di flow-charting e analisi di processo quali Failure Mode and Effect Analysis (FMEA), ${ }^{23}$ Root Cause Analysis (RCA) e Probabilistic Risk Assessment (PRA); l'analisi di indicatori di performance; l'analisi dei dati provenienti dal sistema aziendale di segnalazione degli eventi avversi (Incident reporting system) e di raccolta dei reclami e dei sinistri. ${ }^{24}$

Da una ricognizione condotta inizialmente nel 2006 nelle aziende sanitarie pubbliche italiane, ${ }^{25}$ e successivamente ripresa nel $2012^{26}$ e nel 2018 in occasione della presente pubblicazione, la funzione di gestione del rischio clinico sembra essere tipicamente incardinata in una unità semplice o complessa variamente denominata a seconda del contesto regionale, collocata più frequentemente in staff alla direzione generale che alla direzione sanitaria, generalmente isolata dalle altre aree aziendali proposte alla gestione degli altri rischi. La dotazione di personale è molto variabile da azienda ad azienda sia per numero che per composizione. Troviamo infatti medici, infermieri, farmacisti, ingegneri clinici, psicologi, laureati in economia. È sulle attività e processi rilevanti per le politiche aziendali su cui la funzione di gestione del rischio clinico ha un'effettiva influenza che la risposta è quasi unanime: sembra che la maggioranza degli sforzi sia assorbita dall'organizzazione e in qualche caso erogazione diretta di interventi di formazione, seguita dalla promozione di iniziative di valutazione del rischio clinico ed infine da una seppur in crescita ancora residuale attività di project management di iniziative di innalzamento della sicurezza dei pazienti.

Per quanto riguarda la gestione dei rischi legati all'uso delle tecnologie sanitarie, sempre più complesse e sofisticate, è fondamentale la presenza di un efficace programma aziendale che valuti le vulnerabilità e i requisiti di conformità, che preveda la collaborazione e il coinvolgimento di ingegneri clinici, esperti di gestione del rischio, medici, infermieri, tecnici sanitari e altri stakeholder chiave. ${ }^{27}$ In particolare, per mitigare le principali minacce legate all'uso delle tecnologie sanitarie è opportuno introdurre e mantenere aggiornate: ${ }^{27}$ i) le misure di sicurezza fisico-ambientali (quali ad esempio porte taglia fuoco e distanza di sicurezza o schermature tra device); ii) le misure di sicurezza tecnico-amministrative (quali ad esempio sistemi di privilegi di accesso, controlli ridondanti, backup, reti isolate e software per eliminare le vulnerabilità note); iii) le competenze del personale (attraverso formazione cadenzata dedicata); iv) le linee guida di utilizzo e di monitoraggio (ad esempio per gli interventi di collaudo, di calibrazione, di manutenzione programmata).

Vale la pena ricordare che esistono enti, come ad esempio l'ECRI Institute (https://www.ecri.org/about/), che da anni mettono a diposizione di aziende sanitarie pubbliche e private, agenzie, ministeri della salute, associazioni e istituti di accreditamento informazioni utili alla protezione dei pazienti da pratiche e tecnologie mediche non sicure e inefficaci.

Per quanto riguarda i rischi connessi alle gestione delle persone, sul podio per potenzialità di influenzare le performance aziendali ritroviamo i rischi legati alla capacità dell'azienda di non farsi sfuggire i professionisti migliori, di garantire la presenza delle competenze fondamentali e una leadership efficace. ${ }^{28}$ Paradossalmente la selezione del personale viene considerata l'attività meno rischiosa, perché un errore nella selezione può essere corretto con politiche di inserimento e di sviluppo professionale adeguate, mentre anche il miglior processo di selezione del personale può essere invalidato da una gestione delle persone che ne distrugga la professionalità e la motivazione, magari attraverso l'assegnazione di obiettivi impossibili da raggiungere (cosiddetto work harassment). ${ }^{29}$

È infatti basilare la capacità della leadership di potenziare nei professionisti il capitale psicologico quale risorsa individuale fondamentale per il perseguimento 
degli obiettivi personali, professionali e aziendali. ${ }^{30} \mathrm{Si}$ tratta di caratteristiche quali l'assertività, intesa come confidenza di raggiungere un obiettivo; la speranza, intesa come forza e volontà nel raggiungere un obiettivo; l'ottimismo, inteso come aspettativa positiva rispetto ai risultati; e la resilienza, intesa quale capacità di affrontare le avversità. Sono caratteristiche individuali senza le quali diviene impossibile sviluppare nei professionisti le competenze per la sicurezza dei pazienti, quali quelle di saper contribuire alla cultura delle sicurezza, di saper lavorare in team interprofessionali, di saper comunicare efficacemente, di saper gestire i rischi, di saper ottimizzare i fattori umani e ambientali, di saper riconoscere, gestire e segnalare gli eventi avversi. ${ }^{31}$

Per quanto riguarda la gestione dei rischi connessi alla cybersecurity in sanità, emerge la necessità di investimenti strutturali per l'innalzamento della sicurezza delle infrastrutture e per l'aggiornamento delle competenze del personale che opera nelle unità di IT per garantire una capacità di risposta tempestiva ed efficiente alle minacce. Allo scopo di tutelare la sicurezza dei dati aziendali è fondamentale procedere alla classificazione di dati sensibili e alla creazione di strategie di protezione dei dati del paziente, nonché al coinvolgimento e alla responsabilizzazione degli utilizzatori del sistema (medici, infermieri, tecnici sanitari, personale amministrativo) sulle minacce e sugli strumenti di prevenzione. Inoltre è indispensabile la tenuta del sistema di gestione delle autorizzazioni e di protezione da accessi indesiderati per la difesa da minacce derivanti dall'uso di medical device e dei dispositivi mobili (cosiddetto Internet of Things, IoT) [Con IoT si intende generalmente l'insieme dei device, diversi dai computer, che si connettono ad internet, e che pertanto possono veicolare minacce alla sicurezza dei dati aziendali.]. ${ }^{32}$

\section{Rischi di conformità}

Rientrano nella macro-categoria dei rischi di conformità i rischi di frode, di corruzione e correlati al conflitto di interessi; i rischi legali correlati alla conformità alle norme nazionali e regionali, alla regolamentazione fiscale, all'adeguatezza delle procedure amministrative e dei contratti; ${ }^{33}$ i rischi legati all'impatto ambientale (ad esempio correlati alla gestione dei rifiuti speciali); i rischi correlati alla salute e alla sicurezza dei dipendenti e dei visitatori (rientrano in questa categoria ad esempio il rischio di caduta dei famigliari di un paziente, o il rischio di furto di un oggetto appartenente ad un paziente ricoverato).

Si può inserire in questa macro-categoria anche il rischio di condotta, rischio legato all'offerta inappropriata di servizi ${ }^{34}$ (come ad esempio la proposta ed erogazione al paziente di servizi sanitari inappropriati, inutili o addirittura dannosi).

Si ricorda infine che anche l'informativa agli stakeholder dei rischi che un'azienda sanitaria affronta è un tema oggetto di attenzione da parte del legislatore. Ad esempio, la legge 24/2017 (Disposizioni in materia di sicurezza delle cure e della persona assistita, nonché in materia di responsabilità professionale degli esercenti le professioni sanitarie, nota come Legge Gelli) all'art 4 (Trasparenza dei dati) chiede alle aziende sanitarie pubbliche e private la presentazione della documentazione sanitaria da parte degli interessati aventi diritto, nonché la pubblicazione sul sito internet aziendale dei dati relativi a tutti i risarcimenti erogati nell'ultimo quinquennio.

Sempre a proposito dell'informativa agli stakeholder dei rischi, si riportano i risultati di una ricerca che ha interessato le aziende sanitarie pubbliche italiane, attraverso l'analisi di 672 siti web di Istituti di Ricovero e Cura a Carattere Scientifico (IRCCS), Aziende Ospedaliere (AO), Aziende Ospedaliere Universitarie (AOU), Presidi Ospedalieri (PO) alla ricerca di informazioni pubblicate relativamente al rischio che i pazienti contraggano infezioni nosocomiali. Alla data di osservazione, una azienda su tre riportava sul proprio sito web informazioni relative alle misure di prevenzione delle infezioni nosocomiali in generale e all'igiene delle mani in particolare, mentre il $6 \%$ delle aziende analizzate fornivano i risultati della sorveglianza delle infezioni nosocomiali. Sebbene il $64 \%$ dei siti web riportassero informazioni rispetto al rischio, il $54 \%$ presentava un dato aggiornato ai 6 mesi precedente. Il 19\% dei siti web presentava una sezione del sito dedicata alle infezioni nosocomiali, e mediamente occorrevano tra tre a quattro passaggi per arrivarvi. Nel $2 \%$ dei casi le informazioni sulle infezioni nosocomiali erano disponibili in homepage. Sebbene nel $98 \%$ dei casi fosse possibile inviare una mail per chiedere informazioni, in nessun sito era disponibile un forum di discussione sul tema. ${ }^{35}$

\section{Rischi di sostenibilità e rischi reputazionali}

In alcune classificazioni si trovano anche le categorie dei rischi di sostenibilità e dei rischi reputazionali, ${ }^{36}$ sebbene possano essere raggruppate nelle categorie di rischio precedentemente elencate. Infatti, le implicazioni sulla reputazione dell'azienda sanitaria sono spesso conseguenza di un altro tipo di rischio. Esempi molto diversi tra loro per la gravità degli effetti, ma che hanno tra gli altri anche un impatto negativo sull'immagine dell'azienda sanitaria possono essere un evento sentinella quale la morte di un paziente derivante da errori nella terapia farmacologica oppure l'arresto di un dipendente per frode o corruzione. Nel primo caso si tratta di un evento ascrivibile al rischio clinico nella macro-categoria dei rischi operativi, mentre nel secondo caso si tratta di rischio di frode e corruzione ascrivibile alla macro-categoria dei rischi di conformità, ma l'effetto sulla popolazione sarà in entrambi i casi una perdita di fiducia della popolazione. 
In sintesi, per la gestione dei rischi di una azienda sanitaria emerge come sia fondamentale il supporto della direzione aziendale e dei direttori delle unità operativa e come sia necessario intervenire in modo integrato e coordinato: i) sia sulla struttura organizzativa, attraverso l'istituzione aziendale di una funzione aziendale di governo dei rischi; ii) sia sui meccanismi operativi di funzionamento, attraverso: 1'implementazione di buone pratiche, raccomandazioni e linee guida; l'attivazione di progetti e processi trasversali; la realizzazione di un efficace sistema informativo e di reporting e l'investimento nella gestione del personale in termini di motivazione, formazione continua, presidio dei carichi di lavoro, job description; iii) sia sulla cultura aziendale della sicurezza.

\section{Bibliografia}

1. Pearson CM, Clair JA. Reframing crisis management. Acad Manage Rev 1998;23:59-76.

2. Lerbinger O. The Crisis Manager: Facing Risk and Responsibility. Mahwah, NJ: Lawrence Erlbaum Associates; 1997.

3. Pearson CM, Mitroff II. From crisis prone to crisis prepared: a framework for crisis management. Executive 1993;7:48-59.

4. Schneider SK. Governmental response to disasters: The conflict between bureaucratic procedures and emergent norms. Publ Admin Rev 1992;52:135.

5. Wise K. The Oxford Incident: organizational culture's role in an anthrax crisis. Publ Relat Rev 2003;29:46172.

6. Brusoni M, Deriu PL, Panzeri C, Trinchero E. Un metodo di indagine sulla safety culture per la sicurezza dei servizi sanitari in Italia. Mecosan 2009;69:63-85.

7. ACSNI. ACSNI study group on human factors: Organizing for safety. HSE, editor. London: HMSO; 1993.

8. Cooper D. Towards a model of safety culture. Safety Sci 2000;36:111-36.

9. Gouldner AW. The norm of reciprocity: A preliminary statement. Am Sociol Rev 1960;25:161-78.

10. Blau P. Exchange and power in social life. New York, NY: John Wiley \& Sons; 1964.

11. Trinchero E, Farr-Wharton B, Brunetto Y. A social exchange perspective for achieving safety culture in healthcare organizations. Int J Publ Sect Manage 2019;32:142-56

12. Trinchero E, Farr-Wharton B, Brunetto Y. Workplace Relationships, Psychological Capital, Accreditation and Safety Culture: a new Framework of Analysis within Healthcare Organizations. Publ Organ Rev 2019;19:139.

13. Brusoni M, Trinchero E, Vescia M. La gestione del rischio in sanità: elementi organizzativi e gestionali. In: Aleo S, De Matteis R, Vecchio G, eds. La responsabilità in ambito sanitario. Padova: CEDAM; 2014.

14. Trinchero E. Rischi in sanità: è anche un problema di management. Econ Manage 2008;1:37-8.

15. COSO. Enterprise Risk Management: Integrating with Strategy and Performance; 2017.

16. Monahan G. Enterprise Risk Management: A methodology for achieving strategic objectives. New York, NY: John Wiley \& Sons; 2008.
17. Verbano C, Turra F. A human factors and reliability approach to clinical risk management: Evidence from Italian cases. Safety Sci 2010;48:625-39.

18. Brusoni M, Trinchero E. Responsabilità sociale e riduzione del rischio in sanità: il ruolo dei sistemi di accreditamento. In: Borgonovi E, Rusconi G, eds. La responsabilità sociale delle istituzioni di pubblico interesse. Milano: Franco Angeli; 2008.

19. Kohn LT, Corrigan J, Donaldson MS. To err is human: Building a safer health system. Washington, DC: National Academy Press; 1999.

20. Brusoni M, Trinchero E. Sistemi di assessment del rischio tra azienda sanitaria e settore assicurativo. Mecosan. 2008;66:89-101.

21. Rappini V, Trinchero E, Zavattaro F. Lo sviluppo dell'internal auditing in sanità. Tre esperienze d'eccellenza a confronto. Econ Manage. 2011;6:63-80.

22. Griffin FA, RK. R. IHI Global Trigger Tool for Measuring Adverse Events (Second Edition). Cambridge, MA: Institute for Healthcare Improvement; 2009.

23. Canitano S, Ghirardini A, Migliazza M, Trinchero E. Risk management, strumenti e cultura organizzativa per il governo della patient safety: dalla teoria alla pratica. Mecosan 2010;76:83-101.

24. Bonetti M, Cirillo P, Musile Tanzi P, Trinchero E. An Analysis of the Number of Medical Malpractice Claims and Their Amounts. PLoS One 2016;11:e0153362.

25. Brusoni M, Cosmi L, Trinchero E. Prospettive regionali per la gestione del rischio clinico: una prima indagine esplorativa. In: Anessi Pessina E, Cantù E, eds. L'aziendalizzazione della sanità in Italia Rapporto OASI 2006. Milano: Egea; 2006.

26. Brusoni M, Trinchero E, Marazzi L, Partenza I. Gestione, ritenzione e assicurazione del rischio: alla ricerca di una prospettiva integrata. In: Cantù E, editor. L'aziendalizzazione della sanita in Italia Rapporto Oasi 2012. Milano: Egea; 2012.

27. Grimes SL. Evolution of a Risk-Based Approach to Effective Healthcare Technology Management. Biomed Instr Technol 2015;49:34-42.

28. Meyer M, Roodt G, Robbins M. Human resources risk management: Governing people risks for improved performance. SA J Hum Resour Manage 2011;9:1.

29. Trinchero E, Farr-Wharton B, Borgonovi E. Relationships with managers and harassment: the Italian nurse experience. Public Money Manage 2017;37:341-8.

30. Luthans F, Youssef CM, Avolio BJ. Psychological capital. New York: Oxford University Press; 2007.

31. Accreditation Canada. Building a stronger health system through leadership. Ottawa, ON: Accreditation Canada; 2014.

32. Cabrera E. Health Care: Cyberattacks and How to Fight Back. J Health Care Compl 2016;18:27-30.

33. Sax J, Torp SS. Speak up! Enhancing risk performance with enterprise risk management, leadership style and employee voice. Manage Decis 2015;53:1452-68.

34. McConnell P. Operational Risk: A Forgotten Case Study. J Operat Risk 2018 [In press].

35. Gallone MS, Tafuri S, Preziosa VP, et al. How Italian hospital Web sites communicate risk management strategies: the case of hospital-acquired infections. Am J Infect Control 2014;42:813-4.

36. Gatzert N, Schmit J. Supporting strategic success through enterprise-wide reputation risk management. 2016;17:26-45. 


\title{
Rischio clinico e sicurezza del paziente: presente e futuro
}

\author{
Stefano Tardivo, Mario Capasso \\ Dipartimento di Diagnostica e Sanità Pubblica, Università di Verona, Italia
}

\section{Il concetto di errore in sanità}

\section{Basi teoriche e dimensioni del fenomeno}

La sicurezza del paziente rappresenta una dimensione fondamentale della qualità delle cure. ${ }^{1}$ Essa consiste nell'evitare, nel prevenire e nel mitigare eventi avversi o danni provocati dalla stessa assistenza sanitaria. $^{2}$

Come sottolineato da Vincent, ${ }^{3}$ la sicurezza del paziente viene talvolta assimilata alla prevenzione degli errori, definiti dall'Organizzazione Mondiale della Sanità (OMS) come fallimenti nell'esecuzione di un'azione pianificata in modo corretto oppure applicazioni corrette di un piano errato. ${ }^{4}$ In termini semplici, si verifica un errore quando qualcuno sta cercando di fare la cosa giusta, ma, in realtà, fa la cosa sbagliata, ${ }^{5}$ dando luogo ad una deviazione non intenzionale dall'obiettivo desiderato (ad es. una diagnosi sbagliata o la prescrizione di una terapia inappropriata). Definire e comprendere le dinamiche dell'errore umano risulta pertanto essenziale al fine di ottenere una visione compiuta del concetto di patient safety e del concetto stesso di evento avverso, che in sanità viene generalmente attribuito ai soli comportamenti negligenti o inesperti. In particolare, risulta fondamentale considerare l'errore, componente ineliminabile della realtà umana, come fonte di conoscenza e miglioramento per evitare il ripetersi delle circostanze che hanno portato l'individuo a sbagliare. ${ }^{6}$ Tuttavia, assimilare la Sicurezza del Paziente alla semplice prevenzione dell'errore risulta potenzialmente riduttivo, in quanto non

Corrispondente: Stefano Tardivo, Dipartimento di Diagnostica e Sanità Pubblica, Università di Verona, Strada Le Grazie 8, 37126 Verona, Italia.

Tel.: +39.0458027660.

E-mail: stefano.tardivo@univr.it

Articolo pubblicato secondo la Creative Commons Attribution NonCommercial 4.0 License (CC BY-NC 4.0).

${ }^{\circ}$ Copyright: the Author(s), 2019

Licensee PAGEPress, Italy

QUADERNI - Italian Journal of Medicine 2019; 7(6):7-18 tutti gli errori esitano in un danno ${ }^{7} \mathrm{e}$, soprattutto, identificare errori non equivale a identificarli come cause di danni. ${ }^{8}$ Tali considerazioni evidenziano come il legame tra errore e danno risulti di complessa definizione, al pari della difficoltà di ricondurre più in generale le procedure ai relativi outcome. ${ }^{9}$

A partire dagli anni ' 90 si è assistito ad un cambiamento di paradigma, che ha determinato una migliore comprensione dei molteplici fattori sottesi agli eventi avversi, contribuendo ad una nuova cultura di approccio all'errore. ${ }^{10}$ Le dinamiche alla base dell'errore sono state affrontate in maniera approfondita da molteplici autori; alcuni di essi hanno evidenziato la potenziale rilevanza del cosiddetto fattore umano, ovvero tutti quegli elementi attinenti alla sfera emotiva, cognitiva ed interpersonale in grado di condizionare la performance del soggetto. Nel report To err is human viene richiamata la dinamica dell'errore clinico descritta dallo psicologo James Reason, che definisce gli errori come insuccessi delle sequenze programmate di attività mentali o fisiche nel raggiungere i risultati previsti. ${ }^{7}$ Reason sostiene che le dinamiche dell'errore non sono attribuibili esclusivamente al singolo professionista, ma anche al sistema stesso in cui egli si trova ad operare. Il cuore di tale approccio trova espressione in una sua nota affermazione: gli esseri umani sono fallaci e gli errori si verificano anche nelle migliori organizzazioni. (...) Non possiamo cambiare la condizione umana, ma possiamo cambiare le condizioni in cui gli uomini lavorano. ${ }^{11}$ Anche Leape ${ }^{12}$ concentrò la propria attenzione su strategie che tenevano conto dei limiti e della fallibilità umana, ponendo l'accento sul cambiamento delle condizioni lavorative piuttosto che sull'addestramento. Attraverso l'utilizzo degli strumenti delle scienze cognitive, Reason ${ }^{13}$ si è spinto ad analizzare l'errore dal punto di vista psicologico, riprendendo la classificazione elaborata da Rasmussen ${ }^{14}$ e distinguendo le tipologie di errore in fallimenti nell'esecuzione (skill-based) ed in sbagli (mistake).

Gli errori skill-based sono errori di esecuzione che si verificano quando le azioni pianificate non vengono svolte in maniera adeguata. In particolare, gli slip (sviste) fanno riferimento ad un fallimento dell'attenzione (ad es. volevo prendere il farmaco $X$ ed invece ho preso il farmaco $Y$ ), che si verifica durante lo svolgimento di attività di routine (ossia compiti semplici 
svolti in maniera automatica); mentre i lapse (dimenticanze) corrispondono ad un fallimento della memoria (ad es. il mancato passaggio di consegne a fine turno). Gli errori non commessi durante l'esecuzione pratica dell'azione (mistake) vengono suddivisi in errori rule-based rappresentati dai fallimenti della pianificazione che corrispondono all'applicazione di una regola errata oppure all'applicazione di una regola corretta nella circostanza sbagliata (ad es. applicazione del protocollo per patologia $\mathrm{X}$, ma la diagnosi era $\mathrm{Y}$ ). Infine gli errori rule-based derivano da scelte errate dovute alla mancanza di esperienza o di formazione, alla presenza di informazioni inadeguate oppure all'incapacità di interpretare correttamente le informazioni disponibili mentre gli errori knowledge-based si verificano in situazioni completamente inedite o impreviste, in cui una nuova soluzione deve essere sviluppata ed applicata sul campo (oppure quando tutte le possibilità preesistenti e disponibili si rivelano inefficaci). In questo caso l'individuo che commette l'errore non è a conoscenza di quale sia il comportamento più appropriato.

Vengono identificate inoltre le violazioni, definite come deviazioni intenzionali da parte di un individuo da un protocollo accettato o da uno standard di cura,${ }^{15}$ che non rappresentano veri e propri errori, in quanto riconducibili al concetto di volontarietà dell'azione, ma che possono tuttavia condurre ad eventi avversi di notevole risonanza. Sono state descritte in letteratura quattro tipologie di violazione che comprendono le violazioni di routine, ovvero quelle infrazioni diffuse e frequenti che hanno la potenzialità di diventare parte del modus operandi degli operatori (ad es. in alcuni reparti è prassi accettata che parte della documentazione sanitaria sia incompleta), le violazioni ottimiz$z a n t i$, che nascono dalla volontà di ottimizzare obiettivi personali e spesso non correlati agli aspetti funzionali del compito (ad es. allo scopo di alleviare la noia sul lavoro o di fornire al trasgressore un senso di realizzazione).${ }^{16}$ In questa tipologia di violazione incorre frequentemente il personale trasferito all'interno di un nuovo contesto operativo. Si aggiungono le violazioni situazionali, ovvero quelle violazioni strettamente correlate a difetti di sistema, che si verificano quando una regola non può essere applicata per via delle circostanze (ad es. quando l'attrezzatura richiesta per eseguire una procedura non è disponibile) e le violazioni eccezionali, che si verificano quando la pressione estrema esercitata dalle condizioni ambientali (tempi ristretti, stress, etc.) può impedire al soggetto di attenersi anche alle regole basilari.

Come sottolineato anche da Lawton ${ }^{17} \mathrm{e}$ Ashcroft, ${ }^{18}$ si potrebbe sostenere che la distinzione tra violazioni ed errori non sia fondamentale, essendo la cultura della pratica clinica tradizionalmente focalizzata sugli esiti delle cure anziché sul comportamento dei medici.
Tale tipologia di approccio risulta encomiabile sotto molteplici aspetti, ma può tuttavia rappresentare un rischio per il clinico anche alla luce della attuale giurisprudenza che appare talvolta incoerente nella valutazione della responsabilità. Se ad esempio un medico agendo in maniera contraria al protocollo salvasse la vita ad un paziente, sarebbe improbabile che egli venga sanzionato, mentre se lo stesso medico attenendosi alle linee guida ottenesse un esito negativo per il paziente, egli potrebbe essere ritenuto responsabile per le proprie azioni. Anche la recente adozione di una norma nazionale di riferimento non sembra chiarire esaustivamente tali aspetti. ${ }^{19}$ In tale ottica, un approccio alla sicurezza prioritariamente orientato verso il comportamento dei professionisti rappresenta la chiave di volta per il raggiungimento di una visione allargata del concetto di patient safety.

In letteratura si riscontra una notevole variabilità riguardo l'effettiva incidenza di eventi avversi, dovuta all'eterogeneità delle definizioni e dei metodi di valutazione adottati a livello internazionale. Nonostante la sicurezza del paziente rappresenti un aspetto importante in tutti i setting di cura, la maggior parte degli studi effettuati riguarda il contesto ospedaliero.

Prima di delineare l'entità del fenomeno, occorre necessariamente definire la terminologia di riferimento adottata nell'ambito della sicurezza del paziente. Secondo la definizione fornita dell'OMS, ${ }^{4}$ con il termine Evento correlato alla sicurezza del paziente si intende qualsiasi evento o circostanza che avrebbe potuto determinare, o ha determinato, un danno ingiustificato al paziente. Tale categoria comprende l'Evento avverso (adverse event), ovvero un evento inatteso correlato al processo assistenziale e che comporta un danno al paziente, non intenzionale e indesiderabile. Le possibili forme di danno includono un peggioramento delle condizioni di salute, il prolungamento del ricovero oppure l'esecuzione di procedure diagnostico-terapeutiche aggiuntive. D'altra parte, per evento senza danno (no harm incident) si intende un evento che ha raggiunto il paziente, ma senza determinare conseguenze negative (ad es. caduta di un paziente senza lesioni). Con il termine Evento evitato (near miss) si fa riferimento invece ad un errore che ha la potenzialità di causare un evento avverso (un danno), che non si verifica per caso fortuito o perché intercettato o perché non ha conseguenze avverse per il paziente..$^{20}$ Si pensi ad esempio all'errata marcatura del sito chirurgico o ad un'errata identificazione del paziente intercettate all'ultimo momento. Tali tipologie di evento possono essere indice di gravi malfunzionamenti latenti all'interno del sistema, rappresentando una importante occasione per rendere l'organizzazione più sicura.

Vincent ${ }^{3}$ sottolinea tuttavia come le espressioni near miss e close call (situazioni molto prossime al 
danno) non siano sempre definite con esattezza in letteratura, comprendendo sia gli eventi che non si sono sviluppati fino al punto di danneggiare effettivamente un paziente, sia quelli nei quali un'azione tempestiva ha evitato il disastro.

Alla base di tali definizioni è insito il concetto stesso di prevenibilità dell'evento. Poiché, come già esplicitato, i pazienti possono subire danni anche in assenza di errori medici (ad es. complicanze comuni della chirurgia o effetti collaterali della terapia), in letteratura si tende a distinguere gli eventi avversi prevenibili da quelli non prevenibili. È interessante notare come alcune tipologie di danno, una volta ritenute non prevenibili, siano oggi considerate prevenibili attraverso una gestione più consapevole del rischio (ad es. le infezioni CVC correlate e bundle).

Uno dei primi studi che si propose di indagare l'entità del fenomeno fu l'Harvard Medical Practice Study, che condusse alla revisione di 30.121 cartelle cliniche di pazienti ricoverati presso 51 strutture per acuti nello Stato di New York. ${ }^{21}$ Il tasso di eventi avversi risultò pari al 3,7\%, di cui una proporzione sostanziale $(27,6 \%)$ attribuibile ad una condotta negligente da parte degli operatori sanitari. Relativamente agli esiti, una quota significativa di tali eventi condusse al decesso del paziente $(13,6 \%)$ o alla comparsa di disabilità permanente $(2,6 \%)$. Un passaggio fondamentale fu la pubblicazione del report To Err is Human: Building a Safer Health System da parte del1'Institute of Medicine.? Tale rapporto rappresenta un appello rigoroso, lucido e inconfutabile ad agire per la sicurezza dei pazienti a tutti i livelli del sistema sa- nitario $^{3}$ e può essere considerato come uno dei principali impulsi allo sviluppo della sicurezza del paziente. Il documento riporta un numero considerevole di decessi annuali attribuibili ad errore medico nel contesto ospedaliero (tra i 44.000 e i 98.000 casi), riconoscendo l'errore in sanità come l'ottava causa di morte negli Stati Uniti. Lo stesso ${ }^{22}$ e successivamente altri autori evidenziarono tuttavia come i dati forniti dal report non rispecchiassero adeguatamente l'entità del problema, evidenziando una maggiore incidenza ed innalzando la quota di eventi avversi potenzialmente prevenibili. ${ }^{23}$ Una revisione della letteratura condotta nel 2008 da de Vries et al, comprendente 8 studi scientifici (74.485 pazienti), evidenziò un'incidenza mediana del 9,2\% ed una percentuale di prevenibilità pari al 43,5\%. ${ }^{24}$ Lo studio di prevalenza IBEAS (Iberoamerican study of adverse events) condotto da Aranaz-Andres et al. coinvolse 58 strutture ospedaliere in 5 Paesi dell'America Latina (Argentina, Colombia, Costa Rica, Messico e Perù). ${ }^{25}$ Dall'analisi di 11.379 cartelle cliniche emerse che un numero pari a 1191 pazienti aveva subito almeno un evento avverso associato alle cure. Il tasso di prevalenza puntuale risultò pari al 10,5\% (di cui circa il $60 \%$ prevenibile), associato nel $28 \%$ alla comparsa di disabilità e nel 6\% alla morte del paziente. Ulteriori studi condotti a livello internazionale nel contesto ospedaliero documentano un'incidenza compresa tra il 3,7\% ed il $23,6 \%$ dei ricoveri e suggeriscono un tasso di prevenibilità altamente variabile (Tabella 1$).^{3}$

Makary e Daniel hanno recentemente mostrato come l'errore medico rappresenti attualmente la terza

Tabella 1. Eventi avversi in strutture ospedaliere per acuti in dieci paesi.

\begin{tabular}{lcccc}
\hline Studio & Autori & $\begin{array}{c}\text { Anno di } \\
\text { ricovero }\end{array}$ & $\begin{array}{c}\text { Numero di } \\
\text { ricoveri }\end{array}$ & $\begin{array}{c}\text { Tasso di eventi avversi } \\
\text { (\% di ricoveri) }\end{array}$ \\
\hline Harvard Medical Practice Study (HMPS) & $\begin{array}{c}\text { Brennan } \text { et al., } 1991 \\
\text { Leape } \text { et al., } 1991\end{array}$ & 1984 & 30.195 & 3,7 \\
\hline Utah-Colorado Study Thomas (UTCOS) & Thomas et al., 2000 & 1992 & 14.052 & 2,9 \\
\hline Quality in Australian Health Care Study (QAHCS) & Wilson et al., 1995 & 1992 & 14.179 & 16,6 \\
\hline Regno Unito & Vincent et al., 2001 & 1999 & 1014 & 10,8 \\
\hline Danimarca & Schioler et al., 2001 & 1998 & 1097 & 9,0 \\
\hline Nuova Zelanda & Davis et al., 2002 & 1998 & 6579 & 11,2 \\
\hline Canada & Baker et al., 2004 & 2000 & 3745 & 7,5 \\
\hline Francia & Michel et al., 2007 & 2004 & 8754 & $6,6 *$ \\
\hline Regno Unito & Sari et al., 2007 & 2004 & 1006 & 8,7 \\
\hline Spagna & Aranaz-Andres et al., 2008 & 2005 & 5624 & 8,4 \\
\hline Paesi Bassi & Zegers et al., 2009 & 2006 & 7926 & 5,7 \\
\hline Svezia & Soop et al., 2009 & 2006 & 1967 & 12,3 \\
\hline
\end{tabular}

*Su 1000 giorni di ricovero. Adattato da ‘(2011) Natura ed entità dell'errore e del danno. In: Tartaglia R., Albolino S., Bellandi T. (eds) La sicurezza del paziente. Springer, Milano', con permesso. 
causa di morte negli Stati Uniti (viene calcolato un tasso medio di 251.454 morti all'anno). ${ }^{23}$

In Italia, uno studio retrospettivo finanziato dal Ministero della Salute e realizzato dal Centro Gestione Rischio Clinico della Regione Toscana, che ha coinvolto 5 Aziende Ospedaliere Universitarie (7573 cartelle cliniche), ha evidenziato la presenza di eventi avversi in una percentuale di ricoveri pari al 5,17\% (tasso mediamente inferiore rispetto a quello calcolato in altri studi analoghi a livello internazionale). ${ }^{26}$

Per quanto riguarda il contesto extraospedaliero, uno studio condotto in Francia ha evidenziato come un tasso compreso tra il 3,5\% e il $4,5 \%$ dei ricoveri in area medica e chirurgica fosse legato ad eventi avversi avvenuti al di fuori dell'ospedale, ${ }^{27}$ mentre uno studio osservazionale condotto in Spagna nel contesto dell'assistenza primaria documentò una prevalenza puntuale inferiore all' $1 \%{ }^{28}$

Per quanto concerne l'impatto finanziario associato agli errori medici, le stime indicano che negli Stati Uniti, tra il 2010 e il 2013, grazie alla riduzione dei danni contratti in ospedale, si siano verificati circa 50.000 decessi in meno con un risparmio in termini di spesa sanitaria pari a circa 12 miliardi di dollari. ${ }^{29}$

\section{Dinamiche e tipologie di eventi avversi}

Le tipologie di eventi avversi riscontrabili nella pratica clinica sono estremamente variabili ed in letteratura si registra una sostanziale assenza di modalità univoche di classificazione. Nel report To Err is $\mathrm{Human}^{7}$ viene proposta una rassegna delle principali tipologie di errore associate a danno emerse nel contesto dell'Harvard Medical Practice Study, ${ }^{21}$ distinte in errori diagnostici, di trattamento, preventivi ed altre tipologie di errore. Tra gli errori diagnostici troviamo gli errori o ritardi di diagnosi, la mancata esecuzione degli esami prescritti, la prescrizione di esami o terapie obsoleti ed il mancato intervento sulla base dei risultati dell'esame. Tra quelli di trattamento ritroviamo gli errori nell'esecuzione di procedure, interventi chirurgici ed esami, gli errori di allestimento, somministrazione e monitoraggio delle terapie. Tra gli errori correlati alla prevenzione, la mancata o ritardata somministrazione di terapia profilattica e l'inadeguato monitoraggio dei trattamenti (ad es. per la prevenzione del tromboembolismo venoso). Infine una miscellanea di errori che vanno dai fallimenti comunicativi al malfunzionamento di attrezzature e apparecchiature e a fallimenti di sistema.

La categoria più comune di errori prevenibili è quella degli errori tecnici (44\%), seguiti dagli errori di diagnosi (17\%), relativi alle pratiche preventive $(12 \%)$ e dagli errori correlati alla gestione della terapia $(10 \%)$. Il Framework concettuale proposto dall'OMS ${ }^{4}$ individua 10 macro-categorie tematiche per la Classificazione Internazionale della Sicurezza del Paziente (più avanti specificate), tra cui la tipologia di incidente (13 tipologie, non mutuamente esclusive ad es. qualità della documentazione, ICA, terapia, sangue ed emoderivati ecc.) e gli esiti per il paziente (comprendenti il tipo di danno, la gravità e l'impatto socio-economico). La combinazione dei due fattori consente di raggruppare gli eventi secondo categorie clinicamente significative.

Gli errori correlati alla gestione della terapia hanno storicamente rappresentato un ambito di particolare rilevanza, anche in virtù dell'elevata percentuale di prevenibilità ad essi attribuita in letteratura. ${ }^{30} \mathrm{Si}$ stima che la percentuale di errori correlati alla gestione della terapia farmacologica sia compresa mediamente tra il $12 \%$ ed il $20 \%$ del totale degli errori medici. ${ }^{31}$ Molteplici studi fanno riferimento al Framework fornito dalla National Coordinating Council for Medication Error Reporting e Prevention's Index (NCC MERP Index), che consente di classificare gli errori di terapia secondo una scala di gravità compresa tra il livello minimo $A$ (circostanze o eventi potenzialmente in grado di arrecare danno) ed il livello massimo I (errore che ha determinato o contribuito al decesso del paziente). ${ }^{32}$

Un ulteriore approccio è quello suggerito da $\mathrm{S}$. Buetow e G. Elwyn che nel libro Health Care Errors and Patient Safety, ${ }^{33}$ partendo dall'assunto che buona parte degli errori possa essere riconducibile al comportamento stesso dei pazienti, propongono uno schema riportante numerosi esempi di errori attivi di pianificazione e di esecuzione commessi dal paziente durante le diverse fasi dell' assistenza sanitaria. Come sottolineato dagli autori, il paziente spesso ignora consapevolmente i propri bisogni e le proprie responsabilità nell'ambito del percorso di cura, per scelta oppure a causa di priorità e vincoli concorrenti. I pazienti possono inoltre contribuire ai fallimenti insiti all'interno del sistema, influenzando quei fattori di natura organizzativa che potrebbero indirettamente essere fonte di errore (ad es. la riduzione del livello di vigilanza dovuta all'aspettativa irrealistica che le cure siano prive della possibilità di errore). ${ }^{34}$

\section{Il concetto di errore in sanità}

- La sicurezza del paziente rappresenta una dimensione fondamentale della qualità delle cure.

- Le dinamiche alla base dell'errore umano sono state approfondite da molteplici autori: le tipologie descritte da James Reason comprendono gli errori skill-based (slip e lapse), rule-based e knowledgebased.

- In letteratura si riscontra una notevole variabilità riguardo l'epidemiologia e le modalità di classificazione degli eventi avversi, definiti come eventi inattesi correlati al processo assistenziale che comportano un danno non intenzionale al paziente.

- I pazienti possono contribuire indirettamente alla genesi degli errori in sanità. 


\section{La gestione del rischio clinico: nascita e sviluppo del clinical risk management}

\section{Il concetto di risk management}

Con il termine rischio clinico si definisce la possibilità che un paziente subisca un danno o disagio involontario, imputabile alle cure sanitarie, che causa un prolungamento del periodo di degenza, un peggioramento delle condizioni di salute o la morte. ${ }^{7}$ Il tema dei rischi collegati all'assistenza sanitaria ha radici lontane ed il progresso scientifico e tecnologico hanno contribuito negli ultimi decenni a ridurre l'entità del fenomeno, comportando tuttavia la nascita di nuove tipologie di rischi. Chantler sottolinea a tal proposito come la medicina moderna sia complessa, efficace e potenzialmente pericolosa. ${ }^{35}$

Il concetto di gestione del rischio clinico (Clinical Risk Management) trova una sua codifica autonoma ed originale a partire dagli anni ' 70 , quando negli Stati Uniti si assistette ad un importante aumento della spesa assicurativa dovuto a cause medico-legali collegate alla malpractice. Lo stesso Vincent definì il Risk Management come una risposta organizzativa al bisogno di ridurre gli errori ed i loro costi (...) nel suo senso più ampio il Risk Management include le procedure necessarie a ridurre i rischi in relazione a tutti $i$ fattori di rischio, non semplicemente ai fattori clinici. ${ }^{36}$ In tale prospettiva, anche Kramen et al. identificarono la gestione del rischio come l'insieme delle attività di autoprotezione volte a prevenire le minacce reali o potenziali di perdite finanziarie dovute a incidenti, infortuni o malpractice in ambito sanitario. ${ }^{37}$ L'esperienza maturata nel contesto delle organizzazioni ad alto rischio (ad es. nel campo dell'aeronautica militare e civile o dell'industria nucleare) aveva già dimostrato d'altronde come i rischi potessero essere ridotti attraverso l'implementazione di opportune strategie preventive o correttive. Il contenzioso ha rappresentato indubbiamente un potente impulso per la sicurezza del paziente, ${ }^{3}$ accrescendo la consapevolezza degli eventi avversi, sia nell'opinione pubblica sia tra i professionisti. A tal proposito, è interessante notare come il già citato Harvard Medical Practice Study non fu originariamente avviato per valutare la qualità e la sicurezza delle cure, bensì proprio per stimare il numero di casi potenzialmente indennizzabili nello Stato di New York. ${ }^{38}$ Le prime impostazioni legate a tale approccio risultarono fondamentalmente di tipo punitivo, ed i gravi limiti che ne emersero condussero negli anni successivi ad una profonda revisione teorica del concetto di Risk Management. In particolare, l'approccio sistemico all'errore dimostrò come gli eventi avversi rappresentino il risultato di un gran numero di fattori interconnessi, riconducibili principalmente a difetti di sistema piuttosto che ad errori individuali.
La gestione del rischio clinico corrisponde attualmente allo strumento che mira a migliorare qualità e sicurezza dei sistemi sanitari, attraverso l'identificazione delle situazioni di rischio per i pazienti e la realizzazione di interventi atti a prevenire o controllare tali rischi. ${ }^{4}$ La finalità principale resta quella di concentrare l'attenzione sul miglioramento dei processi e di stimolare lo sviluppo di una cultura condivisa sul tema della sicurezza. In particolare, appare evidente come la gestione del rischio clinico non possa in alcun modo sottrarsi alla logica del miglioramento continuo della qualità, prevedendo un processo ciclico che comprende l'identificazione dei rischi, la valutazione della frequenza e della gravità del rischio (risk assessment), la pianificazione delle attività volte a ridurre o ad eliminare il rischio ed il successivo monitoraggio delle strategie attuate.

Nel recente volume Safer Healthcare: Strategies for the Real World edito da C. Vincent e R. Amalberti, ${ }^{39}$ gli autori delineano cinque possibili strategie per la sicurezza, ciascuna delle quali basata su una specifica serie di interventi: i) la sicurezza come best practice; ii) miglioramento dei processi e dei sistemi in sanità; iii) controllo del rischio; iv) migliorare la capacità di monitoraggio, adattamento e risposta; v) mitigazione dei danni.

Le prime due strategie (sicurezza come best practice e miglioramento dei processi) mirano principalmente ad ottimizzare l'assistenza fornita al paziente, mentre $\mathrm{i}$ restanti approcci (controllo del rischio, monitoraggio, adattamento e risposta e mitigazione) sono maggiormente incentrati sulla gestione del rischio. Ciascun modello presenta limiti e vantaggi peculiari, e comprende specifiche modalità di formazione, apprendimento e miglioramento. All'interno dei setting operativi in cui l'assistenza assume caratteristiche lineari, il controllo dell'esposizione al rischio ed il mantenimento di standard di cura elevati risultano predominanti, a differenza dei contesti più fluidi e dinamici in cui prevalgono il monitoraggio e le tecniche di adattamento (sebbene una solida base procedurale rappresenti in qualunque ambiente un requisito essenziale). Gli autori sottolineano inoltre come molti programmi per la sicurezza si avvalgano inconsapevolmente di diverse strategie e come ciascun setting assistenziale richieda una diversa combinazione delle varie strategie e degli interventi ad esse associati.

Anche il Framework concettuale per la Classificazione della Patient Safety proposto dall'OMS ${ }^{4}$ rappresenta un interessante modello di riferimento per la gestione del rischio clinico. In particolare, vengono definite 10 macrocategorie tematiche relative alla sicurezza del paziente (tipologia d'incidente, esiti sul paziente, caratteristiche del paziente, caratteristiche dell'incidente, fattori contribuenti/rischi, esiti organizzativi, identificazione, fattori di mitigazione, azioni di 
miglioramento, azioni intraprese per ridurre i rischi...), le cui relazioni semantiche sono definite all'interno del modello.

Per quanto concerne gli attori implicati nel risk management, R. Tartaglia e A. Vannucci nel libro Prevenire gli eventi avversi nella pratica clinica sottolineano come nelle organizzazioni sanitarie la gestione del rischio dovrebbe coinvolgere sia il management che gli operatori della prima linea, a partire dall'integrazione della sicurezza dei pazienti negli obiettivi strategici dell'istituzione, fino a diventare parte integrante delle pratiche sanitarie e delle operazioni di supporto tecnico e amministrativo. ${ }^{40}$ Tale considerazione evidenzia un aspetto fondamentale, ovvero l'importanza legata al coinvolgimento trasversale ed integrato di tutti i soggetti operanti all'interno del sistema (compreso il paziente), al fine di accrescere la percezione del ruolo esercitato da ognuno per il miglioramento della sicurezza.

Nel nostro Paese, lo sviluppo e la diffusione di una reale consapevolezza sul tema della gestione del rischio in sanità risale ai primi anni 2000. Il Ministero della Salute, sul versante informativo, ha focalizzato la propria attenzione sulla sorveglianza e sull'analisi degli Eventi Sentinella, ovvero di quegli eventi a soglia zero che non dovrebbero mai occorrere in sanità (ad es. reazione trasfusionale conseguente ad incompatibilità $\mathrm{AB} 0$ ). Per la gestione del rischio clinico risulta fondamentale che all'interno delle strutture sanitarie sia presente la figura del risk manager, concetto ripreso anche dalla Legge 24/2017 (c.d. Legge Gelli-Bianco), che si trova a dover operare nel campo della prevenzione e della gestione degli eventi avversi, avvalendosi di strumenti che gli permettono di conoscere ed analizzare l'errore e di monitorare le azioni di miglioramento attuate.

\section{Gli strumenti di gestione del rischio: principali finalità e risvolti applicativi}

Gli strumenti di valutazione e di analisi di cui si avvale il Risk Management hanno come obiettivo principale quello di andare oltre la semplice identificazione degli atti non sicuri (unsafe acts) del singolo operatore o del team di cura ed individuare i difetti di sistema che si celano dietro tali comportamenti, o che spesso addirittura ne favoriscono la messa in atto.

Alcuni di questi strumenti analizzano gli errori in modo reattivo, cioè studiano a posteriori le condizioni che hanno condotto al verificarsi di un evento avverso; altri analizzano gli errori in modo proattivo, cioè cercano di identificare ed eliminare le criticità del sistema prima che esse contribuiscano alla genesi dell'errore.

Tartaglia e Vannucci affermano a tal riguardo che il livello di approfondimento e di formalizzazione delle analisi può variare in considerazione delle politiche aziendali e delle risorse disponibili e che le modalità di analisi sistemica degli eventi avversi sono sostanzialmente riconducibili a cinque tipologie di attività (Tabella 2)..$^{40}$

I medesimi autori sostengono inoltre che una vera misura del cambiamento culturale e dello sviluppo organizzativo verso la sicurezza dei pazienti risiede proprio nell'effettiva integrazione dell'analisi degli eventi avversi nelle pratiche cliniche e assistenziali.

L'approccio di natura reattiva, in cui l'analisi viene condotta a ritroso rispetto alla sequenza temporale che ha generato l'evento, prevede l'utilizzo di svariati strumenti, tra cui l'incident reporting, la revisione delle cartelle cliniche (ad es. la metodologia global trigger tool, GTT), l'analisi dei dati amministrativi, la review dei processi, la root causes analysis (RCA) e l'audit per gli eventi significativi (significant event audit). Ognuna di queste metodologie viene utilizzata per identificare e analizzare l'errore quando questo già si è verificato 0 era in procinto di verificarsi (nel caso dei near miss).

In particolare, l'obiettivo primario dei sistemi di incident reporting contribuisce alla mappatura del rischio, al fine di predisporre opportune azioni correttive e prevenire il ripetersi degli eventi segnalati. La raccolta dei dati derivanti dai flussi di segnalazione diventa significativa solo se inserita in un processo di analisi e valutazione sistematica, in grado di generare un feedback destinato non solo al segnalatore ma anche a tutti coloro che potrebbero apprendere dall'esperienza. Come sottolineato da Tartaglia e Van-

Tabella 2. Le attività principali per l'analisi degli eventi avversi.

\begin{tabular}{|c|c|}
\hline Attività & Descrizione \\
\hline Identificazione del problema da analizzare & Segnalazione spontanea, revisione cartella clinica, discussione informale di casi clinici \\
\hline Ricostruzione del caso & $\begin{array}{l}\text { Analisi cartella clinica, colloqui con gli operatori e con il paziente/familiari, raccolta procedure, } \\
\text { protocolli, linee guida o letteratura di riferimento }\end{array}$ \\
\hline Analisi del caso & Timeline, checklist o diagramma dei fattori contribuenti, revisione tra pari \\
\hline Report & Descrizione sintetica dell'evento, delle criticità rilevate e delle azioni di miglioramento \\
\hline Follow-up & Condivisione report, selezione e adozione azioni di miglioramento, verifica dei risultati \\
\hline
\end{tabular}


nucci, ${ }^{38}$ la funzione aziendale per la sicurezza dei pazienti deve trasformare una grande massa di dati qualitativi e quantitativi in informazioni utili a valutare il rischio attuale e a produrre conoscenza organizzativa. Nel contesto sanitario francese è stato fortemente promosso il concetto di feedback informativo, attraverso l'istituzione dei Crex (Comité de retour d'expérience), ovvero comitati composti da professionisti sanitari in cui le singole segnalazioni vengono analizzate in maniera approfondita, al fine di favorire la diffusione di conoscenze a livello dell'intera organizzazione. ${ }^{41}$

I limiti riconducibili ai sistemi di segnalazione spontanea risiedono principalmente nei fenomeni di under-reporting (sotto-segnalazione), legati ad una insufficiente motivazione da parte degli operatori (dovuta alla mancanza di feedback) o al timore di azioni disciplinari. In tale prospettiva, Vincent et al. ${ }^{42}$ sottolineano come i sistemi di reporting non possano essere considerati come strumenti di misura affidabili dei livelli di sicurezza, e come i dati da essi derivanti non possano essere utilizzati per la predisposizione di solide analisi prospettiche quantitative.

La root cause analysis (RCA) rappresenta un processo sistematico e completo mediante il quale, a partire dalla segnalazione degli incidenti, vengono individuate le cause che stanno alla radice dell'evento, classificandole in uno schema di riferimento.

I passaggi che definiscono l'analisi delle cause profonde sono: ${ }^{15}$ i) revisione da parte di un team multiprofessionale che conosce i processi coinvolti nell'evento; ii) analisi dei sistemi e dei processi, piuttosto che delle competenze individuali; iii) analisi delle cause profonde, utilizzando domande tipo cosa ... e perché..., fino a quando tutti gli aspetti del processo siano stati rivisti ed i fattori contribuenti individuati; iv) identificazione di potenziali cambiamenti che potrebbero essere apportati ai sistemi od ai processi, per migliorare le prestazioni e ridurre il rischio di eventi avversi o quasi-eventi simili in futuro.

A livello internazionale vengono proposte numerose metodiche di analisi e di rappresentazione delle cause profonde, tra cui l'albero decisionale (incident decision tree) sviluppato dalla National Patient Safety Agency (NPSA), il diagramma di Ishikawa o a lisca di pesce ed il metodo Orion, proveniente dal contesto aeronautico francese. ${ }^{43}$

L'analisi di tipo proattivo, il cui scopo è quello di identificare i problemi in un'ottica preventiva, si basa sull'analisi dei processi che stanno alla base del funzionamento del sistema. I principali strumenti utilizzabili comprendono la FMEA/FMECA, l'albero dei guasti (FTA) ed il Safety Walkaround.

La FMEA/FMECA è una tecnica ampiamente utilizzata in ambito industriale per valutare l'affidabilità di strumenti e processi produttivi. Originariamente, negli anni 40, era applicata alla gestione missilistica in campo militare (poi utilizzata anche dalla NASA). Lo studio dell'errore umano ha agevolato la sua introduzione in ambito sanitario, proprio in ragione del fatto che tale approccio si sposa molto bene con il concetto di errore sistemico. La tecnica FMEA (failure mode effects analysis) è un metodo di valutazione qualitativa basato sull'analisi simultanea delle vulnerabilità, delle relative conseguenze e dei fattori associati ad esse.$^{44} \mathrm{La}$ tecnica FMECA (failure mode effects and criticality analysis) aggiunge una valenza di tipo quantitativo, attraverso la valutazione del livello di criticità dei possibili guasti. L'acronimo HFMEA (healthcare failure mode and effects analysis) si deve invece al Dipartimento degli Affari dei Veterani degli Stati Uniti (Department of Veteran Affairs) in associazione con il National Center for Patient Safety (NCPS). Tali tecniche formalizzano un processo mentale normalmente adottato dai progettisti ed utilizzabile anche per analizzare un processo già in opera. Per poterle applicare è indispensabile che si conoscano fin nei minimi dettagli i processi che si andranno ad analizzare, le interconnessioni tra le varie componenti e le prestazioni ad essi attribuite.

Il Safety Walkaround (SWR), metodologia accreditata a livello internazionale, si realizza attraverso l'effettuazione, da parte di un gruppo dirigenziale, di una visita, percorrendo insieme agli operatori i corridoi e le stanze delle unità operative. ${ }^{45}$ Lo strumento prevede la realizzazione di brevi interviste rivolte agli operatori o ai pazienti, allo scopo di raccogliere le informazioni utili per identificare i rischi attuali e potenziali presenti nella realtà indagata. Le diverse organizzazioni hanno elaborato una propria strategia di SWR, in relazione alla specifica cultura organizzativa ed ai rapporti tra i diversi livelli aziendali. Lo sviluppo di un senso di appartenenza all'istituzione da parte dei partecipanti rappresenta uno dei principali punti di forza legati all'applicazione di tale strumento.

I principali limiti e benefici associati all'utilizzo di diversi strumenti di valutazione del rischio sono riassunti in un articolo di Thomas e Petersen apparso sul Journal of General Internal Medicine nel 2003 (Tabella 3). ${ }^{46}$

Un'ulteriore evoluzione negli strumenti messi a disposizione per valutare l'approccio alla gestione del rischio clinico nelle organizzazioni sanitarie, oltre alle indagini sulla cultura della sicurezza mediante questionari validati (si veda il contributo di Moretti nella presente monografia) ha visto lo sviluppo di strumenti di autovalutazione per rischi specifici (ad es. le infezioni correlate all'assistenza ${ }^{47-49}$ ) o per l'intera organizzazione quali ad esempio per le Aziende e le strutture italiane lo strumento CARMINA (Clinical Assessment of Risk Management: an Integrated Approach $)^{50}$ peraltro già adottato formalmente dalla Regione Friuli Venezia Giulia, Regione Veneto e Liguria. Lo strumento si avvale della definizione di un set di 
standard minimi per la gestione del rischio clinico suddivisi in sette aree di valutazione che ricomprendono la governance, consapevolezza e valutazione, la comunicazione, le conoscenze e abilità, l'ambiente e contesto sicuri, i processi assistenziali, la gestione dell'evento avverso, l'imparare dall'esperienza. Sono stati identificati complessivamente 52 standard suddivisi nelle diverse aree e collegati ad un sistema di pesatura che consentono mediante una rappresentazione grafica a bersaglio di individuare le aree di gestione carente e le priorità di intervento. Si tratta di uno strumento operativo volto a favorire una autovalutazione presso le singole Aziende del livello di gestione dell'area sicurezza del paziente, che consenta loro di collocarsi mediante un sistema di pesatura complessivamente e per le singole aree rispetto ad uno standard e di orientarsi verso l'adozione di buone pratiche per la sicurezza elaborando progetti di innovazione e adattandoli al proprio contesto. Come già evidenziato nel rapporto To err is human infatti la valutazione standardizzata della sicurezza del paziente rappresenta un imperativo cruciale per le organizzazioni nel fornire un adeguato numero di evidenze che documentino le conseguenze avverse (non intenzionali) dell'assistenza medica e sanitaria. ${ }^{\text {? }}$

\section{La gestione del rischio clinico: nascita e sviluppo del clinical risk management}

- L'aumento del contenzioso in ambito sanitario ha determinato una crescente attenzione sul tema della Sicurezza del paziente.

- La gestione del rischio clinico nasce allo scopo di ridurre l'incidenza degli eventi avversi prevenibili e di migliorare la sicurezza dei sistemi sanitari, secondo la logica del miglioramento continuo della qualità.

- Numerosi strumenti contribuiscono alla gestione del rischio clinico, distinguibili principalmente in metodiche di natura reattiva (ad es. root cause analysis) e proattiva (ad es. FMEA/FMECA) ed in strumenti di autovalutazione rispetto agli standard.

\section{Le nuove frontiere della patient safety: verso un approccio integrato e proattivo alla sicurezza delle cure}

La transizione demografica ed epidemiologica, l'evoluzione dei modelli assistenziali e l'innovazione tecnologica in ambito sanitario impongono una continua revisione dell'approccio alla sicurezza del pa-

Tabella 3. Vantaggi e svantaggi dei principali strumenti di analisi e misurazione degli eventi avversi

\begin{tabular}{|c|c|c|}
\hline $\begin{array}{l}\text { Strumenti di analisi e misurazione } \\
\text { degli errori }\end{array}$ & Vantaggi & Svantaggi \\
\hline Morbidity and mortality meetings e autopsie & $\begin{array}{l}\text { - Possono suggerire fattori contribuenti } \\
\text { - Familiare agli operatori sanitari }\end{array}$ & $\begin{array}{l}\text { - Bias retrospettivo } \\
\text { - Bias di segnalazione } \\
\text { - Focalizzato sugli errori diagnostici } \\
\text { - Poco utilizzato }\end{array}$ \\
\hline Analisi delle denunce & $\begin{array}{l}\text { - Fornisce molteplici punti di vista } \\
\text { (pazienti, operatori, legali) }\end{array}$ & $\begin{array}{l}\text { - Bias retrospettivo } \\
\text { - Bias di segnalazione } \\
\text { - Fonti dei dati non standardizzate }\end{array}$ \\
\hline Sistemi di incident reporting & $\begin{array}{l}\text { - Fornisce molteplici punti di vista } \\
\text { nel corso del tempo } \\
\text { - Possono suggerire fattori contribuenti } \\
\text { - Può far parte delle procedure di routine }\end{array}$ & $\begin{array}{l}\text { - Bias di segnalazione degli errori } \\
\text { - Bias retrospettivo }\end{array}$ \\
\hline Analisi dei dati amministrativi & $\begin{array}{l}\text { - Dati facilmente disponibili } \\
\text { - Non costoso }\end{array}$ & $\begin{array}{l}\text { - Può basarsi su dati incompleti e inesatti } \\
\text { - I dati sono disgiunti dal contesto clinico }\end{array}$ \\
\hline $\begin{array}{l}\text { Revisione della documentazione clinica/ } \\
\text { delle cartelle cliniche }\end{array}$ & $\begin{array}{l}\text { - Dati facilmente disponibili } \\
\text { - Di uso comune }\end{array}$ & $\begin{array}{l}\text { - Valutazioni sugli eventi avversi non affidabili } \\
\text { - Costoso } \\
\text { - Documentazione clinica incompleta } \\
\text { - Bias retrospettivo }\end{array}$ \\
\hline \multicolumn{2}{|c|}{$\begin{array}{l}\text { Revisione della documentazione clinica in formato elettronico } \\
\text { toraggio in tempo realeIntegrazione di molteplici fonti di dati } \\
\text { l'inserimento dei datiImplementazione costosa }\end{array}$} & $\begin{array}{l}\text { Non costoso, dopo l'investimento inizialeMoni- } \\
\text { Possibilità di errori nella programmazione e nel- }\end{array}$ \\
\hline Osservazione delle cure prestate al paziente & $\begin{array}{l}\text { - Potenzialmente accurato e preciso } \\
\text { - Fornisce dati non altrimenti reperibili } \\
\text { - Scopre più errori attivi degli altri metodi }\end{array}$ & $\begin{array}{l}\text { - Richiede molto tempo ed è costoso } \\
\text { - Difficoltà di formare osservatori affidabili } \\
\text { - Potenziali problemi di confidenzialità } \\
\text { - Possibile sovrabbondanza di informazioni }\end{array}$ \\
\hline Sorveglianza clinica attiva & $\begin{array}{l}\text { - Potenzialmente accurato e preciso } \\
\text { per gli eventi avversi }\end{array}$ & $\begin{array}{l}\text { - Richiede molto tempo ed è costoso } \\
\text { - Non efficace nell'individuare errori latenti }\end{array}$ \\
\hline
\end{tabular}

Tradotto e adattato da 'Thomas EJ, Petersen LA. Measuring errors and adverse events in health care. J Gen Intern Med. 2003;18(1):61-67', con permesso. 
ziente. Nonostante gli innumerevoli sforzi compiuti al fine di promuovere il passaggio verso una cultura diffusa e creativa della sicurezza permane una vision inadeguata rispetto alle molteplici sfide legate alla patient safety. ${ }^{51}$

Erik Hollnagel ${ }^{52}$ è stato tra i primi ad evidenziare la natura del problema, ponendo l'accento sulla necessità di un cambio di rotta rispetto ad un approccio alla sicurezza che egli definisce come Safety-I, basato su una logica lineare del tipo find and fix (trova ed aggiusta). L'approccio Safety-I presuppone che tutti gli eventi avversi, sia lievi che gravi, abbiano cause individuabili e risolvibili, prevedendo un'ampia gamma di interventi di natura reattiva, quali il rispetto delle politiche e dei vincoli burocratici, la riduzione dei rischi e la creazione di barriere per arginare gli scostamenti dagli standard. L'autore sostiene la necessità di spostare l'attenzione verso un approccio di tipo $S a$ fety-II, ovvero verso una gestione proattiva della sicurezza che si concentra sul come le prestazioni quotidiane solitamente riescano piuttosto che sul perché occasionalmente falliscano. La base di questo nuovo paradigma consiste nel riconoscere come l'assistenza sanitaria sia in larga misura resiliente, e come la sicurezza non possa essere migliorata imponendo un numero crescente di regole. La variabilità e la capacità di adattamento rappresentano un'importante risorsa per l'intero sistema, mentre i tentativi di standardizzare la complessità a livello locale aumentano il divario tra la pratica clinica quotidiana e l'orientamento impositivo offerto dai decisori. La teoria di Hollnagel evidenzia il forte contrasto tuttora esistente tra un approccio reattivo e proattivo alla sicurezza del paziente, sebbene egli non escluda che le due strategie possano risultare complementari.

C. Vincent e R. Amalberti ${ }^{39}$ si spingono ancora oltre, distinguendo tre diversi modelli (contrastanti) di approccio alla sicurezza: i) modello ultra adaptive, fortemente centrato sulla resilienza e sulle capacità di adattamento da parte del personale, tipico dei contesti ad alto rischio e caratterizzati da un elevato grado di expertise (ad es. emergenza-urgenza, trauma center); ii) modello high reliability, in cui l'elevata affidabilità è basata sul lavoro in team e sul rispetto degli standard procedurali (ad es. chirurgia programmata, area intensiva, medicina interna); iii) modello ultra safe, fondato sull'elusione del rischio e sulla supervisione costante, tipico delle aree di cura altamente standardizzate (ad es. radioterapia, medicina trasfusionale).

È interessante notare come i tre modelli rispecchino un diverso grado di instabilità ambientale, e come un approccio intrinsecamente più sicuro possa non risultare applicabile in tutti i setting assistenziali. Ciascuno di tali approcci può ambire a diventare più sicuro mediante il ricorso a strategie diversificate, che prevedano un equilibrio tra $\mathrm{i}$ benefici legati all'adat- tabilità e quelli legati al controllo. In questa prospettiva, Bagnara et al..$^{53}$ si interrogano sulla effettiva possibilità di raggiungimento dello status di High Reliability Organizations (HRO) da parte degli ospedali. Le principali barriere sono rappresentate dalle $l i-$ mitazioni all'efficienza massima, dalla diffusa resistenza ad abbandonare la propria autonomia professionale e da una spiccata tendenza all'autoprotezione individuale da parte degli operatori sanitari. Tali considerazioni impongono una riflessione sul piano della cultura organizzativa, ed in particolare sulla volontà e abilità di un'organizzazione di comprendere e agire per la sicurezza. ${ }^{54}$ Come evidenziato in letteratura, la promozione di una cultura della sicurezza non dovrebbe rappresentare esclusivamente una dichiarazione di intenti, proprio in virtù del profondo grado di influenza che essa esercita sulla volontà di cambiamento. ${ }^{55}$ L'attenzione ai processi sociali e l'instaurazione di un clima di fiducia (in grado di favorire la segnalazione dei rischi) rappresentano elementi chiave per la transizione verso una cultura giusta (just culture), ovvero un sistema di responsabilità condivisa in cui ciascun membro è orientato verso scelte e comportamenti sicuri. Un moderno approccio alla sicurezza non può ignorare i suddetti principi, che vanno ben oltre la mera distinzione tra atteggiamenti reattivi e proattivi e la gestione degli equilibri tra strategie consolidate.

Accanto a queste sfide emergono nuove problematiche, quali il ruolo della Information Technology e la gestione del rischio nel contesto extraospedaliero, che impongono una maggiore attenzione verso una visione integrata della sicurezza ed una partecipazione attiva del paziente (in qualità di partner e co-produttore di cure sicure). Degos et al. ${ }^{56}$ accolgono questa visione più ampia, sottolineando il divario tra gli interventi di sicurezza ospedalieri e le strategie attuate al di fuori dall'ospedale, arrivando a suggerire una nuova categoria di eventi avversi (definiti come eventi avversi integrati), derivanti dalla ripetizione di scelte strategiche errate e da una scarsa organizzazione delle cure.

I quattro pilastri per una strategia della sicurezza proposti dal report Patient Safety 2030, a cura del NIHR Imperial Patient Safety Translational Research Centre, ${ }^{10}$ sembrano rappresentare una proposta coerente rispetto alle numerose sfide emergenti: i) approccio di sistema (l'approccio per ridurre i danni deve essere integrato e implementato a livello di sistema); ii) importanza della safety culture (le organizzazioni sanitarie devono concretamente dare priorità a qualità e sicurezza attraverso una visione stimolante ed un rinforzo positivo e non attraverso il biasimo e atteggiamenti punitivi); iii) i pazienti come veri partner (le organizzazioni sanitarie devono coinvolgere i pazienti e lo staff nella sicurezza in qualità di risorse attive e non semplicemente come vittime o colpevoli); iv) il 
bias rispetto all'azione (gli interventi per la sicurezza devono essere basati su solide evidenze). Tuttavia se queste mancano o devono ancora emergere, gli operatori devono procedere attraverso decisioni prudenti e ragionate piuttosto che ripiegare sull'inazione. $\mathrm{Nel}$ report vengono evidenziate inoltre le potenzialità legate al concetto di collaborazione, anche a livello internazionale, e coordinamento tra tutti gli stakeholder.

Alla luce di tali elementi, si evidenzia l'importanza di proseguire all'interno delle organizzazioni sanitarie nello sviluppo di una visione integrata ed allargata del concetto di patient safety, allo scopo di favorire lo sviluppo di una cultura della sicurezza trasversale, fondata sulla partecipazione e centrata sul paziente.

\section{Le nuove frontiere della patient safety: verso un approccio integrato e proattivo alla sicurezza delle cure}

- L'evoluzione dei sistemi sanitari impone lo sviluppo di un approccio integrato e moderno alla gestione del rischio.

- Alcuni autori evidenziano la necessità del passaggio da una cultura reattiva ad una visione proattiva della sicurezza, attraverso l'adozione di strategie che prevedano un equilibrio tra capacità di adattamento e di controllo del rischio.

- Una visione più ampia della sicurezza deve necessariamente includere la partecipazione attiva del paziente e l'adozione di interventi basati sull'evidenza.

\section{Bibliografia}

1. Institute of Medicine. Crossing the quality chasm: a new health system for the 21 st century. Washington, DC, National Academies Press, 2001.

2. United States National Patient Safety Foundation (2000). Agenda for research and development in patient safety. http:/www.npsf.org/pdf/r/researchagenda.pdf

3. Vincent C. Patient Safety. Second edition. Singapore: Wiley-Blackwell - BMJ Books, 2010.

4. WHO. Conceptual Framework for the International Classification for Patient Safety (v.1.1). Final Technical Report, January 2009.

5. Runciman W, Merry A, Walton M. Safety and ethics in health-care: a guide to getting it right, 1 st ed. Aldershot, UK, Ashgate Publishing Ltd, 2007.

6. Ministero della Salute, Commissione Tecnica sul Rischio Clinico (DM 5 marzo 2003). Risk Management in Sanità - Il problema degli errori. Marzo 2004.

7. Kohn LT, Corrigan JM, Donaldson MS, eds. To err is human: building a safer health system. Washington, DC, Committee on Quality of Health Care in America, Institute of Medicine, National Academies Press, 1999.

8. Hofer TP, Kerr EA, Hayward RA. What is an error? Effective Clinical Practice 2000; 3(6):261-269.

9. Lilford R, Mohammed MA, Spiegelhalter D, Thomson $\mathrm{R}$. Use and misuse of process and outcome data in managing performance of acute medical care: avoiding in- stitutional stigma. Lancet 2004; 363:1147-1154.

10. Yu A, Flott K, Chainani N, Fontana G, Darzi A. Patient Safety 2030. London, UK: NIHR Imperial Patient Safety Translational Research Centre, 2016.

11. Reason JT. Human error: models and management. British Medical Journal 2000; 320:768-770.

12. Leape LL. Error in medicine. Journal of the American Medical Association 1994; 272(23):1851-1857.

13. Reason JT. Human Error, Cambridge, UK: Cambridge University Press, 1990.

14. Rasmussen, Jens. Skills, rules, Knowledge: Signals, Signs, and Symbols and Other Distinctions in Human Performance Models. IEEE Transactions: Systems, Man \& Cybernetics (SMC-13) 1983: 257-267.

15. WHO. Patient safety curriculum guide: multi-professional edition, 2011.

16. Lawton RL. Not working to rule: understanding procedural violations at work. Safety Science 1998; 28: 75-95.

17. Lawton RL, Parker D. Judgements of rule-related behaviour in health care professionals: an experimental study. British Journal of Health Psychology 2002; 7: 253-65.

18. Ashcroft D, Morecroft C, Parker D, Noyce P. Likelihood of reporting adverse events in community pharmacy: an experimental study. Quality and Safety in Health Care 2006; 15: 48-52.

19. Legge 8 marzo 2017, n. 24 (c.d. Legge Gelli-Bianco) "Disposizioni in materia di sicurezza delle cure e della persona assistita, nonché in materia di responsabilità professionale degli esercenti le professioni sanitarie".

20. Ministero della Salute, Dipartimento della Qualità. Sicurezza dei pazienti e gestione del rischio clinico: Manuale per la formazione degli operatori sanitari. Roma: Ministero della Salute, 2006.

21. Brennan TA, Leape LL, Laird N, et al. Incidence of adverse events and negligence in hospitalized patients. Results of Harvard Medical Practice Study I. N Eng J Med 1991; 324: 370-6.

22. Leape LL, Lawthers AG, Brennan TA, Johnson WG. Preventing medical injury. Qual Rev Bull 1993; 19:144-9.

23. Makary MA, Daniel M. Medical error-the third leading cause of death in the US, BMJ 2016; 353:i2139.

24. De Vries EN, Ramrattan MA, Smorenburg SM, Gouma DJ, Boermeester MA. The incidence and nature of inhospital adverse events: a systematic review. Qual Saf Health Care 2008; 17(3):216-223.

25. Aranaz-Andrés JM; Aibar-Remón C; Larizgoitia I et al. Prevalence of adverse events in the hospitals of five Latin American countries: results of the 'Iberoamerican Study of Adverse Events' (IBEAS). BMJ Qual Saf. 2011; 20: 1043-1051.

26. Tartaglia R, Albolino S, Bellandi T et al. Adverse events and preventable consequences: retrospective study in five large Italian hospitals. Epidemiol Prev 2012; 36(34):151-161.

27. Michel M, Quenon JL, Djihoud A, Tricaud-Vialle S, de Sarasqueta AM. French national survey of inpatient adverse events prospectively assessed with ward staff. Qual Safety Health Care 2007;16:369-77.

28. Aranaz-Andrés, Jesús María, et al. "A study of the prevalence of adverse events in primary healthcare in Spain." The European Journal of Public Health 2011; 22 (6): 921-925. 
29. Agency for Healthcare Research and Quality. Partnership for Patients and AHRQ National Scorecard on Rates of Hospital-Acquired Conditions. Rockville, MD: Agency for Healthcare Research and Quality; 2015.

30. Von Laue et al. The epidemiology of preventable adverse drug events. Wien Klin Wochenschr 2003; 115/12: 407-415.

31. Guchelaar H.J. et al Medication Errors: Hospital Pharmacist Perspective. Drugs 2005; 65(13):1735-1746.

32. Wachter RM, Gupta K. eds. Understanding Patient Safety, 3e New York, NY: McGraw-Hill.

33. Buetow S, Elwyn G. Patient safety and patient error. The Lancet 2007; 369:158-161.

34. Hurwitz B, Sheikh A. Health Care Errors and Patient Safety. John Wiley and Sons Ltd, 2009.

35. Chantler $\mathrm{C}$. The role and education of doctors in the delivery of healthcare. The Lancet 1999; 353:1178-1181.

36. Vincent C, Bark P. Accident investigation: discovering why thing go wrong. In: Vincent $C$ (Ed.). Clinical risk management: London: B M J Books; 1995. p. 391-410.

37. Kramen SS, Hamm G. Risk management: Extreme honesty may be the best policy. Ann Intern Med 1999;131963-967.

38. Hiatt HH, Barnes BA, Brennan TA et al. A study of medical injury and medical malpractice: an overview. New Eng J of Med 1989, 321(7):480-484.

39. Vincent C, Amalberti R. Safer Healthcare: Strategies for the Real World. 1st ed. SpringerOpen, 2016, doi:10.1007/978-3-319-25559-0.

40. Tartaglia R, Vannucci A (Eds.). Prevenire gli eventi avversi nella pratica clinica. Springer Verlag, 2013.

41. Lartigau E, Vitoux A, Debouck F. Crex and Orion analysis in radiation oncology: towards a mutualisation of corrective actions. Cancer Radiother. 2009; 13(6-7):458-60.

42. Vincent C, et al. Is health care getting safer? B M J 2008; Vol. 337: 1205-07.

43. Debouck F, Rieger E, Petit H, Noël G, Ravinet L. ORION $\AA$ : a simple and effective method for systemic analysis of clinical events and precursors occurring in hospital practice. Cancer Radiother. 2012 May;16(3): 201-8.

44. Del Grosso P. Metodi di analisi per la gestione del ri- schio. L'analisi proattiva con la FMECA. HPM 2014; 1:37-45.

45. Poletti P. Safety walkaround. Care 2, 2009, 27- 33.

46. Thomas EJ, Petersen LA. Measuring errors and adverse events in health care. Journal of General Internal Medicine 2003 ; 18: 61-7.

47. Cookson B, Mackenzie D, et al. Consensus standards and performance indicators for prevention and control of healthcare-associated infection in Europe. J Hosp Infect 2011; 79: 260-4.

48. Tardivo S, Moretti F, Nobile M et al. Definition of criteria and indicators for the prevention of Healthcare-Associated Infections (HAIs) in hospitals for the purposes of Italian institutional accreditation and performance monitoring. Ann Ig 2017; 29: 529-547.

49. Tardivo S, Moretti F, Agodi A et al. Essential strategies in HAI prevention and control: performance assessment through the implementation of the HAI-CoSIP tool of the GISIO-SItI group. A pilot study in a sample of Italian Organizations. Ann Ig 2018; 30 (Suppl. 2): 70-85.

50. Tricarico P, Tardivo S, Sotgiu G et al. Clinical Assessment of Risk Management: an INtegrated Approach (CARMINA). International Journal of Health Care Quality Assurance 2001, vol. 29, p.744-758.

51. Westrum R. A typology of organisational cultures. Qual Saf Health Care 2004; 13 (Suppl 2): 22-7.

52. Hollnagel E. Safety-I and Safety-II: The Past and Future of Safety Management. Farnham, Surrey: Ashgate Publishing Ltd, 2014.

53. Bagnara S, Parlangeli O, Tartaglia R. Are hospitals becoming high reliability organizations? Applied Ergonomics 2010; 41:713-718.

54. Reiman T, Pietikäinen E, Oedewald P: Multilayered approach to patient safety culture. Qual Saf Health Care 2010; 19(5): e20.

55. Benning A, Ghaleb M, Suokas A et al: Large scale organisational intervention to improve patient safety in four UK hospitals: mixed method evaluation. BMJ 2011; 342: d195.

56. Degos Laurent, Amalberti René, Bacou Jean, Carlet Jean, Bruneau Charles. Breaking the mould in patient safety. BMJ 2009; 338:b2585. 


\title{
Rischio clinico: livelli di staffing infermieristico ed esiti correlati alle cure infermieristiche
}

\author{
Giovanna Pentella, ${ }^{1}$ Letizia Tesei ${ }^{2}$ \\ ${ }^{1}$ Presidente Associazione ANiMO, Ospedale Buonconsiglio Fatebenefratelli, Napoli; ${ }^{2}$ Gruppo Ricerca Associazione ANiMO, \\ Area Vasta 2 ASUR Marche, Ancona, Italia
}

\section{Introduzione}

L'assistenza sanitaria si è evoluta considerevolmente nel corso degli ultimi venti anni. Le conoscenze in merito alle malattie ed alle innovazioni tecnologiche hanno contribuito ad aumentare l'aspettativa di vita nel corso del ventesimo secolo. Ma una delle più grandi sfide oggi non è quella di tenere il passo con le più recenti procedure cliniche o con le più moderne attrezzature altamente tecnologiche, ma di fornire un'assistenza sanitaria più sicura in ambienti complessi, sotto pressione ed in continua evoluzione. In tali ambienti, le cose possono spesso andare male. Gli eventi avversi accadono. I pazienti possono subire danni accidentali, non desiderati ma gravi durante la pratica assistenziale di routine, o come risultato di una decisione clinica. Nei sistemi complessi come la sanità, l'incidente non è quasi mai la conseguenza di una singola azione o decisione, quanto del concatenarsi di una serie di piccoli fattori che in determinate condizioni possono diventare sufficienti, ma non per altro tutti necessari, a produrre l'incidente. Secondo Cook e Woods le attività del management (blunt end) di tipo organizzativo, amministrativo e normativo influenzano e vincolano le risorse cognitive e materiali a disposizione degli operatori sullo sharp end. ${ }^{1,2}$ L'efficacia del sistema dipende pertanto dalla fluidità nell'integrazione tra questi fattori.

Lo scenario degli ultimi decenni dimostra tuttavia una carenza di infermieri in molti paesi ${ }^{3-5} \mathrm{e}$ allo stesso

Corrispondente: Giovanna Pentella, Presidente Associazione ANiMO, Ospedale Buon Consiglio Fatebenefratelli, Napoli, Italia.

E-mail: pentellag@gmail.com

Articolo pubblicato secondo la Creative Commons Attribution NonCommercial 4.0 License (CC BY-NC 4.0).

${ }^{\circ}$ Copyright: the Author(s), 2019

Licensee PAGEPress, Italy

QUADERNI - Italian Journal of Medicine 2019; 7(6):19-24 tempo, evidenzia la presenza di pazienti ospedalizzati gravemente malati, che richiedono tempi e cure intensi. ${ }^{6,7}$

Il Consiglio internazionale degli infermieri (ICN) a tale proposito, ha evidenziato quale sfida comune rivolta ai gestori delle risorse umane il determinare il più efficace mix di personale e competenze necessario per fornire qualità e cure economicamente vantaggiose alla luce della crescente domanda di servizi sanitari, contenimento dei costi e carenza di infermieri e di altri operatori sanitari. ${ }^{8}$

Il fattore umano rappresenta infatti, un elemento primario nei processi di produzione ed erogazione di prestazioni e servizi sanitari in grado di influenzarne il risultato: la sua gestione e valorizzazione, pertanto, assume inevitabilmente valenza strategica per le organizzazioni sanitarie. ${ }^{9}$

La letteratura scientifica dedicata a questo tema ha confermato infatti, quanto i problemi di staffing in sanità, insieme al peso e alla frequenza delle interruzioni del flusso lavorativo siano alcuni dei fattori che impattano maggiormente, talvolta in modo molto grave, sull'organizzazione del lavoro, sulla sicurezza delle cure e sul benessere degli operatori sanitari, compromettendone la performance, aumentando quindi la probabilità di eventi avversi a danno dei pazienti. ${ }^{10}$

Poiché la rilevazione degli esiti rappresenta un elemento strategico per tutti i sistemi sanitari per poter valutare l'efficacia degli interventi e fornire quindi informazioni per migliorare la qualità, la misura degli indicatori sensibili alle cure infermieristiche dovrebbe costituire parte integrante del management sanitario per la valutazione dell'appropriatezza e della qualità degli interventi assistenziali, soprattutto negli ospedali per acuti. ${ }^{11} \mathrm{~A}$ tale proposito, numerosi studiosi hanno cercato di studiare lo staffing infermieristico, gli esiti sensibili alle cure infermieristiche ed oggettivarne la loro relazione in termini di outcome assistenziali e sicurezza delle cure.

\section{Criteri e strumenti di ricerca}

È stata condotta una revisione della letteratura al fine di sintetizzare i principali studi primari e secon- 
dari con disegni diversi tra loro (qualitativi e quantitativi) con l'obiettivo di consentire una comprensione esaustiva del fenomeno indagato. Non è stata prevista tuttavia un'analisi della qualità degli studi inclusi. La ricerca bibliografica è stata condotta consultando attraverso la piattaforma ESBCO le banche dati Medline e Cinahl, il motore di ricerca Google Scholar, la ricerca manuale nelle riviste. La strategia di ricerca non ha imposto limiti temporali di pubblicazioni per il reperimento degli articoli e sono stati utilizzati i seguenti termini, sia per ricerca libera sia utilizzando il MeSh database: Nursing staff, Adverse outcome.

\section{Risultati}

Per staffing infermieristico s'intende il processo di assunzione, posizionamento e supervisione dei dipendenti di un'organizzazione. ${ }^{12}$

In risposta alle preoccupazioni sui livelli di organici infermieristici necessari per garantire sicurezza nelle cure dell'assistito, lo staffing infermieristico è stato attenzionato in letteratura scientifica, sia da un punto di vista quantitativo (rapporto infermiere/paziente) orientando per esempio alcuni paesi (California, Stati Uniti, Australia) a normare rapporti obbligatori tra numero di infermieri e paziente, sia attenzionando lo skill mix infermieristico in termini qualitativi: grado di abilità e livello di formazione rispetto al loro impatto in termini di qualità dell'assistenza e sicurezza nelle cure. Sul problema lo studio di Cho, identifica i due indicatori organizzativi, rapporto tra numero di infermieri e di pazienti con determinazione del nursing workload diretto ed indiretto, e lo skill mix, ovvero l'insieme delle esperienze, conoscenze, capacità e competenze, presenti nel personale dedicato all'assistenza, quali fattori impattanti sugli esiti correlati all'assistenza infermieristica. ${ }^{13}$ Stone e Needelman hanno invece sviscerato $i$ vari aspetti legati alla relazione infermieri-esiti, Stone dimostrando come gli outcome sul paziente possono variare non solo attraverso l'esercizio classico della professione ma anche attraverso la capacità organizzativa e di pensiero critico (critical thinking) da parte dell'infermiere, e Needelman soffermandosi invece, sul carico di lavoro (nursing workload) e sull'organizzazione dell'assistenza, quali elementi necessari a mettere gli infermieri nelle condizioni lavorative ottimali per esercitare al meglio la professione infermieristica. ${ }^{14,15}$

I primi esiti correlati alle caratteristiche dello staffing infermieristico identificati da tali studi sono stati le lesioni da pressione, l'incidenza delle cadute dei pazienti e la customer satisfaction.

Rispetto agli esiti correlati alle cure infermieristiche, anche se il dibattito è recente, alcune teoriche dell'infermieristica avevano già contribuito ad individuarli. Florence Nightingale ad esempio, fu la prima ad ipotizzare anche per l'infermieristica misure di esito tipiche della medicina (mortalità e morbilità), mentre Virginia Henderson dichiarò l'indipendenza del paziente nelle attività di vita quotidiane (ADL) quale esito più importante per valutare l'assistenza infermieristica.

Ma è a partire dalla fine degli anni ' 90 che sono stati istituiti a livello internazionale osservatori sugli esiti correlati alle cure infermieristiche (nursing sensitive outcome) con l'obiettivo di monitorare gli esiti dell'assistenza infermieristica sul paziente, attraverso un set di indicatori prestabilito e quindi misurare la performance assistenziale. Come suggerisce Doran infatti, solo lo sviluppo di database con indicatori di esito in grado di riflettere la qualità dell'assistenza infermieristica ricevuta dai pazienti può rappresentare una preziosa fonte di dati utile per condurre studi e ricerche con possibili ricadute sulle linee politiche e sul1'esercizio della professione. ${ }^{16}$

Allo scopo di superare la carenza di strumenti validati, utili a misurare gli esiti nursing correlati, l'associazione delle Infermiere dello Stato di Alberta (The Alberta Association of Registered Nurses, Canada) ha realizzato nel 1993, una prima revisione delle pubblicazioni disponibili dal 1987 al 1993. Successivamente, nel 1997, l'università dell'Iowa (USA) ha sviluppato il Nursing Outcomes Classification (NOC) che ha guidato il dibattito internazionale per anni sui risultati clinici degli infermieri, mettendo in luce anche i limiti di questa tassonomia, dati dall'eterogeneità dei diversi setting di applicazione, dalla necessità di traduzione $\mathrm{e}$ dalla staticità descrittiva che non consente di valuta $\mathrm{i}$ margini di miglioramento o peggioramento dello stato di salute. Diane Doran e Peter Griffiths sono tra i principali autori che hanno cercato di dare una visione globale della letteratura sugli outcome. Doran definisce i nursing sensitive outcome come la conseguenza o gli effetti degli interventi erogati dagli infermieri e si manifestano con cambiamenti nello stato di salute, nel comportamento o nella percezione del paziente e/o con la risoluzione del problema attuale per il quale l'assistenza infermieristica è stata prestata. ${ }^{16}$ Griffiths descrive i nursing sensitive outcome come aspetti dell'esperienza, del comportamento e dello stato di salute del paziente che sono determinati in tutto o in parte dall'assistenza infermieristica ricevuta, con variazioni che dipendono dalla qualità e dalla quantità dell'assistenza stessa. ${ }^{17}$

Ad oggi gli esiti sensibili alle cure infermieristiche possono essere classificati a seconda dell'ambito al quale si riferiscono in: clinici e di sicurezza, organizzativi e relativi all'ambiente lavorativo, soggettivi dei pazienti e degli operatori. ${ }^{17}$

L'attenzione è stata quindi posta al tema della relazione tra staffing e esiti delle cure infermieristiche, al fine di dimostrare oggettivamente se le caratteristi- 
che dello staff infermieristico piuttosto che il modello organizzativo assistenziale adottato e quindi l'assistenza erogata, rappresentassero fattori determinati gli esiti assistenziali.

Nell'ultimo decennio sono state quindi condotte numerose ricerche che hanno portato ad identificare outcome sensibili alle cure infermieristiche. ${ }^{18}$

La revisione della letteratura di Lancia et al., ha evidenziato a tale proposito come modelli adeguati di assistenza infermieristica possono ridurre alcuni esiti negativi sull'assistiti come la mortalità a 30 giorni, le cadute, gli errori nella somministrazione dei farmaci, le infezioni nosocomiali, le lesioni da pressione, oltre che la durata del ricovero. ${ }^{19}$

Il livello di formazione piuttosto che l'esperienze professionale acquisita dal personale infermieristico incidono sul tasso di mortalità dei pazienti e sugli esiti assistenziali in generale. ${ }^{20}$

La maggior parte degli studi condotti nel setting ospedaliero ha evidenziato la relazione tra organico infermieristico e sicurezza del paziente. ${ }^{21,22}$

A titolo di esempio, gli studi di Aiken e Needleman hanno correlato con dati sperimentali, le cure infermieristiche, alla mortalità e alle complicanze dei pazienti, evidenziando i collegamenti e relazioni negli ambiti della competenza, della prestazione e della sicurezza dei pazienti attraverso la misura di specifici indicatori di esito dell'assistenza erogata. ${ }^{23,24}$

Negli ultimi 5 anni, sul tema sono stati condotti importanti studi internazionali e nazionali. Tra questi, lo studio internazionale E.S.A.Med (Esiti Sensibili dell'Assistenza in Medicina) che ha coinvolto tre Università italiane (Udine, Bologna, Verona) e 10 importanti aziende sanitarie ha avuto l'obiettivo di definire gli esiti assistenziali in base alla quantità di assistenza infermieristica erogata (minuti/persona/die) e allo skill mix assistenziale presente..$^{25}$

Lo studio NSO (Nursing Sensitive Outcome) ha avuto la finalità di creare un osservatorio regionale (Emilia Romagna) sugli esiti di una serie di eventi connessi all'assistenza, come lesioni da pressione le cadute accidentali la contenzione. ${ }^{18}$

In particolare, lo studio RN4CAST condotto a livello europeo e mondiale e che in Italia ha coinvolto 13 regioni, 30 aziende sanitarie e ospedaliere per un totale di circa 3700 infermieri e 3700 pazienti, ha consentito a livello europeo e mondiale di indagare aspetti relativi a: staffing (composizione dello staff infermieristico e rapporto infermieri/persone assistite, cure mancate, la safety/ sicurezza, il burn out, l'ambiente di lavoro, la soddisfazione del paziente e qualità delle cure.

Per quanto riguarda lo staffing (la composizione dello staff infermieristico), è stato evidenziato come ideale per garantire una assistenza infermieristica ottimale un rapporto di linfermiere per 6 assistiti nei reparti ospedalieri e che un aumento di questo rapporto, aggiungendo un paziente ad ogni infermiere ( 1 a 7) aumenti del 6\% la mortalità e del 23\% le cure mancate. In Italia lo staffing rilevato nello studio, evidenzia un rapporto infermiere paziente 1 a 9,54 , con un range variabile dallo 7,08 al massimo di $13,65 .{ }^{26} \mathrm{~A}$ livello internazionale, è stato inoltre dimostrato come una presenza di personale di supporto (in Italia rappresentato dall'OSS) superiore in proporzione al $40 \%$ dello staff infermieristico diventi pericoloso e possa determinare anch'esso un aumento della mortalità. ${ }^{27}$ Altro elemento che incide sugli esiti delle cure assistenziali che emerge dallo studio è la presenza di infermieri laureati nello staff infermieristico. Un incremento del $10 \%$ di infermieri laureati all'interno dello staff corrisponde ad una diminuzione della mortalità pari al $7 \%{ }^{28}$ Rispetto alle cure mancate, le attività che gli infermieri italiani coinvolti nella survey hanno dichiarato di non aver svolto/non essere riusciti a svolgere con maggior frequenza sono state soprattutto attività legate alla sfera della relazione, della comunicazione e dell'educazione, della progettazione e della documentazione delle attività infermieristiche, ma anche quelle legate all'assistenza diretta delle persone, quali ad esempio l'igiene del cavo orale, il cambio posturale e la sorveglianza adeguata del paziente. ${ }^{26}$ Rispetto alla sicurezza ed alla qualità delle cure, l'analisi dei risultati dello studio evidenzia che il $22,9 \%$ degli infermieri che ha partecipato allo studio ritiene che il livello di sicurezza dei pazienti sia scarso e la qualità dell'assistenza bassa $(38,7 \%){ }^{26}$

Sia lo studio di Kalisch et al. che lo studio di Blackman et al. evidenziano che secondo gli infermieri i fattori che causano le cure infermieristiche mancate sono l'allocazione delle risorse non appropriata, la comunicazione tra i professionisti della salute poco efficace, l'intensità del carico di lavoro, la prevedibilità del carico di lavoro, la soddisfazione degli infermieri con il loro lavoro attuale e la loro intenzione di continuare a lavorare. ${ }^{29,30}$

Tuttavia pur se numerosi studi hanno dimostrato l'associazione tra staffing infermieristico e outcome negativi sui pazienti compresa la mortalità, la natura causale di questa relazione è ancora incerta e le mancate cure infermieristiche sono state ipotizzate come fattore che meglio può fornire indicazioni relativamente all'adeguatezza degli standard di personale. ${ }^{31}$

\section{Conclusioni}

Ad oggi numerosi studi dimostrano che bassi livelli di staffing infermieristico sono associati ad una varietà di eventi avversi compresa la mortalità dei pazienti.

I pazienti sviluppano rischi e complicanze, con un aumento della degenza e dei costi: tutti problemi evitabili se fossero assistiti da un numero congruo di infermieri. 
Le direzioni delle Aziende Sanitarie dovrebbero pertanto, investire sulle risorse umane, in termini non solo numerici, ma anche di formazione, di valorizzazione, di competenze. Infatti la qualità e sicurezza dell'assistenza erogata, non sono determinate solo dalla quantità numerica di infermieri, lo staffing level, include i rapporti infermiere/paziente, le ore di assistenza infermieristica erogate (in toto) e le ore erogate dagli infermieri, livelli di competenza, la formazione, lo skill mix cioè la percentuale di presenza di infermieri con qualificazione postlaurea o post diploma rispetto al totale degli infermieri presenti. E' inoltre da prendere in considerazione il burnout, il clima organizzativo, la leadership dei coordinatori e la modalità dell'articolazione della turnazione. In Italia a luglio 2018 sono state diffuse le linee di indirizzo per la predisposizione dei piani dei fabbisogni di personale da parte delle pubbliche amministrazioni dalle quali si evince il passaggio ad una concezione dinamica, basata sulla stesura di piani di fabbisogno del personale triennali e coerenti con il ciclo della performance. Il concetto di fabbisogno di personale viene declinato ora dal punto di vista quantitativo (consistenza numerica) e qualitativo (competenze professionali opportune). La definizione delle risorse umane necessarie si configura esplicitamente, all'interno di queste linee di indirizzo, come un presupposto al perseguimento di obiettivi di performance organizzativa e di erogazione di servizi di qualità. I modelli fondati sulle dotazioni organiche storicizzate, discendenti dalla rilevazione dei carichi di lavoro, sono stati quindi superati ma l'auspicio è che gli studi condotti possano orientate le politiche sanitarie alla definizione del fabbisogno standard necessario per garantire sicurezza e qualità delle cure. ${ }^{32}$

Una delle più grandi sfide odierne è quella di fornire un'assistenza sanitaria sicura in ambienti complessi ed in continua evoluzione. Per questo è necessario determinare il più efficace mix di personale e competenze al fine di fornire qualità e cure sicure. Lo scenario degli ultimi decenni tuttavia, dimostra una carenza di infermieri in molti paesi, a fronte di pazienti complessi che richiedono tempi e cure intensi. La letteratura scientifica conferma quanto lo staffing in sanità, rappresenti uno dei fattori che impatta maggiormente sulla sicurezza delle cure. Numerosi studi hanno cercato di oggettivare la relazione tra caratteristiche dello staff infermieristico, modello organizzativo assistenziale, assistenza erogata quali fattori determinati gli esiti assistenziali. Ad oggi, studi dimostrano che modelli adeguati di assistenza infermieristica riducono esiti negativi quali: mortalità a 30 giorni, cadute, errori nella somministrazione dei farmaci, infezioni nosocomiali, lesioni da pressione, durata del ricovero. Il livello di formazione e l'esperienza professionale del personale infermieristico incidono sul tasso di mortalità e sugli esiti assistenziali in generale. Sul tema sono stati condotti negli ultimi 5 anni impor- tanti studi internazionali e nazionali. Tra questi lo studio internazionale, Registered Nurse Forecasting (RN4CAST) che ha interessato anche l'Italia ha messo in evidenza relazioni tra competenza, prestazione e sicurezza dei pazienti misurando indicatori specifici di outcome. Ad oggi nonostante i risultati evidenziati, solo alcuni paesi hanno affrontato il problema della sicurezza delle cure correlata allo staffing infermieristico introducendo rapporti obbligatori tra numero di infermieri e paziente attenzionando lo skill mix infermieristico qualitativo (abilità e formazione).

\section{Bibliografia}

1. Cook RI, Woods DD. Operating at the sharp end: the complexity of human error. In Bogner, M.S., ed. Human Error in Medicine. Hillsdale, NJ: Lawrence Erlbaum Associates; 1994.

2. Cook RI, Woods DD. "Going solid": a model of system dynamics and consequences for patient safety. Qual Safe Health Care 2005;14:130-4.

3. Buchan J, Calman L. Summary: The Global Shortage of Registered Nurses. International Council of Nurses, Geneva. Geneva: International Council of Nurses; 2005.

4. Buchan J. The nursing workforce and global economic recession: Collegian (Royal College of Nursing, Australia) 2009;16:1-2.

5. Potempa KM, Redman RW, Landstrom G. Human resources in nursing education: a worldwide crisis. Collegian (Royal College of Nursing, Australia) 2009; 16:19-23.

6. Buerhaus P, Needleman J. Policy implications of research on nurse staffing and quality of patient care. Policy. Politics Nurs Pract 2000;1:5-15.

7. Lang T, Hodge M, Olson V, Romano P, et al. Nurse patient ratios: a systematic review on the effects of nurse staffing on patient, nurse employee, and hospital outcomes. JONA 2004;34:326-37.

8. ICN. The Global Nursing Shortage: Priority Areas for Intervention (A Report From ICN/FNIF). International Council of Nurses, Geneva. Geneva. International Council of Nurses; 2006.

9. Turci C, Alvaro R, Fabbretti A, et al. La salute organizzativa degli infermieri in alcune strutture sanitarie di Roma e della provincia. Rivista L'infermiere 2015;6.

10. Hopkinson SG Jennings BM. Interruptions during nurses' work: A state-of-the-science review. Res Nurs Health 2013;36:38-53.

11. Cesa S, Casati M, Galbiati G, et al. Gli esiti sensibili alle cure infermieristiche e i sistemi elettronici per la raccolta di dati clinici: una revisione della letteratura. Rivista L'Infermiere 2014;3.

12. Heneman III J, Judge T. Staffing Organisations. USA: McGraw Hill; 2005.

13. Cho SH. Nurse staffing and adverse patient outcomes: a systems approach. Nurs Outlook 2001;49:78-85.

14. Stone PW. Nursing shortage and Nursing Sensitive Outcomes. Appl Nurs Res 2002;15:115-6.

15. Needleman J, Kutzman E, Kizer K. Performance measurement of nursing care. State of the care and current consensus. Med Care Res Rev 2007;64:10-43. 
16. Doran DM. Nursing outcome: gli esiti sensibili alle cure infermieristiche. Milano: Edizione Mc Graw Hill Education; 2013

17. Griffith P, Jones S, Maben J, Murrells T. State of the art metrics for nursing: A rapid appraisal. London: National Nursing Research Unit, Kings College University of London; 2008.

18. Mongardi M, Bassi E, Caminati A, et al. Regione Emilia-Romagna. Progetto "Osservatorio regionale sui Nursing Sensitive Outcomes"; 2014.

19. Lancia L, Di Labio L, Carpico A, Petrucci C. Aspect and relevant relationship in the nursing workload conceptualization: literature review. Professioni Infermieristiche 2011;64:3-10.

20. Aiken LH, Sloane DM, Bruynell L, et al. RN4CAST consortium. Nurse staffing and education and hospital mortality in nine European countries: a retrospective observational study. Lancet 2014;383:1824-30.

21. Needleman J, Buerhaus P, Pankratz S, et al. Nurse staffing and inpatient hospital mortality. N Engl J Med 2011;364:1037-45.

22. Aiken LH, Sloane DM, Cimiotti JP, et al. Implications of the California nurse staffing mandate for other states. Health Ser Res 2010;45:904-21.

23. Aiken LH, Clarke SP, Sloane DM, et al. Hospital nurse staffing and patient mortality, nurse burnout and dissatisfaction. JAMA 2002;288:1987-9935.

24. Nedleman J, Buerhaus PI, Stewart M, et al. Nurse staffing in hospitals: is there a business case for quality. Health Aff (Milwood) 2006;25:204-11.
25. Palese A, Ambrosi E, Prosperi L, et al. Missed nursing care and predicting factors in the Italian medical care setting. Intern Emerg Med 2015;10:693-702.

26. Sasso L, Bagnasco AM, Zanini M, Catania G. The general results of the RN4CAST survey in Italy general results of the RN4CAST survey in Italy. J Adv Nurs 2017;739:2028-30.

27. Griffiths P, Maruotti A, Recio-Saucedo A, et al. Nurse Staffing, nursing assistants and hospital mortality: retrospective longitudinal cohort study. BMJ Quality Safety 2018.

28. Aiken LH, Sloane DM, Bruyneel L, et al. Nurse staffing and education and hospitality mortality in nine European countries: a retrospective observational study. RN4CAST Consortium. Lancet 2014;383:1824-30.

29. Kalisch BJ, Landstrom GL, Hinshaw AS. Missed nursing care: a concept analysis. J Adv Nurs 2009;65:1509-17.

30. Blackman I, Henderson J, Willis E, et al. Factors influencing why nursing care is missed. J Clin Nurs 2014; 24:47-56

31. Griffiths P, Recio-Saucedo A, Dall'Ora C, et al. The association between nurse staffing and omissions in nursing care: A systematic review. J Adv Nurs 2018;74: 1474-87.

32. Linee di indirizzo per la predisposizione dei piani dei fabbisogni di personale da parte delle pubbliche amministrazioni, Registrato presso la Corte dei Conti il 9 luglio 2018 - Reg.ne - Succ 1477 - Pubblicata in Gazzetta Ufficiale - Serie Generale n. 173 del 27 luglio 2018. 


\title{
Il ruolo delle competenze non tecniche (non technical skill) nella dinamica del rischio clinico
}

\author{
Francesca Moretti, ${ }^{1}$ Michela Rimondini ${ }^{2}$ \\ ${ }^{1}$ Dipartimento di Diagnostica e Sanità Pubblica; e ${ }^{2}$ Dipartimento di Neuroscienze, Biomedicina e Movimento, Università di \\ Verona, Italia
}

\section{Fattore umano e sicurezza del paziente: tra criticità e risorsa}

Il concetto di gestione del rischio trova impulso, prima ancora che in sanità, nel contesto di altri settori ad alto rischio quale l'industria nucleare e aereonautica. L'impatto devastante di eventuali errori in tali ambiti e la loro gestione basata su tecnologie sempre più sofisticate, ha determinato la necessità e la possibilità di garantire livelli sempre maggiori di affidabilità intesa come il grado di fiducia che si può riporre nel sistema, nel suo buon funzionamento e nella sua capacità di assolvere gli obiettivi e le funzioni per le quali è stato progettato. Una organizzazione ad alta affidabilità (HRO, high reliable organization) è in grado di rilevare precocemente, contenere e gestire tutti i rischi intrinseci alle attività che persegue. L'elemento che contraddistingue una HRO non è l'essere priva di errore ma avere la capacità di assorbire tempestivamente l'errore prima che crei una instabilità nel sistema tale da esitare in evento avverso. ${ }^{1}$ Garantire affidabilità significa quindi passare da una concezione della gestione di qualità e sicurezza di tipo reattivo (ossia a posteriori rispetto ad eventuali eventi avversi) a un approccio proattivo (a priori) che permette la creazione, già in fase di progettazione, di metodologie probabilistiche di controllo dei rischi. Ad esempio, l'analisi proattiva dei rischi mediante la FMEA/FMECA (poi adattato nell'ambito sanitario dando sviluppo alla FMEHA e FMECHA), nasce nel contesto aereonautico, con l'obiettivo di svolgere una dettagliata analisi

Corrispondente: Francesca Moretti, Dipartimento di Diagnostica e Sanità Pubblica, Università di Verona, Strada Le Grazie 2 , Verona, Italia.

Tel.: +39.045.807.7294.

E-mail: francesca.moretti@univr.it

Articolo pubblicato secondo la Creative Commons Attribution NonCommercial 4.0 License (CC BY-NC 4.0).

${ }^{\circ}$ Copyright: the Author(s), 2019

Licensee PAGEPress, Italy

QUADERNI - Italian Journal of Medicine 2019; 7(6):25-34 dei potenziali modi di guasto ed errore, studiarne anticipatamente i loro effetti sul comportamento del sistema e comprendere come prevenirli o, quantomeno, minimizzarne occorrenza e impatto.

Tali concetti hanno tardato a svilupparsi in sanità sia perché fino agli anni '90 non era noto il reale impatto dell'errore sulla sicurezza dei pazienti (messo per la prima volta in luce dal famoso report to err is human del $1999^{2}$ ), sia perché il ruolo predominante del fattore umano in tale contesto rende difficile il trasferimento tout court di metodologie progettate per settori a impronta maggiormente meccanicistica. Nell'ambito della gestione del rischio le risorse umane rappresentano infatti sia una fondamentale risorsa che una criticità. La variabilità intrinseca all'operare umano se consente da un lato di conferire al sistema una maggiore resilienza e quindi una maggiore adattabilità a fronte di eventuali deviazioni dall'atteso (errori) che ne potrebbero compromettere il buon funzionamento, dall'altro è potenzialmente essa stessa fonte di errori. Errare è umano racchiude in sé il fondamentale concetto che commettere errori è, di fatto, intrinseco all'operare umano, anche all'operato del più esperto, coscienzioso e tecnicamente preparato operatore. ${ }^{3}$

$\mathrm{Su}$ questo presupposto si fondano i principi dell'approccio sistemico al rischio che identifica negli errori per l'appunto di sistema (ossia fallimenti di tipo organizzativo o di progettazione intrinseci al sistema e quindi perlopiù nascosti) l'obiettivo principale di ogni efficace strategia di gestione del rischio. Sono questi infatti che, nella maggior parte dei casi, creano le condizioni di instabilità che possono portare all'estrinsecarsi dell'evento avverso. In quest'ottica il comportamento dell'operatore (ossia l'eventuale errore attivo compiuto a diretto contatto del paziente), per quanto drammaticamente visibile, non rappresenta nulla di più che l'elemento finale di una catena di fallimenti non evidenti, originatasi molto più a monte. ${ }^{4}$

Come dichiara Reason, uno dei più importanti ricercatori del ruolo del fattore umano nella gestione del rischio: Più che provocare un disastro, gli operatori tendono a ereditare $i$ difetti del sistema [...] il loro ruolo consiste in genere nell'aggiungere il tocco finale 
a una mistura letale $i$ cui ingredienti stanno cuocendo già da lungo tempo [i difetti latenti, di sistema]. ${ }^{5}$

La scienza del fattore umano, ossia quella disciplina che cerca di identificare e gestire i problemi di sicurezza scaturiti dall'interazione tra le persone, l'ambiente di lavoro e le tecnologie ha assunto quindi, in tale contesto, notevole sviluppo. (Human factors engineering is the discipline that attempts to identify and address safety problems that arise due to the interaction between people, technology, and work environments - Clinical Human Factor Group 2015). ${ }^{6}$

Tale disciplina riconosce come imprescindibile la fallibilità e le limitazioni intrinseche all'operare dell'uomo: distrazione, stress e affaticamento, automatismi innescati dalla routine, incomprensioni a livello di comunicazione etc., costituiscono aspetti connaturati all'essere umano $\mathrm{e}$, in quanto tali, predispongono la performance di ogni professionista, indipendentemente dalla sua preparazione, al rischio di commettere errori. Come dice Reason non è possibile cambiare la natura umana ma occorre cambiare le condizioni nelle quali l'uomo lavora. ${ }^{4}$ Obiettivo della scienza del fattore umano è proprio esaminare ogni attività/processo in termini dei diversi compiti che ne determinano il buon esito e quindi valutare se la modalità con cui gli individui operano (fattori individuali), il funzionamento del team, l'organizzazione dell'ambiente di lavoro, il design delle apparecchiature e la tecnologia utilizzata consentono di svolgere i singoli compiti ad un livello ottimale.

Come quindi promuovere sicurezza agendo su tali fattori? In primo luogo, assicurando una adeguata progettazione dei processi e degli ambienti di lavoro (approccio sistemico e gestione dei fattori latenti) che sono il focus primario di qualunque strategia di gestione del rischio. Ma non solo. Se non è possibile cambiare la natura umana è però possibile potenziarne la performance tramite lo sviluppo di alcune abilità definite in letteratura non tecniche (NTS, non technical skills) in quanto attinenti ad aspetti interpersonali e cognitivi (quali la capacità di lavorare in squadra o di prendere decisioni in modo appropriato) che definiscono più il come svolgere un compito piuttosto che il cosa deve essere fatto. ${ }^{6}$

Si discuterà più avanti dei limiti e della ambiguità potenzialmente generata del termine non tecnico che, se ben si addice al contesto della aviazione nel quale trova la sua origine, nel contesto sanitario può essere fonte di fraintendimenti. Ai fini della attuale trattazione appare utile continuare a mantenere la distinzione tra NTS e Abilità Tecniche, così come viene descritta in letteratura. Un esempio emblematico nel contesto della sanità, che chiarisce in parte tale distinzione, sono gli interventi su lato sbagliato che, se pur rari, hanno, quando accadono, conseguenze devastanti. Un'operazione ad un organo sano può essere portata a termine in modo tecnicamente ineccepibile ma costituisce un errore con conseguenze devastanti che trae generalmente origine da fallimenti inerenti all'organizzazione del lavoro (fattori latenti) e da una fatale distrazione o mancanza di coordinamento/comunicazione a livello di team (abilità non tecniche). Porre un adeguato focus sulle NTS permette in ultima analisi di aumentare la resilienza del sistema, in una gestione proattiva dell'errore nella quale l'attenzione alla sicurezza rappresenta parte integrante di ogni attività. In tal senso l'essere umano, in quanto detentore di tali abilità, diventa da elemento di criticità, elemento di forza del sistema.

\section{Resilienza, proattività ed errore: il ruolo delle NTS}

- Un approccio proattivo all'errore è l'elemento chiave di ogni Organizzazione ad Alta Affidabilità (HRO, High Reliability Organization).

- Un adeguato sviluppo delle NTS consente di aumentare la resilienza del sistema, ossia la capacità (propria di una HRO) di assorbire gli errori prima che crei una instabilità nel sistema tale da esitare in evento avverso.

- Potenziare le NTS consente all'essere umano, intrinsecamente predisposto per sua natura ad errori e quindi elemento di criticità del sistema, di diventarne elemento di forza.

\section{Le competenze chiave per la sicurezza del paziente: conoscenze, attitudini e abilità}

Nel paragrafo precedente si è accennato all'importanza assunta nel contesto della gestione del rischio clinico dallo sviluppo delle così dette NTS. Ma quali sono queste abilità e cosa esattamente si intende per non tecniche?

Le NTS vedono il loro iniziale sviluppo nel contesto della aviazione all'interno dei programmi di formazione CRM (crew resource management - gestione delle risorse dell'equipaggio), una delle prime industrie ad alto rischio a focalizzare l'attenzione sul ruolo svolto da tali abilità nella genesi dell'errore e dell'evento avverso. ${ }^{8}$ In seguito, ha trovato applicazione anche in sanità, con l'identificazione di specifiche NTS necessarie all'anestesista (sistema ANTS), al chirurgo (sistema NOTTS) al team di sala operatoria (sistema NOTCHES) e ai ferristi di sala operatoria (sistema SPLINTS). ${ }^{9-12}$

Le NTS sono definite in letteratura come un insieme di abilità cognitive e relazionali sia interpersonali che individuali che integrano le abilità tecniche e contribuiscono allo svolgimento efficiente e sicuro del compito. ${ }^{13}$

Generalmente, vengono incluse tra le NTS ritenute indispensabili indipendentemente dall'ambito di appli- 
cazione, le abilità nel lavoro in team (teamworking), le abilità di leadership e gestione del compito, la comunicazione, la consapevolezza situazionale, le abilità nel prendere decisioni (decision making), la gestione dello stress e della stanchezza. ${ }^{6,14,15}$

Questa lista offre una sintesi delle NTS maggiormente citate, tuttavia non rappresenta un elenco esaustivo, in quanto la definizione di alcune non trova ancora un accordo univoco in letteratura. ${ }^{16}$ Per esempio, uno degli aspetti oggetto di discussione è se abilità comunicative e relazioni attinenti alla interazione con il paziente (es. capacità di ascolto empatico e gestione delle emozioni) dovrebbero rientrare tra le NTS. Tali abilità non sono solo utili ad instaurare una collaborazione e alleanza con il paziente ma si sono dimostrate efficaci nel veicolare cure di elevata qualità e sicurezza. ${ }^{17}$ Inoltre, tali tecniche favoriscono anche il coinvolgimento e l'empowerment del paziente, riconosciuto nel corso degli ultimi decenni come partner essenziale nella gestione e prevenzione del rischio. ${ }^{18,19}$

Capacità quali l'ascolto attivo ed empatico, sono strumenti indispensabili anche nel contesto della relazione tra operatori sanitari, che in genere è il tipo di interazione su cui il filone di ricerca sulle NTS si focalizza. Tuttavia nel suddetto elenco l'accento sugli aspetti di gestione emotiva all'interno del gruppo di lavoro è molto sfumato e identificato genericamente nel supporto reciproco. Questa abilità è in realtà cruciale in svariati contesti di potenziale rischio e interconnessa ad altre NTS. Per esempio, una adeguata gestione dei conflitti è determinata dalla capacità di ascolto e di comprensione reciproca degli interlocutori, non solo sul piano dei contenuti ma anche dei vissuti emotivi. Analogamente, la comunicazione non è solo uno scambio verbale di contenuti (dare e ricevere informazioni), ma è un vero e proprio dialogo influenzato, talvolta anche negativamente, dai vissuti e dalle aspettative dei due interlocutori.

Questa breve digressione sulle diverse possibili abilità che attengono alle NTS apre un'altra questione che merita una riflessione. Sebbene lo sviluppo nel contesto della letteratura delle NTS abbia avuto il merito di spostare l'attenzione sul ruolo fondamentale che tali aspetti svolgono nel contesto della sicurezza, il definirle non tecniche rischia di generare ambiguità e risultare riduttivo rispetto alla elevata preparazione che richiedono. Le NTS vengono infatti generalmente descritte come delle abilità trasversali rispetto all'expertise del singolo professionista attinenti più alla modalità con cui un compito viene svolto, differenziandosi in tal senso dalla abilità tecniche propriamente dette che invece sono specifiche alle aree di lavoro dei singoli professionisti e attengono alle competenze necessarie per l'esecuzione del compito in senso stretto. ${ }^{14} \mathrm{Ad}$ esempio, per eseguire una terapia endovenosa in emergenza si devono possedere una serie di conoscenze su come preparare il farmaco per l'infusione e abilità tecniche su come ottenere l'accesso venoso, come inserire l'ago etc.; d'altra parte è anche essenziale avere una adeguata consapevolezza situazionale, chiarezza nei ruoli e nella gestione delle priorità (lavoro in team e leadership) e adeguate capacità comunicative che, nell'insieme, consentano, nonostante la frenesia propria delle urgenze, di somministrare il farmaco corretto, nella giusta dose, al momento giusto e al paziente giusto (NTS). Similmente anche nel processo comunicativo possiamo individuare questi stessi livelli. Prendiamo ad esempio l'atto del comunicare un possibile elemento di rischio legato ad un trattamento ad un paziente, al fine di renderlo consapevole e coinvolgerlo nella sua prevenzione. L'operatore sanitario dovrà poter far affidamento sulle sue conoscenze (es. percentuale di rischio, fattori di eventuale protezione dal medesimo, ecc.), sulle sue abilità tecniche (utilizzo di tecniche comunicative volte a favorire la comprensione, coinvolgimento e motivazione del paziente es. domande aperte, sintesi, ecc...) e sulle sue abilità non tecniche che permetteranno di adattare lo scambio comunicativo allo specifico paziente rendendolo efficace (es. attitudini dell'operatore e atteggiamento empatico). Come sollevato da alcuni autori, quindi, definire tali aspetti trasversali come non tecnici rischia di generare fraintendimento: tali abilità, a loro volta, si basano sullo sviluppo di adeguate tecniche (ad esempio comunicative, di ascolto, di gestione dei conflitti, etc.) a prescindere dalla predisposizione personale di ciascuno. ${ }^{20,21}$ Lo sviluppo di una comunicazione efficace o di adeguate abilità di lavoro in team richiede pratica costante basata, come si accennerà nell'ultimo paragrafo, sull'uso della simulazione e del feedback.

Alla luce di quanto esposto, un framework di riferimento efficace e completo per identificare al meglio le competenze che ogni professionista dovrebbe sviluppare per garantire cure sicure e di qualità, più che distinguere tra abilità tecniche e non tecniche, dovrebbe basarsi sulla distinzione tra conoscenze, attitudini e abilità. In questa direzione, ad esempio, si è orientato l'Istituto Canadese per la sicurezza del paziente (Canadians Patient Safety Institute, CPSI), una organizzazione no-profit fondata nel 2003 con l'obiettivo di collaborare con decisori politici, organizzazioni sanitarie, leaders e operatori sanitari al fine di promuovere il miglioramento continuo in tema di sicurezza del paziente e qualità delle cure. Tra le attività promosse dal CPSI degno di nota è lo sviluppo di un framework volto a identificare le competenze chiave che dovrebbero diventare parte integrante del bagaglio formativo di ogni professionista impegnato a promuovere pratiche sicure. Il modello proposto dal CPSI identifica 6 domini (cultura della sicurezza, lavoro in team, comunicazione efficace, gestione del rischio, fattore 
umano, gestire gli eventi avversi) e 23 competenze chiave a loro volta basate sullo sviluppo di ben 140 competenze specifiche suddivise in conoscenze, attitudini e abilità. ${ }^{22}$

La Tabella 1 riporta un esempio delle competenze necessarie ai 3 livelli per lo sviluppo di una adeguata comunicazione (Dominio 3).

\section{Lo sviluppo delle NTS: conoscenze, attitudini e abilità}

- Le NTS sono definite in letteratura come un insieme di abilità cognitive e relazionali sia interpersonali che individuali che integrano le abilità tecniche e contribuiscono allo svolgimento efficiente e sicuro del compito (Flin et al., 2010).

- L'implementazione nella pratica clinica delle NTS richiede una formazione continua e si basa, nonostante la definizione di non tecniche, sullo sviluppo di specifiche abilità tecniche.

- Un framework di riferimento efficace e completo per identificare al meglio le competenze che ogni professionista dovrebbe sviluppare per garantire cure sicure e di qualità, più che distinguere tra abilità tecniche e non tecniche dovrebbe basarsi sulla distinzione tra conoscenze, attitudini e abilità.

\section{L'importanza dello sviluppo delle NTS nel contesto della sanità: un focus sulle abilità comunicative e di lavoro in team}

Il contesto sanitario, tra le industrie ad alto rischio, rappresenta dal punto di vista del fattore umano, una di quelle a maggior complessità. Ogni paziente è differente dall'altro e non esiste un modello di essere umano come per i modelli di aerei; non esiste quindi il paziente standard cui fanno riferimento le procedure che, per quanto utili indicazioni, non possono coprire tutte le possibili infinite sfaccettature della complessa gestione e cura di un essere umano. Non sorprende quindi che il ruolo del fattore umano e delle NTS in questo contesto assuma un peso notevole. In accordo con i dati della letteratura si stima che la maggior parte degli errori in sanità, con percentuali fino all' $80 \%$, possano essere attribuiti a NTS inadeguate..$^{23,24}$

In particolare, numerose sono le evidenze che mo- strano una correlazione tra inefficace comunicazione e inadeguato lavoro in team e l'occorrenza di eventi avversi. ${ }^{25-29}$

Per quanto riguarda nello specifico la comunicazione, la Joint Commission evidenzia come errori comunicativi siano alla base di almeno il $70 \%$ degli eventi sentinella e come, fra questi, almeno il $50 \%$ avvenga nella fase di transizione delle cure (passaggio da un setting ad un altro)..$^{30}$

La comunicazione è un fenomeno complesso che richiede per uno sviluppo adeguato il sommarsi di diverse abilità. Una comunemente usata definizione di comunicazione si focalizza sull'aspetto inerente allo scambio di informazioni: Trasmissione di informazioni istruzioni, idee, opinioni che avviene tra almeno due persone, coinvolge competenze verbali e non-verbali ed è tale da essere compreso senza generare confusione. ${ }^{31}$ Nella suddetta definizione viene specificato che il messaggio dev'essere sufficientemente chiaro da non generare problemi nella sua interpretazione. Tale specifica si pone a superamento di un modello lineare di comunicazione per spostarsi verso un modello circolare in grado di tenere in adeguata considerazione il ruolo centrale dei processi di codifica e decodifica e le relative distorsioni che possono nascere tra i due interlocutori. Una efficace comunicazione non dipende infatti solo dal riuscire a trasmettere tutte le informazioni essenziali in modo completo (mancanza di parte delle informazioni) ma anche dal garantire che il messaggio iniziale venga recepito nel modo corretto. Inadeguate comunicazioni da parte dell'inviante, barriere comunicative quali lingue diverse o background culturali differenti, fraintendimenti nella comprensione da parte del ricevente costituiscono potenziali fonti di errori gravi tanto quanto la mancanza di una comunicazione. In realtà, durante una comunicazione, quello che il ricevente comprende non necessariamente coincide con quello che effettivamente era nostra intenzione comunicare. Inviante e ricevente, in altre parole, interpretano il messaggio (codifica e decodifica) ciascuno sulla base delle proprie conoscenze e vissuti (agenda mentale) e l'unico aspetto in grado di correggere eventuali fraintendimenti, incompletezze, errori è il feedback. ${ }^{32}$ Solo una comunicazione contemporaneamente informativa e interattiva (focalizzata sulla ricerca attiva del feedback) è in grado di

Tabella 1. Un esempio di alcune competenze specifiche (conoscenze, abilità e attitudini) necessarie per sviluppare una delle competenze chiave del dominio 3 (Promuovere una comunicazione efficace). (Adattato da CPSI, 2008).

\begin{tabular}{lllc}
\hline Dominio 3 Comunicazione efficace & Conoscenze & Competenze specifiche (esempi) & Abilità \\
\hline $\begin{array}{l}\text { Competenza chiave: } \\
\begin{array}{l}\text { Dimostrare efficaci abilità comunicative } \\
\text { verbali e non verbali }\end{array}\end{array}$ & $\begin{array}{l}\text { Fornire conoscenze sulle comunicazioni strutturate } \\
\text { per promuovere la comprensione reciproca (es. SBAR) }\end{array}$ & Uso di tecniche di & $\begin{array}{l}\text { Mostrare rispetto } \\
\text { ascolto attivo }\end{array}$ \\
\hline
\end{tabular}


cogliere tempestivamente eventuali differenze tra intenzioni (obiettivi) con cui comunichiamo e risultati (quello che viene recepito dal ricevente). La correzione tempestiva di tali differenze è essenziale per garantire efficacia alla comunicazione e prevenire l'estrinsecarsi di errori e eventi avversi. Secondo quanto detto, risulteranno quindi essenziali per una buona comunicazione una serie di strategie volte a comprendere il sistema di codifica altrui (es. ascolto attivo), a verificare la completezza delle informazioni (es. uso di domande aperte), a minimizzare l'effetto distorsivo di vissuti emotivi intensi (es. comunicazione empatica, gestione dei conflitti), a cercare attivamente il feedback (tecniche di chiusura dei cerchi comunicativi) etc... La Figura 1 mostra uno schema delle diverse possibili distorsioni comunicative e di come l'utilizzo del feedback permetta di chiudere il cerchio e di consentire, in tal modo, la verifica della reale efficacia della comunicazione. Chiudere i cerchi comunicativi implica quindi 3 distinte fasi: i) 1'inviante sulla base del suo sistema di codifica (dei suoi vissuti, esperienze, conoscenze, aspettative etc.) invia un messaggio/informazione che può subire ulteriori distorsioni a causa di un canale (ossia dello strumento scelto per la comunicazione) inadeguato (es. uso di comunicazione scritta o fax laddove è preferibile una comunicazione orale) o di disturbi/barriere quali rumore o differenza linguistiche; ii) il destinatario riceve il messaggio e, sulla base del proprio sistema di codifica, lo interpreta (decodifica); e iii) destinatario e/o inviante verificano (mediante l'uso del feedback) che il messaggio sia stato recepito e che non differisca sostanzialmente dal suo contenuto originale.

Un ambito sul quale si è focalizzata molta letteratura nel contesto della comunicazione è il momento del passaggio delle consegne. Il passaggio delle consegne implica il trasferimento e l'accettazione della responsabilità della cura (nel senso ampio del prendersi carico) del paziente. L'Australian Medical Association (2006) definisce il passaggio di consegne come Il trasferimento della responsabilità professionale per alcuni o tutti gli aspetti della cura del paziente (o di un gruppo di pazienti) ad un altro professionista o gruppo di professionisti su base temporanea o permanente. ${ }^{33}$ Questa definizione sottolinea come il passaggio di consegne implichi molto di più di un semplice passaggio di informazioni. Quello che viene passato può essere raffigurato come una sorta di chiave che, teoricamente, permette all'operatore sanitario che riceve le consegne di accedere ad una modalità di gestione del paziente efficace e sicura (con tutte le potenzialmente gravi conseguenze nel caso la chiave non sia quella corretta). Una chiave ben funzionante deve poter garantire l'accesso a diversi aspetti della presa in carico del paziente quali: i) la definizione del piano di azione; ii) la definizione, all'interno del piano di azione, dei ruoli e responsabilità di ciascuno; iii) una adeguata valutazione delle priorità; iv) la capacità di anticipare eventuali problematiche (vision); v) la capacità di gestire tempestivamente eventuali cambiamenti nel quadro clinico (e quindi nel piano di azione) del paziente. Sono questi gli aspetti che, a loro volta, si fanno garanti di elevati outcomes in termini di sicurezza e qualità delle cure. Aspetti che, dall'altro lato non possono prescindere da una comunicazione efficace, ossia una comunicazione che risponda a requisiti di accuratezza, completezza, chiarezza, tempestività, rilevanza e coerenza. Una comunicazione che non risponde a queste caratteristiche, ossia una comunicazione incompleta, poco chiara, non

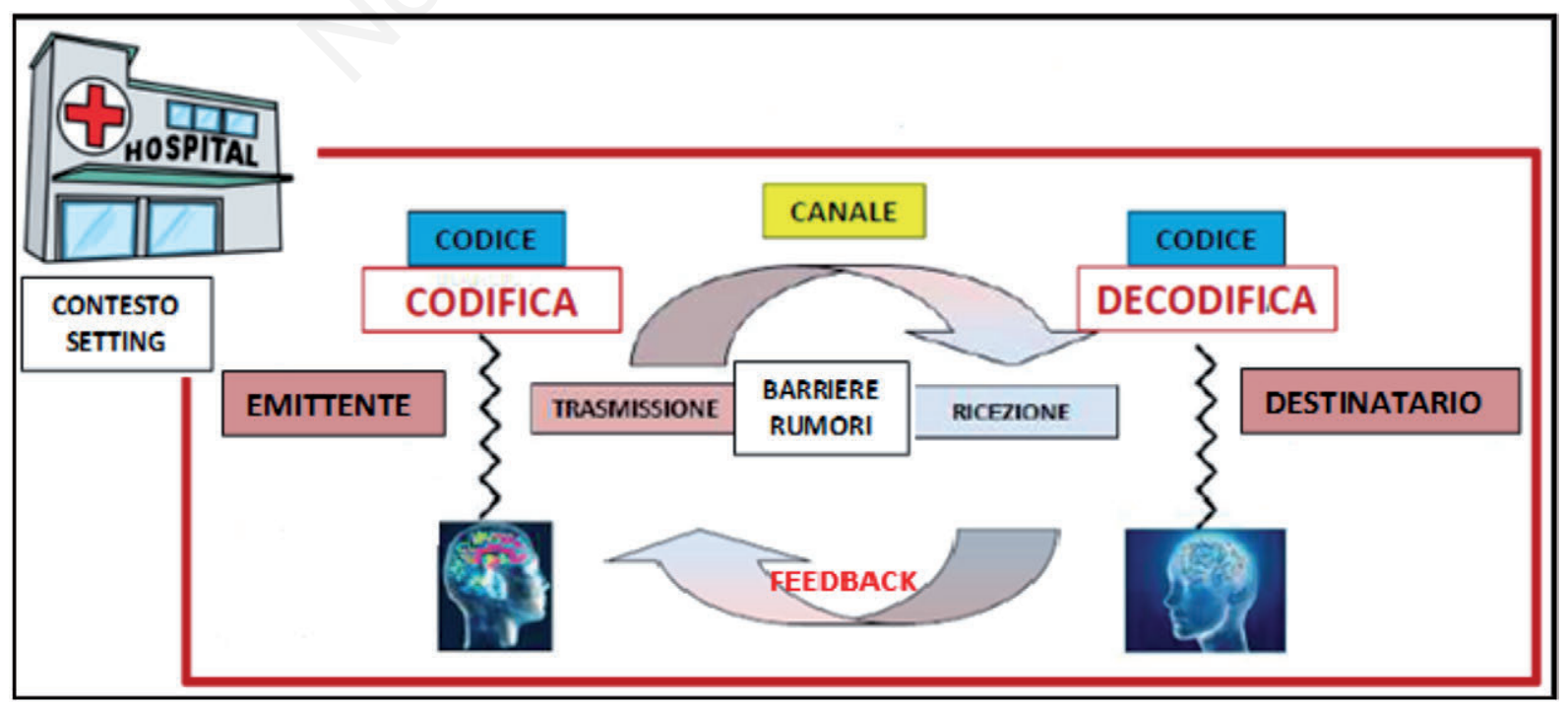

Figura 1. Modello di comunicazione: fonti di distorsioni e ruolo del feedback. 
tempestiva, ridondante e caotica predispone all'estrinsecarsi di eventi avversi. ${ }^{34,35}$

Secondo la letteratura, inoltre, investire su una comunicazione efficace garantirebbe non solo esiti positivi in termini clinici ma anche per quanto attiene un uso più razionale e efficiente delle risorse. Ad esempio, secondo un recente studio condotto in USA gli errori di comunicazione sarebbero, almeno in parte, responsabili, del $30 \%$ di tutte le denunce a carico degli ospedali americani con circa 1744 morti e 1,7 bilioni di dollari spesi in 5 anni. ${ }^{36}$ Inoltre, una indagine su pazienti geriatrici ha riscontrato che nel $54 \%$ dei pazienti rientrati in ospedale entro 28 giorni dalla dimissione vi erano state delle carenze nella comunicazione al momento della dimissione. ${ }^{37}$

La seconda NTS che si è scelto di approfondire nel presente paragrafo, è strettamente legata alla precedente e fa riferimento alla capacità dell'operatore sanitario di lavorare in team. Una definizione ampliamente usata, per quanto datata, individua $\mathrm{i}$ teams come un gruppo distinto di due o più persone che interagiscono dinamicamente, in maniera interdipendente e adattativa al fine di perseguire uno scopo/obiettivo/missione comune, per il quale ad ognuno è stato assegnato un ruolo o una funzione specifica. ${ }^{38}$ Tale definizione richiama l'attenzione su almeno 3 aspetti chiave di un buon lavoro di team, ossia la consapevolezza che i membri sono: i) interdipendenti; ii) orientati ad un unico scopo; iii) complementari nei ruoli. A fronte di ciò un buon coordinamento (sostenuto da una leadership efficace) e uno spiccato senso di cooperazione facilitano la condivisione (di intenti, conoscenze e esperienze) essenziale per il funzionamento in team e per creare quella sinergia di azioni che porta a realizzare risultati superiori rispetto alle performance dei singoli membri che lo costituiscono. In altre parole, i teams, se efficaci creano un valore aggiunto rispetto all'operato dei singoli che va oltre la semplice esecuzione di un compito (taskwork). Ma che cosa identifica il teamwork, ossia, quali sono gli elementi su cui si basa il funzionamento efficace di un team in grado di impattare positivamente sulla sicurezza del paziente?

Risser e colleghi ${ }^{39}$ hanno elaborato un modello che identifica 5 diverse dimensioni proprie di un buon lavoro di squadra, ciascuna delle quali contraddistinta da specifiche caratteristiche (Tabella 2).

Secondo il modello per un buon lavoro di team è innanzitutto essenziale creare un team che sia guidato da un leader e orientato verso specifici e condivisi obiettivi. All'interno del team deve poter vigere un clima di collaborazione, supporto e rispetto reciproco che consenta l'ascolto e la partecipazione di tutti i membri ciascuno con il suo ruolo. Tali aspetti che fanno capo alla prima dimensione del modello gettano le basi per una efficace coordinamento tra membri del team e sono prerequisiti essenziale per il successo delle altre 4 dimensioni. Numerose sono infatti le evidenze che mettono in relazione la sicurezza del paziente con una adeguata leadership e l'instaurarsi di un pattern di coordinamento che vede il team operare come un'unità ben organizzata. ${ }^{29,40,41}$

Tabella 2. La matrice delle dimensioni e i comportamenti di un team efficace.

\begin{tabular}{|c|c|c|}
\hline Dimensioni & Caratteristiche & Comportamenti (esempi) \\
\hline 1. Creare il team e un buon clima di lavoro & $\begin{array}{l}\text { - Stabilire la leadership } \\
\text { - Organizzare il team } \\
\text { - Coltivare un buon clima } \\
\text { - Risolvere i conflitti in maniera costruttiva }\end{array}$ & $\begin{array}{l}\text { - Assegnare i ruoli e le responsabilità } \\
\text { - Tenere in considerazione tutti i membri del team }\end{array}$ \\
\hline 2. Applicare strategie di problem-solving & $\begin{array}{l}\text { - Pianificare strategie di azione adatte } \\
\text { alla situazione } \\
\text { - Applicare strategie di decision-making } \\
\text { - Intraprendere azioni di correzione degli errori }\end{array}$ & $\begin{array}{l}\text { - Identificare procedure e protocolli } \\
\text { - Coinvolgere i membri del team nel } \\
\text { processo decisionale }\end{array}$ \\
\hline 3. Comunicare con il team & $\begin{array}{l}\text { - Utilizzare una comunicazione efficace } \\
\text { - Supportare la raccolta di informazioni } \\
\text { - Supportare la richiesta di informazioni }\end{array}$ & $\begin{array}{l}\text { - Utilizzare il feedback per verificare la correttezza } \\
\text { delle informazioni } \\
\text { - Comunicare il piano di azione agli altri membri } \\
\text { del team }\end{array}$ \\
\hline $\begin{array}{l}\text { 4. Mettere in atto le strategie di azione } \\
\text { e gestire il carico di lavoro }\end{array}$ & $\begin{array}{l}\text { - Implementare i piani di azione } \\
\text { - Triage } \\
\text { - Stabilire le priorità } \\
\text { - Gestire le risorse del team e il carico } \\
\text { di lavoro } \\
\text { - Controllo reciproco delle azioni da parte } \\
\text { dei membri del team } \\
\text { - Mantenere una buona situation awareness }\end{array}$ & $\begin{array}{l}\text { - Gestire le situazioni che si discostano dal } \\
\text { protocollo o dalle decisioni del team } \\
\text { - Stabilire le priorità per ciascun paziente } \\
\text { - Monitoraggio }\end{array}$ \\
\hline 5. Migliorare le abilità del team & $\begin{array}{l}\text { - Implementare strategie per il miglioramento } \\
\text { del team (sia formali che informali) }\end{array}$ & $\begin{array}{l}\text { - Meeting per la discussione di alcuni casi } \\
\text { - Training formativi }\end{array}$ \\
\hline
\end{tabular}

Adattato da Risser et al., $1999^{39}$, con permesso. 
Le dimensioni 2, 3 e 4 rappresentano gli obiettivi/compiti che quotidianamente un team deve assolvere. Gli autori sottolineano come tali aspetti si ripresentino più volte in maniera intercorrelata all'interno di uno stesso turno di lavoro inducendo i membri del team a dover spostare continuamente il loro focus dal lavoro proprio della clinica quotidiana ad aspetti organizzativi sovra individuali. Per un buon lavoro di team è quindi innanzitutto essenziale poter condividere il modello mentale che guida all'azione. A tal fine tutte le informazioni che provengono dall'ambiente devono (schematicamente) passare attraverso 3 momenti fondamentali sostenuti da diverse NTS: i) Capacità di aggiornare costantemente le informazioni che provengono dall'ambiente (es. monitoraggio clinico del paziente) al fine di avere una chiara mappa mentale di quanto stia succedendo. Buone capacità di monitoraggio e consapevolezza situazionale sono fondamentali per non perdere aspetti importanti che comporterebbero una pericolosa errata interpretazione della realtà; ii) Le informazioni devono quindi essere integrate e condivise tra i membri del team. Solo una buona comunicazione può sostenere tale processo che ha come risultato principale la costituzione del modello mentale condiviso, ossia quella capacità da parte dei membri di un team che lavorano in modo coordinato, cooperativo e sinergico di essere tutti sulla stessa pagina rispetto alla realtà clinica che deve essere gestita (condividere chiarezza su obiettivi, ruoli e responsabilità di ciascuno). A tal fine fondamentale è integrare nella pratica quotidiana modelli strutturati di comunicazione (quali il briefing/debriefing o l'uso di framework per il passaggio di consegne come la $S B A R$ ) che diventano preziose occasioni di confronto e integrazione; iii) A fronte del modello mentale condiviso così costituito, i membri del team devono quindi poter scegliere un adeguato piano di azione (fase decisionale), ossia il percorso terapeutico/assistenziale che meglio si adatta a ciascun paziente (Dimensione 2).

Saper comunicare con gli altri membri del team e condividere tutte le idee più rilevanti in maniera chiara e esplicita è quindi propedeutico ad una comprensione condivisa del problema e per poter conseguentemente attuare una efficace collaborazione tra i vari membri e operare in maniera coordinata (Dimensione 3 ).

Un altro modello diffuso in letteratura è quello proposto da Salas e colleghi. ${ }^{38}$ Il framework si basa su 5 dimensioni che identificano a loro volta 5 componenti di un team efficace, ossia, leadership, monitoraggio reciproco, supporto reciproco, adattabilità (resilienza) e attitudine al lavoro in team.

La Tabella 3 riassume brevemente le 5 dimensioni e le reciproche interrelazioni.

All'interno del modello Salas sottolinea inoltre l'importanza di una comunicazione efficace fondata sul chiudere i cerchi comunicativi.
Inoltre, il supporto reciproco su cui si fonda un team efficace e la sua conseguente adattabilità, tutela i membri del team da un eccessivo sovraccarico di stress e/o stanchezza che le evidenze correlano a maggior rischio di errori e eventi avversi. ${ }^{42,43}$

Gli elementi proposti da Salas non si discostano significativamente nei contenuti rispetto a quelli proposti da Risser. Tuttavia, il framework offre il vantaggio di dare una prospettiva dinamica di come le varie componenti del teamwork si condizionino reciprocamente e di come agire in team sia molto di più che sviluppare una lista definita di abilità.

Per concludere, un cenno a parte merita il problema della gerarchia. Le evidenze identificano in una organizzazione gerarchica (di cui ancora soffre il contesto sanitario e in particolare quello italiano) una delle principali barriere al buon funzionamento di un team. Una gerarchia rigida non solo impedisce quel clima di condivisione e collaborazione alla base dei modelli di teamwork efficace presentati ma porta anche a nascondere invece che gestire eventuali conflitti con ulteriori potenziali ripercussioni negative sulla sicurezza dei pazienti. ${ }^{44}$

\section{Capacità di lavoro in team e comunicazione} efficace: elementi chiave per la sicurezza delle cure

- Fallimenti comunicativi e inadeguate capacità di lavoro in team condizionano fortemente la qualità e sicurezza delle cure erogate al paziente oltre che ostacolare un uso efficiente delle risorse.

- Tra gli aspetti chiave di una comunicazione efficace e sicura un ruolo centrale è svolto dall'implementazione del feedback (chiudere $i$ cerchi comunicativi) che consente la correzione tempestiva di eventuali distorsioni comunicative.

- Le dimensioni chiave di un team efficace si basano sulle capacità di lavorare in modo coordinato e sinergico (leadership), di condividere lo stesso modello mentale e adattarlo tempestivamente al mutare delle condizioni (consapevolezza situazionale), di supportarsi reciprocamente mostrando resilienza rispetto a condizioni di sovraccarico (gestione dello stress e della fatica).

- Lavorare per appiattire le gerarchia contribuisce a creare un terreno favorevole allo sviluppo di una comunicazione aperta anche rispetto a tematiche inerenti la sicurezza.

\section{Il ruolo del training nello sviluppo delle NTS}

A conclusione del capitolo, un breve cenno merita l'importanza della formazione nel promuovere lo sviluppo di adeguate NTS in grado di garantire l'erogazione di cure sempre più sicure.

Da quanto premesso è possibile evincere che una comunicazione interprofessionale disfunzionale incide 
negativamente sul clima lavorativo e sul presidio della sicurezza della presa in carico innalzando così il rischio del verificarsi di eventi avversi o errori involontari.

Le aziende sanitarie dovrebbero pertanto porre un accento più marcato e deciso nel valorizzare le occasioni di interscambio tra operatori in una triplice modalità. In primis, considerando l'opportunità di adottare tra le misure volte a selezionare il personale da inserire in attività assistenziale, strumenti che valutino il possesso delle minime attitudini e abilità relazionali predisponenti a un soddisfacente lavoro di equipe e di interazione con il paziente. Questo ampliamento dei criteri di selezione nella formazione e nella assunzione di personale, è nel campo dell'aeronautica da tempo adottato con successo nel reclutamento dei piloti. Se fino ad alcuni anni fa il parametro principale erano le abilità di volo, attualmente accanto a tali prerequisiti si è inserito anche il possesso di capacità definite non technical skills efficaci nel prevenire e gestire le situazioni critiche. ${ }^{45}$

Un secondo livello di valorizzazione risiede nella formazione, che dovrebbe tendere allo sviluppo delle suddette abilità con corsi e supervisioni mirate. Esempi di tematiche che dovrebbero essere oggetto di imple- mentazione di corsi ad hoc sono: la comunicazione assertiva, la gestione del conflitto, la negoziazione e condivisione di un accordo, il problem solving, il team building, ecc... Strumenti come la SBAR o la checklist di sala operatoria, se preceduti da una adeguata formazione, si dimostrano efficaci nel sostenere lo sviluppo di una comunicazione e di un lavoro di team efficace. ${ }^{46,47}$ Analoghe prove di efficacia riguardano percorsi di formazione orientati allo sviluppo di NTS nel contesto sanitario ma rappresentano esempi isolati più che percorsi sistematici di formazione continua. ${ }^{48}$

Infine, la vision di un'azienda sanitaria orientata alla tutela e promozione della comunicazione efficace, assume reale credibilità e concretezza solo se trova riscontro nella regolamentazione e organizzazione della pratica clinica. Il processo comunicativo, infatti non richiede solo delle competenze (intrinseche o acquisite), ma anche spazi e tempi idonei al suo attuarsi. Prendendo ad esempio una delle più consolidate attività di interscambio comunicativo, ovvero il passaggio di consegne tra operatori nel cambio di turno, è evidente che la valenza e utilità che essa assume varierà a seconda della modalità in cui essa avrà luogo. A seconda dell'estrinsecarsi di una serie di fattori organiz-

Tabella 3. Le dimensioni del modello di Salas (2005).

\begin{tabular}{|c|c|c|}
\hline Dimensione & Scopi & Interrelazione con le altre dimensioni \\
\hline Team leadeship & $\begin{array}{l}\text { Sostiene il coordinamento e la cooperazione dei membri del team e ne } \\
\text { garantisce il funzionamento come una unità che agisce in modo sinergico. } \\
\text { In particolare, favorisce l'instaurarsi di processi di soluzione dei problemi } \\
\text { basati sulla condivisione tra membri del team degli obiettivi } \\
\text { (modello mentale condiviso) sul coordinamento di ruoli interdipendenti } \\
\text { e sul sostenere la motivazione di tutti i membri }\end{array}$ & $\begin{array}{l}\text { Il team leader influenza positivamente } \\
\text { l'efficacia di un team tramite la promozione } \\
\text { di comportamenti di monitoraggio } \\
\text { e supporto reciproco }\end{array}$ \\
\hline
\end{tabular}

Monitoraggio reciproco della performance

Nei team efficaci ciascun membro ha consapevolezza di come sta operando il team stesso: ciascun membro è chiamato a monitorare (consapevolezza e monitoraggio situazionale) l'operato altrui al fine $\mathrm{di}$ captare eventuali errori o distrazioni prima che possano raggiungere il paziente e causare effetti avversi
Il monitoraggio reciproco della performance opera attraverso comportamenti di support reciproco e è possibile solo in teams in grado di condividere lo stesso modello mentale e un clima di fiducia

Supporto reciproco Consiste nel supportare un altro membro del team con le adeguate risorse I comportamenti di support influenzano quando si riconosce un problema di inadeguata distribuzione e assegnazione del carico e delle responsabilità di lavoro. Sono 3 le principali modalità di supporto: i) fornire adeguato feedback o supervisione finalizzati al miglioramento della performance;

iii) completare il compito assegnato ad un altro membro del team se si rileva, nei confronti di quest'ultimo, un sovraccarico di lavoro

direttamente l'efficacia di un team garantendo che vengano portati a termine tutti i compiti che gli sono assegnati. Agisce inoltre potenziando l'adattabilità di un team (resilienza) ossia la sua capacità di rispondere efficacemente a cambiamenti sia interni che esterni ii) assistere un membro del team nell'esecuzione di uno specifico compito;

Necessita e si fonda su un adeguato modello mentale condiviso e sul monitoraggio reciproco

\begin{tabular}{ll}
\hline Adattabilità & Corrisponde alla abilità di riconoscere deviazioni rispetto a quanto \\
(resilienza) & atteso (pianificato) e riadattare tempestivamente il piano di azione
\end{tabular}

Attitudine al lavoro È una attitudine a promuovere la performance individuale attraverso la in team coordinazione con gli altri membri del team e la capacità di utilizzarne e valutarne suggerimenti/input finalizzati a portare a termine l'obiettivo condiviso
Ha un effetto diretto sulla efficacia di un team e si fonda su un adeguato modello mentale condiviso e su un efficace monitoraggio e supporto reciproco

Si fonda sul monitoraggio e sul supporto reciproco e sulla predisposizione ad accettare feedback e/o assistenza da parte di altri membri del team

Adattato da Salas et al., $2005^{38}$, con permesso. 
zativi e gestionali (atteggiamento del coordinatore, tempo dedicato all'attività, carico di lavoro clinico routinario, ecc...) tale momento potrà essere vissuto come una afinalistica, stereotipata, congestionata formalità da espletare nei pochi minuti che precedono la fine del proprio orario di servizio - o viceversa come una reale occasione di debriefing in cui le criticità evidenziatesi durante il proprio turno di lavoro fungono da strumento per costruire le priorità dei colleghi entranti in attività.

\section{NTS e formazione}

- Le evidenze inerenti il ruolo delle NTS nel promuovere un assistenza sicura e di qualità responsabilizzano $\mathrm{i}$ decisori in campo sanitario a valorizzare e promuovere la formazione su tali competenze.

- Una rivalutazione dei criteri di selezione del personale (che tenga conto anche di attitudini e abilità relazionali e comunicative) così come un potenziamento delle attività formative inerenti le NTS, rappresentano aspetti imprescindibili per lo sviluppo di organizzazioni ad alta affidabilità (HRO).

\section{Conclusioni}

È indubbio che le abilità comunemente incluse nelle NTS dovrebbero essere insegnate già nelle fasi precoci del training degli operatori sanitari e diventarne parte integrante. Ridurre il training sulle NTS a corsi isolati (per quanto complessi e corredati da simulazione, supervisione e formazione su campo) effettuati nel post-laurea implica lo sminuire la grande preparazione che richiede il loro sviluppo. Comunicazione, abilità di team e le altre NTS di cui si è solo accennato, in virtù del loro notevole impatto sulla sicurezza del paziente e la qualità delle cure erogate dovrebbero invece diventare oggetto di formazione continua a partire già dagli studenti in fase di tirocinio.

Un tale investimento renderebbe possibile non solo un potenziamento del ruolo del fattore umano nella gestione del rischio clinico, ma anche la diffusione di una adeguata cultura della sicurezza, elemento indispensabile per orientare le attitudini dei professionisti sanitari a vivere le pratiche relative alla sicurezza non come un valore aggiunto ma come una responsabilità che attiene trasversalmente la pratica clinica di ognuno.

\section{Bibliografia}

1. Sutcliffe KM, Paine L, Pronovost PJ (2017). Re-examining high reliability: actively organising for safety. BMJ Qual Saf;26:248-251.

2. Institute of Medicine (US) Committee on Quality of Health Care in America. To Err is Human. Building a
Safer Health System. Editors: Linda T. Kohn, Janet M. Corrigan, and Molla S. Donaldson. Washington (DC): National Academies Press (US); 2000.

3. Reason J (1995). A systems approach to organizational error, Ergonomics;38(8):1708-1721.

4. Reason J (2000). Human error: models and management. BMJ;320(7237):768-770.

5. Reason J: Human Error Cambridge: Cambridge University Press; 1990.

6. Ives $\mathrm{C}$, Hillier $\mathrm{S}$, for the Clinical Human Factor Group (CHFG) (2015). Human factors in healthcare: common terms. Disponibile al sito: https://chfg.org/wpcontent/uploads/2016/03/chfg-human-factors-commonterms.pdf (accesso Gennaio 2019).

7. AHRQ. Patient Safety Network. Human Factors Engineering. Last update January 2019. Disponibile al sito: https://psnet.ahrq.gov/primers/primer/20 (accesso Gennaio 2019).

8. Kanki BG, Helmreich RL, Anca Jr. JM: Crew resource management. 2nd ed. ed: Academic Press, 2010.

9. Fletcher G, Flin R, McGeorge P, Glavin R, Maran N, Patey R (2003). Anaesthetists' non-technical skills (ANTS): evaluation of a behavioural marker system. British Journal of Anaesthesia;90(5):580-8.

10. Yule S, Flin R, Paterson-Brown S, Maran N, Rowley D (2006). Development of a rating system for surgeons' non-technical skills. Med Educ;40: 1098-1104.

11. Sevdalis N, Davis R, Koutanji M, Undre S, Darzi A, Vincent C. Reliability of a revised NOTECHS scale for use in surgical teams. Am J Surg 2008;196:184-190.

12. Mitchell, L., Flin, R., Yule, S., Mitchell, J., Coutts, K. \& Youngson, G. (2012). Evaluation of the Scrub Practitioners' List of Intraoperative Non-Technical Skills (SPLINTS) system. International Journal of Nursing Studies;49:201-211.

13. Flin R, Patey R, Glavin R et al. (2010) Anaesthetists' nontechnical skills. Br J Anaesth 105, 38-44.

14. Kodate N, Ross AJ, Anderson JE, Flin R (2012). NonTechnical Skills (NTS) for Enhancing Patient Safety: Achievements and Future Directions. Working Papers 201227, Geary Institute, University College Dublin. Disponibile al sito: https://ideas.repec.org/p/ucd/wpaper/ 201227.html (accesso Dicembre 2018).

15. Engle N, Patey RE, Ross S, Wisely L (2008). Nontechnical skills. Student BMJ 16:454-445.

16. Gordon, Morris, Baker, Paul, Darbyshire, Daniel, Catchpole, Ken and Schocken, Dawn (2014). Devising a consensus definition and framework for nontechnical skills in healthcare to support educational design: A modified Delphi study. Medical Teacher;37(6):572-577.

17. Ross AJ, Anderson JE, Kodate N, et alSimulation training for improving the quality of care for older people: an independent evaluation of an innovative programme for inter-professional educationBMJ Qual Saf 2013;22: 495-505.

18. World Health Organisation: World Alliance for Patient Safety. Forward Programme 2005. Geneva: World Health Organisation (2004). Disponibile al sito: http://www.who.int/patientsafety/en/brochure_final.pdf (Accesso Gennaio 2019).

19. Consiglio Europeo [European Council]: Raccomandazione del consiglio del 9 giugno 2009 sulla sicurezza dei pazienati, comprese la prevenzione e il controllo delle 
infezioni associate all'assistenza sanitaria. Gazetta ufficiale dell' Unione europea (2009/C151/01). Disponibile al sito: https://ec.europa.eu/health//sites/health/files/patient_safety /docs/council_2009_it.pdf (accesso Gennaio 2019).

20. Nestel D, Walker K, Simon R, Aggarwal R, Andreatta P (2011). Nontechnical skills: an inaccurate and unhelpful descriptor? Simulation in Healthcare: The Journal of the Society for Simulation in Healthcare 6(1):2-3.

21. Kardong-Edgren S. (2012). Non-Technical Skills vs Therapeutic Use of Self: An Observation. Clinical Simulation in Nursing; 8:e35.

22. Canadian Patient Safety Institute (2008). The safety competencies: enhancing patient safety across the health professions. Disponibile al sito: http://www.patientsafetyinstitute.ca/English/toolsResources/safetyCompetencies/Pages/default.aspx (accesso Gennaio 2019).

23. Youngson GG, Flin R (2010). Patient safety in surgery: non-technical aspects of safe surgical performance. Patient Safety in Surgery;4:4. Disponib ile al sito: http://www.pssjournal.com/content/4/1/4 (accesso Gennaio 2019).

24. Gawande AA, Zinner MJ, Studdert DM, Brennan TA (2003). Analysis of errors reported by surgeons at three teaching hospitals. Surgery;133:614-21.

25. Leonard M, Graham S, Bonacum D. The human factor: the critical importance of effective teamwork and communication in providing safe care. Qual Saf Health Care. 2004 Oct;13 Suppl 1:i85-90.

26. Sutcliffe KM, Lewton E, Rosenthal MM (2004). Communication failures: An insidious contributor to medical mishaps. Acad Med;79:186-194.

27. Alvarez G, Coiera E (2006). Interdisciplinary communication: an uncharted source of medical error? J Crit Care;21:236-42.

28. Reader TW, Flin R, Cuthbertson BH (2007). Communication skills and error in the intensive care unit. Curr Op Critl Care;13:732-6.

29. Manser T, Harrison TK, Gaba DM, et al. (2009). Coordination patterns related to high clinical performance in a simulated anesthetic crisis. Anesth Anal;108: 1606-15.

30. The Joint Commission, 2015. Sentinel event Data. Root causes by event type: 2004-2014. Disponibile al sito: http://www.jointcommission.org/assets/1/18/Root_Caus es_by_Event_Type_2004-2014.pdf (accesso Gennaio 2019).

31. Mearns K, Flin R, O’Connor P (2001) Sharing 'worlds of risk'; improving communication with crew resource management, Journal of Risk Research;4(4):377-392.

32. Lee CTS \& Doran, DM (2017). The Role of Interpersonal Relations in Healthcare Team Communication and Patient Safety: A Proposed Model of Interpersonal Process in Teamwork. Canadian Journal of Nursing Research;49(2), 75-93.

33. Australian Medical Association. Safe handover: safe patients. AMA Clinical Handover Guide. AMA, Sydney, 2006. Disponibile al sito: http://ama.com.au/node (accesso Gennaio 2019).

34. Hu YY, Arriaga AF, Peyre SE, Corso KA, Roth EM, Greenberg CC (2012). Deconstructing intraoperative communication failures. J Surg Res;177(1):37-42.

35. Lingard L, Espin S, Whyte S, Regehr G, Baker GR, Reznick R, Bohnen J, Orser B, Doran D, Grober E (2004). Communication failures in the operating room: an observational classification of recurrent types and effects. Qual Saf Health Care;13(5):330-4.

36. CRICO Strategies. Malpractice risk in communication failures; 2015 Annual Benchmarking Report. Boston, Massachusetts: The Risk Management Foundation of the Harvard Medical Institutions, Inc., 2015.

37. Witherington EM1, Pirzada OM, Avery AJ (2008). Communication gaps and readmissions to hospital for patients aged 75 years and older: observational study. Qual Saf Health Care;17(1):71-5.

38. Salas, E., Sims, D. E., \& Shawn Burke, C. (2005). Is there a "Big Five" in Teamwork? Small Group Research, 36(5), 555-599.

39. Risser DT, Rice MM, Salisbury ML, Simon R, Jay GD, Berns SD (1999). The potential for improved teamwork to reduce medical errors in emergency department, Annals of Emergency Medicine, 34(3):373-83.

40. Boamah, S. A., Spence Laschinger, H. K., Wong, C., \& Clarke, S. (2018). Effect of transformational leadership on job satisfaction and patient safety outcomes. Nursing Outlook;66(2):180-189.

41. Cummings GG, Tatea K, Leea S, Wongb CA, Paananena T, Micaronia S, Chatterjeea GE (2018). Leadership styles and outcome patterns for the nursing workforce and work environment: A systematic review. International Journal of Nursing Studies;85:19-60.

42. Sexton JB, Thomas EJ, Helmreich RL (2000). Error, stress, and teamwork in medicine and aviation: cross sectional surveys. BMJ; 320(7237): 745-749.

43. Patterson PD, Weaver MD, Frank RC, Warner CW, Martin-Gill C, Guyette FX, Fairbanks RJ, Hubble MW, Songer TJ, Callaway CW, Kelsey SF, Hostler D (2012). Association between poor sleep, fatigue, and safety outcomes in emergency medical services providers. Prehosp Emerg Care;16(1):86-97.

44. Greena B, Oeppenb RS, Smithc DW, Brennand PA (2017). Challenging hierarchy in healthcare teams ways to flattengradients to improve teamwork and patient care. British Journal of Oral and Maxillofacial Surgery; 55:449-453.

45. Hedge J.W., Bruskiewicz K., Borman W.C., Hanson M.A., Logan K., Siem F (2000). Selecting pilots with crew resource management skills. International Journal of Aviation Psychology, 10(4), 377-92.

46. Ting WH, Peng FS, Lin HH, Hsiao SM (2017). The impact of situation-background-assessment-recommendation (SBAR) on safety attitudes in the obstetrics department. Taiwan J Obstet Gynecol;56(2):171-174.

47. Putnam LR, Anderson KT, Diffley MB, Hildebrandt AA, Caldwell KM, Minzenmayer AN, Covey SE, Kawaguchi AL, Lally KP, Tsao K (2016). Meaningful use and good catches: More appropriate metrics for checklist effectiveness. Surger;160(6):1675-1681.

48. Stead K, Kumar S, Schultz TJ, Tiver S, Pirone CJ, Adams RJ, Wareham CA (2009). Teams communicating through STEPPS. Med J Aust;190(11 Suppl):S128-32. 


\title{
Rischio clinico e carico di lavoro: alcuni spunti di riflessione
}

\author{
Paola Gnerre, ${ }^{1}$ Andrea Fontanella, ${ }^{2}$ Domenico Montemurro, ${ }^{3}$ Carlo Palermo ${ }^{4}$ \\ ${ }^{1}$ Dipartimento di Medicina Interna, Ospedale San Paolo, Savona; ${ }^{2}$ Presidente Nazionale FADOI; Dipartimento di Medicina, \\ Ospedale del Buon Consiglio Fatebenefratelli, Napoli; ${ }^{3}$ Direzione Medica Ospedale Madre Teresa di Calcutta, Monselice (PD); \\ ${ }^{4}$ Dipartimento di Medicina, Ospedale Poggibonsi (SI), Italia
}

La sicurezza dei pazienti è uno dei punti critici e pertanto uno degli obiettivi prioritari per tutti i sistemi sanitari e rappresenta uno degli elementi centrali per la promozione e la realizzazione delle politiche di governo clinico. Si stima che, nel mondo, circa 98 mila pazienti muoiano ogni anno in ospedale a causa di errori medici prevenibili. ${ }^{1}$ La maggior parte di questi errori sono conseguenza di processi o condizioni difettose del sistema e tra queste rientra l'eccessivo carico di lavoro degli operatori. ${ }^{2}$

Negli ultimi anni i Servizi Sanitari di tutti i paesi industrializzati hanno aumentato notevolmente il loro carico di lavoro per il crescente invecchiamento della popolazione con bisogni di salute complessi con difficoltà da parte dei servizi territoriali di fornire l'assistenza necessaria e conseguente aumentato ricorso ai servizi ospedalieri. L'analisi della King 's Fund inglese ha evidenziato un aumento del $17 \%$ degli accessi in Pronto Soccorso dal 2003/2004 al 2015/2016 un incremento dal $19 \%$ al $27 \%$ dei tassi di ammissione ospedaliera dagli anni 2003/04 agli anni 2015/16 e un incremento del tasso di occupazione posti letto pericolosamente alto (oltre $1^{\prime} 85 \%$ ). ${ }^{3}$ Questo incremento, di cui un terzo si è verificato negli ultimi due anni, rappresenta il singolo incremento più grande nel volume di assistenza all'interno di qualsiasi altro settore. ${ }^{3}$

Nonostante ciò le risorse destinate alla sanità sono rimaste pressoché stabili negli anni con il risultato che a parità di risorse l'aumento del numero delle prestazioni ha comportato l'incremento del carico lavorativo degli operatori, una deregulation dell'orario di lavoro e un peggioramento dell'integrità psicofisica degli

Corrispondente: Paola Gnerre, Dipartimento di Medicina, Interna, Ospedale San Paolo, Savona, Italia.

Tel.: +39.019.8404358 - Fax: +39.019.8404583.

E-mail: pgnerre@yahoo.it

Articolo pubblicato secondo la Creative Commons Attribution NonCommercial 4.0 License (CC BY-NC 4.0).

${ }^{\circ}$ Copyright: the Author(s), 2019

Licensee PAGEPress, Italy

QUADERNI - Italian Journal of Medicine 2019; 7(6):35-38 operatori con evidenti conseguenze sul rischio clinico. $^{3}$

Quando gli ospedali sono sovraccarichi con un tasso di occupazione posti letto che raggiunge la soglia di criticità i pazienti sono i primi a subirne le conseguenze in termini di mancanza di continuità clinica $\mathrm{e}$ di riduzione della qualità e della sicurezza delle cure. ${ }^{4}$ È ben noto come un ospedale con un indice di occupazione del posto letto $>90 \%$ abbia un limitato lavoro elettivo e un importante ritardo nella gestione di gravi malattie croniche. ${ }^{3}$ In mancanza di posti letto nei reparti specifici i pazienti vengono allocati in altri reparti realizzando il cosiddetto fenomeno degli appoggi noto a tutti come situazione ad alto rischio clinico. ${ }^{5} \mathrm{Un}$ fenomeno presente da almeno trent'anni, ma se associato al dato sulla contrazione dei posti letto (situazione particolarmente significativa in Italia) è facilmente intuibile come porti a tassi di occupazione sopra il $100 \%$ con veri e propri reparti duplicati in altri a iso-personale e al conseguente turn-over forzato. Malati medici si ritrovano ricoverati nelle degenze chirurgiche e viceversa, soggetti con elevati bisogni sono inseriti in un sistema a bassa offerta e soggetti con modesti bisogni sono inseriti in un sistema ad alta offerta in quanto l'allocazione dei pazienti non dipende più dalla loro patologia o condizione clinica, ma è conseguente alla necessità di trovare un posto letto con l'unico risultato evidente di stressare il sistema. Il malato in appoggio rischia di diventare un malato della terra di mezzo: giuridicamente appartiene ad un medico che non è fisicamente sempre presente e prontamente rintracciabile, assistenzialmente appartiene a degli infermieri che non sempre per tipologia e complessità di assistenza sono in grado di gestirlo. Il medico degli appoggi si trova a dover gestire un carico di pazienti aggiuntivo oltre a quelli tabellari e il paziente in appoggio rischia di essere vittima del sistema di cura anziché centro del sistema di cura. Tutto ciò si traduce in un sovraccarico di ansia e stress sia per il medico che percepisce un chiaro peggioramento delle condizioni lavorative sia da parte del paziente che subisce la frammentazione della cura con ben documentate ripercussioni sugli outcome clinici. Nell'attuale situazione il paziente in appoggio rappresenta di per sé una condizione ad alto 
rischio clinico inteso sia come condizione o evento potenziale sia come causa di errore attivo o di errore latente. La somministrazione di un farmaco sbagliato commesso da un operatore che ha poca dimestichezza con la tipologia del paziente da lui in appoggio rappresenta un errore attivo, ma è anche un errore latente in quanto conseguente ad un'insufficienza organizzativagestionale del sistema, che attraverso il fenomeno degli appoggi ha creato le condizioni favorevoli al verificarsi dell'errore. Come sostenuto da molti, quindi, la maggior parte degli errori è dovuta a carenze del sistema e non a negligenza dei singoli. ${ }^{5}$ In Tabella 1 è riportato un caso clinico di rischio conseguente a problematiche organizzative e ad un eccessivo carico lavorativo dell'operatore.

Il mantenere la salute psicofisica dell'operatore clinico e il garantire la sicurezza dell'assistenza ai pazienti devono essere obiettivi importanti per le organizzazioni sanitarie in quanto sono fenomeni intimamente correlati. La Direttiva 88/2003 CE sull'orario di lavoro rappresenta una tappa fondamentale del modello sociale europeo, poiché assicura una protezione minima a tutti i lavoratori contro orari di lavoro eccessivi e contro il mancato rispetto di periodi minimi di riposo. Nel 2008 in Italia è stata legalizzato una deregulation dell'orario di lavoro attraverso l'abrogazione per i soli dirigenti del SSN del limite massimo di lavoro giornaliero e settimanale e della normativa sui riposi giornalieri e settimanali. Dopo il deferimento del Governo Italiano alla Corte Europea, la legge del 30 Ottobre 2014 ha ripristinato la normativa, anche per i medici italiani. ${ }^{6}$ Una recente survey promossa da $\mathrm{ANAAO}^{6}$ ha comunque evidenziato, come quasi la metà degli intervistati non rispetti la normativa per esigenze di servizio. Ma la mancata fruizione del periodo di riposo o un eccessivo orario di lavoro può essere una scelta individuale magari anche imposta? La giurisprudenza e la sicurezza del paziente ci dicono di no. Il protrarsi dell'attività lavorativa in condizioni routinarie oltre l'orario di lavoro previsto dalle normative vigenti viene considerato in caso di evento avverso una condotta imprudente e costituisce un'aggravante ritenendosi come volontaria l'accettazione del turno irregolare e dei rischi connessi. ${ }^{7}$ La letteratura ci dimostra come eccessive ore lavorative oltre ad avere importanti ripercussioni sulla salute dell'operatore (aumentato rischio di puntura accidentale, aumentato rischio di malattie cardiovascolari e metaboliche, eventi cerebrovascolari e patologie neoplastiche, come il tumore della mammella nelle donne) possono avere importanti ricadute sulla salute del paziente. Eccessive ore lavorative e un eccessivo carico di lavoro specie nel periodo notturno inducono performance cognitive paragonabile a quelle che si hanno con un tasso alcolemico di $0,4-0,5 \% .{ }^{8}$ Pertanto l'eccessivo carico lavorativo ricade non solo su chi lo subisce ma su tutta la comunità. È curioso come un editoriale pubblicato su Lung oltre a riportare tutti gli effetti nefasti di un duro lavoro sulla salute degli operatori sanitari sottolinei come essendo la cultura di ore eccessive ben consolidata tra tutti i medici e quindi difficilmente cambiabile è la comunità che deve attuare e pretendere il cambiamento ideando nuove strategie di programmazione e pianificazione sanitarie per evitare che i rischi sanitari siano conseguenza di uno stato di fatica cronica degli operatori sanitari. ${ }^{7}$

Un incremento importante e prolungato dell'orario di lavoro e del carico di lavoro sono associati non solo ad un aumentato rischio di malattie organiche dell'operatore, ad un peggioramento oggettivo delle performance cognitive e quindi ad un incremento del rischio clinico per il paziente, ma anche a disturbi della sfera

Tabella 1. Caso clinico.

Paziente di 74 anni giunge in Pronto Soccorso per dispnea ingravescente. In anamnesi cardiopatia ischemica-ipertensiva sottoposta ad angioplastica. Paziente fumatore. Per il riscontro di versamento pleurico massivo a destra si decide di ricoverare il paziente. Per mancanza di posti letto in Medicina il paziente viene ricoverato in appoggio in Ortopedia. Il giorno successivo l'internista esegue toracentesi diagnostica. Vengono evacuati $1500 \mathrm{cc}$ di liquido giallo paglierino. L'internista richiede indagini chimico-fisiche da inviare al laboratorio analisi, indagini colturali da inviare alla microbiologia ospedaliera e indagine citologica da inviare in anatomia patologica. Esegue personalmente per via telematica le richieste per l'indagine chimico-fisiche e per le indagini colturali da inviare al laboratorio analisi e alla microbiologia ospedaliera. Esegue personalmente la richiesta cartacea dell'Anatomia Patologica (non era presente la possibilità di inviare la richiesta per via telematica) ma non segnala all'infermiera che la richiesta dell'anatomia patologica è cartacea e non specifica che i prelievi sono da inviare in tre posti differenti. L'internista eseguita la manovra si allontana dal reparto velocemente senza accertarsi che gli esami vengono inviati nei posti corretti perché deve vedere altri 7 pazienti in appoggio dislocati in reparti differenti oltre a dover visitare 12 pazienti degenti in corsia. Il paziente nel pomeriggio viene trasferito in Medicina e l'internista si accorge che non è stato inviato il campione in anatomia patologica. Il giorno successivo viene nuovamente eseguita toracentesi diagnostica per poter effettuare l'indagine citologica. La manovra è complicata dalla comparsa di pneumotorace. Viene segnalata la criticità al Coordinatore infermieristico e al Direttore dell'Ortopedia che vorrebbero intentare un procedimento disciplinare nei riguardi dell'infermiera che era incaricata all'invio dei campioni. Il gruppo di risk management aziendale decide invece di eseguire un audit. Nel corso dell'audit si mette in evidenza come alla base dell'errore siano presenti problematiche organizzative:

1. La toracentesi è un'indagine che non viene eseguita di routine in Ortopedia per cui gli infermieri non hanno dimestichezza nella procedura. L'errore commesso dall'infermiera era in parte conseguenza di un'errata allocazione del paziente dettata da esigenze organizzative.

2. L'internista ammette che non ha specificato dove dovessero essere inviati i tre campioni e non si è accertato che i campioni fossero inviati nei posti giusti non avendo tempo a sufficienza e dovendo ancora visitare altri pazienti in appoggio oltre ai pazienti degenti in corsia. L'errore è quindi anche conseguenza di un eccessivo carico lavorativo. 
psichica come la sindrome da burnout. ${ }^{8}$ Il burnout, tradotto letteralmente dall'inglese in bruciato, fuso, indica una condizione di esaurimento emotivo irrequietezza, apatia, depersonalizzazione e senso di frustrazione che colpisce prevalentemente gli operatori di professioni ad elevato investimento relazionale come quelli del settore sanitario. I medici o gli infermieri sottoposti a carichi di lavoro e stress eccessivi iniziano a perdere progressivamente l'empatia fino al raggiungimento della morte professionale, ossia la completa indifferenza verso la propria professione. E tutto ciò, oltre ad avere degli evidenti effetti drammatici sul piano individuale, ha degli indubbi effetti negativi particolarmente sul piano organizzativo e lavorativo con il calo della qualità del servizio, il calo della performance e l'aumento dell'assenteismo. Nonostante in letteratura pochi studi indaghino la correlazione obiettiva tra burn out dell'operatore e sicurezza del paziente alcuni lavori dimostrano come medici e infermieri affetti da burn out riportano più eventi avversi. L'operatore in burn out non ha più le energie necessarie per affrontare il proprio lavoro e potrebbe non investire l'energia necessaria a fornire assistenza sicura al paziente, ad esempio trascurando l'igiene delle mani o evitando il doppio controllo durante la preparazione del farmaco. Potrebbe anche essere meno attento e pronto ad affrontare situazioni impreviste come il peggioramento repentino della salute del paziente. La realizzazione personale è concettualmente vicino all'auto-efficacia (self-efficacy), vale a dire la convinzione di essere in grado di svolgere un compito contribuisce allo svolgere con successo un compito impegnativo. Le persone con self efficacy hanno prestazioni migliori perché vedono il compito da svolgere come una sfida piuttosto che una minaccia. Le persone affette da burn out hanno una netta diminuzione del senso di realizzazione personale e della convinzione di poter completare il proprio compito dando un contributo significativo. ${ }^{9}$ In una recente survey, $L a-$ vorare sotto pressione, promossa dal Royal College of Physicians rivolta ai medici inglesi l' $82 \%$ degli intervistati riteneva il personale sanitario estremamente demoralizzato dall'eccessivo carico lavorativo conseguente alla carenza di personale sia medico che infermieristico. Il 55\% dei medici riteneva che la sicurezza del paziente si fosse ridotta negli ultimi 12 mesi per un contemporaneo notevole peggioramento della qualità delle cure e il $74 \%$ affermava quindi di essere estremamente preoccupato per l'assistenza che avrebbe potuto fornire ai pazienti nei successivi 12 mesi. $^{3}$ Anche una recente survey italiana promossa dall'ANAAO, sindacato medico, aveva evidenziato alti tassi di burn out tra gli operatori sanitari italiani. ${ }^{10-12}$

In un sistema di organizzazione industriale inteso come un insieme non solo di uomini ma anche di macchine e attrezzature finalizzato alla produzione e al profitto l'aumento del carico lavorativo può sicura- mente avere indubbi vantaggi. Ma in ambito lavorativo dove la forza lavoro è fatta solo di persone e il prodotto finito è il paziente questa programmazione lavorativa comporta solo evidenti criticità. In conclusione, la sicurezza delle cure è uno specifico processo che mira a evitare, prevenire e mitigare effetti avversi o danni derivanti dal processo di assistenza sanitaria. L'eccessivo carico di lavoro degli operatori mette a repentaglio la sicurezza delle cure che essendo parte costitutiva del diritto alla salute deve essere sempre perseguita nell'interesse soprattutto dell'individuo e della collettività. ${ }^{7}$

\section{Bibliografia}

1. Weigl M, Muller A, Vincent C, et al. The association of workflow interruptions and hospital doctors' workload: a prospective observational study. BMJ Qual Saf 2012.

2. Measuring Patient Harm in Canadian Hospitals October 2016. Available from: https://www.google.it/search?ei $=$ rwaJW_LeL87prgTQxqTwDA\&q=Measuring $+\mathrm{Pa}-$ tient+Harm+in+Canadian+Hospitals+October+2016\&oq $=$ Measuring + Patient + Harm + in + Canadian + Hospitals + Oc tober +2016

3. Royal College of Physicians. NHS reality check Delivering care under pressure. Available from: https:// www.rcplondon.ac.uk/projects/nhs-reality-check-delivering-care-under-pressure

4. Quality first: Managing workload to deliver safe patient care January 2015. Available from: https://www.google. it $/$ search?ei=8QaJWEKSRrgSp7A4\&q=Quality+first $\%$ $3 \mathrm{~A}+$ Managing + workload + to + deliver + safe + patient + car e+January +2015

5. Pronto Soccorso/2. Il fenomeno degli "appoggi": un rischio per medici e pazienti. Autori Paola Gnerre e Domenico Montemurro pubblicato su Quotidianosanità.it Mercoledì 11 Febbraio 2015.

6. Gnerre P, Montemurro D, Rossi AP, et al. Lack of application of The European Work Time Directive: effects on workload, work satisfaction and burnout among italian physicians. Ital J Med 2017;11;159-63.

7. Gnerre P, Montemurro D, Troise C, Palermo C. Il lavoro ai tempi del Burn out. pubblicato sul Sole 24 ore Sanità il 18 novembre e su Dirigenza Medica (Marzo 2015).

8. Arnedt JT, Owens J, Crouch M, et al. Neurobehavioral Performance of Residents After Heavy Night Call vs After Alcohol Ingestion. JAMA 2005;294:1025-33.

9. GnerreP, Rivetti C, Rossi AP, et al. Work stress and burnout among physicians and nurses in internal and emergency departments. Ital J Med 2017;11;151-8.

10. Rossi A, Gnerre P, Amati D, et al. Medici se il lavoro in corsia diventa una guerra. La survey di ANAAO giovani. Sole 24 ore sanità. 14 Aprile 2015 e Dirigenza Medica (Aprile 2015).

11. World Health Organization. Conceptual Framework for the International Classification for Patient Safety; 2009.

12. Welp A, Meier LL, Manser T. Emotional exhaustion and workload predict clinician-rated and objective patient safety. Front Psychol 2015;5:1573. 


\title{
Il punto di vista di un Direttore Generale internista
}

\author{
Gianluigi Scannapieco, Eleonora Croci \\ IRCCS Burlo Garofolo, Trieste, Italia
}

\section{Introduzione}

La sicurezza dei pazienti è una priorità che gli Enti dei Servizi Sanitari Regionali (SSR) pongono a garanzia della qualità e dell'equità delle cure. Per il suo perseguimento è necessario l'impegno di tutti, attraverso programmi di Governo Clinico e di miglioramento continuo della Qualità che pongono al centro le necessità dei cittadini e valorizzano il ruolo e la responsabilità di tutte le figure professionali che operano all'interno del sistema.

Garantire la sicurezza delle cure ai pazienti attraverso la prevenzione e la gestione del rischio clinico richiede, per le organizzazioni sanitarie, un profondo e radicale cambiamento della cultura organizzativa e professionale che trasformi l'errore da qualcosa da nascondere per il timore delle conseguenze, ad una risorsa da utilizzare ed analizzare per migliorare le conoscenze e adottare misure correttive volte a ridurre la probabilità che si ripeta. La necessità di strutturare un sistema organizzativo per affrontare il tema della sicurezza dei pazienti emerge da raccomandazioni del Ministero della Salute Italiano e dell'Unione Europea oltre che da evidenze scientifiche sulla necessità di una funzione per il coordinamento centrale dello stesso.

Una corretta ed efficace Gestione del Rischio Clinico dipende sicuramente dal grado di consapevolezza dei medici e del personale sanitario, ma non può prescindere da un adeguato modello organizzativo che veda coinvolta in modo organico e integrato l'intera filiera Regione-Aziende Sanitarie-professionisti-cittadini.

Corrispondente: Gianluigi Scannapieco, IRCCS Burlo Garofolo, Trieste, Italia.

E-mail: gianluigi.scannapieco@gmail.com

Articolo pubblicato secondo la Creative Commons Attribution NonCommercial 4.0 License (CC BY-NC 4.0).

${ }^{\circ}$ Copyright: the Author(s), 2019

Licensee PAGEPress, Italy

QUADERNI - Italian Journal of Medicine 2019; 7(6):39-42

\section{Livello regionale}

Molte Regioni hanno sviluppato modelli organizzativi per la gestione integrata del rischio clinico. Le dimensioni della Regione Friuli Venezia Giulia (FVG) consentono di affrontare la Gestione del Rischio Clinico attraverso un' organizzazione unitaria, in accordo con le linee d'indirizzo e con i programmi sviluppati, a livello nazionale, dal Ministero della Salute, dall'Agenzia Nazionale per i Servizi Sanitari Regionali (AGENAS), dal tavolo tecnico specifico presso la Conferenza Stato-Regioni e, a livello europeo, dall'Unione Europea e dall'European Centre for Disease Prevention and Control (ECDC).

La filosofia di lavoro prevede un forte indirizzo e coordinamento regionale accompagnato dalla promozione e valorizzazione delle iniziative delle Aziende del SSR, degli IRCCS e delle Case di Cura private accreditate convenzionate. Questa sinergia si declina attraverso incontri mensili con i referenti aziendali (Risk Manager), con la costituzione di una rete di Referenti Aziendali per l'attuazione dei singoli programmi, con l'attivazione di gruppi di esperti coinvolti di volta in volta su specifiche tematiche e con il coinvolgimento finalizzato dei cittadini.

I programmi riguardano l'uso sicuro dei farmaci, il sistema di segnalazione di incident reporting e l'applicazione di strumenti di analisi degli eventi avversi più gravi, la corretta identificazione del paziente, la sicurezza del percorso chirurgico, la qualità e sicurezza della documentazione sanitaria, l'informazione al paziente e l'acquisizione del consenso informato, la prevenzione e il controllo delle infezioni correlate all'assistenza, l'uso corretto degli antibiotici (antibiotic stewardship), la prevenzione delle lesioni da pressione, la prevenzione delle cadute accidentali, il controllo del dolore, la promozione delle buone pratiche e la promozione del coinvolgimento attivo e consapevole dei pazienti/cittadini.

Nel 2016 è stata istituita la Rete Cure Sicure FVG, coordinata da un Comitato Strategico Regionale, che nasce con il fine di garantire la sicurezza di tutte le prestazioni sanitarie, dal singolo esame diagnostico al percorso assistenziale. Attraverso la Rete, la Regione coordina e governa, in forma integrata, le attività connesse alla sicurezza dei pazienti, dalla 
prevenzione del rischio clinico alla gestione dei sinistri. Peculiarità della rete è lo sviluppo e la condivisione di competenze e conoscenze di una comunità interdisciplinare di esperti che, grazie allo scambio continuo delle informazioni e delle esperienze, producono innovazione e perseguono il miglioramento dell'assistenza.

La Rete Regionale Cure Sicure, oltre a definire gli organi e le relative funzioni a livello regionale, stabilisce anche la struttura e le funzioni interne alle singole Aziende in un modello integrato e dialogante tra i diversi livelli di attività e di responsabilità.

\section{Livello aziendale}

Altrettanto fondamentale è il modello organizzativo aziendale che deve prevedere uffici/strutture di riferimento ben individuate all'interno dell'Azienda, coordinata da un referente aziendale (Risk Manager) e collegate ad una rete di referenti per le singole strutture aziendali (Link Professional).

A livello aziendale la funzione di Rischio Clinico deve prevedere personale dedicato e formato, deve essere nota agli operatori e deve condividere attività, programmi, progetti, obiettivi con i diversi servizi, i team di professionisti e i singoli operatori. Tutto ciò avviene grazie all'individuazione e alla formalizzazione dei Link Professionals, i Referenti di Struttura, medici e non, individuati in base alle specifiche competenze e attitudini ma anche ai ruoli interni ai servizi: rappresentano da un lato vie preferenziali e dirette per comunicare e aggiornare i temi e le novità al personale operante all'interno dei servizi e delle strutture e dall'altro i portavoce e i segnalatori di criticità, urgenze o altro propri del servizio o del momento o del contesto specifico di attività.

In ogni Azienda viene quindi individuato il Risk Manager Aziendale, figura fiduciaria del Direttore Generale, che ha funzioni di raccordo fra il livello regionale e quello aziendale. Il Risk Manager supporta tutte le attività relative alla gestione del rischio clinico a livello aziendale, coordinando la Rete Aziendale dei Link Professionals, attivando la formazione, organizzando specifici audit per la verifica sul campo degli eventi avversi, seguita dalla stesura di un report con raccomandazioni per gli operatori e piano delle azioni di miglioramento con successivo monitoraggio dei risultati raggiunti.

La centralità del tema e la sua priorità all'interno della programmazione delle attività regionali è evidenziata dalla presenza e dalla declinazione, nelle linee annuali di gestione del SSR del FVG, di specifici obiettivi inerenti la qualità e la sicurezza della cure, aggiornati ogni anno, con la chiara definizione di azioni e attività implementate e monitorate ed eventualmente modificate, attraverso un'attenta e precisa definizione di indicatori facilmente misurabili e monitorabili. A livello aziendale, le linee di gestione regionali in tema di sicurezza e qualità delle cure vengono declinate in un piano dettagliato e specifico allegato al Piano Attuativo Ospedaliero (PAO), trasversale alle strutture aziendali, adattato alla realtà aziendale in base ai risultati ottenuti, alle peculiarità dell'attività clinico-assistenziale, alle criticità individuate dai professionisti operanti all'interno dell'Istituto. Gli obiettivi del PAO vengono declinati nelle schede di budget delle singole strutture e incentivati in base alla loro rilevanza all'interno del singolo servizio o dello specifico percorso, per sensibilizzare e responsabilizzare gli operatori sanitari e collegare i comportamenti virtuosi ad un sistema premiante.

Oltre alla individuazione di linee di lavoro su specifiche tematiche, all'elaborazione di Linee Guida Regionali condivise e adattate al contesto regionale, è fondamentale costruire, introdurre, adeguare, aggiornare e monitorare strumenti informatici, utili a ridurre e contenere i rischi, quali la Cartella Clinica Informatizzata e il Fascicolo Sanitario Elettronico, per rendere immediatamente fruibili ed accessibili le informazioni e i dati sanitari di rilievo del singolo cittadino da qualsiasi punto di erogazione del sistema. Altri applicativi specifici per singole linee di lavoro sono l'invio automatico di messaggistica relativa all'isolamento di agenti patogeni alert, per la segnalazione immediata alla struttura di degenza inviante e la possibilità di allegare alla lettera di dimissione schede informative per i pazienti, i familiari e i caregivers, relative ai rischi individuali e specifici (cadute, insorgenza di lesioni da pressione, rischio tromboembolico, colonizzazione da parte di agenti patogeni multiresistenti, etc.).

\section{Collegamento con la gestione sinistri}

Per rendere efficace ed efficiente la Gestione del Rischio Clinico è fondamentale uno stretto collegamento con la Gestione Sinistri, garantita a livello regionale da un apposito Comitato Regionale e, a livello aziendale, dal Comitato Aziendale per la Gestione Sinistri, composto da Risk Manager, Referente Gestione Amministrativa e Legale, Referente Medico Legale, Referente della Direzione Sanitaria, Esperto di Liquidazione Sinistri. In tal modo si riesce a garantire uno stretto collegamento fra la Gestione del Rischio Clinico e quella degli eventi conseguenti a denunce, che spesso seguono percorsi paralleli e non comunicanti.

\section{Coinvolgimento dei cittadini e delle associazioni}

I cittadini entrano a far parte della Rete Cure Sicure attraverso gli Uffici Relazioni con il Pubblico, at- 
traverso il Tavolo di Alleanza Permanente e la partecipazione al Comitato strategico regionale. Le finalità del Tavolo di Alleanza Permanente con i cittadini sono: definire e presentare le criticità riguardanti il cittadino proponendo temi di rilievo, identificare strategie per migliorare il coinvolgimento e la consapevolezza dei cittadini (empowerment) sulle decisioni che riguardano la salute, revisionare e validare la documentazione prodotta, allo scopo di raggiungere una comunicazione più chiara e diretta con il cittadino.

\section{Attività formativa}

Ultimo ma non meno importante punto fermo della Gestione del Rischio Clinico è la programmazione della formazione, sia a livello regionale che aziendale, dedicata al Rischio Clinico, fondamentale per far crescere nei professionisti sanitari la sensibilità verso queste tematiche.

A livello regionale alcune linee di interesse comune vengono inserite nel Piano Regionale della Formazione ed affidate alle singole Aziende, con il compito di organizzare l'evento formativo rivolto a tutte le aziende del SSR: si tratta di eventi formativi residenziali o a distanza su tematiche di nuova introduzione all'interno dei programmi e progetti della Rete Regionale Cure Sicure o comunque di interesse trasversale oppure dedicati alla presentazione di nuove linee guida, protocolli e procedure operative regionali.

A livello aziendale, il Piano Formativo viene redatto sulla base delle necessità e delle criticità locali e peculiari, tenuto conto del raggiungimento degli obiettivi, degli indicatori, delle attività di controllo e monitoraggio, delle segnalazioni di eventi avversi o quasi eventi ricevuti dalla cui analisi è emersa una o più criticità correggibili con percorsi di formazione, aggiornamento o addestramento professionale e dall'analisi del fabbisogno formativo del Referente di Struttura della Formazione e dei Link Professionals.

\section{Riferimenti normativi}

\section{Normativa europea}

- Consiglio d'Europa: Raccomandazione n. R (1997) 17 del Comitato dei Ministri agli Stati Membri - "Sullo Sviluppo e l'attivazione di Sistemi di Miglioramento della Qualità (SMQ) dell'Assistenza Sanitaria".

- Consiglio d'Europa: Raccomandazione n. C (2009) 151/01 del Consiglio - "Sulla sicurezza dei pazienti, comprese la prevenzione e il controllo delle infezioni associate all'assistenza sanitaria".
- Consiglio d'Europa: Conclusione n. C (2014) 438/05 del Consiglio - "Conclusioni del Consiglio sulla sicurezza dei pazienti e la qualità dell'assistenza medica, compresi la prevenzione e il controllo delle infezioni associate all'assistenza sanitaria e della resistenza agli antimicrobici”.

\section{Normativa italiana}

- D. Lgs. 30 dicembre 1992, n. 502 "Riordino della disciplina in materia sanitaria, a norma dell'art. 1 della Legge 23 ottobre1992, n. 421".

- D.P.R. 14 gennaio 1997, n. 37 "Approvazione dell'atto di indirizzo e coordinamento alle regioni e alle province autonome di Trento e Bolzano, in materia di requisiti strutturali, tecnologici ed organizzativi minimi per l'esercizio delle attività sanitarie da parte delle strutture pubbliche e private".

- D. Lgs. 19 giugno 1999, n. 229 "Norme per la razionalizzazione del Servizio sanitario nazionale, a norma dell'articolo 1 della legge 30 novembre 1998, n. 419".

- Decreto Ministero della Salute 2 aprile 2015, n. 70 "Regolamento recante definizione degli standard qualitativi, strutturali, tecnologici e quantitativi relativi all'assistenza ospedalieri".

- Legge 28 dicembre 2015, n. 208 "Disposizioni per la formazione del bilancio annuale e pluriennale dello Stato".

- Ministero del Lavoro, della Salute e delle Politiche sociali "Protocollo per il monitoraggio degli eventi sentinella". Disponibile presso: http://www.salute.gov.it/imgs/c_17_pubblicazioni_1783_allegato.pdf

\section{Normativa regionale FVG}

- Legge regionale n. 12 del 23 luglio 2009 “Assestamento del bilancio 2009 e del bilancio pluriennale per gli anni 2009-2011 ai sensi dell'articolo 34 della legge regionale 21/2007".

- D.G.R. n. 465 dell' 11 marzo 2010 “Lr 23/2004 art 8 - approvazione definitiva del piano sanitario e sociosanitario regionale 2010-2012".

- Legge regionale n. 17 del 16 ottobre 2014 "Riordino dell'assetto istituzionale e organizzativo del Servizio sanitario regionale e norme in materia di programmazione sanitaria e sociosanitaria".

- D.G.R. n. 1363 del 15 luglio 2015 "Lr 49/1996, art 16 e art 20 - Programmazione annuale 2015 del Servizio Sanitario Regionale".

- D.G.R. n. 1970 del 21 ottobre 2016: "LR 208/2015 - Rete Cure Sicure della Regione Friuli Venezia Giulia: modello organizzativo". 


\title{
Il punto di vista di un Direttore Sanitario internista
}

\author{
Francesco Orlandini \\ Direzione Sanitaria, ASL4 Liguria, Chiavari (GE), Italia
}

\section{Premessa}

È noto che il direttore sanitario è il responsabile della Clinical Governance aziendale che include fra $\mathrm{i}$ suoi pilastri la gestione del rischio clinico. ${ }^{1} \mathrm{Il}$ ruolo delicato del direttore sanitario è di fatto creare l'ambiente in cui la cultura della sicurezza possa diffondersi e radicarsi per determinare il miglioramento.

Nel nostro paese, tuttavia, il livello di cultura della sicurezza presente nelle varie aziende sanitarie non è sempre elevato. Poche sono le realtà che hanno raggiunto il livello cosiddetto generativo in cui la sicurezza permea le decisioni organizzative o proattivo in cui gli errori vengono anticipati e corretti, molte di più sono le realtà in cui la cultura della sicurezza è a uno stadio sistematico in cui sono stati messi in piedi una serie di sistemi per controllare il rischio e reattivo in cui il sistema si attiva solo dopo un evento, o addirittura inconsapevole. Negli ultimi tempi, l'ideale è la cosiddetta just Culture che premia i comportamenti che vanno oltre le aspettative, offre supporto in caso di errore umano e coaching nelle situazioni a rischio, ma non tollera le violazioni deliberate. ${ }^{2}$

Il manuale ministeriale per la formazione degli operatori sanitari in tema di sicurezza dei pazienti già nel $2009^{3}$ recitava a proposito della gestione del rischio: i) deve far parte della vision aziendale, di dipartimento, di unità operativa e deve essere previsto nelle strategie e nelle direttive e non lasciato esclusivamente alla buona volontà dei singoli. Infatti, soltanto azioni sinergiche potranno risultare in esiti ed in sicurezza per tutti: cittadini utenti, personale, visitatori ed altri soggetti che frequentano i servizi; ii) è un aspetto tecnico (e non solo generica buona vo-

Corrispondente: Francesco Orlandini, Direzione Sanitaria, ASL4 Liguria, Chiavari (GE), Italia.

E-mail: orlandinif@libero.it

Articolo pubblicato secondo la Creative Commons Attribution NonCommercial 4.0 License (CC BY-NC 4.0).

${ }^{\circ}$ Copyright: the Author(s), 2019

Licensee PAGEPress, Italy

QUADERNI - Italian Journal of Medicine 2019; 7(6):43-44 lontà) che richiede competenza per effettuare scelte pertinenti rispetto alla singola persona e l'adozione e l'impiego appropriato di metodologie e strumenti; iii) deve far parte della formazione di inserimento dei nuovi assunti/trasferiti nei diversi servizi, oltre che di quella di base e continua degli operatori.

All'atto pratico ${ }^{4}$ ciò corrisponde a:

- considerare l'errore un difetto di sistema e non responsabilità del singolo operatore per cui vengono prescritti procedimenti disciplinari o altri provvedimenti (es. trasferimenti d'ufficio) degli operatori coinvolti;

- dare priorità alla sicurezza rispetto agli obiettivi economico-finanziari;

- incoraggiare e premiare l'identificazione, la segnalazione e la risoluzione dei problemi di sicurezza; sfruttare il potenziale di apprendimento dagli errori;

- fornire risorse, strutture e mezzi per costruire e mantenere un sistema di sicurezza efficace;

- pianificare e implementare programmi aziendali per la gestione del rischio, mediante il coordinamento del risk manager.

Infine, il direttore sanitario, in molte aziende, presiede l'unità di crisi che si riunisce in caso di evento sentinella ed è il portavoce verso l'esterno (media, associazioni di tutela dei pazienti, ecc.) in tali situazioni.

\section{Come promuovere la sicurezza dei pazienti in medicina interna}

Per quanto attiene specificatamente la medicina interna, l'impegno per la sicurezza della Direzione Sanitaria si tradurrà nel promuovere, oltre alla formazione degli operatori: i) la revisione dei percorsi di cura mediante audit clinici; ii) azioni per la prevenzione/controllo dei principali rischi del paziente internistico.

A tal proposito, si rimanda alle Top Patient Safety Strategies, pubblicate da Annals of Internal Medicine nel 2013, ${ }^{5}$ distinte in pratiche da incoraggiare e pratiche da incoraggiare fortemente in base alla forza delle evidenze a supporto. Molte di esse interessano i reparti di medicina interna: interventi per ridurre l'uso del catetere vescicale, strategie di prevenzione delle infezioni correlate ai cateteri venosi centrali, uso della 
guida ecografica per il posizionamento dei CVC, igiene delle mani e precauzioni di barriera per prevenire le infezioni correlate all'assistenza, profilassi del tromboembolismo venoso, interventi multicomponenti per prevenire le ulcere da pressione e le cadute, farmacisti clinici per ridurre gli eventi avversi da farmaci, documentazione delle volontà di fine vita dei pazienti, consenso informato, riconciliazione farmacologica, interventi per ridurre gli esami radiologici, formazione mediante simulazione, farmaco-prescrizione elettronica.

Altri rischi non trascurabili in medicina interna sono: ab ingestis, delirium, ventiloterapia, errore/ritardo diagnostico.

Nelle infezioni correlate all'assistenza la responsabilità organizzativa della direzione aziendale, infatti, supera di gran lunga la responsabilità del personale medico. Se al medico spetta la diagnosi precoce e corretta e la terapia adeguata, la direzione aziendale è responsabile della predisposizione di strutture di monitoraggio e intervento (es. Comitato infezioni ospedaliere), di procedure e protocolli destinati agli operatori di prima linea sulla prevenzione e gestione di tali infezioni, ma anche di procedure e protocolli per garantire e monitorare la profilassi ambientale (disinfezione ambienti, sterilizzazioni, qualità impianti, ecc.).

\section{Le sfide più recenti}

I problemi più spinosi, nonché vere e proprie minacce per la qualità e sicurezza delle cure erogate dai reparti di medicina interna sono attualmente: i) carenza di personale; ii) sovraffollamento leggi ricoveri sovrannumerari (su barelle, letti aggiunti o fuori reparto); iii) frammentazione delle cure dei pazienti poli-patologici; iv) danni da politerapia (interazioni farmaco-farmaco; farmaco-malattia, effetti colinergici, ecc).

Le medicine interne, considerate a lungo reparti a bassa complessità, ma naturale destinazione dei pazienti poli-patologici in crescente aumento nella popolazione ospedaliera, presentano attualmente staff (medici, infermieri e personale di supporto) non adeguati e tale carenza è aggravata dall'assenza di standard di riferimento e dai ricoveri sovrannumerari che, nel nostro paese, superano di gran lunga il tasso del 7$8 \%$ riportato in letteratura mettendo in crisi un'assistenza già insufficiente. ${ }^{6}$

L'esperienza ospedaliera dei pazienti polipatologici è spesso costellata da numerosi trasferimenti fra reparti subspecialistici con perdita di informazioni cliniche, ripetizioni di esami, decisioni non ponderate e senso di smarrimento per i pazienti, orfani di una figura di riferimento.
Da quanto esposto risulta evidente che si tratta di problemi che investono sì le Medicine interne, ma difficilmente risolvibili al loro interno in quanto determinati da fattori sistemici (criteri aziendali di distribuzione del personale e dei posti letto, appropriatezza di ricovero, collaborazione fra unità operative, suddivisione dell'ospedale basata sulle specialità, ecc). Solo la direzione sanitaria con la sua visione sistemica può individuare e correggere i determinanti locali dei ricoveri sovrannumerari, modificare le dimensioni di uno staff o disegnare e implementare nuovi modelli organizzativi, basati sugli internisti, per dare unitarietà alla cura dei pazienti polipatologici e razionalizzare la politerapia.

\section{Conclusioni}

Se la Direzione Generale detta le politiche generali necessarie a garantire adeguati livelli di sicurezza, in base alle indicazioni nazionali e regionali, la Direzione Sanitaria definisce priorità e risorse necessarie, supervisiona le attività di gestione del rischio, ma soprattutto crea l'ambiente favorevole alla cultura della sicurezza che riduce gli eventi, migliora gli outcome per i pazienti, migliora la soddisfazione di pazienti e operatori e riduce gli sprechi (meno ospedalizzazioni, meno complicanze, meno contenziosi, maggiore appropriatezza, ecc.). Esiste tuttavia un grosso limite in questo processo: la durata del mandato di un direttore sanitario è di gran lunga più breve del tempo necessario per produrre i risultati delle attività promosse, soprattutto se questi implicano un cambiamento di mentalità o comportamenti.

\section{Bibliografia}

1. GIMBE Position Statement. Il governo clinico nelle aziende sanitarie; 2009.

2. Boysen PG, Just Culture: A Foundation for Balanced Accountability and Patient Safety, Ochsner J. 2013;13:400-6.

3. Ministero della Salute, Sicurezza dei pazienti e gestione del rischio clinico: manuale per la formazione degli operatori sanitari, disponibile al seguente URL: http:// www.salute.gov.it/imgs/C_17_pubblicazioni_640_allegato.pdf

4. WHO, Patient safety, available at: https://www.who.int/ patientsafety/education/curriculum/coursela handout.pdf

5. Shekelle PG et al. The Top Patient Safety Strategies That Can Be Encouraged for Adoption Now. Ann Intern Med 2013;158:365-8.

6. La Regina M, Guarneri F, Romano E, et al. What quality and safety of care for patients admitted to clinically inappropriate wards: a systematic review. J Gen Int Med (in press). 


\title{
Il punto di vista di un Direttore Sanitario igienista
}

\author{
Chiara Bovo \\ Direttore Sanitario, Azienda Ospedaliera Universitaria Integrata di Verona, Italia
}

La sicurezza delle cure ha rappresentato negli ultimi decenni uno dei temi principali affrontati in sanità pubblica anche se talora ha assunto una deriva di sistema eccessivamente sbilanciata sul controllo degli aspetti assicurativi e di gestione della sinistrosità, nonché verso il contrasto ad una medicina difensiva in progressiva e costante espansione di fronte alla minaccia di un contenzioso in aumento con l'aumentare della complessità clinica della medicina moderna. Ciò ha spesso determinato un apparente scollamento tra le istanze del clinico e gli obiettivi delle Direzioni strategiche nei contesti aziendali, in parte giustificato anche da una disomogenea cultura manageriale tra le varie componenti ed, in particolare, nella difficile declinazione del ruolo del middle management all'interno della compagine organizzativa. Nel contesto ospedaliero la recente evoluzione verso i modelli organizzativi per intensità di cura e la ricerca di livelli di appropriatezza clinico-organizzativa sempre maggiori hanno comportato notevoli benefici sul piano dell'efficienza e dell'efficacia, imponendo tuttavia una maggiore attenzione alla gestione della sicurezza. La riforma sanitaria, sin dal 1992 aveva tracciato la strada per la definizione dei requisiti strutturali-organizzativi per l'esercizio delle attività sanitarie, ${ }^{1}$ prevedendo tra gli obiettivi generali quello di garantire l'osservanza delle norme nazionali (...) anche al fine di assicurare condizioni di sicurezza agli operatori e agli utenti dei servizi. Allo stato attuale, la Legge 24/2017, afferma che la sicurezza delle cure si realizza (...) mediante l'insieme di tutte le attività finalizzate alla prevenzione e alla gestione del rischio connesso all'erogazione di prestazioni sanitarie e l'utilizzo appropriato delle ri-

Corrispondente: Chiara Bovo, Azienda Ospedaliera Universitaria Integrata di Verona, piazzale Aristide Stefani 1, Verona, Italia.

Tel.: +39.0458.122270

E-mail: direzione.sanitaria@aovr.veneto.it

Articolo pubblicato secondo la Creative Commons Attribution NonCommercial 4.0 License (CC BY-NC 4.0).

${ }^{\circ}$ Copyright: the Author(s), 2019

Licensee PAGEPress, Italy

QUADERNI - Italian Journal of Medicine 2019; 7(6):45-48 sorse strutturali, tecnologiche e organizzative. ${ }^{2}$ È indubbio come tali riferimenti normativi richiamino in maniera diretta le responsabilità del management sanitario in merito all'identificazione e alla prevenzione dei rischi associati alle cure nel contesto delle Aziende Sanitarie. Tali responsabilità si esplicano principalmente nell'osservanza dei requisiti organizzativi e strutturali per l'erogazione delle cure (accreditamento), nel monitoraggio di qualità e appropriatezza dei percorsi e in un'adeguata gestione della documentazione sanitaria e dei flussi informativi.

In tale prospettiva, il Risk Management rappresenta primariamente uno strumento irrinunciabile della clinical governance, volto a ridurre l'incidenza di eventi avversi e a migliorare la qualità dei percorsi assistenziali. Bizzarri e Farina (2012) ${ }^{3}$ analizzano i possibili approcci allo sviluppo di una gestione strategica del rischio nelle organizzazioni sanitarie, sottolineando la necessità di considerare la relazione tra la creazione di valore e l'impatto nella gestione. La correlazione tra questi due aspetti è riconducibile a tre possibili configurazioni: la gestione finanziaria del rischio (gestione assicurativa), la gestione a livello di Unità Operativa e la gestione a livello aziendale.

È inoltre necessaria l'integrazione del rischio nella pianificazione e nella programmazione aziendale, attraverso l'elaborazione di un piano di settore comprendente gli obiettivi strategici correlati al rischio clinico, spesso inseriti nel piano delle performance e nelle politiche di budget aziendale nell'area degli obiettivi riservata alla qualità dell'assistenza. Tale programmazione deve necessariamente rifarsi anche ai modelli di gestione promulgati a livello delle singole Regioni, che risulteranno d'ausilio in merito alla definizione delle responsabilità e delle modalità operative per i diversi attori coinvolti.

Nel caso della Regione Veneto ad esempio, il ruolo del Direttore Sanitario è richiamato dalla DGR 2255/2016, che prevede l'istituzione di una Unità di Analisi in caso di evento avverso ad alta gravità, la cui composizione è di volta in volta definita in base alle figure professionali/dirigenziali ritenute più opportune. ${ }^{4}$ Il notevole impegno associato ad una gestione strategica (e sistemica) del rischio richiede pertanto risorse professionali dedicate e di supporto 
alla figura del Direttore Sanitario (e ad esso strettamente correlate), che agisce in qualità di promotore $\mathrm{e}$ di garante della qualità assistenziale dei percorsi erogati. Allo stato attuale in Veneto, tale funzione è attribuita alla figura del Risk Manager, posto in staff alla Direzione Sanitaria, il cui profilo di competenze è specificato peraltro dalla stessa Legge Gelli-Bianco. Il contributo del Risk Manager assume una valenza prevalentemente tecnica (applicazione degli strumenti di mappatura e gestione del rischio, gestione del contenzioso, formazione...) e i risultati conseguiti devono essere valutati periodicamente attraverso indicatori specifici e misurabili. Le attività di gestione del rischio sono caratterizzate da un forte coinvolgimento della componente clinica (rischio clinico), e debbono prevedere la definizione di una rete di referenti per la sicurezza presso le singole Unità Operative in un'ottica di tipo sistemico. Può inoltre avvalersi per gli aspetti di valutazione e condivisione del Piano annuale per la sicurezza e quindi delle azioni prioritarie da programmare e monitorare in tale ambito di un Comitato aziendale per la sicurezza del paziente in cui, accanto alla componente tecnica (Risk manager, direttore medico, medico legale, avvocato, farmacista, ecc.) siano rappresentate le principali aree cliniche (medica, chirurgica, intensiva, dell'emergenza-urgenza ecc.).

Il Direttore Sanitario, nella sua funzione di promotore di percorsi assistenziali (aziendali ed interaziendali) sicuri e centrati sul paziente, dovrà pertanto riconoscere in prima istanza la natura complessa della realtà in cui si trova ad agire, ${ }^{5}$ avvalendosi degli strumenti del Risk Management in un'ottica propositiva e favorendo la diffusione di una vera e propria cultura della sicurezza ${ }^{6}$ all'interno della propria Azienda mediante la diffusione di strumenti di identificazione e analisi del rischio. La sfida rappresentata dalla conversione degli ospedali in organizzazioni ad alta affidabilità (high reliability organizations) identifica, d'altronde, un processo critico che richiede un cambiamento culturale accompagnato da innovazioni tecnologiche ed organizzative coerenti, i cui effetti positivi risultano apprezzabili sul lungo termine. ${ }^{7} \mathrm{La}$ priorità dovrebbe essere quella di agire sugli atteggiamenti degli operatori sanitari, favorendo la rimozione delle barriere psicosociali nella comunità di pratica (in primis medici e sanitari) che rendono le strutture sanitarie non sufficientemente sicure.

Vincent e Amalberti (2016) nel loro recente volume Safer Healthcare ${ }^{8}$ riflettono sul ruolo della leadership in merito alla gestione del rischio, evidenziando come i principali limiti siano rappresentati dal riconoscimento stesso delle condizioni lavorative e dei livelli di affidabilità del sistema, spesso associati all'improvvisazione (per quanto la resilienza del sistema rappresenti un valore aggiunto rilevante) oppure a strategie di adattamento attivamente incoraggiate. Come sottolineato dagli autori, il linguaggio e la pratica del miglioramento della sicurezza risultano più familiari allo staff di prima linea, mentre a livello esecutivo prevalgono la logica del controllo e della mitigazione del danno. Risulterebbe fondamentale l'adozione di un linguaggio comune a tutti i livelli dell'organizzazione, ed un possibile approccio consisterebbe nell'analisi della condotta dei manager (a cominciare dalle figure del Direttore e Coordinatore di Unità Operativa), allo scopo di orientarli verso l'adozione di interventi espliciti e pianificati e di un insieme di strategie per la sicurezza. In tale ottica, i manager risulterebbero definibili come di prima linea proprio in virtù del notevole impatto che le loro decisioni assumerebbero in termini di sicurezza per il paziente.

Nel contesto delle Aziende Sanitarie, il Collegio di Direzione e i Dipartimenti rappresentano l'ambito organizzativo istituzionale in cui tali linee di governo si sviluppano e dove i principi, le metodiche e gli obiettivi ad esse correlati vengono valutati e condivisi fattivamente.

Il ruolo della componente manageriale si esplica inoltre nello sviluppo di un elevato livello di integrazione fra le varie componenti del sistema, a cui contribuiscono gli strumenti di uniformazione del linguaggio (es. documentazione clinica), la regolazione dei processi di lavoro condivisi, gli strumenti che strutturano la comunicazione (es. briefing), il presidio delle interfacce, il passaggio di consegne e $i$ trasferimenti tra $i$ diversi setting assistenziali, oltre che lo sviluppo di una cultura di reciproco rispetto tra le professioni. ${ }^{9}$

La valorizzazione del lavoro in team a tutti i livelli dovrebbe pertanto rappresentare uno degli obiettivi principali, se si considera che una carente modulazione delle risorse medico-infermieristiche è alla base di una quota considerevole di eventi avversi. In tale prospettiva assumono una particolare rilevanza anche l'insieme delle iniziative formative dedicate agli operatori, comprendenti anche lo sviluppo delle Non-Technical Skills, ${ }^{10}$ in qualità di strumento destinato a consolidare e ad accrescere le competenze dei professionisti.

Un ulteriore aspetto è quello legato alla prevenzione del contezioso e alla tutela dei professionisti operanti nelle strutture sanitarie, che al di là delle evidenti implicazioni di natura economica e medico-legale può comportare un disfacimento degli equilibri e del clima lavorativo, con notevoli ripercussioni in termini di sicurezza per il paziente. Le competenze derivanti da una formazione igienistica rappresentano indubbiamente un punto di forza nello sviluppo di una gestione consapevole del rischio, improntata alla conoscenza e alla valutazione dei processi organizzativi, anche in considerazione dell'expertise maturata in un'ampia gamma di settori (ad es. dalla prevenzione delle infezioni correlate all'assistenza alla valutazione delle tecnologie) direttamente correlati all'ambito della prevenzione del rischio mediante modalità siste- 
miche (ad es. multidisciplinari, trasversali, ecc.).

L'approccio di un Direttore Sanitario risulta pertanto orientato alla necessità di coniugare il perseguimento degli obiettivi aziendali alla costruzione di una solida cultura della sicurezza, basata sulla valorizzazione del lavoro in equipe e sulla formazione continua. Il fine ultimo è quello di andare oltre la semplice osservanza degli aspetti meramente procedurali oppure legati alle logiche di accreditamento (ad es. il recepimento delle Raccomandazioni Ministeriali e/o delle Buone pratiche per la sicurezza), per costruire una rete di collaborazioni che possa estendersi in maniera trasversale (anche al di fuori dei confini aziendali) allo scopo di garantire percorsi assistenziali sicuri ed efficaci.

Nel contesto della medicina interna, in cui oltre la metà degli errori diagnostici è dovuto a criticità di natura organizzativa, ${ }^{11}$ la necessità di favorire la comunicazione e la collaborazione interprofessionale rappresentano una evidente necessità, anche in virtù della crescente complessità assistenziale e dell'elevato grado di integrazione multidisciplinare richiesto.

L'ambito della Medicina interna risulta infatti sempre più caratterizzato dalla presa in carico di pazienti cronici complessi oggetto di ricoveri ripetuti spesso di durata limitata e tali da far emergere a livello internazionale l'opportunità di costituire sezioni dedicate a degenza breve (Acute Medical Units ${ }^{12}$ ) con presa in carico post-dimissione in day-service del paziente stesso, piuttosto che con degenza media superiore agli otto giorni, condizioni di fragilità e comorbilità che spesso espongono all'insorgenza di complicanze (ad es. sepsi) che rappresentano una rilevante minaccia per la sicurezza del paziente. In tale scenario proprio la figura del medico internista emerge come quella del professionista dotato delle competenze appropriate per un approccio sistemico alla complessità dei casi in un modello (purtroppo spesso ancora futuribile) di regia del percorso del paziente fragile polipatologico che appare, alla luce dell'epidemiologia, la vera sfida per il Servizio Sanitario nei prossimi anni. Il medico internista si pone inoltre come il naturale interlocutore con i setting delle cure intermedie e delle cure primarie, sedi di elezione per la gestione della cronicità, laddove i fattori comunicativi rappresentano un elemento imprescindibile (ed ancora ad oggi fragile a causa della frammentazione dei sottosistemi e dei modelli organizzativi) per la garanzia di una corretta presa in carico.

Una comunicazione di qualità e sostenuta da solide competenze comunicativo-relazionali rappresenta un pilastro fondamentale per la pratica professionale $e$ per l'erogazione di un'assistenza efficace, appro- priata, sicura ed efficiente, ${ }^{13}$ specie se associata ad un maggior coinvolgimento di pazienti e caregivers.

Il perseguimento di tali obiettivi è estendibile a tutti i settori dell'organizzazione e non può prescindere dal ruolo attivo della dirigenza medica e di tutti professionisti coinvolti nell'erogazione delle cure, destinati ad applicare tali principi nel contesto di una visione strategica della sicurezza coerente con gli obiettivi strategici della Direzione.

\section{Bibliografia}

1. Decreto Legislativo 30 dicembre 1992, n. 502. Riordino della disciplina in materia sanitaria, a norma dell'articolo 1 della legge 23 ottobre 1992, n. 421. GU n.305 del 30-12-1992 - Suppl. Ordinario n. 137.

2. Legge 8 marzo 2017, n. 24 -c.d. Legge Gelli-Bianco Disposizioni in materia di sicurezza delle cure e della persona assistita, nonché in materia di responsabilità professionale degli esercenti le professioni sanitarie.

3. Bizzarri G, Farina M. Strategia e gestione del rischio clinico nelle organizzazioni sanitarie: Approcci, modalità, strumenti e risultati. Milano: Franco Angeli; 2012.

4. Regione del Veneto. Deliberazione della Giunta Regionale n. 2255 del 30 dicembre 2016 - Semplificazione e riorganizzazione del modello organizzativo per la gestione della sicurezza del paziente e il contenimento del contenzioso nel Sistema Socio Sanitario del Veneto - approvazione del programma delle attività.

5. Ponzetti C, Appicciafuoco A, Benvenuto A, et al. Risk Management e sicurezza delle cure: Documento Programmatico dell'ANMDO. L'Ospedale gennaio marzo, 1, 2018.

6. Westrum R. A typology of organisational cultures. Qual Saf Health Care 2004;13:22e7.

7. Bagnara S, Parlangeli O, Tartaglia R. Are hospitals becoming high reliability organizations? Appl Ergon 2010;41:713-8.

8. Vincent C, Amalberti R. Safer Healthcare: Strategies for the Real World. 1st ed. Springer Open 2016, doi:10.1007/978-3-319-25559-0.

9. Tartaglia R, Vannucci A (Eds.). Prevenire gli eventi avversi nella pratica clinica. Berlin: Springer Verlag; 2013.

10. Gordon M, Fell CW, Box H, et al. Learning health 'safety' within non-technical skills interprofessional simulation education: a qualitative study. Med Educ Online 2017;22:1272838.

11. Graber ML, Franklin N, Gordon R. Diagnostic error in internal medicine. Arch Intern Med 2005;165:1493-9.

12. Reid LEM, Dinesen LC, Jones MC, et al. The effectiveness and variation of acute medical units: a systematic review. Int J Qual Health Care 2016;28:433-46.

13. Greco A, Greco M, D’Onofrio G, Sancarlo D. Il valore della comunicazione in medicina interna. Quad Ital J Med 2017;5(1):7-9. 


\title{
Il punto di vista di un Direttore di Medicina Interna
}

\author{
Stefano De Carli \\ S.O.C. $2^{\circ}$ Medicina Interna, P.O.U. S. Maria della Misericordia, ASUI Udine, Italia
}

Tradizionalmente e nell'immaginario collettivo, il medico ha da sempre impersonato il ruolo di colui che cura nel complesso processo assistenziale, dimenticandosi soventemente delle implicazioni organizzative. Questo ruolo organizzativo viene enfatizzato alla fine degli anni ' 70 dal processo di aziendalizzazione del Sistema Sanitario Nazionale. Il medico inquadrato nel Sistema Sanitario Nazionale diventa quindi elemento della gerarchia dell'organizzazione, cui si richiede la capacità di ragionare in termini aziendali, premesso che le aziende sanitarie si configurano come Enti pubblici senza fini di lucro. Diventa pertanto necessario acquisire quegli strumenti gestionali che pongono attenzione non solo agli aspetti di cura del paziente, ma anche alle ricadute economiche dell'attività sanitaria, agendo in funzione delle proprie responsabilità ai fini di sviluppare il processo aziendale. Pur mantenendo quindi la propria connotazione di protagonista del processo di cura, il medico deve comportarsi come il dirigente di una qualsiasi altra impresa che deve raggiungere i seguenti obbiettivi: i) massimizzare l'efficienza (il corrispettivo del profitto nelle aziende pubbliche e di quelle no profit in generale); ii) ridurre i costi di produzione del servizio sanitario; iii) mantenere la qualità del servizio offerto (e possibilmente di migliorarla).

Fino agli anni '70-'80 il modello di cura era incentrato e dipendeva esclusivamente dalle capacità del singolo professionista di trattare il paziente, basandosi sul bagaglio di esperienza maturato negli anni di studi e di professione. In quell'epoca le implicazioni economiche nel sistema sanitario erano considerate marginali e pertanto la componente manageriale svolgeva compiti prevalentemente di tipo

Corrispondente: Stefano De Carli, S.O.C. $2^{\circ}$ Medicina Interna, P.O.U. S. Maria della Misericordia, ASUI Udine, Italia. Tel.: +39.0432552602.

E-mail: stefano.decarli@asuiud.sanita.fvg.it

Articolo pubblicato secondo la Creative Commons Attribution NonCommercial 4.0 License (CC BY-NC 4.0).

${ }^{\circ}$ Copyright: the Author(s), 2019

Licensee PAGEPress, Italy

QUADERNI - Italian Journal of Medicine 2019; 7(6):49-52 amministrativo/contabile. In questo contesto va inquadrato qual è stato l'impatto dell'introduzione di nuove tecnologie (diagnostiche e terapeutiche) che non riducono $\mathrm{i}$ costi, ma generano un maggior assorbimento di risorse, richiedendo quindi un controllo della spesa. Di qui la necessità di introdurre modalità organizzative in grado di contenere (se non ridurre) i costi assicurando la miglior cura al singolo paziente paziente e l'accesso a cure di qualità a tutta la popolazione. La necessità di maggiore integrazione dei processi cura ha contribuito alla strutturazione dell'organizzazione sanitaria.

Donabedian, il padre della qualità dei sistemi sanitari aveva rilevato già negli anni ' 60 come la qualità dell'assistenza fosse correlata al rapporto tra effetti benefici e negativi, ma non fosse presente un rapporto di linearità tra risorse assorbite e risultati, evidenziando ancora una volta la necessità di una produzione efficiente di salute (i beni e servizi dell'azienda sanitaria). L'autore riteneva che anche la sicurezza delle procedure fosse una forma di efficientazione nella misura in cui il controllo degli errori riduceva il rischio di danni per la salute (contenendo in ultima analisi i costi). Questo processo assunse la denominazione di efficienza produttiva, poiché dipendeva da decisioni cliniche manageriali. Donabedian, anche se riteneva che i clinici non rivestissero un ruolo decisivo, rilevò che senza la loro partecipazione tali risultati non sarebbero stati possibili.

Con l'introduzione negli anni '90 della Clinical Governance il clinico trova gli strumenti per poter assumere un ruolo di cogestione con il manager nello sviluppo di un'organizzazione in grado di promuovere la qualità. Al clinico è richiesto di apprendere competenze della sfera manageriale e una parte della propria autonomia professionale viene sacrificata per assumere la responsabilità del proprio operato, che dev'essere misurabile e misurato. Si acquista la consapevolezza che ogni decisione clinica è anche di natura economica; viene coltivata la cultura dell'appropriatezza che è anche quella della sicurezza del paziente, poiché ogni azione superflua in medicina è potenzialmente dannosa (oltre che uno spreco di risorse); si valorizza la collaborazione con gli altri attori del sistema, poiché l'attuale complessità non 
permette quasi mai di poter gestire in solitudine il paziente, ma è necessario un approccio multidisciplinare.

Tuttavia l'attuale situazione del sistema sanitario soffre di un peculiare stato di dissociazione: mentre viene esasperata l'attenzione verso i migliori approcci diagnostici e terapeutici, vengono trascurati gli aspetti prettamente gestionali e organizzativi che non si apprendono né durante la formazione universitaria, né si viene sufficientemente sensibilizzati ad apprenderla una volta inquadrati come dirigenti del SSN, quasi questi costituissero un accessorio culturale. Un'evidente contraddizione è rappresentata dalla figura del Direttore di Struttura che da migliore clinico del reparto non è mai riuscito a porsi come l'organizzatore che risolve le criticità e pianifica lo sviluppo della propria unità operativa. Quello che gli si richiede oggi è di incarnare il ponte fra appropriatezza clinica e organizzativa. Infatti da un lato deve promuovere lo sviluppo, la diffusione e l'applicazione delle buone pratiche cliniche (basate sull'EBM), condizione necessaria, ma non sufficiente, poiché solo una parte delle problematiche in medicina sono affrontabili e risolvibili sulla base di evidenze scientifiche provate. D'altro canto deve fungere da raccordo fra la componente manageriale e decisionale del sistema sanitario e la parte clinica operativa dell'organizzazione. È pertanto sempre più necessario un Primario che sviluppi anche competenze di leadership. Con tale termine si intende la capacità di un individuo di aggregare il consenso di un gruppo e di condurlo ad azioni in grado di gestire il cambiamento. Essere leader significa essere autorevoli (non autoritari), per capacità che sono riconosciute in quanto guadagnate nella pratica quotidiana e non limitate alla posizione all'interno dell'organigramma o per anzianità di servizio. Il clinico per forma mentale focalizza il proprio ragionamento sul singolo caso tralasciando il contesto epidemiologico/demografico e molti altri aspetti propri dei processi di cura. L'acquisizione di strumenti manageriali al servizio dei percorsi sanitari, migliora la risposta senza dimenticare le ricadute in termini di umanizzazione delle cure, contestualizzazione sociale, carico economico della patologia e mantenimento/promozione dello stato di benessere. Un Direttore di Struttura non può essere un contabile ma deve saper tradurre i dati in informazioni, non deve essere un logico ma deve strutturare le proprie scelte in algoritmi operativi. Deve contestualizzare la programmazione sovra-aziendale in obiettivi condivisi, pianificando delle attività e valutando periodicamente lo stato di avanzamento, attraverso la gestione delle risorse disponibili (non solo spesa sanitaria, ma anche risorse di personale, professionali, ecc...). La giurisprudenza, a decorrere dall'art. 7 comma 3, DPR 27-3-1969 (Ordinamento interno dei servizi ospedalieri), prevede che il Primario risponda sia dell'operato tecnico-professionale, sia dei deficit organizzativi del reparto a lui affidato, costituendo la figura che dirige l'organizzazione della struttura, ne dispone l'assetto complessivo ed effettua la vigilanza sul personale sanitario, tecnico e di supporto. $\mathrm{Al}$ di là di questi compiti di responsabilità burocratica, il ruolo del Direttore di Struttura moderno è quello di favorire lo sviluppo del clima e della cultura organizzativa della propria struttura, agendo, come già detto da ponte con la componente manageriale e i decisori. Deve pertanto esplicitare e condividere con i propri collaboratori la mission e la vision aziendale, di dipartimento e di reparto. Deve contribuire a promuovere l'adozione di uno stile di comunicazione congruo, in relazione al diverso interlocutore (singolo paziente e associazioni, care giver, stakeholder, ecc...). Deve porre attenzione verso il clima interno per ottenere un'adeguata motivazione del personale. La cultura organizzativa è un insieme di valori non astrusi o astratti, ma è costituita da principi di fondo condivisi e diffusi in sintonia con la missione e la visione dell'organizzazione. La creazione e la diffusione di ogni cambiamento non è solo un impegno progettuale e di comunicazione, ma è un approccio operativo in cui lo stile di leadership rappresenta un modello. Il Direttore di Struttura deve cioè essere il primo a praticare una cultura volta alla qualità, alla costruzione di percorsi clinici, alle tematiche del rischio clinico e della prevenzione degli errori, individuando le criticità dell'organizzazione anche $\mathrm{e}$ soprattutto in modo proattivo. Deve promuovere una nuova cultura della sicurezza, dove l'errore non deve essere più visto come una colpa, ma come un'opportunità di crescita. Incoraggiando la segnalazione degli eventi avversi, organizza e coordina audit con tutti gli attori coinvolti nell'evento, perché questo strumento della Clinical Governance costituisce la funzione di monitoraggio per il mantenimento della qualità dell'assistenza, per un più appropriato impiego delle risorse e il contenimento del rischio clinico. Gli audit clinici dovrebbero svolgersi non solo in occasione di eventi avversi, ma dovrebbero essere effettuati periodicamente su tematiche individuate all'interno dell'organizzazione che abbiano una rilevanza strategica e necessitino di una revisione per migliorare i percorsi e i processi di cura.

L'evoluzione del sistema sanitario riconosce ogni medico come dirigente, quindi come un professionista che deve essere in grado di sviluppare capacità fino a ieri riservate ai Direttori di Unità Operativa. Tale passaggio, riconosciuto a livello normativo, deve diventare parte integrante della cultura del medico (non solo internista) favorendo lo sviluppo di capacità organizzative che migliorino il funzionamento delle 
strutture sanitarie. In un prossimo futuro è prevedibile che il progressivo diffondersi della cultura della Clinical Governance possa contribuire a risolvere i problemi della complessità delle organizzazioni sanitarie, dove i legami deboli prevalgono e quindi le leadership autoritarie e gerarchiche con il tradizionale modello organizzativo top-down sono destinate al fallimento. Tale assetto infatti fa percepire ai componenti dell'organizzazione, e al medico in particolare, la sensazione di limitarsi a essere un mero esecutore di decisioni che spesso non condivide. Il Dipartimento costituisce la sede dove possono essere sviluppati programmi di integrazione, di miglioramento della qualità e di promozione della Clinical Governance. Questo si tradurrebbe in un'auspicabile strutturazione bottom-up, in cui il management e il middle-management assumono il ruolo di supervisione e di coordinamento, mantenendo comunque la responsabilità del percorso strategico in sintonia con $\mathrm{i}$ programmi aziendali. Un Dipartimento così strutturato potrebbe costituire la strada per promuovere politiche di miglioramento continuo e benchmarking dei risultati; ottimizzazione di progetti di collaborazione interaziendali e di modelli di collaborazione a rete. L'organizzazione sarebbe in grado di dare risposta alle crescenti necessità di figure di case manager promosse sia dalla buona pratica clinica che dal modello sociale, di promuovere una cultura di presa in carico del paziente, rispondendo con servizi di qualità alle necessità di salute (polipatologie, invecchiamento della popolazione, ecc...) e alle aspettative dell'utenza e degli stake-holder. $^{1-4}$

\section{Bibliografia}

1. Davies HT, Harrison S. Trends in doctor-manager relationship. BMJ 2003;326:646-9.

2. Edwards N. Doctors and managers: poor relationships may be damaging patients - what can be done? Qual Saf Health Care 2003;12:i21-4.

3. Dobnabedian A. An introduction to Quality Assurance in Health Care. Oxford: Oxford Univeristy Press; 2003.

4. Zanetti M. Il medico e il management, II edizione. Accademia Nazionale di Medicina; 2007. 


\title{
La metà oscura del rischio clinico: i sinistri in medicina interna
}

\author{
Francesco Corradi \\ Medicina Interna ad alta Complessità Assistenziale 2, Azienda Ospedaliera Universitaria Careggi, Firenze, Italia
}

\section{Introduzione}

L'Italia ha registrato, nel corso degli ultimi 15 anni, un aumento preoccupante del numero di contenziosi in tema di responsabilità professionale sanitaria. La diversa percezione del diritto alla salute del cittadino, le crescenti aspettative di risultato, i cambiamenti occorsi alla giurisprudenza hanno contribuito ad aumentare il numero di denunce con importanti conseguenze sia per il sistema sanitario, per un aumento dei costi diretti (coperture assicurative) che indiretti (la medicina difensiva praticata in autotutela dal Medico per evitare i contenziosi), sia per il sistema assicurativo, con una netta riduzione del numero di imprese attive in questo settore e conseguente aumento dei costi delle coperture per il sanitario e per le Aziende. ${ }^{1}$ Il ricorso alla Medicina difensiva incide sulla spesa sanitaria per circa il $10,5 \%$, secondo i dati riportati dalla Commissione Parlamentare d'inchiesta sugli errori sanitari. Il Medico Internista, pur con alcune differenziazioni in base alla sede di lavoro (Pronto Soccorso, Medicina Interna, Ambulatorio) e/o alle mansioni svolte, talora comprensive anche di manovre invasive, in questi anni ha visto una sostanziale stabilità dei costi delle coperture. ${ }^{2}$

\section{Errori e sinistri: epidemiologia del contenzioso. Diamo i numeri!}

Prima di addentrarci nelle definizioni e nella tassonomia dell'errore e, più in generale della malpractice del Medico è utile, ai fini della comprensione delle

Corrispondente: Francesco Corradi, Medicina Interna ad alta Complessità Assistenziale 2, Azienda Ospedaliera Universitaria Careggi, Firenze, Italia.

E-mail: francescocorradi72@gmail.com

Articolo pubblicato secondo la Creative Commons Attribution NonCommercial 4.0 License (CC BY-NC 4.0).

${ }^{\circ}$ Copyright: the Author(s), 2019

Licensee PAGEPress, Italy

QUADERNI - Italian Journal of Medicine 2019; 7(6):53-60 dimensioni del problema, fornire alcuni dati che riguardano incidenza e prevalenza dei sinistri in area medica. In accordo al mandato ricevuto dall'intesa stato-regioni del 2008 e dal decreto ministeriale datato 11/12/2009 recante l'istituzione del sistema informativo SIMES (Sistema Informativo per il Monitoraggio degli Errori in Sanità), l'Agenzia Nazionale per i Servizi Sanitari Regionali (AGENAS) ha presentato il primo rapporto annuale sul monitoraggio delle denunce di sinistri. ${ }^{3}$ Lo scopo di Agenas è quello di effettuare un monitoraggio costante delle denunce e di produrre dati attendibili a livello nazionale sulla sinistrosità delle strutture pubbliche. I dati trasmessi provengono da 20 regioni e province autonome caratterizzate ciascuna da un proprio modello di rilevazione e gestione delle denunce di sinistri e da un peculiare modello di gestione del rischio. I dati, elaborati sulla base di 21 indicatori, rendono in questo modo una prima fotografia del contesto di riferimento del nostro Paese. Oggetto della rilevazione dell'Osservatorio Nazionale Sinistri sono le richieste di risarcimento pervenute alle strutture sanitarie pubbliche per eventi anche accaduti anni prima della pubblicazione dei dati, le denunce cautelative effettuate dalle strutture sanitarie nel 2014 e le notifiche di atti giudiziari civili e penali avanzate sempre nei confronti delle strutture sanitarie pubbliche. Sul tema va precisato, tuttavia, che solo il $14 \%$ delle denunce per sinistro avviene entro i primi mesi mentre la stima cresce fino al $70 \%$ a 4 anni. Da questo rapporto riportiamo i dati salienti utili ai fini della nostra disamina del problema iniziando dalla geografia del contenzioso: dal sud e dalle isole provengono il $44 \%$ circa dei casi, il $32 \%$ dal Nord, il $23 \%$ dalle regioni centrali con un indice medio di sinistrosità regionale, che definisce la frequenza con cui si verificano gli errori in sanità denunciati, pari a 20,94 su 10 mila casi (dimissioni). I procedimenti intraprendono una via stragiudiziale nel $74,44 \%$, giudiziale civile e penale rispettivamente nel 7,77 e nel 7,54\%. Il costo medio dei sinistri liquidati corrisponde a 52.368,95 Euro (284.669 per il decesso; 45.033 per lesioni personali) e la grande maggioranza dei sinistri denunciati, circa il $65,86 \%$, riguarda casi di lesioni personali, mentre i decessi rappresentano il 12,88. Il $17,31 \%$ dei sinistri è da riferire ad un errore diagnostico, il 6,54 alla terapia, il 33,55\% ad interventi, il 
$18,49 \%$ all'assistenza. Il 52,43\% avviene nell'ambito di un ricovero ordinario, il 15,48\% in pronto soccorso, il 2,33\% in ricovero day hospital, il 9,34\% durante un accesso ambulatoriale (Figura 1). Tra i dati più interessanti emerge la fascia d'età del presunto danneggiato a cui corrisponde il maggior numero di sinistri, pari al $21,29 \%$ delle pratiche che interessa la popolazione compresa tra i 65 e gli 80 anni. Per quanto riguarda, infine, la risoluzione dei sinistri occorre precisare che circa il $75 \%$ hanno seguito un iter stragiudiziale mentre solo nel $23 \%$ dei casi esso è costituito da procedimenti giudiziali. I dati dell'Associazione Nazionale delle Imprese Assicuratrici (ANIA) ${ }^{4}$ e quelli derivanti dallo studio sull'andamento del rischio da medical malpractice nella Sanità pubblica e privata fornito da Marsh \& McLennan, ${ }^{5}$ tuttavia, non sono del tutto sovrapponibili con i precedenti. In alcuni casi un apparente effetto di contrazione del numero di denunce potrebbe trarre in inganno poiché riflette, in realtà, la marcata tendenza delle strutture sanitarie a gestire in proprio (come ad esempio Liguria, Toscana, Puglia e Basilicata) o in auto-assicurazione i sinistri. In altri casi si utilizza un sistema misto, soprattutto per quelli di lieve entità, se non addirittura l'intero comparto della responsabilità civile medica. Ciò ha avuto come conseguenza quello di fare uscire i dati relativi ai sinistri dal perimetro statistico censito da ANIA. I dati di ANIA, riferiti fino al 2017, ${ }^{4}$ e quelli derivanti dalla nona edizione dello studio sull'andamento del rischio da medical malpractice nella Sanità pubblica e privata fornito da Marsh \& McLennan riferita ai sinistri denunciati nell'intervallo temporale 2004-2016 aggiornati nel corso del $2017 \mathrm{su}$ un campione di 42 strutture ubicate nelle regioni Lombardia, Emilia Romagna, Toscana, Veneto, Sardegna, Valle d'Aosta, Trentino Alto Adige, Liguria, Marche, Sicilia, rivelano 10.452 sinistri nell'intervallo temporale di riferimento con una media annua di $804 \mathrm{su}$ 964.000 ricoveri, 27.848 posti letto, su 18.244 medici e 44.429 infermieri. ${ }^{5}$ Una media dei sinistri annuali pari a 27,5/anno per struttura (Figura 2). Nell' area medica, che comprende, oltre alla Medicina Interna, anche la Cardiologia/UTIC, l'Oncologia, la Gastroenterologia e Endoscopia Digestiva, la Neurologia, la Medicina Trasfusionale, la Pneumologia e Fisiopatologia Respiratoria, la Dermatologia, la Nefrologia/dialisi, la Geriatria, l'Infettivologia, l'Ematologia, la Diabetologia, l'Endocrinologia, la Lungodegenza, le Cure Palliative, l'Allergologia e la Medicina Sportiva, la frequenza di eventi avversi registrata è pari al
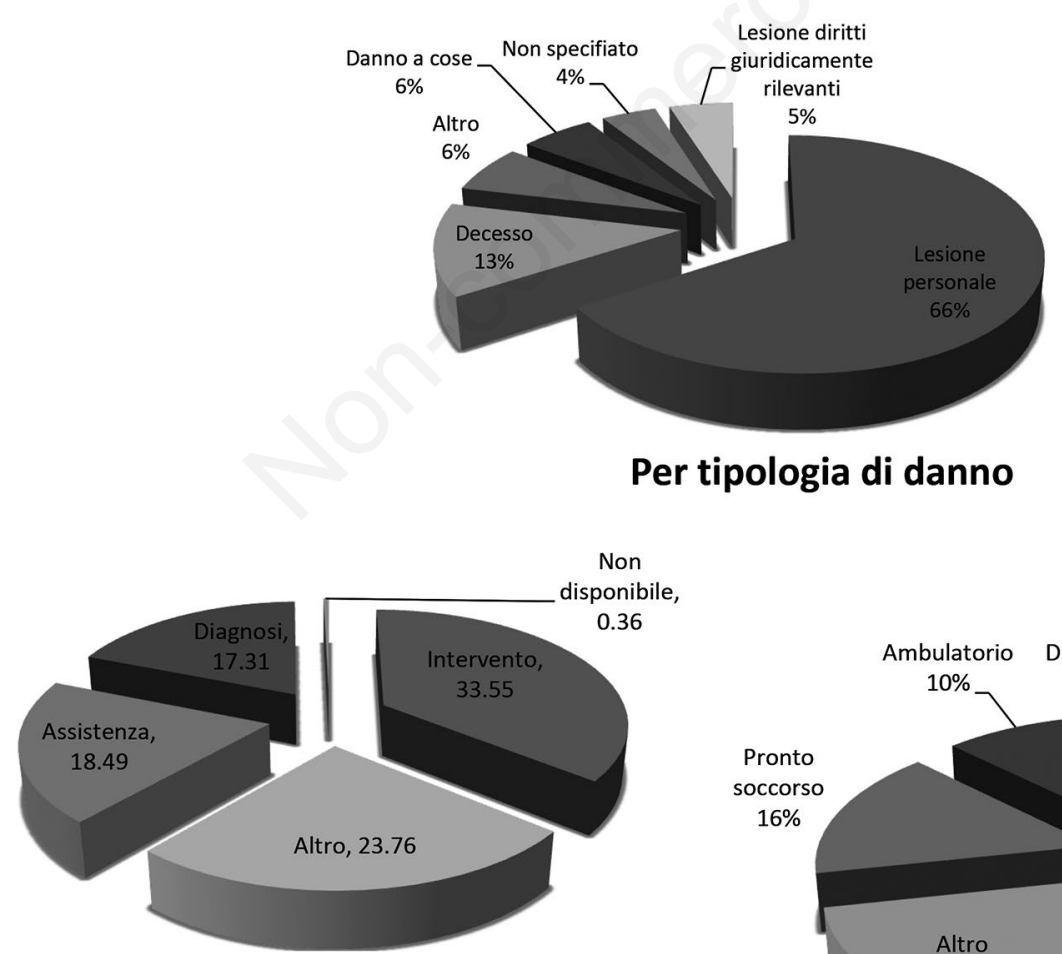

Per tipo di prestazione

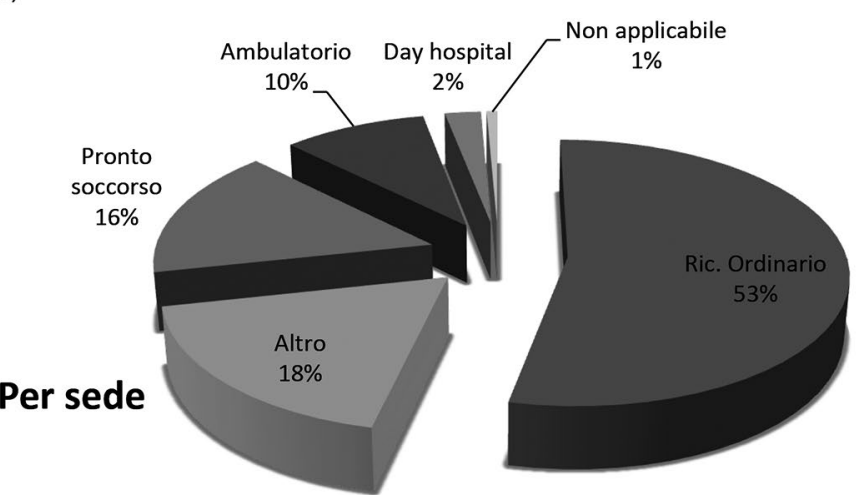

Figura 1. Percentuali di sinistri aperti nel 2014. Fonte: Agenzia Nazionale per I Servizi Sanitari Regionali (age.na.s). Monitoraggio delle denunce di sinistri 2014. Rapporto annuale Ottobre 2015. 
$12,1 \%$, di poco superiore a quello dell'area emergenzaurgenza (che però comprende solo il Pronto Soccorso, la Chirurgia d'Urgenza, la Medicina d'Urgenza ed il Servizio Territoriale di Emergenza) ma di gran lunga inferiore a quella chirurgica che rappresenta, con il suo $45,1 \%$, quella maggiormente esposta, seguita dalla Materno-infantile. ${ }^{6}$ Tra i sinistri maggiormente rappresentati, fatta eccezione per il chirurgico, che pesa per il 34,9\%, sono rappresentati quelli diagnostici $(18,5 \%)$, le cadute accidentali $(10,3 \%)$, gli errori terapeutici $(9,4 \%)$, le infezioni $(5,9 \%)$ e gli errori correlati a pro- cedure invasive $(4,4 \%) .{ }^{7}$ Queste sei tipologie di errore a cui, fatta eccezione per il chirurgico, è esposto, al pari di altre specialità, anche il Medico Internista, costituiscono più dell' $80 \%$ del totale del campione (Figura 3). Dal punto di vista strettamente economico il costo totale dell'intero database sinistri è pari a 921 milioni di euro di cui il $49 \%$ liquidato con un costo medio di 88.198 euro; l'impatto economico maggiore è attribuito, prevedibilmente, agli errori chirurgici (27\%) mentre gli errori diagnostici e quelli terapeutici pesano per il 14\% circa rispettivamente. Quando il

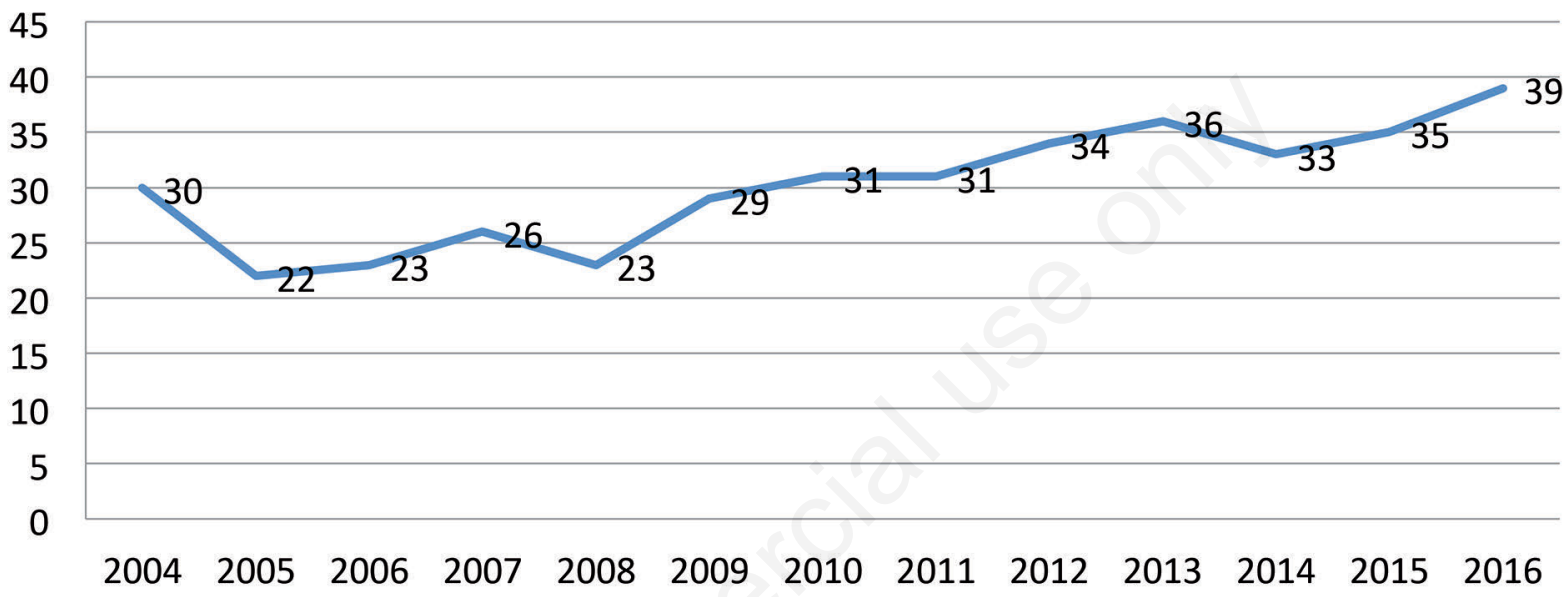

Figura 2. Media annua del numero dei sinistri per anno di denuncia. Fonte: Report MEDMAL-sintesi Studio sull'andamento del rischio da medical malpractice nella sanità italiana pubblica e privata. $9^{\circ}$ Edizione 2018.

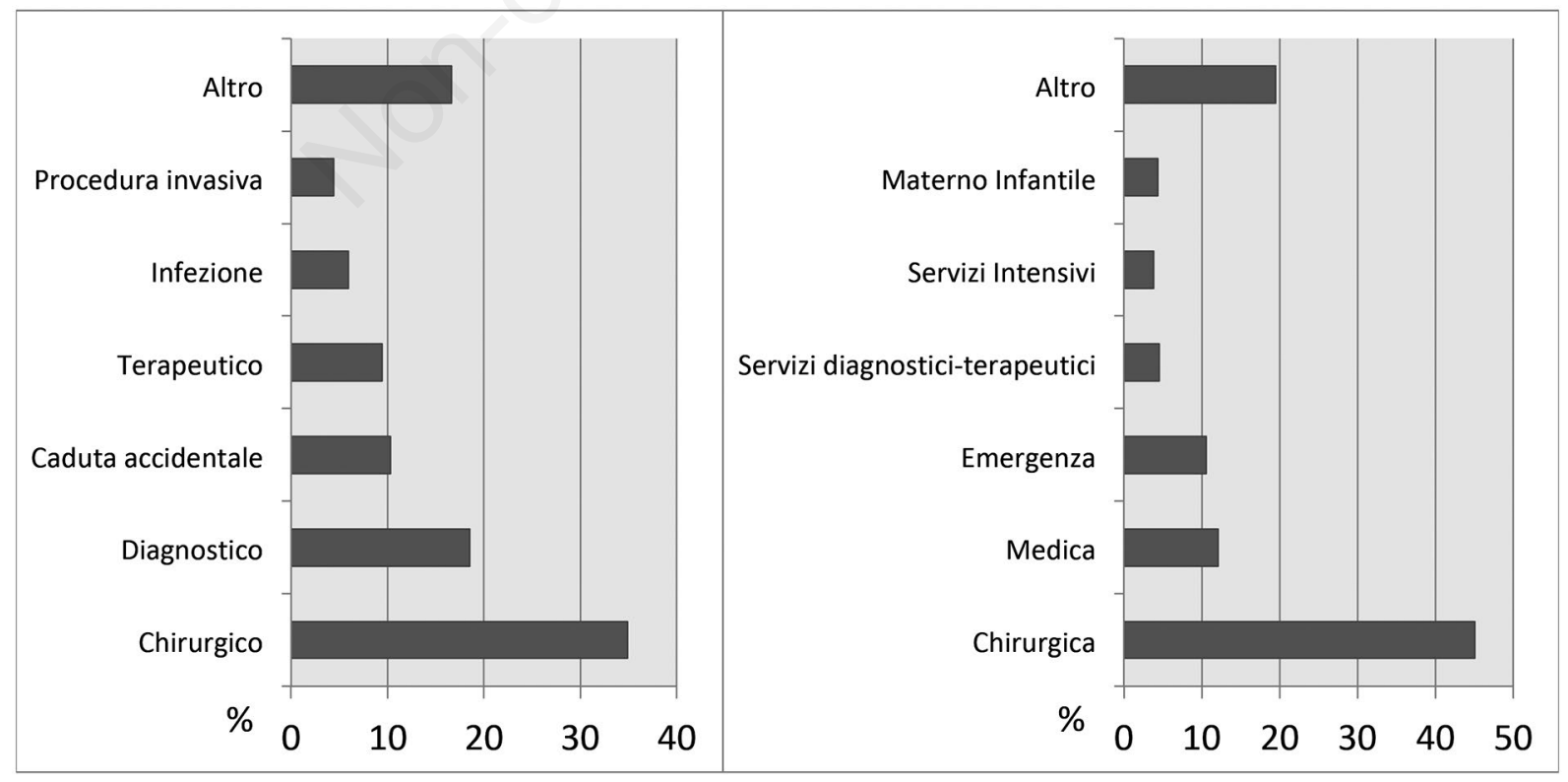

Figura 3. Analisi delle frequenza: aree a rischio e tipologia di evento. Fonte: Report MEDMAL-sintesi Studio sull'andamento del rischio da medical malpractice nella sanità italiana pubblica e privata. $9^{\circ}$ Edizione 2018. 
costo di una denuncia è pari o supera i 500.000 euro il sinistro viene considerato un top claim; essi impattano per il 4,1\% degli eventi totali ma assorbono, in termini economici, il $42 \%$ del costo totale con un $67,6 \%$ che si colloca fra i 500.000 e il 1.000 .000 di euro. ${ }^{8}$ Sono gli errori che esitano nel modo peggiore, con una percentuale di decessi pari al $42 \%$. Nei top claims ricorrono, oltre agli errori chirurgici $(23,2 \%)$ e da parto (21\%), anche i diagnostici, ovvero errori ai quali è esposto anche il Medico Internista, tutt'altro che infrequenti $(20 \%)$, quelli terapeutici $(13,4 \%)$ e le infezioni $(6,3 \%)$. La percentuale dei procedimenti giudiziari, sia in ambito civile che penale, risulta più elevata per i top claims (27 e 14\% rispettivamente) rispetto al campione generale che si attesta, rispettivamente sul 18 e 5\%. A tale proposito appare utile fornire alcune informazioni statistiche sulla giurisprudenza penale in tema di responsabilità medica. A titolo di esempio, da una ricerca condotta sulla giurisprudenza della quarta sezione penale della Corte di Cassazione nel triennio 2013-2015, emergono 67 sentenze pronunziate e depositate nel 2013, 56 nel 2014, 55 nel 2015. ${ }^{9}$ Sono numeri che dimostrano l'esiguità del numero di denunce presentate in ambito penale, specie se rapportate ad un popolo di professionisti del settore sanitario pari a circa 300.000 unità. Di gran lunga superiore è, tuttavia, il numero dei casi per i quali sono avviate indagini preliminari per lesioni colpose o omicidio colposo ma ciò appare fisiologico poiché, proprio nella fase iniziale delle indagini, ancor prima che sia valutata la natura colposa delle condotte e la loro rilevanza causale nel determinismo del danno, non è possibile individuare subito a chi siano riferibili i trattamenti disposti in ambito sanitario. Le indagini preliminari, inoltre, unitamente all'udienza preliminare e ai primi due gradi di giudizio operano una funzione di selezione/filtro spiegando questi piccoli numeri. ${ }^{10} \mathrm{~A}$ fronte, tuttavia, di un $95 \%$ dei procedimenti penali per lesioni personali colpose che si conclude con un proscioglimento (in sede civile circa il 34\%) ciò che preoccupa il Sanitario e contribuisce in modo sensibile all'autodifesa applicata mediante la medicina difensiva sono la richiesta del risarcimento, il discredito che ne deriva e i tempi dei procedimenti che, mediamente, in Italia, per una causa, si attestano, per aprirla, su un numero di giorni pari a 872,53 giorni e, per chiuderla, su 542,45 giorni $(7,87 \%$ di sinistri chiusi in via stragiudiziale entro l'anno). ${ }^{11}$

\section{L'errore medico: proviamo a definirlo}

La guida all'Esercizio Professionale caratterizza l'errore in questo modo: l'errore clinico si verifica quando, essendo presenti, manifesti e non equivoci $i$ sintomi fondamentali, un caso non sia correttamente inquadrato a causa di negligenza, imprudenza o imperizia dal medico nell'ambito di una delle malattie attualmente note alla scienza medica.$^{12}$ Qual è la validità della scienza medica e cosa intendiamo per malattie attualmente note? Esistono sintomi non equivoci? La medicina interna si prefigge l'obiettivo, non di conoscere una malattia, ma di ri-conoscere una certa malattia in un certo paziente. La malattia tuttavia è una costruzione mentale elaborata da studiosi che in un particolare momento storico, osservando fenomeni noti come sintomi e segni, la definiscono e la codificano nella Linea Guida corrispondente, accettata dalla comunità scientifica internazionale suggerendone il corretto percorso diagnostico e terapeutico. ${ }^{13}$ Poiché l'errore è un discostamento dall'idea di verità e, nello specifico, di verità scientifica, che, nella valutazione Medico Legale e, ancor più con l'introduzione della legge Gelli Bianco, deve fare riferimento a quella ritrovabile nelle linee guida di riferimento della patologia oggetto dell'errore evitabile, appare evidente che chi esamina l'operato del clinico e giudica dovrà aver sempre presenti, non solo la linea guida, ma anche la sua relatività temporale e, quindi, la storicità del giudizio clinico. Ancor più difficile, quindi, appare definire l'errore in Medicina Interna laddove le caratteristiche e la mission della specialità sono particolarmente complesse e varie spaziando da una difficile diagnostica differenziale alla gestione di multimorbilità nello stesso paziente, alla necessità di mantenere sempre una visione olistica, alle difficili scelte terapeutiche, alla scelta tra indicazioni e controindicazioni di una manovra diagnostica e/o terapeutica. E' quindi evidente che l'Internista, per le caratteristiche proprie della specialità, è esposto sia all'errore cognitivo che all'errore operativo. Il primo corrisponde all'errore diagnostico nel quale si dichiara che il paziente $A$ è affetto dalla malattia $M$ e coinvolge l'analisi decisionale. ${ }^{14}$ L'errore operativo è, invece, quasi sempre correlato alla terapia. Non sempre l'errore dell'Internista consiste in un difetto di osservazione (che rappresenta comunque una fase necessaria e indispensabile per porre una diagnosi corretta) ma può coinvolgere anche quella successiva ovvero quella del ragionamento. ${ }^{15}$ Gli errori che coinvolgono la capacità di classificare una malattia, comprenderne i fenomeni e predirne la progressione sono, chiaramente, di tipo cognitivo, mentre quelli che coinvolgono la capacità di modificarne la progressione identificano un errore operativo. ${ }^{16}$ L'errore medico può essere, inoltre, omissivo (mancata diagnosi, mancato trattamento) o commissivo (intervento errato, errori nel corso di una procedura invasiva); ad entrambi può conseguire un evento avverso clinicamente significativo. L'errore medico, in ogni caso, viene considerato come l'evento conclusivo di una catena di fattori nella quale il contributo dell'individuo che ha effettivamente commesso l'errore è l'anello finale ma non necessariamente il maggiore responsabile. 


\section{Errore e sinistro: non sono sinonimi}

Quando valutiamo la malpractice si rende necessario stabilire se riteniamo errore solo ciò che conduce ad un danno oppure tutto ciò che si discosta dalla verità scientifica, anche se di fatto non ha prodotto alcun danno. In quest'ultimo caso, infatti, l'errore non si traduce in sinistro. ${ }^{17}$ Mentre per valutare il numero di sinistri basta contare le denunce, molto più complesso è fare una stima sufficientemente precisa del numero di errori; uno dei maggiori ostacoli è rappresentato, infatti, dal timore che il proprio errore possa generare conseguenze amministrative e/o medico legali, spingendo, il committente a negare o, comunque, a non comunicarlo. In USA, secondo il rapporto dell'Institute of Medicine, risalente al lontano 1999 dal titolo to err is human, ogni anno muoiono negli ospedali americani, tra i 44.000 e i 98.000 paziente per malpractice con una spesa per errori e danni causati pari a circa 37,6 miliardi di dollari. ${ }^{18}$ Queste cifre, assai preoccupanti, sono attendibili? Le stime disponibili, basate su studi retrospettivi e prospettici condotti su campioni di cartelle cliniche, sono viziate da bias per difetto (molti errori non vengono svelati) o per eccesso (eventi giudicati evitabili non erano tali). Questi studi, inoltre, si concentrano su eventi avversi conseguenti ad errori terapeutici più semplici da intercettare e valutare diversamente da un errore diagnostico, molto più difficile da contare ed evidenziare in termini di danno conseguente (molto spesso un errore diagnostico produce un danno in modo indiretto). ${ }^{17} \mathrm{La}$ maggior parte di informazioni sulla frequenza degli errori diagnostici (e con il medesimo procedimento essi sono valutati in sede medico-legale e peritale) provengono da studi di confronto clinico-autoptici e, di conseguenza, applicabili ad eventi e/o patologia potenzialmente fatali. ${ }^{19} \mathrm{Il}$ riscontro autoptico, in effetti, rivela la presenza di discrepanze macroscopiche nel $10-20 \%$ dei casi ma è sempre meno utilizzato (in meno del $10 \%$ dei casi in USA) sebbene rimanga il gold standard per fornire dati utili ad incrementare l'accuratezza diagnostica. Non sempre, tuttavia, i dati anatomo-patologici sono sufficienti a porre una diagnosi certa o, comunque, a rivelare, con una probabilità prossima alla certezza, una discrepanza tale da porre in nesso causale un danno ad un errore diagnostico; inoltre, non infrequentemente, l'analisi autoptica può svelare un vasto numero di reperti incidentali che non hanno un valore causale o concausale, clinicamente rilevante, in relazione al decesso del paziente, ma restano, anzi, elementi confondenti nell'ambito del contenzioso. Ne deriva che è ancor più difficile reperire informazioni su errori diagnostici che non hanno condotto ad un evento morte ma solo ad un danno alla persona. D'altra parte, i dati disponibili confermano che gli errori diagnosti non sono meno frequenti di quelli terapeutici anche se è poi la decisione terapeutica conseguente proprio all'errore diagnostico la causa finale dell'evento avverso. Un noto Psicologo Cognitivo, Arthur Elstein che si è occupato in modo particolare di analisi decisionale nel pensiero medico, concludeva che un errore diagnostico viene compiuto in circa il $10-15 \%$ dei casi analizzati. ${ }^{20}$ Studi condotti negli Stati Uniti e in Europa, tra il 1991 e il 2004, confermano stime riguardo alla frequenza di eventi avversi variabile fra il $6,9 \%$ e il $17 \%$, confermando il dato di Elstein. Inoltre, quando confrontati con gli errori terapeutici, quelli diagnostici si rivelavano i peggiori in termini di entità del danno procurato ed i meno prevenibili. Non necessariamente l'errore diagnostico ed il sinistro conseguente riguardano patologie di particolare difficoltà interpretativa. In effetti la letteratura dimostra come le condizioni più frequenti ove si possono verificare errori di questo tipo sono le polmoniti $(6,7 \%)$, lo scompenso cardiaco (5,7\%), l'embolia polmonare $(4,8 \%)$, lo stroke $(2,6 \%)$ l'insufficienza renale acuta $(5,3 \%)$, il cancro della mammella, del colon retto, del polmone $(5,3 \%)$ e le infezioni delle basse e alte vie urinarie $(4,8 \%) .{ }^{21}$ La principale critica che potremmo avanzare agli studi e alle stime conseguenti sull'errore diagnostico che, come abbiamo visto, si attestano su percentuali variabili fra il 13 e il $15 \%$, deriva dal fatto che applicare il metodo scientifico e standardizzare i parametri di valutazione permette di valutare, in ogni caso, solo un numero ristretto di condizioni che si verificano nella pratica clinica ma, come sappiamo bene, il lavoro dell'internista (e l'accuratezza diagnostica), appare particolarmente complesso da giudicare dato l'alto numero di variabili da considerare nell'analisi decisionale che conduce ad una diagnosi influenzata da molti parametri tra cui quelli relativi al paziente che presenta molteplici comorbilità e complicanze contestuali che discostano il caso in oggetto da quello standard, preso come riferimento per la valutazione della eventuale mancata diagnosi o errato trattamento. ${ }^{19,22,23} \mathrm{Il}$ rischio di errore nella Medicina Interna è insito nella mission stessa della nostra specialità. A ciò si aggiunga che una diagnosi internistica richiede talora di esercitare tale analisi decisionale con accuratezza diagnostica in tempi relativamente brevi e in condizioni di particolare stress lavorativo come si può verificare nelle Unità Operative di Sub-intensiva Internistica o nei Dipartimenti di Emergenza-Urgenza.

\section{Tassonomia dell'errore cognitivo diagnostico}

È possibile identificare l'eziologia dell'errore diagnostico e formularne un inquadramento tassonomico? ${ }^{24}$ In letteratura vengono riconosciute tre categorie essenziali, gli errori senza colpa ovvero presentazioni cliniche insolite o mascherate, paziente 
non collaborante o non recettivo, gli errori del sistema tra cui si ricordano gli errori tecnici e i problemi correlati all'equipaggiamento e, infine, gli errori cognitivi tra cui annoveriamo difetti culturali, di raccolta dei dati e incapacità di sintesi. Nel 46\% dei casi l'errore diagnostico riconosce una causa duplice, di sistema e cognitivo. Meno comuni sono errori esclusivamente di sistema (19\%) o solo cognitivi (28\%), molto rari i primi ovvero gli errori senza colpa (Figura 4). La frequenza relativa degli uni o degli altri dipende dalla sorgente dei dati raccolti e dal tipo di errore. Ad esempio, i casi rilevati nei report delle Assicurazioni mostrano una prevalenza simile ( $72 \%$ correlati al sistema, $65 \%$ di tipo cognitivo); tuttavia, se consideriamo dati derivanti da riscontri autoptici rileviamo che il peso del fattore cognitivo è pari a circa il $90 \%$. Inoltre, mentre il ritardo diagnostico è più frequentemente imputabile ad un errore nel sistema $(89 \%)$, l'errata diagnosi riconosce per lo più un errore cognitivo $(92 \%) .{ }^{25}$

\section{Evoluzione della giurisprudenza del contenzioso sanitario. Legge Gelli-Bianco: facciamo un bilancio?}

L'andamento dei sinistri denunciati alle compagnie assicurative e contenuto nell'analisi del periodo 20102016 nel documento dell'Associazione Italiana delle Imprese Assicuratrici) denominato ANIA trends rappresenta uno strumento indispensabile per misurare gli effetti della nuova legge di riforma della responsa- bilità medica meglio nota come riforma Gelli approvata nella primavera del 2017 dal Parlamento. ${ }^{26} \mathrm{La}$ nuova normativa contiene novità importanti per prevenire gli errori connessi all'attività dei Professionisti e delle strutture sanitarie, incrementare gli standard di qualità delle prestazioni ma, allo stesso tempo, cercare di ridurre le incertezze entro le quali si volge l'attività sanitaria disincentivando il ricorso alla cosiddetta medicina difensiva.

Al trend negativo degli anni antecedenti il 2012 si sono opposte, o quantomeno hanno tentato di porre rimedio, prima il decreto Balduzzi nel 2012 e, successivamente la legge Gelli-Bianco nel 2017. ${ }^{27} \mathrm{Il}$ decreto Balduzzi, convertito con modifiche dalla legge $189 / 2012,{ }^{28}$ ferma restando la carenza di una normativa organica che disciplinasse, in maniera esauriente, la responsabilità professionale sanitaria, ebbe, senz'altro, un impatto innovativo, tentando di circoscrivere le ipotesi di reato alla sola colpa grave del sanitario, dando valore all'osservanza di linee guida e alle buone pratiche cliniche accreditate dalla comunità scientifica. ${ }^{29}$ In quel contesto e in quegli anni, tuttavia, la Giurisprudenza, pur ammettendo il valore della linea guida nell'orientare in modo appropriato le decisioni terapeutiche del sanitario sottraendole al soggettivismo, obiettava che le direttive in esse contenute non erano in grado di offrire standard legali precostituiti a causa della loro varietà e il diverso grado di qualificazione Proprio su questo punto il successivo intervento del parlamento con la riforma Gelli del $2017,{ }^{30}$ oltre a normare in modo organico la responsabilità professionale sanitaria, ha definitivamente cercato di standardizzare il ricorso alle Linee

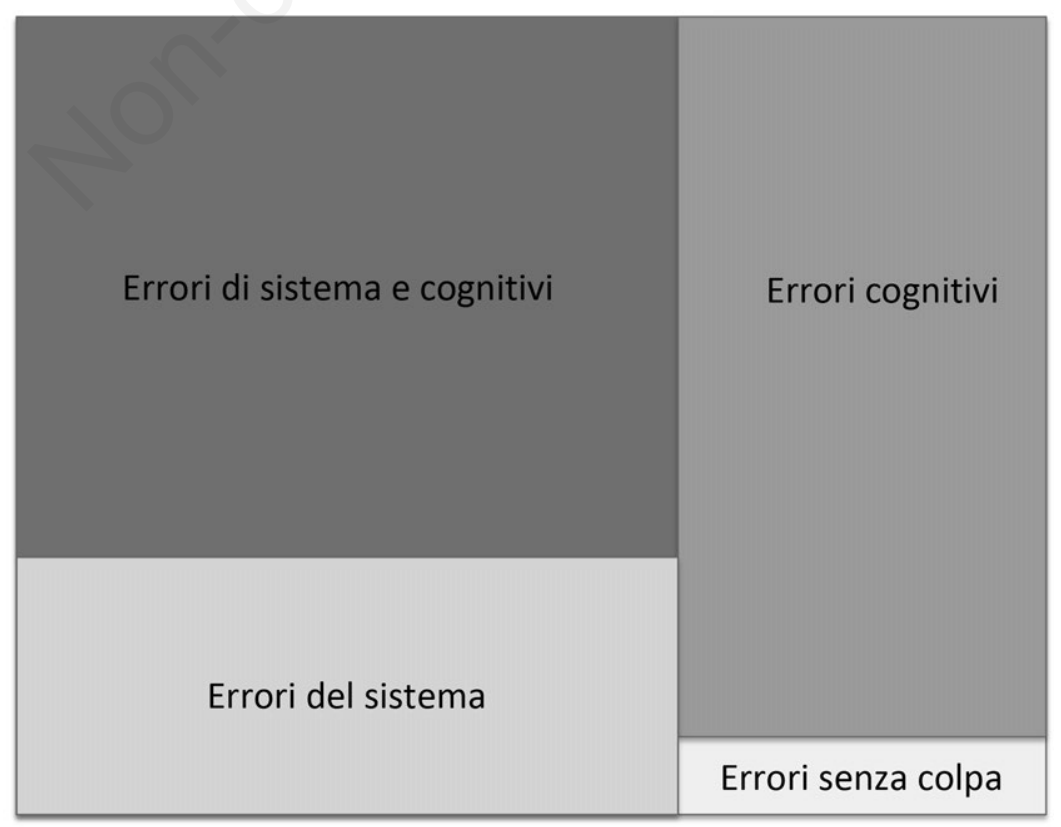

Figura 4. Rappresentazione schematica delle categorie di fattori favorenti l'errore diagnostico. 
Guida individuando e certificando le società scientifiche promulgatrici delle linee guida al fine di rendere la valutazione peritale la meno soggettiva possibile. È possibile fare un bilancio seppure parziale degli effetti di questa legge? A più di 2 anni dall'entrata in vigore della legge la situazione dei Medici nei tribunali resta sostanzialmente immutata, così come invariato è il numero di denunce che, per fare un esempio, nella sola Milano corrispondono a circa 300 nel solo anno 2017, circa uno al giorno di cui il $30 \%$ archiviate. Del restante $70 \%$ che procede in giudizio, circa un caso su cinque, vede una condanna per il Sanitario. Ciò che rimane inapplicato, nonostante siano trascorsi molti mesi, è la mancanza di quelle linee guida che dovevano rappresentare la cartina di tornasole per valutare l'operato dei medici che al momento resta, come prima, oggetto di analisi da parte di consulenti tecnici che continuano, pertanto, a svolgere nei processi un ruolo fondamentale basando le proprie valutazioni sulle linee guida internazionali e nazionali disponibili, accreditate a livello scientifico ma non a livello ministeriale. Pur essendo ancora prematuro trarre conclusioni definitive dati i tempi della giustizia, pur tuttavia, è ancora carente il pilastro della legge ovvero l'individuazione di linee guida in grado di tipizzare le condotte corrette, vincolando il Giudice nella sua decisione a modelli predeterminati con impatto favorevole, in ultimo, anche sulla medicina difensiva. In Italia delle numerose società scientifiche, al novembre del 2018, il Ministero della Salute risulta averne accreditate 293. Alla fase di accreditamento farà seguito l'intervento dell'Istituto Superiore di Sanità per definire gli standard per l'elaborazione delle raccomandazioni e realizzare una piattaforma informatica che le società potranno usare per presentare le loro proposte. ${ }^{31}$

\section{Conclusioni}

L'errore non necessariamente produce un danno poiché se dall'analisi del nesso di causa non emerge una correlazione tra errore e danno, non vi è colpa. Ciò significa che, di fatto, l'errore derivante dalla malpractice può non tradursi in sinistro. È compito dell'Unità di Rischio Clinico delle Aziende Sanitarie operare affinché si riduca la possibilità di compiere errori. E' compito della giurisprudenza far si che le cause per responsabilità professionale si svolgano con tempi e modi adeguati specie per ciò che riguarda 1'applicazione della Legge Gelli Bianco, riforma che ha normato efficacemente la responsabilità professionale e ha fornito tutti gli strumenti, se applicati correttamente e tempestivamente, per ridurre il riscorso allo strumento penale nei confronti del medico e per standardizzare il ricorso a linee guida certificate nazionali al fine di ridurre l'estrema variabilità e il soggettivismo della valutazione peritale. Oltre alla nuova normativa, i cui obiettivi comprendono anche quello di ridurre il ricorso alla medicina Difensiva, è auspicabile stimolare il ricorso, specie in ambito internistico, ad altre metodiche quali la cura degli aspetti relazionali, la scelta informata, il processo decisionale condiviso, la slow medicine, il nuovo orientamento della choosing wisely e la Medicina Narrativa, metodiche che se correttamente impiegate e sviluppate potranno fornire un importante contributo nel recuperare il rapporto medico-paziente ed il percorso diagnostico-terapeutico.

\section{Bibliografia}

1. Agenzia Sanitaria Regionale dell'Emilia Romagna. La gestione del contenzioso nelle organizzazioni sanitarie. L'informazione in Pillole 2007;39.

2. Marcon G. Gestione del rischio clinico. Care 2003; 4:14-8.

3. Agenzia Nazionale per i Servizi Regionali Sanitari (age.na.s.). Monitoraggio delle denunce di sinistri 2015. Rapporto annuale 2015.

4. L'Assicurazione Italiana 2016-2017. Dossier Associazione Nazionale fra le imprese assicuratrici; 2017.

5. Marsh e McLennan. Studio sull'andamento del rischio da medical malpractice nella sanità italiana pubblica e privata. Report Medmal-Sintesi $9^{\circ}$ Ed. 2018.

6. Cohen D, Klyve-Wood M, Chan S. Trends in Malpractice Claims on Emergency Physicians: Timea nd Money. Ann Emerg Med 2014;64:4.

7. Cohen D, Chan SB, Dorfman M. Malpractice Claims on Emergency Physicians: Time and Money. J Emerg Med 2012;42:22-7.

8. Nauss M, Mucha J, Schultz L. Emergency Medicine Malpractice Claims: One Medical Group's Experience. Ann Emerg Med 2015;66:4.

9. Brusco C. Informazioni statistiche sulla giurisprudenza penale di legittimità in tema di responsabilità medica. Diritto penale contemporaneo 14 luglio 2016.

10. Piras O. La riforma della colpa medica nell'approvanda legge Gelli-Bianco. Diritto penale contemporaneo 25 marzo 2016.

11. Cupelli C. La colpa lieve del medico tra imperizia, imprudenza e negligenza: il passo avanti della Cassazione (e i rischi della riforma alle porte). Diritto penale contemporaneo 27 giugno 2016.

12. Guida all'esercizio professionale per i Medici Chirurghi e Odontoiatri. Con il patrocinio di FNOMCeO (Federazione Nazionale degli Ordini dei Medici Chirurghi e Odontoiatri). Edizioni Medico Scientifiche; 2006.

13. Federspil G, Vettor R. Rational error in internal medicine. Inter Emerg Med 2008;3:25-31.

14. Graber ML, Franklin N, Gordon R. Diagnostic error in internal medicine. Arch Intern Med 2005;165:1493-9.

15. Crupi V, Gensini GF, Motterlini M. La dimensione cognitive dell'errore in medicina. Milano: Franco Angeli; 2006.

16. Croskerry P. The importance of cognitive errors in diagnosis and strategies to minimize them. Acad Med 2003;78:775-80.

17. L'errore in Medicina. Editoriale Bollettino d'informazione sui farmaci. Ministero della salute. Direzione 
Generale della Valutazione dei Medicinali e la Farmacovigilanza. N.3 Maggio-Giugno 2001.

18. Kohn L, Corrigan J, Donaldsom M. To err is human. Building a safer health system. Washington, DC: National Academy Press; 2001. pp 509-513.

19. Graber ML. The incidence of diagnostic error in medicine. BMJ Qual Saf 2013;22:ii21-7.

20. Elstein A. Clinical reasoning in medicine. In: Higgs J, ed. Clinical reasoning in the health professions. Oxford, England: Butterworth-Heinemann Ltd.: 1995. pp 49-59.

21. Schiff GD, Hasan O, Kim S, et al. Diagnostic error in medicine. Analysis of 583 physician-reported errors. Arch Int Med 2009;169:1881-7.

22. Berner Eta S, Graber ML. Overconfidence as a cause of diagnostic error in medicine. Am J Med 2008;121:S2-S23.

23. Singh H, Schiff G, Graber ML, et al. The global burden of diagnostic errors in primary care. BMJ Qual Saf 2017;26:484-94.

24. Graber ML, Franklin N, Gordon R. Diagnostic error in internal meedicine. Arch Int Med 2005;165:1493-9.

25. Van Den Berge K, Mamede S. Cognitive diagnostic error in internal medicine. Eur J Int Med 2013;24:525-9.

26. Associazione Nazionale fra le Imprese Assicuratrici (ANIA). Trends. FOCUS RC Sanitaria. Novembre 2017.

27. Decreto Legge 3 settembre 2012, n. 158 GU Serie Generale n. 214 del 13/09/2012.

28. Legge 8 novembre 2012, n. 189. GU Serie Generale n. 263 del 10/11/2012 S. O. n. 201.

29. De Palma A. La medicina difensiva e il contenzioso nella legislazione nazionalein Medicina Difensiva: diffusione e impatto economico. Un modello di valutazione. Age.na.s. Monitor. Supplemento I quaderni 2015.

30. Legge 8 marzo 2017, n. 24. GU Serie Generale n.64 del 17-03-2017.

31. Le Pera A. Nella testa di chi processa i medici. Il Giornale della Previdenza dei Medici e Odontoiatri. Anno XXIII n. 5/2018. 


\title{
La corretta identificazione del paziente
}

\author{
Roberta Gallo, ${ }^{1,2}$ Fabio Bertoncini, ${ }^{2,3}$ Claudia Gatta ${ }^{2,4}$ \\ ${ }^{1}$ Associazione ANÍMO; ${ }^{2}$ ASL Biella; ${ }^{3}$ Gruppo Ricerca Associazione ANÍMO; ${ }^{4}$ Past-President Associazione ANÍMO, Italia
}

Nel settore sanitario, la corretta identificazione dei pazienti è un obiettivo da perseguire con priorità assoluta al fine di prevenire eventuali errori nella somministrazione di farmaci, errori durante esami strumentali o errori nelle trasfusioni. Promuovere la cultura alla sicurezza delle cure è un obiettivo raggiungibile solo attraverso la garanzia di sorveglianza di tutte le fasi di ogni processo, a partire proprio dall'identificazione dei pazienti.

L'identificazione del paziente deve essere fatta durante tutto il processo assistenziale, in diversi momenti così da creare maggiore sicurezza nel percorso di cura facilitando il riconoscimento del paziente.

La sicurezza dei pazienti è considerata una priorità per i sistemi sanitari in tutto il mondo. I dati disponibili dimostrano che, in media, gli incidenti correlati all'assistenza sanitaria influenzano 8 su 100 pazienti, e 4 incidenti su 5 sono prevenibili. ${ }^{1}$

Gli approcci per identificare correttamente i pazienti, utilizzati nei sistemi sanitari di tutto il mondo sono molto differenti: strumenti, risorse e linee guida strutturati per miglioramento la sicurezza del paziente attraverso un'identificazione univoca e tracciabile. ${ }^{1}$

Attualmente la tecnologia sembra essere la risposta principale per risolvere il problema della corretta identificazione del paziente; ${ }^{2}$ proprio all'interno delle opportunità tecnologiche si rintracciano tre differenti modelli strutturati per il riconoscimento dei pazienti:

- La tecnologia dei codici a barre viene utilizzata con lo scopo principale di legare la documentazione ad un paziente particolare, per esempio, durante la prescrizione e la somministrazione di farmaci, la richiesta e l'esecuzione di esami e la somministrazione di sangue o emoderivati.

Corrispondente: Fabio Bertoncini, ASL BI, Medicina d'urgenza, via dei Ponderanesi 2, Ponderano (BI), Italia.

E-mail: fabio.bertoncini@hotmail.it

Articolo pubblicato secondo la Creative Commons Attribution NonCommercial 4.0 License (CC BY-NC 4.0).

${ }^{\circ}$ Copyright: the Author(s), 2019

Licensee PAGEPress, Italy

QUADERNI - Italian Journal of Medicine 2019; 7(6):61-62
- I tag vengono utilizzati quando la necessità principale è quella di monitorare il paziente stesso, per esempio in ambienti geriatrici;

- Le tecnologie biometriche (ad esempio la scansione dell'iride) vengono implementate al fine di identificare l'individuo che si presenta in un servizio, per esempio nei processi di distribuzione del metadone. $^{2}$

Uno studio condotto all'interno di un azienda sanitaria inglese in cui l'identificazione dei pazienti veniva effettuata attraverso bracciali identificativi, ${ }^{3}$ analizzando un intero biennio, ha registrato 236 incidenti e near misses correlati alla mancanza di braccialetti con codici a barre o a braccialetti contenenti informazioni errate. ${ }^{3}$

La letteratura evidenzia la necessità di identificare strategie efficaci per migliorare i processi di assistenza: per l'identificazione del paziente, laddove non sono disponibili sistemi di controllo elettronici, è necessario utilizzare almeno due modalità differenti per verificarne l'identità, attraverso il nome, il cognome e data di nascita. Questo permette di assicurarsi che ogni paziente ottenga i trattamenti corretti. ${ }^{4}$

Per l'uso corretto di farmaci: conoscere le informazioni corrette circa i farmaci del paziente. Confrontare i farmaci assunti dal paziente con i nuovi farmaci somministrati. Assicurarsi che il paziente conosca i farmaci da assumere quando sarà al domicilio. ${ }^{4}$

All'interno dell'ambito chirurgico, laddove gli ambienti operatori già prevedono check-list serrate e procedure codificarte, gli errori quali interventi chirurgici nel paziente sbagliato o in parte del corpo sbagliata rappresentano invece eventi particolarmente gravi, che possono essere determinati da diversi fattori, tra cui spicca la non corretta identificazione del paziente o del sito chirurgico: nonostante non si disponga di dati ufficiali di incidenza, si stima che le procedure chirurgiche su un sito o su paziente erroneamente identificato non siano al livello di uno zero desiderato. ${ }^{5}$

Fortunatamente, gli interventi e le strategie disponibili, possono ridurre significativamente il rischio di errori nell'identificazione del paziente.

Indipendentemente dalla tecnologia o dall'approccio utilizzato per identificare i pazienti, pianificare attentamente i processi di cura in ogni loro fase strutturando passaggi per un'adeguata identificazione 
del paziente prima di ogni intervento medico, permette di fornire cure più sicure con meno errori. ${ }^{6,7}$

I codici a barre, come accennato in precedenza, sono la forma più familiare di identificazione, attaccati ad un braccialetto, posseggono le informazioni del paziente e tutti i dati possono essere accessibili attraverso un dispositivo in grado di leggerli.

Anche un identificatore del paziente biometrico ( $\mathrm{ad}$ esempio una scansione dell'iride), potrebbe essere codificato come barcode e indossato come un braccialetto.

I codici a barre vengono in genere utilizzati con un'applicazione in cui le informazioni codificate vengono strutturate come record in un database che permette di ricercare informazioni più dettagliate sul paziente che si sta visualizzando. ${ }^{2}$

Gli aspetti positivi che la letteratura identifica nell'utilizzo di codici a barre per l'identificazione dei pazienti sono: i) facilità di utilizzo; ii) notorietà e diffusione della tecnologia; iii) economicità del metodo di codifica delle informazioni di testo; iv) facilità di lettura da parte di dispositivi elettronici poco costosi; v) rapidità della possibilità di raccolta dati; vi) facilità di stampa.

I limiti: i) il lettore di codici a barre richiede una superficie liscia per la lettura; ii) il codice a barre è illeggibile, senza dispositivo, al personale, pazienti e parenti; in caso di errata identificazione questa non potrà essere prontamente riconosciuta; ${ }^{4}$ iii) pur avendo un costo unitario basso, il numero di lettori necessari per dare avvio all'utilizzo ad un intero reparto può essere significativo; iv) possibilità di difficoltà fisiche nell'ottenere una superficie lineare di lettura per il lettore di codici a barre in alcuni reparti. Gli esempi includono terapie intensive, dove i pazienti potrebbero avere numerosi bracciali, infusioni e monitor collegati e reparti neonatali, dove la dimensione e la curvatura del braccialetto potrebbe essere troppo piccolo per consentire al dispositivo di leggere il codice a barre. ${ }^{2}$

Tutta la letteratura, le procedure interne alle organizzazioni, le norme di buon senso, così come le buone pratiche, sono concordi nell'affermare che i paradigmi più importanti per abbattere la possibilità di commettere errori di identificazione dei pazienti all'interno dei processi di assistenza infermieristica comprendono: la diffusione della cultura della doppia identificazione effettuata da due operatori indipendenti, la costante sensibilizzazione del personale sanitario, l'implementazione di tecnologie che garantiscano e favoriscano la sicurezza del paziente (identificatori tecnologici) e la condivisione di soluzioni personalizzate che si fondano sull'organizzazione e sulle risorse disponibili e tendano costantemente al miglioramento della sicurezza dei pazienti stessi. ${ }^{8}$

\section{Bibliografia}

1. World Health Organization. Regional Office for the Eastern Mediterranean. Patient safety tool kit. 2015. Available from: http://apps.who.int/iris/bitstream/handle/10665/ 195709/EMROPUB_2015_EN_1856.pdf?sequence=1\&i sAllowed $=y$

2. Australian Commission on Safety and Quality in Health Care. Technology Solutions to Patient Misidentification. Report of Review. 2008. Available from: https://www. safetyandquality.gov.au/wp-content/uploads/2012/01/ 19794-TechnologyReview1.pdf

3. NHS Improvement. Learning from patient safety incidents. 2017. Available from: http://www.npsa.nhs.uk/ site/media/documents/1440_Safer_Patient_Identification_SPN.pdf

4. The Joint Commission. National Patient Safety Goals. 2019. Available from: https://www.jointcommission.org/ assets/1/6/2019_NCC_NPSGs_final.pdf

5. Ministero della salute. Raccomandazione per la corretta identificazione dei pazienti, del sito chirurgico e della procedura. Raccomandazione n. 3. 2008. Available from: http://www.salute.gov.it/imgs/C_17_pubblicazioni_587_ allegato.pdf

6. Greenly M. Helping Hippocrates: a cross-functional approach to patient identification. Joint Commission. J Qual Patient Safety 2006;32:463-9.

7. McDonald CJ. Computerization can create safety hazards: a bar-coding near miss. Ann Intern Med 2006; 144:510-6.

8. Lippi G, Mattiuzzi C, Bovo C, Favaloro EJ. Managing the patient identification crisis in healthcare and laboratory medicine. Clin Biochem 2017;50:562-7. 


\title{
Fase diagnostica: evitare misdiagnosi e ritardi
}

\author{
Matteo Giorgi-Pierfranceschi \\ Medicina Interna, Istituti Ospitalieri di Cremona, ASST-Cremona, Cremona, Italia
}

\section{Introduzione}

In ambito clinico i due maggiori prodotti del processo decisionale, clinical decision making, sono la diagnosi e il programma terapeutico. Se la diagnosi è corretta, anche la terapia ha ottime probabilità di essere efficace. In realtà alcuni dati della letteratura stimano che gli errori diagnostici siano nell'ordine del 10-15\%, e questo dato è sicuramente superiore a quanto percepito dai clinici nella loro pratica quotidiana. Secondo alcune stime le specialità maggiormente soggette a errori nella fase diagnostica sono la medicina d'urgenza, la medicina generale (di famiglia) e la medicina interna, mentre in ambito radiologico e anatomo-patologico questa percentuale scende intorno al $2 \%{ }^{1}$

\section{Fattori che influenzano gli errori in fase diagnostica}

L'errore diagnostico solo raramente è causato da una scarsa conoscenza della patologia o del protocollo clinico, nella maggior parte dei casi esso è dovuto a un processo cognitivo che non prende in considerazione quella determinata possibilità clinica, nonostante molti indizi orientino alla diagnosi corretta (errore cognitivo). I bias che portano a una diagnosi errata, ritardata o omessa, spesso dipendono dall'attitudine specialistica del medico, per esempio difronte a un sintomo aspecifico come l'emottisi, un infettivologo potrebbe essere più orientato a escludere una tubercolosi (TBC), mentre un internista potrebbe pensare a una trombo-embolia polmonare (TEP). Altri errori in

Corrispondente: Matteo Giorgi-Pierfranceschi, U.O. Medicina Interna, Istituti Ospitalieri di Cremona, ASST-Cremona, viale Concordia 1, 26100 Cremona, Italia.

E-mail: magiopi65@gmail.com

Articolo pubblicato secondo la Creative Commons Attribution NonCommercial 4.0 License (CC BY-NC 4.0).

${ }^{\circ}$ Copyright: the Author(s), 2019

Licensee PAGEPress, Italy

QUADERNI - Italian Journal of Medicine 2019; 7(6):63-66 fase diagnostica possono essere influenzati da un quesito specifico con cui il paziente giunge all'attenzione del clinico, distogliendo l'attenzione da altri dati anamnestici rilevanti, ad esempio: emottisi e dispnea in paziente imunocompromesso: escludere TBC. A questo punto l'iter diagnostico potrebbe essere finalizzato all'esclusione dell'infezione e non a considerare tutte le cause di emottisi, che in quel paziente affetto anche da una malattia infiammatoria cronica, potrebbe essere dovuto a una TEP, poiché ne costituisce un importante fattore di rischio.

Uno studio del 2001, basato su reperti autoptici, dimostrava una misdiagnosi di TEP fatale nel $55 \%$ di una casistica di pazienti analizzati. ${ }^{2}$

Alcuni autori hanno preso in considerazione una serie di queste interferenze nella fase diagnostica del processo decisionale per eliminarle o almeno ridurle, utilizzando il termine di debiasing strategy. ${ }^{3}$ Nella pratica non è così semplice, soprattutto perché non sempre il decision maker è consapevole dei propri limiti di giudizio (pregiudizi), dovuti alla propria formazione clinica ed alla propria esperienza (case-mix), ma spesso anche a fattori dipendenti dall'organizzazione che possono comprendere la turnistica, la facilità di accesso alla diagnostica più avanzata, il sovraffollamento. Per evitare che il pregiudizio possa influenzare il decision-maker è necessario adottare un processo che permetta il passaggio da una modalità di ragionamento intuitiva, più immediata, basata sulle conoscenze, sull'esperienza e sulla consuetudine, ad una modalità analitica,${ }^{4}$ più riflessiva, basata su evidenze scientifiche (linee guida) che si possano integrare con le caratteristiche dell'organizzazione locale. In quest'ultimo contesto, sebbene non esistano evidenze che supportino l'utilizzo di strategie ben definite per la riduzione dei bias, i percorsi diagnostico terapeutici assistenziali (PDTA) potrebbero essere definiti una debiasing strategy, soprattutto se essi vengano strutturati partendo dai sintomi clinici di presentazione, più che dalle patologie, perché questo prescinde da una diagnosi eziologica che potrebbe essere formulata in modo errato dal primo operatore che avvia il percorso, per esempio: la definizione di un PDTA per il paziente con dispnea acuta o con insufficienza respiratoria, potrebbe essere più efficace nel ridurre i bias 
piuttosto che un PDTA per $B P C O$ riacutizzata. $\mathrm{Al}$ momento comunque non esistono evidenze che dimostrino questa tesi.

\section{Il ruolo delle tecniche di imaging}

Parlando di fase diagnostica è necessario considerare tutti gli strumenti che possono essere utili alla definizione di una patologia. Prendendo ad esempio l'embolia polmonare, vediamo come nella fase diagnostica siano utilizzati differenti strumenti diagnostici che comprendono scores di rischio, test di laboratorio e imaging, che devono essere utilizzati secondo una sequenza necessaria a razionalizzare il loro impiego, che è mostrata nelle linee guida dell'european society of cardiology (ESC) per la gestione della tromboembolia polmonare. ${ }^{5}$

\section{Valutazione clinica pre-test}

Wells score e Geneva score, ${ }^{5}$ sono strumenti che permettono di stratificare i pazienti con sospetto di embolia polmonare in base alle caratteristiche di presentazione (sintomi, parametri clinici, fattori di rischio), e li collocano in categorie di rischio di avere l'embolia polmonare, prima che siano eseguiti i test di laboratorio e gli esami strumentali. Questo passaggio si rende necessario perché secondo la legge di Bayes la probabilità di diagnosticare una malattia dopo un test non dipende soltanto dalla sensibilità $\mathrm{e}$ dalla specificità del test, ma anche dalla probabilità clinica del paziente di avere quella malattia prima dell'esecuzione del test, di conseguenza questa stratificazione conferisce maggiore appropriatezza diagnostica, tanto che se la probabilità pre-test è bassa ed il dosaggio dei $\mathrm{D}$-dimeri è negativa, il paziente non deve proseguire l'iter diagnostico e non sono necessari ulteriori test.

\section{Test diagnostici}

Per la diagnosi di TEP abbiamo a disposizione numerosi esami diagnostici di imaging, il gold-standard per la diagnosi di TEP è l'angiografia polmonare, ma nella pratica clinica viene surrogata dalla TAC torace con mezzo di contrasto, mentre la scintigrafia polmonare ventilo-perfusionale, l'ecocardiogramma, l'ecografia compressiva (CUS) venosa degli arti inferiori sono indagini utilizzate in situazioni in cui l'angio-TAC polmonare potrebbe non essere sempre disponibile o controindicata (per esempio allergia m.d.c.), e forniscono dati importanti per la conferma o per l'esclusione della diagnosi. Ma per utilizzare correttamente $i$ test diagnostici è necessario sapere qual è la loro accuratezza diagnostica nei confronti non solo di una patologia, ma di una popolazione a rischio alto o basso di avere quella patologia, inoltre nella diagnosi di TEP, alcuni esami hanno un valore predittivo positivo molto elevato, come l'angio-TC polmonare, che se positiva, conferma la diagnosi nella totalità dei casi. Invece quando l'angio-TC risulta negativa, non può escludere la TEP nel $100 \%$ dei pazienti con alta probabilità pre-test, ${ }^{5}$ perché ha una buona accuratezza fino ai rami arteriosi polmonari sub-segmentari. Altri esami invece espletano il loro potere diagnostico attraverso un valore predittivo negativo molto alto, come la scintigrafia polmonare perfusionale, che se negativa, è in grado di esclude una TEP indipendentemente dalla probabilità clinica pre-test.

La diagnosi di embolia polmonare utilizzata come esempio in questo paragrafo, mostra come la stratificazione dei pazienti in base alla probabilità clinica $\mathrm{e}$ la conoscenza dell'accuratezza dei diversi test diagnostici per questa patologia, siano alla base di una corretta impostazione dell'iter diagnostico del paziente.

\section{Ecografia point of care}

Recenti evidenze assegnano un ruolo importante all'ecografia point of care nella diagnosi di TEP, infatti l'ecografia del torace (LUS), l'ecocardiogramma e la CUS degli arti inferiori utilizzate in modo combinato nel paziente con sospetto di TEP permettono di raggiungere una buona sensibilità e specificità, che risulta superiore alle diverse tecniche usate singolarmente (Tabella 1$)^{6}$

L'impiego dell' ecografia point of care è una tecnica che può essere diffusa capillarmente nei dipartimenti di emergenza e nei reparti di medicina per, e consente un rapido inquadramento clinico del paziente critico attraverso la visualizzazione di finestre ecografiche standardizzate. Questa tecnica permette di escludere o avvalorare ipotesi cliniche direttamente al letto del paziente, con adeguata riproducibilità e accuratezza. ${ }^{7}$

Tabella 1. Sensibilità e specificità delle tecniche ecografiche nel paziente con sospetto di TEP. ${ }^{6}$

\begin{tabular}{lcccc}
\hline & Echocardiography & CUS & LUS & Multiorgan \\
\hline Sensitivity \% & 32.7 & 52.7 & 60 & 90 \\
\hline Specificity \% & 90.9 & 97.6 & 95.9 & 86.2 \\
\hline
\end{tabular}

CUS, compressive ultrasound; LUS, lung ultrasound; multiorgan, uso combinato delle tre tecniche. 


\section{Conclusioni}

La fase diagnostica costituisce un momento cruciale nell'iter di cura del paziente internistico acuto, la tecnologia ci offre strumenti sempre più efficienti che devono esser impiegati con appropriatezza per evitare esami non necessari ed eseguire tempestivamente quelli più indicati. La conoscenza delle linee guida e la loro implementazione nell'ambito delle differenti realtà organizzative (PDTA), devono essere finalizzate alla riduzione del rischio clinico e al miglioramento degli standard di cura. L'ecografia point of care può costituire uno strumento prezioso per un corretto e rapido inquadramento clinico del paziente critico.

\section{Bibliografia}

1. Berner ES, Graber ML. Overconfidence as a cause of diagnostic error in medicine. Am J Med 2008;121: S2-S23.

2. Pineda LA, Hathwar VS, Grand BJ. Clinicalsuspicion of fatal pulmonary embolism. Chest 2001;120:791-5.

3. Croskerry P. A universal model for diagnostic reasoning. Acad Med 2009;84:1022-8.

4. Croskerry P, Singhal G, Mamede S. Cognitive debiasing 1: origins of bias and theory of debiasing. BMJ Qual Saf 2013;22:ii58-ii64.

5. Konstantinides SV, Torbicki A, Agnelli G, et al. 2014 ESC guidelines on the diagnosis and management of acute pulmonary embolism. Task Force for the Diagnosis and Management of Acute Pulmonary Embolism of the European Society of Cardiology (ESC). Eur Heart J 2014;35:3033-69.

6. Nazerian P, Vanni S, Volpicelli G, et al. Accuracy of point-of-care multiorgan ultrasonography for the diagnosis of pulmonary embolism. Chest 2014;145:950-7.

7. Wittenberg M. Will ultrasound scanners replace the stethoscope? BMJ 2014;348:g3463. 


\title{
Sicurezza in terapia farmacologica
}

\author{
Elisa Romano \\ Clinical Risk Manager, ASL 5 Liguria, La Spezia, Italia
}

\section{Premessa}

Il miglioramento della sicurezza dei farmaci ad alto rischio è uno degli International Patient Safety Goals indicati dalla Joint Commission International nel 1992. Farmaci ad alto rischio sono quelli che hanno una potenziale tossicità, un basso indice terapeutico e un'alta possibilità di interazioni (ad esempio anticoagulanti, antiaritmici, oppioidi, benzodiazepine ev, insulina, ipoglicemizzanti orali). ${ }^{1}$ Legate alla natura del farmaco sono le reazioni avverse, che vanno segnalate alla Farmacovigilanza e gli effetti collaterali, che devono essere valutati nel contesto clinico. La maggior parte dei danni è legata a pratiche non sicure e a errori, tendenzialmente prevenibili, che avvengono nelle diverse fasi del processo di gestione della terapia farmacologica.

Medication without harm è il terzo Patient Safety Challenge del WHO, dopo l'Igiene delle mani e la Sicurezza in chirurgia (Clean Care is Safer Care e Safe Surgery Saves Lives). Lanciato nel 2017, si propone l'obiettivo di ridurre del $50 \%$ in 5 anni i danni gravi da errori prevenibili in terapia farmacologica. ${ }^{2}$

\section{Le raccomandazioni ministeriali di sicurezza nell'uso dei farmaci}

Il Ministero della Salute pubblica e diffonde le Raccomandazioni di sicurezza, ${ }^{3}$ relative a situazioni pericolose che possono determinare gravi o fatali eventi avversi. La maggior parte di esse sono nate in seguito a drammatici fatti di cronaca nera sanitaria, come ad esempio la Raccomandazione n. 1, relativa alla corretta gestione della terapia con soluzioni con-

Corrispondente: Elisa Romano, Clinical Risk Manager, Via Manin 17, 19121 La Spezia, Italia.

E-mail: elisaromano@virgilio.it

Articolo pubblicato secondo la Creative Commons Attribution NonCommercial 4.0 License (CC BY-NC 4.0).

${ }^{\circ}$ Copyright: the Author(s), 2019

Licensee PAGEPress, Italy

QUADERNI - Italian Journal of Medicine 2019; 7(6):67-74 centrate di potassio, pubblicata e diffusa dopo la morte di un bimbo, avvenuta in seguito allo scambio di una fiala di soluzione fisiologica con una di $\mathrm{KCl}$. L'evento fatale avvenne nel 2005, molto prima della pubblicazione della Raccomandazione, ma l'infermiera responsabile venne condannata con sentenza definitiva nel 2015, poiché già nel marzo 2004 era stato pubblicato un documento che conteneva nei suoi allegati le linee guida relative alla corretta gestione dei farmaci ad alto rischio. ${ }^{4}$

Le Raccomandazioni di sicurezza pubblicate dal Ministero vengono trasferite, a livello aziendale, in procedure, protocolli o istruzioni operative. Allo stato attuale sono state pubblicate, nell'arco di dieci anni, 18 raccomandazioni. Di queste ben 6 sono relative alla sicurezza in terapia farmacologica, a testimonianza della notevole rilevanza della possibilità di errori (Tabella 1$)$.

Le prime tre (1, 7 e 14) sono inserite nel Manuale Qualità e Sicurezza nell'uso dei Farmaci pubblicato in settembre 2010.

La Raccomandazione $\mathrm{n}^{\circ} 7$ definisce le singole responsabilità nell'ambito del processo terapeutico, affidando esclusivamente al medico la fase della Prescrizione e imponendo che tutte le fasi successive siano tracciate sulla Scheda Terapeutica Unica, parte integrante della cartella clinica. Nella raccomandazione sono evidenziati i requisiti fondamentali dello strumento, che permette di tracciare tutti i passaggi del processo con le firme dei medici prescrittori e degli infermieri somministratori, i quali, in caso di mancata assunzione del farmaco sono tenuti anche ad evidenziarne il motivo.

Anche se solo il $21 \%$ di tutti gli errori in terapia è attribuibile alla fase della prescrizione, in essa vi è una maggiore probabilità di evento avverso con danno severo. ${ }^{5}$ Le classi di farmaci coinvolte sono quelle maggiormente utilizzate nelle corsie di Medicina Interna (Tabella 2). Particolare attenzione va assicurata alla Terapia Anticoagulante, sia con i vecchi che con i nuovi farmaci (Tabella 3).

\section{La Scheda Terapeutica Unica Universale}

La necessità di eliminare i numerosi errori legati ad una cattiva compilazione delle Schede Terapeutiche 
Uniche presenti nella nostra ASL, anche se esse erano aderenti ai requisiti previsti dalle Raccomandazioni Ministeriali, ci ha suggerito l'elaborazione di un modello unico per tutti i reparti di degenza, con requisiti di sicurezza talmente elevati da rendere veramente difficile sbagliare (Figure 1 e 2).

Le principali criticità (evidenziate da Audit, Safety walk around e Incident Reporting) riscontrate dalla SS Risk Management della nostra ASL, erano relative ad errori nella prescrizione di farmaci ad alto livello di attenzione e alla mancata convalida giornaliera della terapia. A queste si aggiungevano prescrizioni spesso incomplete, mancata o non corretta prescrizione della ossigenoterapia e mancanza della doppia firma per le infusioni di soluzioni di K, come previsto dalla raccomandazione $\mathrm{n}$. 1. Inoltre la presenza di STU diverse da reparto a reparto induceva ad errori in caso di ricoveri fuori reparto o sostituzioni con personale non abituato a quel tipo di modello.

La STU universale (Figure 1 e 2), stampata in A3, è articolata su 6 giorni e prevede che il medico firmi singolarmente la prescrizione e la sospensione dei far- maci. La conferma della terapia giornaliera si effettua con una firma unica, valida fino alle ore 8 del giorno successivo. Gli infermieri siglano la terapia in calce ad ogni sezione. In caso di infusione di soluzioni contenenti potassio è prevista la firma di due operatori (anche entrambi infermieri).

Nella scheda sono riportati sette possibili orari di somministrazione ma, per una migliore organizzazione del lavoro infermieristico, si consiglia di accorpare le sessioni di terapia: per le Medicine lo schema consigliato è $8,14,21$ (eccetto che per insuline e ASA ai pasti).

Sono presenti tre sezioni relative alle vie di somministrazione (orale, endovenosa e intramuscolare/sottocute/transdermica/sublinguale/altro), quattro sezioni relative ad alcuni farmaci ad alto livello di attenzione (antidiabetici, anticoagulanti, nutrizione artificiale, ossigeno), le sezioni Terapia al Bisogno e Terapia Estemporanea, una sezione per la Dieta.

L'intestazione del lato A contiene logo, barcode, dati anagrafici, allergie e legenda, nel lato B sono presenti solo i dati anagrafici.

Tabella 1. Raccomandazioni Ministeriali relative alla sicurezza farmacologica.

\begin{tabular}{cll}
\hline $\mathrm{n}^{\circ} 1$ & Corretto utilizzo delle soluzioni concentrate di KCL & $3 / 2008$ \\
\hline $\mathrm{n}^{\circ} 7$ & Prevenzione della morte, coma o grave danno derivati da errori in terapia farmacologica & $3 / 2008$ \\
\hline $\mathrm{n}^{\circ} 12$ & Prevenzione errori in terapia con Farmaci LASA - LOOK ALIKE / SOUND ALIKE & $8 / 2010$ \\
\hline $\mathrm{n}^{\circ} 14$ & Prevenzione errori in terapia con Farmaci Antineoplastici & $11 / 2012$ \\
\hline $\mathrm{n}^{\circ} 17$ & Raccomandazione per la Riconciliazione della Terapia Farmacologica & $12 / 2014$ \\
\hline $\mathrm{n}^{\circ} 18$ & Prevenzione errori in terapia conseguenti a uso abbreviazioni, acronimi, sigle e simboli & $9 / 2018$ \\
\hline
\end{tabular}

Tabella 2. Classi di farmaci coinvolte negli eventi avversi attribuibili ad errori durante la fase di prescrizione (Raccomandazione n. 7, Marzo 2008).

\begin{tabular}{ll}
\hline Ipoglicemizzanti & $28,7 \%$ \\
\hline Cardiovascolari & $18,6 \%$ \\
\hline Anticoagulanti & $18,6 \%$ \\
\hline Diuretici & $10,1 \%$ \\
\hline
\end{tabular}

Tabella 3. Eventi avversi da terapia anticoagulante.

Dati estratti dal Database Nazionale Danese dei Pazienti Anticoagulati, anno $2014^{6}$

Segnalati 147 eventi avversi (7 fatali, 83 severi, 52 significativi, 5 non significativi)

1. Il $78 \%$ degli errori è avvenuto nella fase di prescrizione

2. Tutti gli eventi fatali e l' $^{\prime} 84 \%$ di quelli severi erano stati determinati da un errore di prescrizione

3. Tutti gli eventi fatali e $1^{\prime} 82 \%$ di quelli severi sono avvenuti nella fase di transizione

4. 5 su $7(71 \%)$ di quelli fatali e il $40 \%$ di quelli severi erano riconducibili a un eccesso di dose

5. $79 \%$ degli errori è avvenuto nelle transizioni di cura (ingresso, dimissione e passaggio in chirurgia)

6. La dimissione è risultata la più critica delle tre, per tutte l'errore più comune è stato l'eccesso di dose 
Particolare attenzione deve rivestire la sezione della Terapia al Bisogno, a maggior rischio di errori per errata comunicazione o interpretazione delle consegne. Dopo aver accertato l'assenza di allergie/intolleranze e aver verificato le necessarie cautele e le eventuali controindicazioni vanno riportati, in aggiunta alle indicazioni ordinarie di una corretta prescrizione, $i$ sintomi clinici e i parametri di riferimento che condizionano la somministrazione del farmaco, ossia l'intensità dei sintomi e/o reiterazione o durata degli stessi e la correlazione della somministrazione con indici misurati attraverso scale valutative (dolore), segni apprezzabili oggettivamente (temperatura corporea, pressione arteriosa), parametri biologici (controllo glicemico). Obbligatorio indicare il limite di posologia giornaliera e l'intervallo di tempo minimo tra una somministrazione e l'altra. (Esempio di prescrizione corretta: Paracetamolo $500 \mathrm{mg}$ compresse, $1 \mathrm{cp} \mathrm{se} \mathrm{TC}>37.8^{\circ} \mathrm{C}$, $\max 1 \mathrm{cp}$ ogni 8 ore). La Terapia al bisogno va confermata ogni giorno, per mantenere un'assidua vigilanza sulle somministrazioni. In caso di mancata/incompleta indicazione dei parametri di riferimento e dei limiti di esecuzione, difficoltà interpretative o rilievo di modifiche delle condizioni del paziente, l'infermiere deve chiedere una rivalutazione medica. La prescrizione di farmaci all'occorrenza può essere effettuata solo nei casi in cui esistano parametri oggettivi rilevabili, ma non agitazione o insonnia. Non vanno prescritti al bisogno farmaci che possono causare depressione respiratoria, ad eccezione degli oppioidi per pazienti con dolore neoplastico (es. dose rescue nello schema di terapia antalgica fissa o nella fase di titolazione).

\section{Ricognizione e riconciliazione della terapia}

Errori terapeutici possono avvenire in qualsiasi momento venga aggiornata o modificata la terapia. Tali errori, detti anche discrepanze non intenzionali, possono interessare fino al $67 \%$ dei pazienti al momento del ricovero in Ospedale e fino al 50\% al momento della dimissione. ${ }^{6}$ Per prevenire questi errori è fondamentale effettuare un'adeguata ricognizione della terapia in corso e riconciliarla alla luce delle eventuali modifiche da applicare.
Già nel 2005 la Joint Commission International inserì la Medical Reconciliation tra gli obiettivi del $\mathrm{Na}$ tional Patient Safety Goal e la WHO considera la Riconciliazione Farmacologica una delle migliori strategie per garantire la sicurezza delle cure.

La Raccomandazione n. 17, pubblicata a dicembre 2014, affronta la criticità relativa a errori di terapia che possono avvenire nei momenti di transizione di cura, ad esempio al momento del ricovero o della dimissione o nell'ambito di un trasferimento tra reparti o ad altra struttura sanitaria. Le modalità operative della Ricognizione e della Riconciliazione sono sintetizzate nelle Tabelle 4 e 5.

\section{Live Survey FADOI-ANİMO}

Durante il XXIII Congresso Nazionale FADOI, tenutosi a Bologna in maggio 2018, è stata organizzata una giornata formativa dedicata alla Campagna OMS Medication without harm.

$\mathrm{Al}$ termine dei lavori è stata effettuata, mediante la piattaforma on-line Mentimeter, una Live Survey sull'applicazione delle Raccomandazioni Ministeriali per la Sicurezza Farmacologica e delle Buone Pratiche ad esse connesse, nell'ambito della propria realtà lavorativa. Il campione, anche se piccolo, rispecchiava le diverse figure coinvolte nel processo di gestione della Terapia Farmacologica ed era comunque rappresentativo di persone interessate e motivate al miglioramento della sicurezza.

Dalle risposte dei 114 intervistati (40 medici, 73 infermieri e 1 farmacista), sono emersi dati non rassicuranti: i) $8 \%$ degli intervistati lavora in reparti che non utilizzano la scheda terapeutica unica; ii) solo il $36 \%$ lavora in reparti con STU in formato elettronico; iii) il 44\% non utilizza le schede di ricognizione e riconciliazione; iv) solo il $19 \%$ proviene da reparti dotati di schede in formato elettronico; v) il $50 \%$ non ha in reparto un protocollo per la gestione delle infusioni di K; vi) solo il $23 \%$ utilizza il doppio controllo nella preparazione e somministrazione e la doppia firma sulla in STU; vii) il 50\% non ha in reparto elenco né contrassegni particolari per i Farmaci LASA (Lookalike/sound alike); viii) per quanto riguarda i farmaci

Tabella 4. Ricognizione: modalità operative.

\begin{tabular}{ll}
\hline Al momento del ricovero del paziente \\
\hline COSA: & $\begin{array}{l}\text { completa e accurata raccolta di informazioni sul paziente e sui medicinali che assume, compresi farmaci off-label (con relativa } \\
\text { motivazione ed indicazione), omeopatici, fisioterapici e integratori }\end{array}$ \\
CHI: & $\begin{array}{l}\text { il Medico } \\
\text { QUAN'immediatezza della presa in carico o al massimo entro } 24 \text { ore, tranne che in situazioni di emergenza o quando le informazioni } \\
\text { essenziali non siano immediatamente disponibili }\end{array}$ \\
COME: & $\begin{array}{l}\text { utilizzando la "SCHEDA RICOGNIZIONE TERAPIA FARMACOLOGICA - AMMISSIONE" } \\
\text { PERCHÈ: }\end{array}$
\end{tabular}


Tabella 5. Riconciliazione: modalità operative.

Al momento della transizione di cura

COSA: $\quad$ confronto della terapia del paziente con le prescrizioni ex novo/modifiche necessarie per l'attuale circostanza clinica

CHI: $\quad$ il Medico

QUANDO: appena disponibili i dati della Ricognizione, prima di eseguire la prescrizione farmacologica. Nei casi urgenti può essere espletata successivamente

COME: tramite la scheda "Ricognizione - Ammissione" e la "STU"

PERCHÈ: per individuare possibili incongruenze, sovrapposizioni, omissioni, interazioni, controindicazioni e confusione dovuta a farmaci Look-Alike/Sound-Alike (LASA), valutando note AIFA, farmaci del PTO, protocolli clinici e procedure aziendali

Al momento della prescrizione

COSA: decisioni terapeutiche relative all'evento che ha condotto al ricovero, impostate sulla base del processo clinico dinamico e della ricognizione/riconciliazione

CHI: $\quad$ il Medico

QUANDO: durante tutto il periodo di ricovero, con aggiornamento almeno ogni 24 ore, firmando convalide, variazioni e sospensioni

COME: utilizzando la "Scheda Terapeutica Unica", secondo la procedura

Al momento della dimissione

COSA: $\quad$ prescrizione terapeutica proposta in fase di dimissione, con eventuali modifiche rispetto alle terapie precedenti, che pone particolare attenzione alle modalità di assunzione e indica le motivazioni delle modifiche rispetto alla terapia in ingresso rilevata dalla Ricognizione

CHI: $\quad$ il Medico

QUANDO: alla preparazione della lettera di dimissione o trasferimento

COME: utilizzando la "SCHEDA RICONCILIAZIONE TERAPIA FARMACOLOGICA - DIMISSIONE" (Figure 3 e 4), da consegnata al paziente insieme alla lettera di dimissione o di trasferimento

PERCHÈ: facilita una comunicazione corretta tra tutti gli operatori e informa il paziente (familiari/caregiver) delle modifiche apportate al trattamento terapeutico, fornendone la motivazione.

ad altro rischio, il $68 \%$ riferisce la conservazione in appositi contenitori contrassegnati, ma solo il $16 \%$ utilizza la tecnica della doppia identificazione del paziente nella somministrazione e le tabelle di conversione nella prescrizione.

\section{Conclusioni}

La scarsa attenzione alle indicazioni di sicurezza previste dalle Raccomandazioni Ministeriali impone una maggiore diffusione delle tematiche relative al Risk Management da parte delle figure istituzionali ad esse preposte (Risk Manager, Direzione Sanitaria, Collegio di Direzione, Direzione Generale).

La Struttura Risk Management, supportata con un forte mandato dalla Direzione Strategica e dal Collegio di Direzione, deve sensibilizzare gli operatori sanitari alla cultura della sicurezza in maniera diretta, attraverso programmi di formazione capillare e successiva creazione, a livello periferico, di una rete di Referenti del Rischio Clinico. Nelle rispettive Unità Operative i Referenti organizzano e coordinano il Team della Sicurezza, di cui fanno parte il Direttore della Struttura e il Coordinatore Infermieristico o Tecnico. I Team della Sicurezza, oltre a vigilare sul ri- spetto della normativa e a garantire l'applicazione delle Buone Pratiche Cliniche nelle rispettive realtà, devono interagire tra di loro e collaborare in maniera attiva con la Struttura Centrale, segnalando i propri errori, identificando le criticità organizzative, proponendo soluzioni attuabili e ricercando ulteriori spazi di miglioramento della sicurezza delle cure.

\section{Bibliografia}

1. https://www.jointcommissioninternational.org/improve/ international-patient-safety-goals/?_ga=2.1676721. 1295428804.1552474824-928588191.1552474824

2. https://www.who.int/patientsafety/medication-safety/en/

3. http://www.salute.gov.it/portale/temi/p2_6.jsp?id= $250 \&$ area $=$ qualita $\&$ menu $=$ sicurezz

4. http://www.sanita24.ilsole24ore.com/print?uuid=ACnuwqkB

5. http://www.eepru.org.uk/prevalence-and-economic-burden-of-medication-errors-in-the-nhs-in-england-2/

6. Henriksen JN et al. Medication errors involving anticoagulants: Data from the Danish patient safety database. Pharmacol Res Perspect 2017;5:e00307.

7. https://www.bundesgesundheitsministerium.de/fileadmin/Dateien/3_Downloads/P/Patientensicherheit/WS62017/WS6_Kirke_Bonn_March_2017_Transitions_in care.pdf 


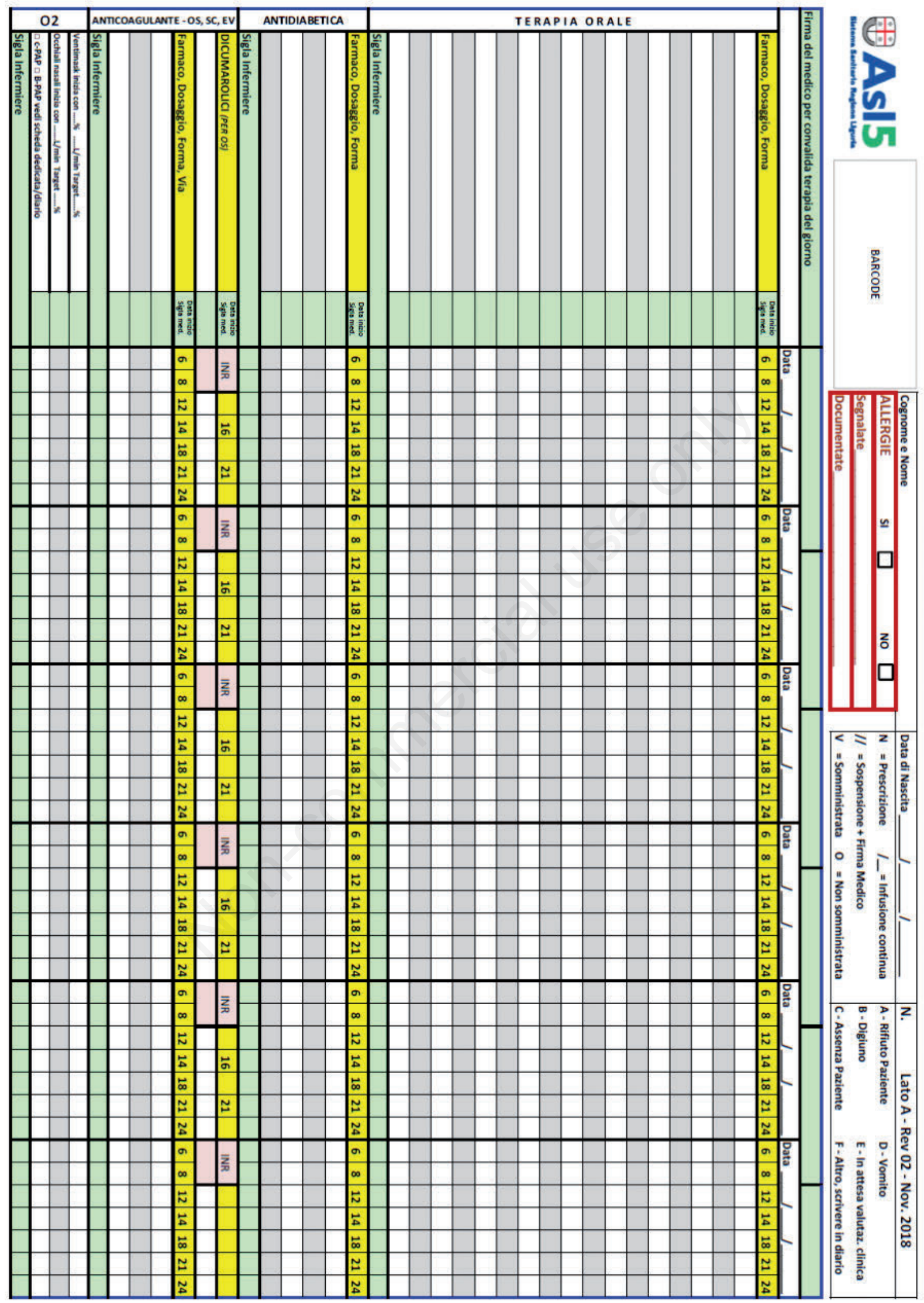

Figura 1. Modello di Scheda Terapeutica Unica Universale in vigore nell'ASL 5 - Regione Liguria. (Elaborazione Elisa Romano e Micaela La Regina). LATO A. 


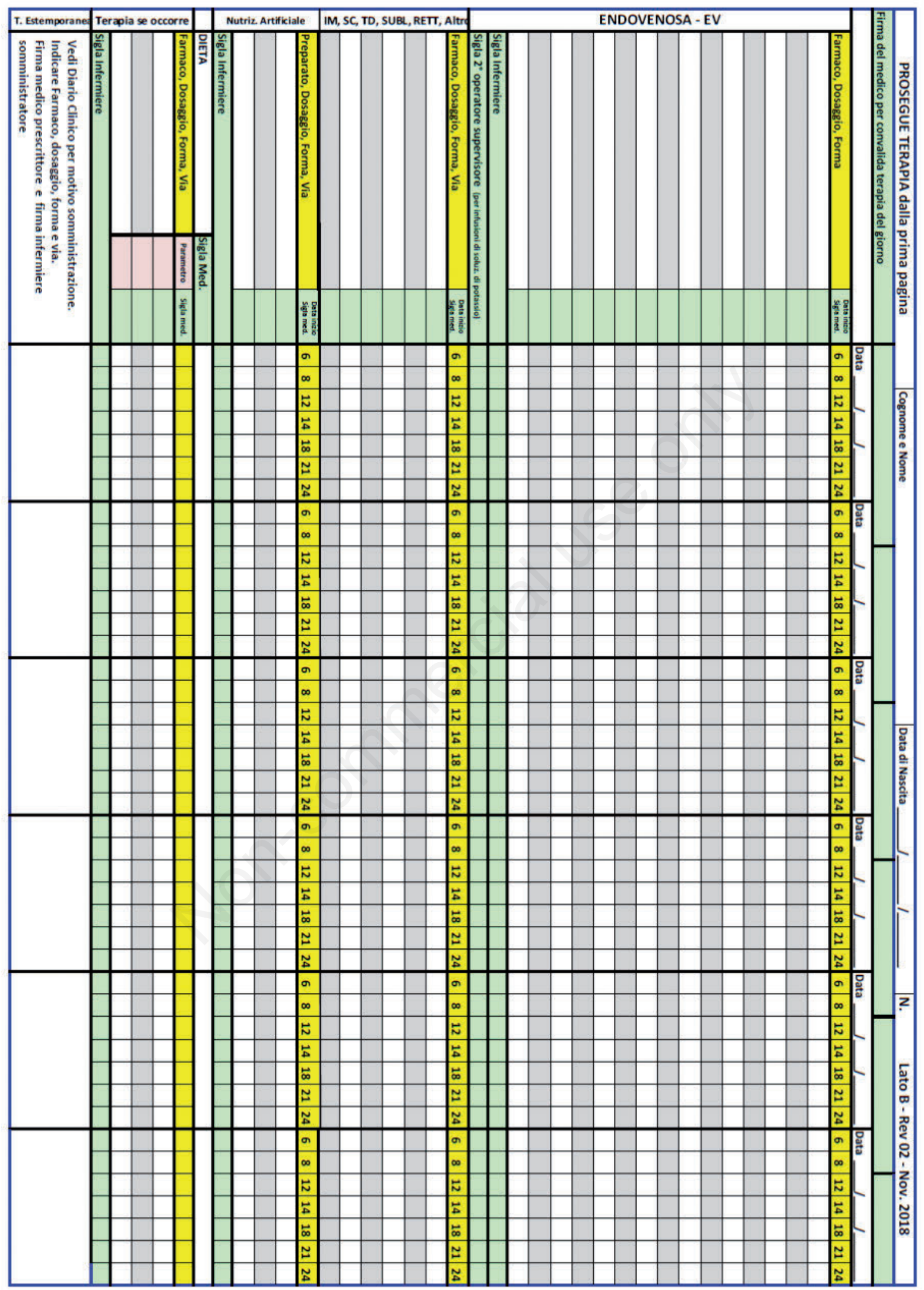

Figura 2. Modello di Scheda Terapeutica Unica Universale in vigore nell'ASL 5 - Regione Liguria (Elaborazione Elisa Romano e Micaela La Regina). LATO B. 


\section{RICOGNIZIONE TERAPIA FARMACOLOGICA}

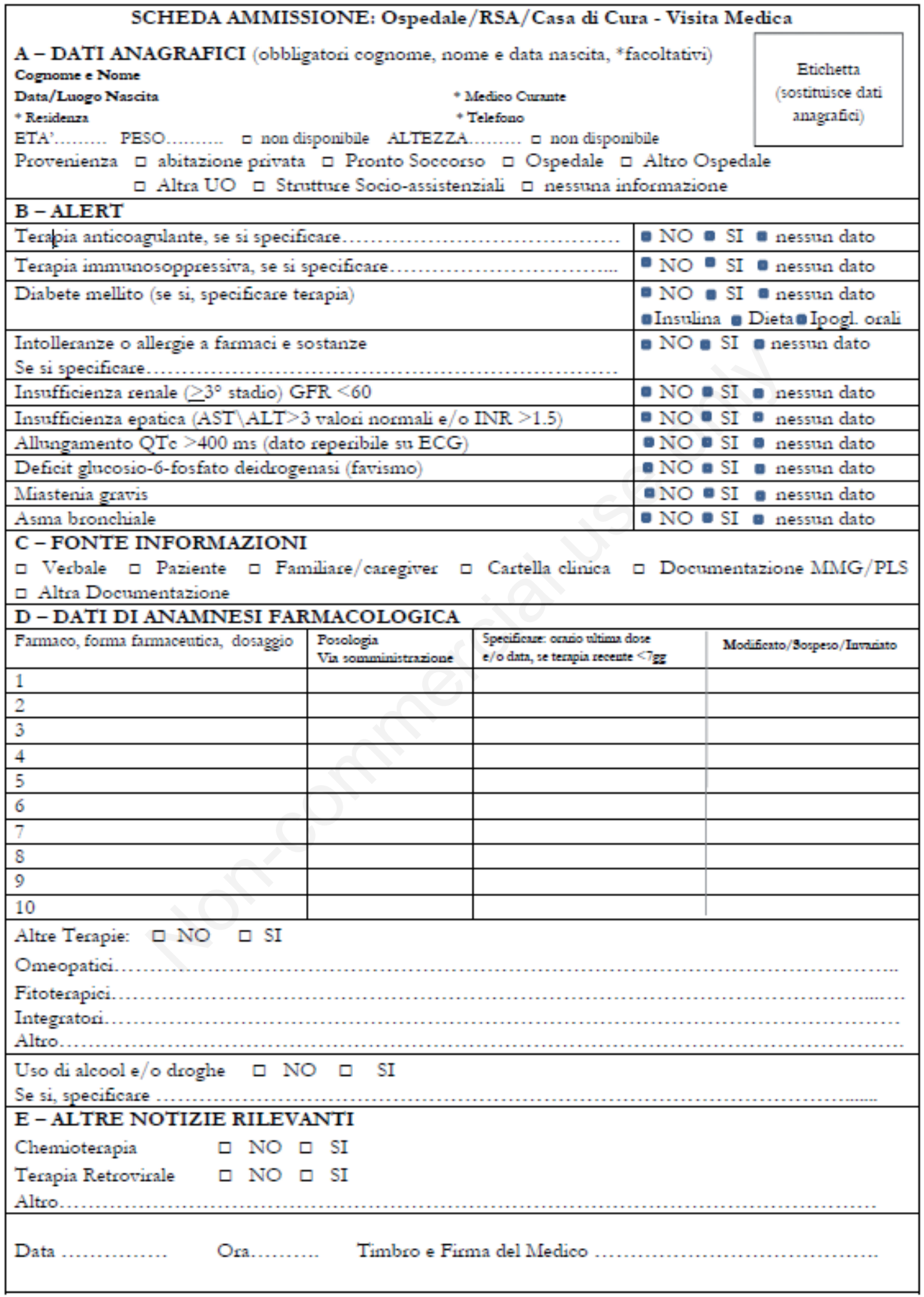

Figura 3. Modello di Scheda di Ricognizione della Terapia Farmacologica. LATO A. 


\section{RICONCILIAZIONE TERAPIA FARMACOLOGICA}

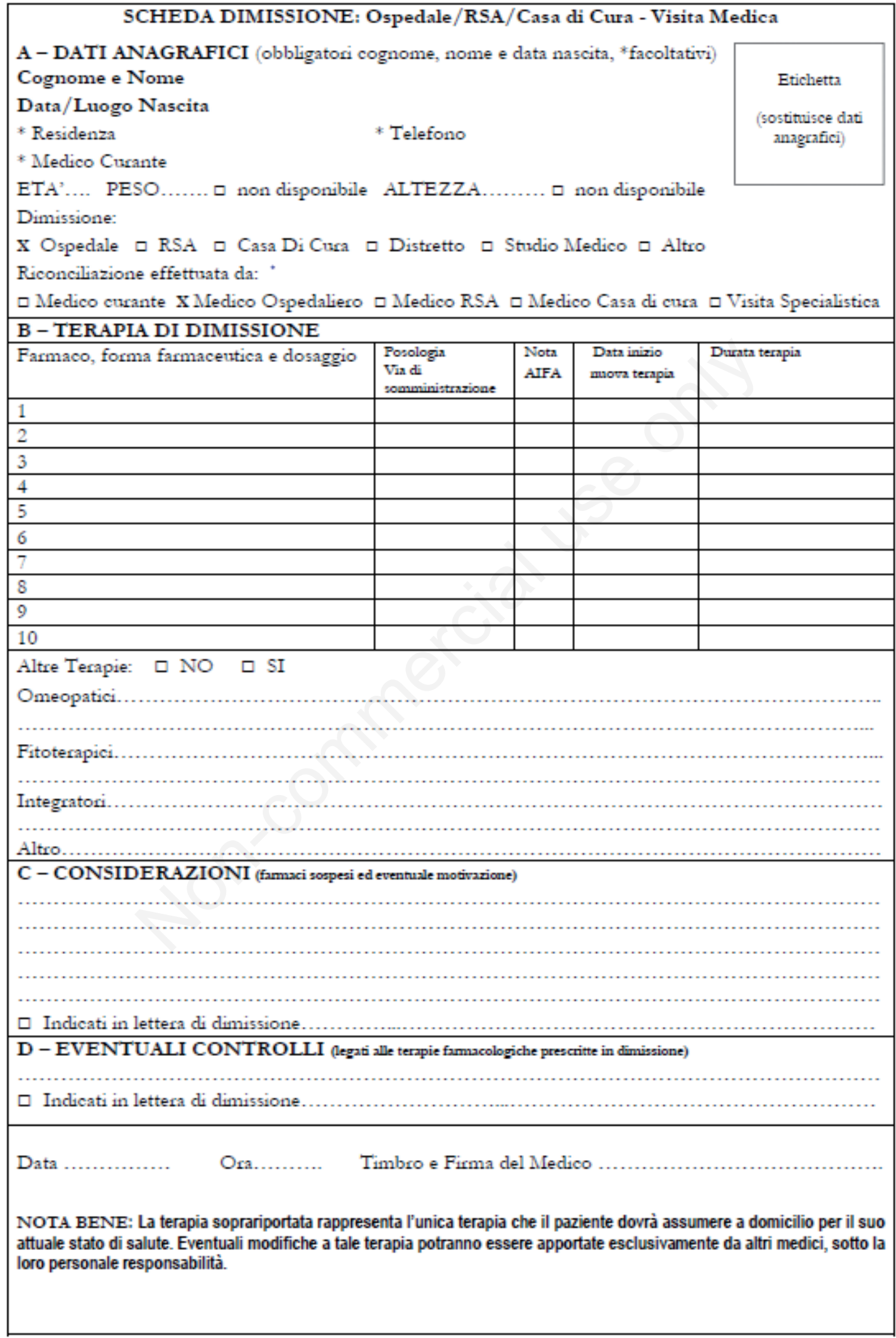

Figura 4. Modello di Scheda di Riconciliazione della Terapia Farmacologica. LATO B. 


\title{
Novità nella prevenzione delle cadute
}

\author{
Roberta Re, ${ }^{1}$ Giuseppina Ferrotti ${ }^{2}$ \\ ${ }^{1}$ Dirigente Medico in Medicina Interna, AOU Maggiore della Carità Novara; ${ }^{2}$ CPSE Medicina Interna II AOU Maggiore della \\ Carità Novara, Italia
}

\section{Introduzione}

Una notte qualunque nella Divisione di Medicina un paziente molto anziano, confuso, disorientato ed agitato cerca di scendere dal letto. Il letto è a circa 1,20 $\mathrm{mt}$ da terra, il paziente ha il catetere vescicale posizionato, l'ossigeno con erogatore nasale, la terapia infusionale endovena in continuo. La stanza è buia, il campanello è scivolato dal letto, ci sono solo 2 unità infermieristiche per 36 malati.

L'infermiere di turno ad un certo punto passa per il consueto giro degli ammalati e trova il paziente a terra, che geme. Il collega è dall'altro capo del reparto per cui l'infermiere solleva in fretta il paziente senza eseguire grosse valutazioni e va dall'altro infermiere per chiedere dove sono le cinghie di contenzione. Tempo un minuto è di nuovo in stanza e il paziente nuovamente per terra, prono. Una vasta ferita lacero contusa solca la fronte, il paziente non è più responsivo. Viene immediatamente posizionato nel letto e viene contattato il medico di guardia. Le condizioni diventano sempre più critiche, la tac cerebrale evidenzia una vasta emorragia cerebrale (il paziente era in terapia anticoagulante) e nonostante gli sforzi del Neurochirurgo purtroppo decede.

\section{Cosa non ha funzionato?}

Le cadute sono classificate come eventi sentinella. Il Ministero della Salute definisce un evento sentinella come: un evento avverso di particolare gravità, potenzialmente indicativo di un serio malfunzionamento

Corrispondente: Roberta Re, AOU Maggiore della Carità, 28100 Novara, Italia.

Tel.: +39.03213733412.

E-mail: roberta.re@tin.it

Articolo pubblicato secondo la Creative Commons Attribution NonCommercial 4.0 License (CC BY-NC 4.0).

${ }^{\circ}$ Copyright: the Author(s), 2019

Licensee PAGEPress, Italy

QUADERNI - Italian Journal of Medicine 2019; 7(6):75-84 del sistema, che può comportare morte o grave danno al paziente e che determina una perdita di fiducia dei cittadini nei confronti del servizio sanitario. Solitamente gli eventi avversi non si verificano mai per una singola causa, ma sono determinati da una serie di variabili che interagendo fra di loro fanno scaturire questi fenomeni come la tipologia di compito, l'equipe, l'organizzazione e l'ambiente di lavoro. Le cadute rientrano all'interno delle tematiche prese in esame dal clinical risk management che ha come obiettivo primario la sicurezza del paziente (patient safety) cioè la garanzia di aver ridotto ai minimi livelli possibili, in base alle conoscenze tecniche e scientifiche attuali, il rischio di danno al paziente. ${ }^{1-28}$

\section{Perchè esiste così tanto interesse per le cadute?}

Il fenomeno caduta accidentale si verifica con una certa frequenza in tutte le realtà ospedaliere italiane.

Le cadute accidentali comportano nella maggior parte dei casi: i) spese impreviste in termini di aumento di giorni di degenza, di utilizzo di esami diagnostici mirati per scongiurare una lesione con, infine, un aumento di spesa sanitaria; ii) danni, più o meno gravi, per chi le subisce con ripercussioni psicologiche (post fall syndrome) e fisiche; iii) richiesta di risarcimento.

In forza del fatto che le cadute accidentali possono essere previste nel $78 \%$ dei casi, è un dovere del personale sanitario mettere in atto dei provvedimenti tali da portare a una considerevole riduzione questo fenomeno.

\section{Dimensioni del problema}

\section{$\%$ cadute nazionali}

Qualunque dato è ampiamente sottostimato in quanto, spesso a livello nazionale, i dati sono ricavati dall'analisi delle schede di denuncia di caduta accidentale che hanno conseguenze per la persona e quindi rappresentano solo la punta dell'iceberg.

Riguardo alle conseguenze delle cadute risulta che il $42 \%$ delle persone cadute non ha riportato 
danni, il 30\% degli assistiti ha subito solo delle lesioni lievi e il restante $28 \%$ ha riportato danni gravi o addirittura la morte. La maggior parte delle cadute si verifica nei giorni feriali però considerando che percentualmente i giorni feriali sono circa $1^{\prime} 82 \%$ e i festivi il $18 \%$, si può pensare che il numero dei pazienti che cade giornalmente in un giorno feriale è di molto inferiore al numero dei degenti che cade invece in un giorno festivo (risorse umane inadeguate?), il momento del giorno in cui si verificano le cadute con più frequenza è la fascia notturna (tra le 21:00 e le 6:00).

A questo proposito si possono ipotizzare tre motivazioni: i) il personale in servizio è ridotto rispetto alle altre ore del giorno; ii) degenti tendono in larga parte a manifestare disorientamento spaziotemporale proprio nelle ore notturne: infatti, aumenta lo stato di allucinazione del paziente soprattutto a causa del fatto che è al di fuori del proprio ambiente domestico; iii) la scarsa illuminazione che spesso caratterizza i reparti di degenza nelle ore notturne.

Seguendo questa logica il numero di pazienti che cadono nelle ore del mattino dovrebbe essere inferiore rispetto al pomeriggio, in realtà non è così, una possibile soluzione a questa incongruenza logica può essere data alla luce di due osservazioni: i) il mattino è il momento in cui è concentrata la maggior attività infermieristica al letto del malato infatti in questa fascia oraria si accompagnano i degenti in bagno li si lava al letto li si mobilizza tutte queste pratiche aumentano il rischio di caduta accidentale della persona assistita; ii) La maggior parte degli ospedali limita le visite dei famigliari ai degenti in un arco temporale che va dalle 12:00 alle 20:00 quindi è facile pensare che in questa fascia temporale la sorveglianza sia intensificata grazie al ruolo indiretto di controllo svolto dai famigliari stessi.

Per quanto riguarda le Unità Operative che vengono maggiormente coinvolte nelle cadute ospedaliere troviamo frequentemente la Neurologia e la Medicina Interna proprio perché in essa affluiscono un moltitudine di patologie molto varie tra di loro e quindi non è sempre facile per gli operatori sanitari valutare se un paziente sia più o meno a rischio di caduta. A questo bisogna aggiungere la rapidità di evoluzione delle patologie che si riscontra in questa realtà più che in tutti gli altri settori sanitari.

\section{Conseguenze delle cadute}

I danni possono essere di tipo fisico, in acuto oppure in cronico (traumi, ferite lacero contuse, fratture) o psicologico come la cosiddetta post fall syndrome caratterizzata da paura di cadere di nuovo, perdita di sicurezza, ansia, depressione, fattori che possono condurre a diminuzione dell'autonomia, aumento della disabilità e, in generale, ad una riduzione anche molto significativa della qualità della vita.

Sono da ritenersi lesioni lievi le abrasioni, le ecchimosi e gli strappi muscolari perché sono seguiti da una prognosi sempre favorevole, si risolvono senza interventi terapeutici mirati e nel giro di pochi giorni. Invece sono definite lesioni gravi tutti quei danni che provocano trattamenti terapeutici mirati e hanno dei tempi di guarigione medio-lunghi e lunghi e che non sempre si concludono con una prognosi favorevole (es. trauma cranico commotivo, fratture e stato comatoso).

Oltre ai danni puramente fisici sul paziente però, le cadute avvenute in un contesto di ricovero possono comportare un aumento della degenza, attività diagnostiche e terapeutiche aggiuntive (per es. esami diagnostici mirati per scongiurare una lesione e all'attuazione di interventi terapeutici mirati per risolvere l'eventuale danno cagionato), e/o eventuali ulteriori ricoveri dopo la dimissione, con un incremento dei costi sociali e sanitari, senza calcolare le spese sostenute per eventuali cause medico legali indette dai pazienti o familiari (una percentuale minima delle cadute).

La metà degli anziani che riporta una frattura di femore non è più in grado di deambulare ed il $20 \%$ muore, per complicanze, entro 6 mesi.

La mortalità legata alla caduta in USA è 36,8 per 100.000 persone, mentre in Canada è addirittura 9,4 per 10.000 persone. In Finlandia la mortalità per le persone di più di 50 aa è 55,4 per l'uomo e 43,1 per la donna su 100.000 persone.

\section{L'esperienza al Maggiore di Novara (AOU Maggiore Carità di Novara)}

Dal maggio 2011 è stato istituito un Gruppo di Lavoro sulle Cadute (GLC), il quale ha lo scopo di sorvegliare e controllare il fenomeno, proponendo e impostando idonei interventi di prevenzione sui vari fattori di rischio di caduta.

Il GLC ha analizzato diversi aspetti che possono condizionale il rischio di caduta producendo un documento informativo nel quale si possono ritrovare informazioni sia su come poter valutare il rischio di caduta sia sugli interventi che gli operatori delle Strutture dell'AOU possono mettere in atto in base alla caratteristiche dell'assistito.

\section{La valutazione del rischio di caduta}

Lo screening del rischio è un metodo efficace per identificare le persone a rischio di caduta ed è parte integrante dei programmi di prevenzione attraverso la valutazione di ciascun assistito e l'attuazione di interventi mirati alla riduzione dei fattori di rischio personali ed ambientali. 


\section{QUANDO valutare il rischio di caduta}

Questa valutazione deve essere effettuata su tutte le persone ammesse in ambienti di cura per acuti di carattere generale, medico, chirurgico, riabilitativo, entro 24 ore e qualora le condizioni dell'assistito si modifichino.

Nella valutazione e rivalutazione del rischio di caduta, sulla base delle indicazioni regionali, sono indicati come fondamentali i seguenti momenti: i) ingresso in reparto entro 24 ore; ii) trasferimento di reparto; iii) dimissione; iv) ad ogni cambiamento dei fattori di rischio; v) ad ogni cambiamento delle condizioni dell'assistito.

\section{COSA valutare per il rischio di caduta}

La prima azione necessaria per la prevenzione delle cadute consiste nell'identificare i possibili fattori di rischio in relazione alle caratteristiche dell'assistito e a quelle dell'ambiente e della struttura che lo ospita in termini di sicurezza, di organizzazione e di adeguatezza del processo assistenziale.

In tal senso $\mathrm{i}$ fattori responsabili delle cadute possono essere suddivisi nelle seguenti due categorie:

Fattori intrinseci relativi alle condizioni di salute dell'assistito, che comprendono sia i dati anagrafici sia la patologia motivo del ricovero, le comorbilità e le terapie farmacologiche (es. sedativi, analgesici, ipotensivanti...).

Fattori estrinseci relativi agli aspetti organizzativi della Struttura di degenza, alle caratteristiche ambientali della struttura e dei presidi sanitari impiegati.

In Tabella 1 sono elencati i fattori di rischio associati alle cadute secondo la suddivisione in fattori estrinseci e intrinseci; in Tabella 2 sono elencati i farmaci associati alle cadute.

\section{Come valutare il rischio di caduta all'interno dell'AOU}

La valutazione del rischio di caduta deve essere effettuata mediante uno strumento standardizzato e la valutazione clinica del professionista, elemento irrinunciabile nella definizione del rischio di caduta in quanto nessuno strumento di valutazione del rischio, da solo, è in grado di identificare tutte le persone a rischio o tutti i fattori di rischio (Tabella 3 ).

La scala di valutazione utilizzata in ambito aziendale è la Conley e deve essere utilizzata sia all'ingresso dell'assistito in ospedale sia per il successivo monitoraggio durante il ricovero.

Tabella 1. Fattori associati alle cadute.

\begin{tabular}{|c|c|}
\hline FATTORI INTRINSECI & FATTORI ESTRINSECI \\
\hline Precedenti cadute & Contenzione \\
\hline Deficit della vista & Calzature di misura non adeguata \\
\hline Ictus & Ausili per la mobilizzazione inadeguati \\
\hline Artrite & \multirow{7}{*}{$\begin{array}{l}\text { Fattori ambientali, fra cui: } \\
\text { Scale non sicure } \\
\text { Vasche da bagno senza maniglie } \\
\text { Scarsa illuminazione }\end{array}$} \\
\hline Ipotensione ortostatica & \\
\hline Patologie acute & \\
\hline Instabilità della marcia & \\
\hline Deficit cognitivo & \\
\hline Incontinenza & \\
\hline Farmaci & \\
\hline
\end{tabular}

Tabella 2. Farmaci associati alle cadute.

\begin{tabular}{|l|l|l|}
\hline \multicolumn{1}{|c|}{$\begin{array}{c}\text { Farmaci con effetto sedativo } \\
\text { sul sistema nervoso centrale }\end{array}$} & \multicolumn{1}{|c|}{$\begin{array}{c}\text { Farmaci che agiscono } \\
\text { sul sistema cardiovascolare }\end{array}$} & \\
\hline $\begin{array}{l}\text { Barbiturici } \\
\text { Ipnotico-sedativi }\end{array}$ & $\begin{array}{l}\text { Diuretici } \\
\text { Antiaritmici }\end{array}$ & Tutti i tipi \\
Antidepressivi triciclici & Antipertensivi & \\
Antipsicotici - neurolettici & Vasodilatatori & \\
Antiparkinson & Cardioattivi glicosidi & \\
Analgesici & & \\
Ansiolitici & & \\
Anticonvulsivi & & \\
\hline
\end{tabular}




\section{I fattori di rischio e gli interventi possibili}

In Tabella 4 si propone un modello che riassume gli interventi possibili rispetto ad ogni specifico fattore di rischio.

In caso di valutazione positiva per rischio di caduta, la documentazione clinica deve contenere anche evidenza degli interventi messi in atto per ridurre tale rischio.

\section{Nota importante}

In ogni riga sono stati evidenziati gli interventi direttamente collegati al singolo fattore di rischio, ma occorre sempre valutare l'aggiunta di altri interventi. Ad esempio, per il fattore precedenti cadute sono stati presi in considerazione $i$ soli interventi legati alle precedenti cadute e non alle singole cause che potrebbero averle originate; in caso di precedenti cadute da ipotensione ortostatica, si consiglierebbe quindi di attivare gli interventi per precedenti cadute e quelli per ipotensione ortostatica.

\section{Informazione ed educazione} a assistito/familiari/caregivers

- Informare e istruire l'assistito e i familiari con informazioni adeguate alla loro comprensione.

- Illustrare l'ambiente circostante all'assistito e a chi lo segue; all'ingresso in struttura mostrare: i) il bagno, la stanza di degenza e altre eventuali parti importanti della Struttura; ii) l'uso dei sistemi di chiamata in caso di bisogno (campanello). Sincerarsi che il sistema sia funzionante e facilmente raggiungibile dall'assistito; iii) ose disponibile, la presenza di ausili e supporti (es. corrimano nei corridoi, maniglie nei servizi igienici, ...).

- Richiedere la collaborazione di persone significative per l'assistito e favorire la presenza dei familiari.

- Soprattutto in caso di alto rischio di caduta, spiegare all'assistito e a chi lo segue che è importante avvisare gli Operatori del reparto se si reca in bagno o si allontana dalla stanza di degenza.

- In caso di rischio di caduta, informare l'assistito e chi lo segue sui fattori di rischio presenti; a tale scopo avvalersi anche di materiale informativo cartaceo.

L'AOU mette a disposizione un opuscolo informativo scaricabile in formato pdf dalle pagine aziendali del DiPSa (Direzione Professioni sanitarie). La consegna dell'opuscolo va accompagnata da informazioni verbali.

Tabella 3. Come valutare il rischio di caduta all'interno dell'AOU.

\begin{tabular}{|c|c|}
\hline $\begin{array}{l}\text { 1. Eseguire la valutazione } \\
\text { del rischio di caduta in } \\
\text { tutti gli assistiti ricoverati } \\
\text { in AOU }\end{array}$ & $\begin{array}{l}\text { Momenti in cui eseguire la valutazione. } \\
\text { - Ingresso in reparto entro } 24 \text { ore } \\
\text { - Ad ogni cambiamento dei fattori di rischio, compresa la variazione della terapia } \\
\text { - in caso di introduzione di farmaci } \\
\text { - Ad ogni cambiamento delle condizioni dell'assistito } \\
\text { - Trasferimento di reparto / Dimissione }\end{array}$ \\
\hline $\begin{array}{l}\text { 2. Eseguire la valutazione } \\
\text { del rischio di caduta con } \\
\text { uno strumento } \\
\text { standardizzato } \\
\text { (scala di Conley) }\end{array}$ & $\begin{array}{l}\text { La scala di Conley è stata individuata dall'AOU come strumento di predizione del rischio } \\
\text { di cadute accidentali ed è compresa fra quelle elencate nelle linee di indirizzo regionali. } \\
\text { Pur essendo testata sulla popolazione con età maggiore di } 50 \text { anni, la scala va } \\
\text { utilizzata per tutti i ricoverati in quanto indaga i principali fattori di rischio per caduta. } \\
\text { Si ribadisce comunque che la scala di Conley (come ogni test) rappresenta un indirizzo } \\
\text { per l'équipe assistenziale, che deciderà di adottare misure preventive tenendo conto non } \\
\text { solo del risultato della scala, ma anche della valutazione clinica globale dell'assistito. A } \\
\text { tale proposito il professionista sanitario cercherà anche la presenza di altro fattori } \\
\text { fortemente correlati al rischio di caduta come: } \\
\checkmark \text { presenza di più di } 4 \text { farmaci in terapia } \\
\checkmark \text { assunzione di farmaci sedativi o ipnotici } \\
\checkmark \text { incontinenza o necessità di usare frequentemente la toilette } \\
\checkmark \text { incapacità a mantenere la posizione eretta o seduta in autonomia } \\
\checkmark \text { deambulazione con ausili come stampelle, bastone o deambulatore }\end{array}$ \\
\hline $\begin{array}{l}\text { 3. Conservare l'evidenza } \\
\text { della valutazione del } \\
\text { rischio di caduta nella } \\
\text { documentazione clinica }\end{array}$ & \\
\hline
\end{tabular}




\section{Formazione degli operatori}

Il personale sanitario che lavora con assistiti a rischio di caduta deve sviluppare e mantenere competenze di base nella valutazione e nella prevenzione delle cadute. Le competenze sono sviluppabili attraverso la partecipazione ad eventi formativi specifici e la discussione dell'argomento all'interno del gruppo assistenziale (Figure 1 e 2).

\section{Contenzione fisica}

Il ricorso alla contenzione è da valutare con molta attenzione soprattutto in ordine agli aspetti emersi dall'esame della letteratura.

L'indebolimento muscolare e la riduzione della funzionalità fisica sono riconosciuti come fattori di rischio per le cadute ed entrambi questi fattori costituiscono un effetto secondario alla limitazione della libertà di movimento.

L'uso della contenzione fisica in ambito ospedaliero ha mostrato correlazioni con l'aumento di rischio di caduta, di lesioni gravi e di prolungamento dell'ospedalizzazione.

È bene valutare attentamente l'uso di misure di contenzione in assistiti che presentino uno stato confusionale o limitazioni cognitive, in quanto tali misure possono determinare un peggioramento del quadro clinico e aumentare potenzialmente il rischio di caduta.

Da ultimo si ricorda che la contenzione fisica è oggetto di attenzione da parte della normativa sia civile sia penale; il Codice di Deontologia Infermieristica ha un articolo a riguardo (art. 30).

\section{Documentazione clinica sul rischio di caduta}

La valutazione del rischio di caduta va attuata a livello interdisciplinare fra i professionisti coinvolti nel processo di assistenza, diagnosi e cura e gli interventi messi in atto devono essere documentati dagli operatori (in particolare medico e infermiere).

Una documentazione infermieristica conforme al modello approvato dal DiPSa contiene gli strumenti per stimare il rischio di caduta, programmare e attuare molti degli interventi suggeriti, valutare il risultato finale del processo assistenziale.

Se la SC non dispone di una documentazione conforme al modello approvato dal DiPSa per la parte infermieristica, deve comunque certificare in forma scritta l'avvenuta valutazione del rischio, gli eventuali interventi attuati e le successive rivalutazioni.

L'utilizzo di documentazione per valutare il rischio di caduta e i conseguenti interventi sono oggetto di verifica da parte del GLC.

Tabella 4. Interventi possibili rispetto ad ogni specifico fattore di rischio.

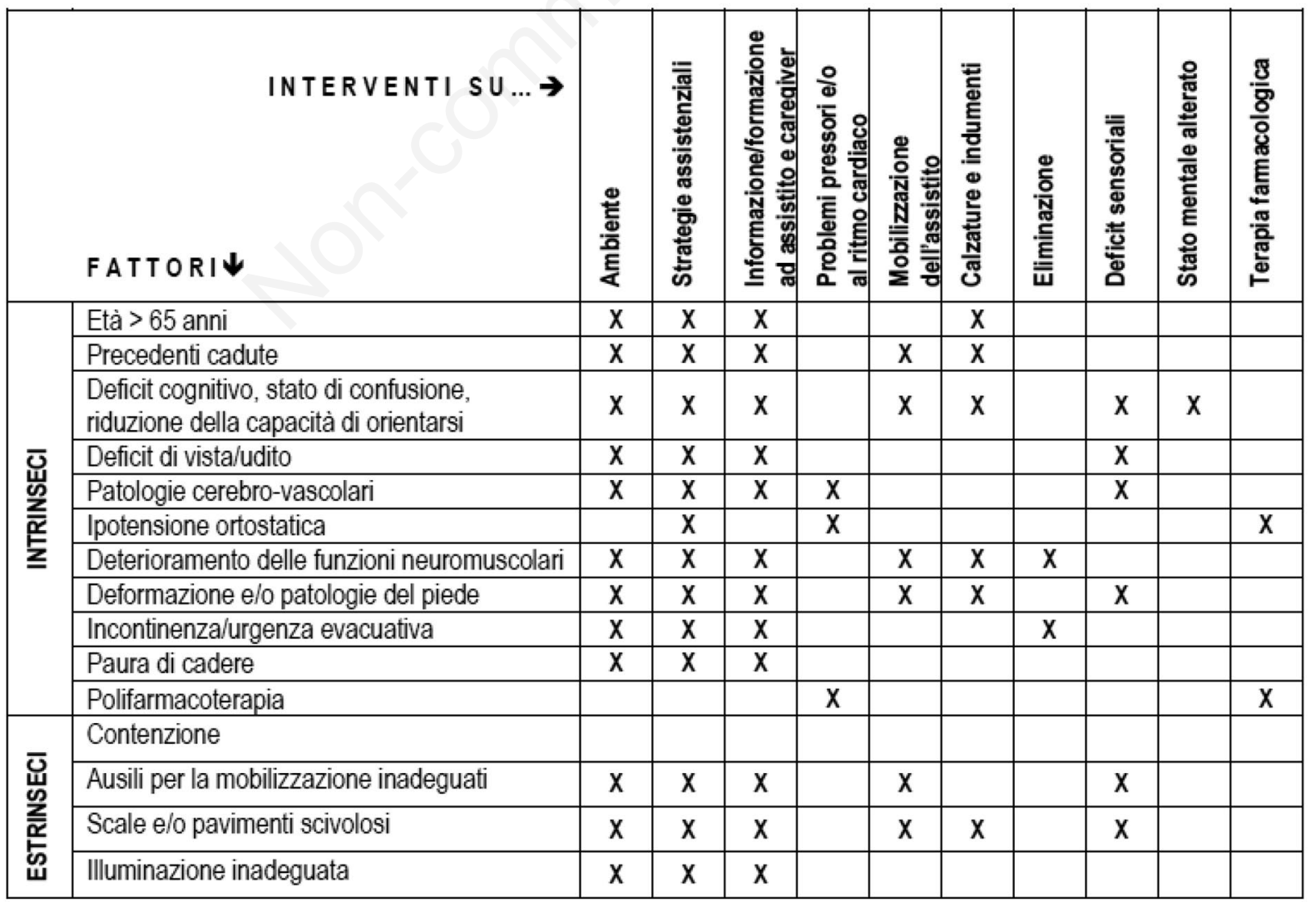




\section{Modulo di segnalazione delle cadute}

Per segnalare l'avvenuta caduta del degente viene utilizzato apposito modulo compilato da infermiere e medico in servizio al momento della caduta, il modulo è di semplice compilazione, completo negli aspetti anamnestici, farmacologici, di specifica sul luogo della cauta e sui procedimenti intrapresi. Tali moduli devono essere inviati dal coordinatore infermieristico che li firma per presa visione (Figura 3).

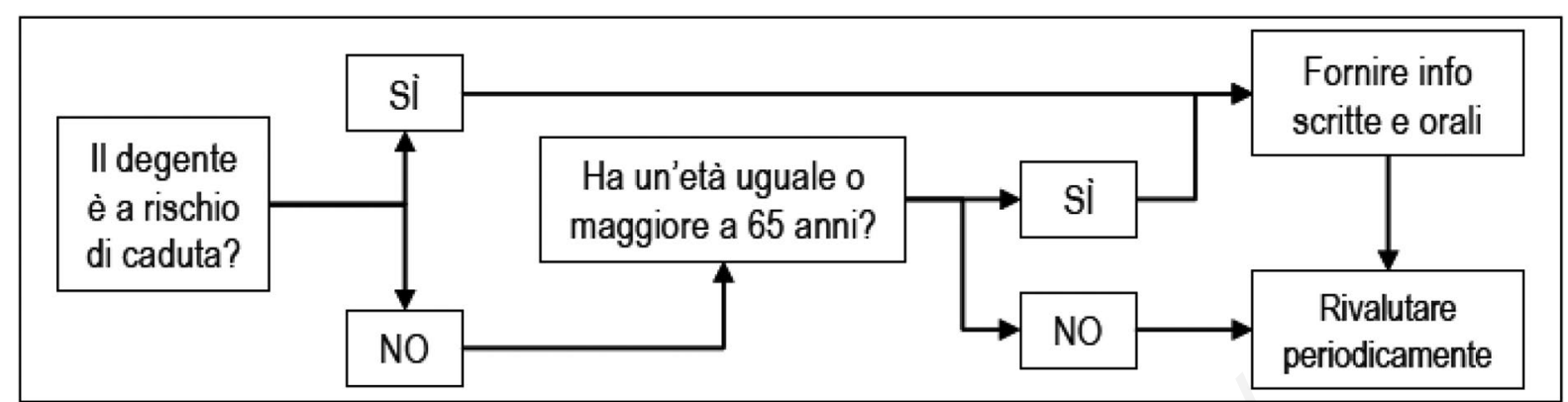

Figura 1. Flusso decisionale per fornire informazioni sul rischio di caduta.

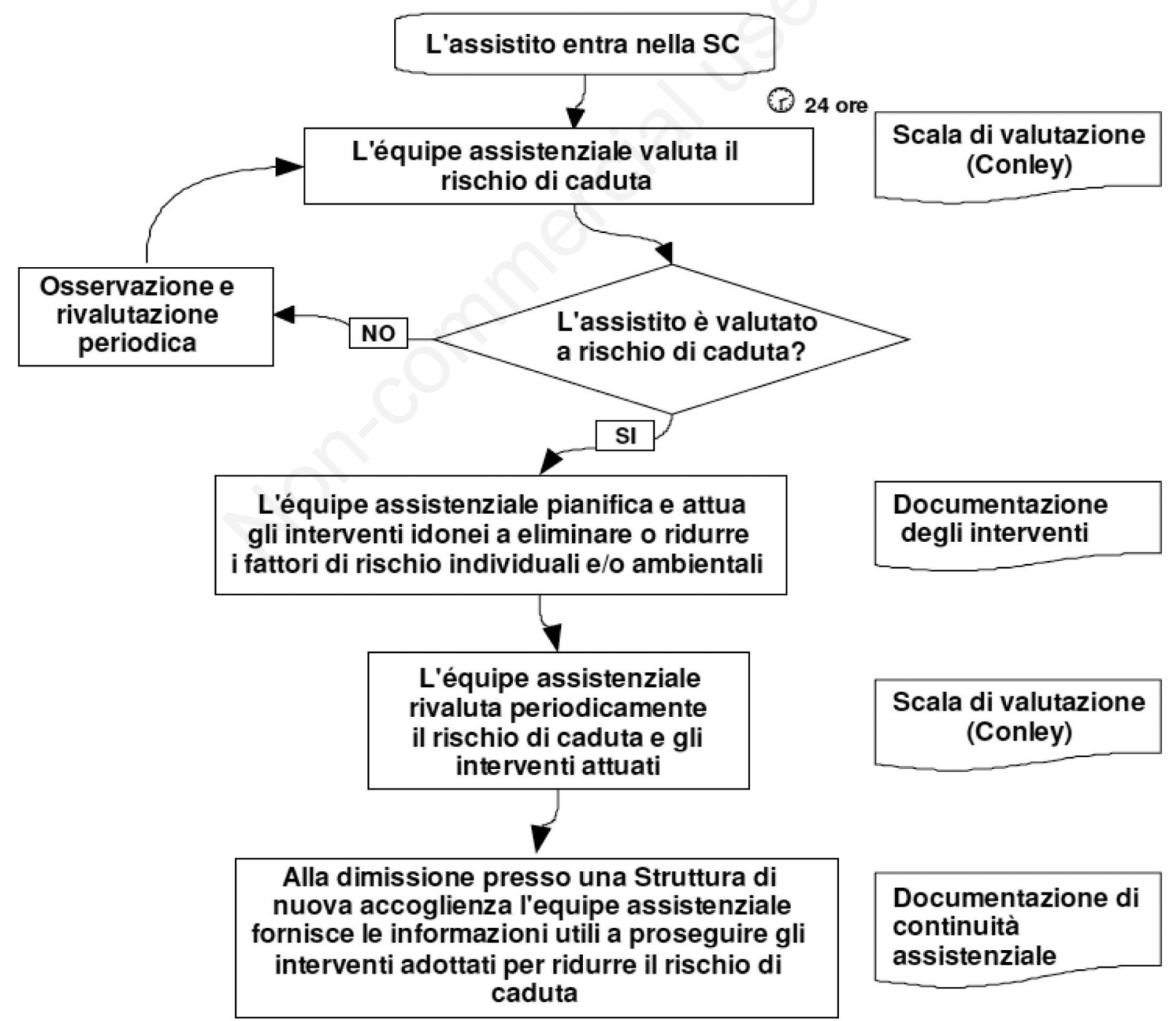

Figura 2. Flow chart per la prevenzione delle cadute. 


\begin{tabular}{|c|}
\hline MO_DMP_001 \\
\hline $\begin{array}{c}\text { "SCHEDA SEGNALAZIONE } \\
\text { CADUTA" }\end{array}$ \\
\hline
\end{tabular}

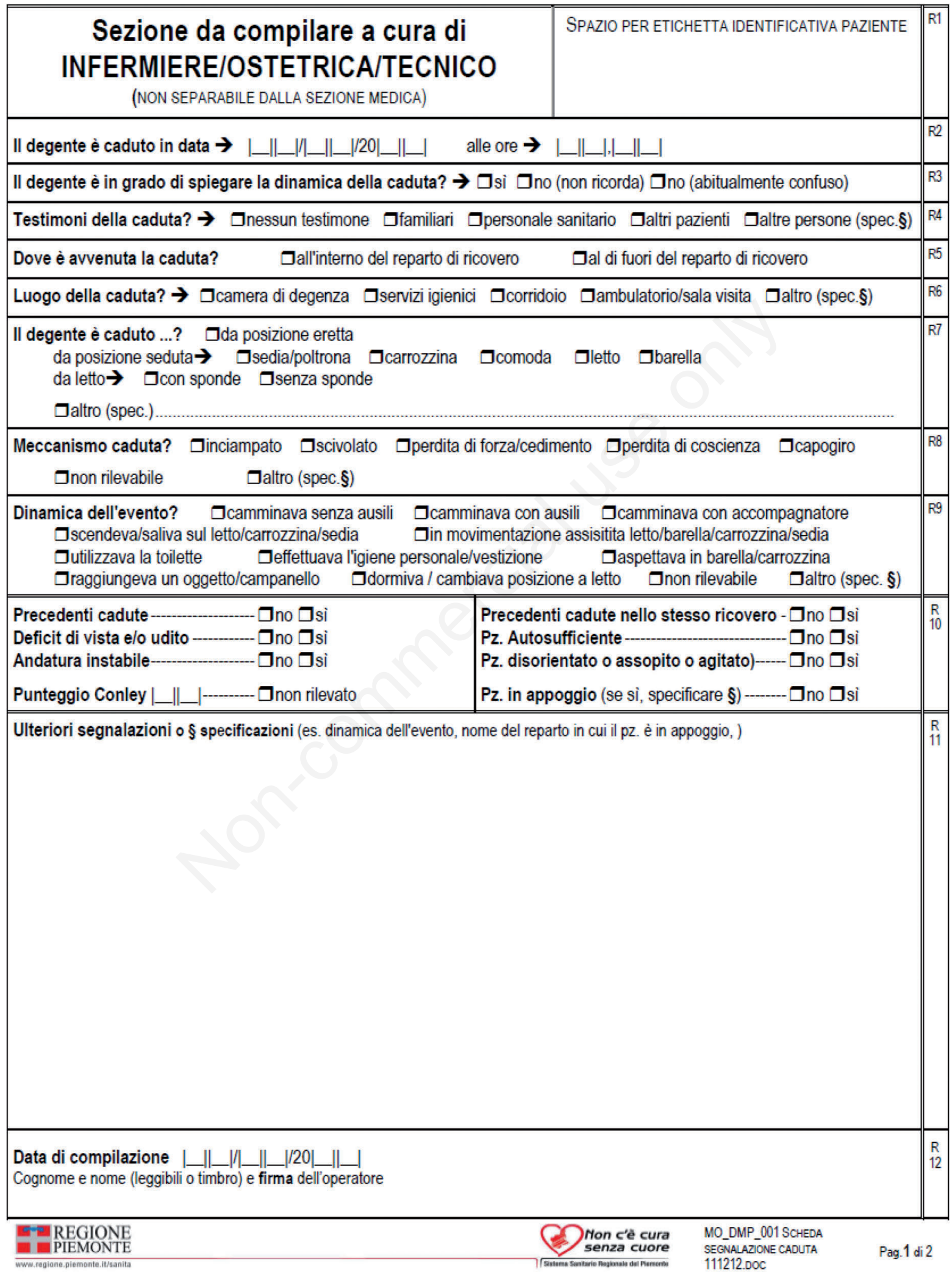

Figura 3. Modulo segnalazione cadute. Lato A. 


\begin{tabular}{|l|l|l|}
\hline Sezione da compilare a cura del MEDICO & SPAZIO PER ETICHETTA IDENTIFICATIVA PAZIENTE & R1 \\
\hline
\end{tabular}

(NON SEPARABILE DALLA SEZIONE INFERMIERISTICA/OSTETRICA/TECNICA)

Conseguenze della caduta $\rightarrow \square$ senza danno $\square$ con danno $\rightarrow$ per descrivere il danno compilare la/e descrizione/i della/e sede/i e i danni riportati; in casi particolari (es. politraumatismi) o in presenza di più di due sedi utilizzare lo spazio delle ulteriori segnalazioni

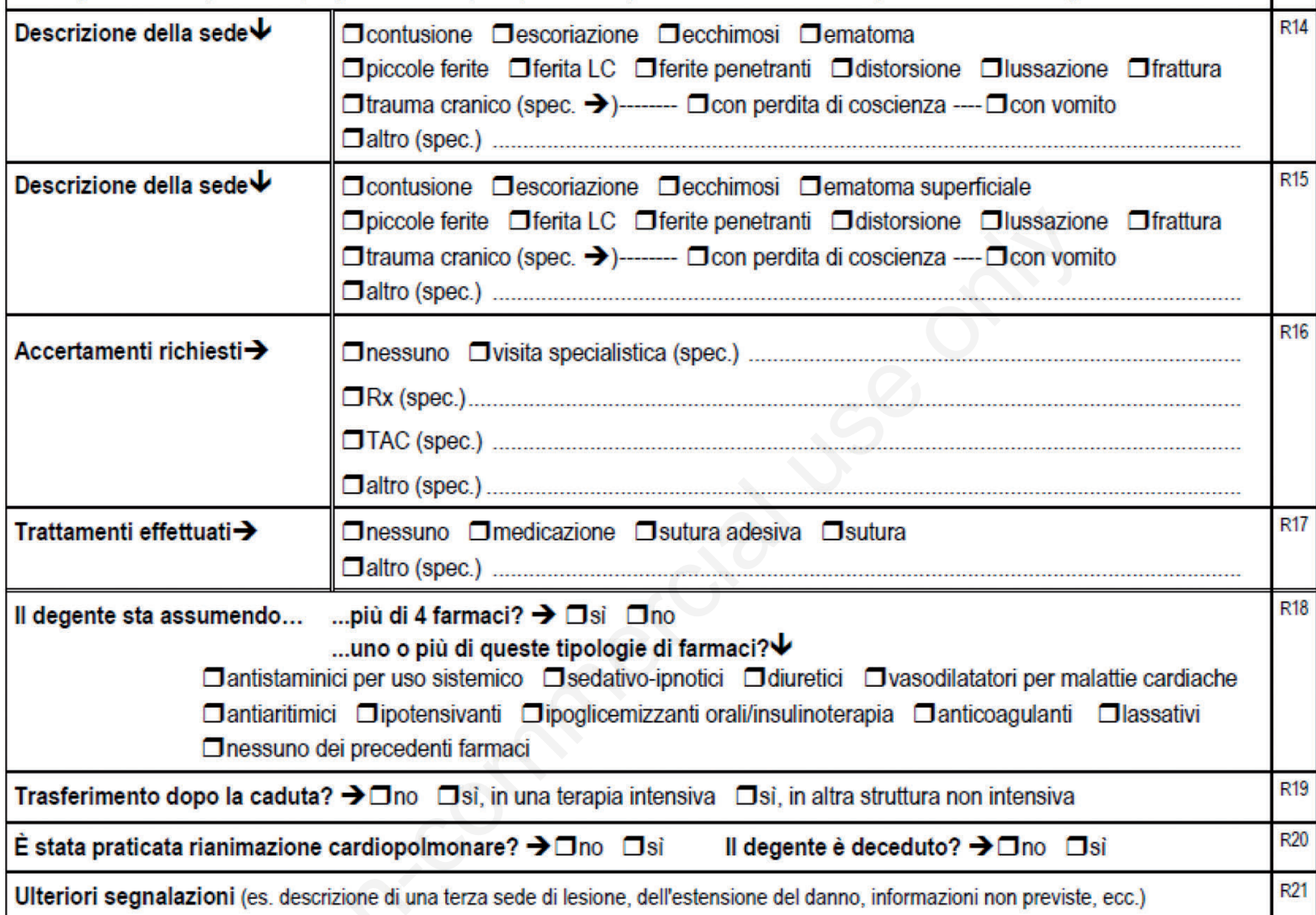

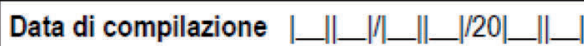

Cognome e nome (leggibili o timbro) e firma del Medico

\begin{tabular}{|c|c|c|}
\hline 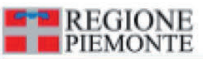 & $\begin{array}{l}\text { Mon c'è cura } \\
\text { senza cuore }\end{array}$ & \\
\hline Www. regione. piemonte. Ittsanita & 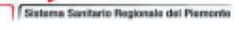 & $\begin{array}{l}\text { SEGNALAZIONE CADUTA } \\
111212.0 \circ \mathrm{C}\end{array}$ \\
\hline
\end{tabular}

Figura 3. Modulo segnalazione cadute. Lato B. 


\section{Bibliografia}

1. Alarcon T, Gonzalez-Montalvo J.I. , Barcena A. "Postfall syndrome: a matter to study in patients with hip fractures admitted to orthopaedic wards". Age Ageing 2006; 35 (2): 205-206

2. Bendanti D, Nanni I. "Attività di ricerca per il miglioramento assistenziale, con finalità di erogare le prestazioni in base alle prove di efficacia; prevenzione delle cadute dei pazienti". Documento finale del programma di formazione sul campo per la prevenzione delle cadute nei pazienti presso Servizio Sanitario Regionale Emilia-Romagna, dicembre 2006.

3. Burato E, "La gestione del rischio ed il miglioramento della qualità in Sanità". Dispensa master in coordinamento delle professioni infermieristiche ed ostetriche coops 11, 2009: 5-6; 13-19.

4. Caldara C. Destrebecq A. and Savoldi L. "Determinazione del valore predittivo di una scala di valutazione del rischio di cadute in pazienti anziani ospedalizzati". Assistenza infermieristica e ricerca, 2008: 27 (3) 151-155.

5. Chiari P., Mosci D., Sabrina Fontana S. "Valutazione degli strumenti di misura del rischio di cadute dei pazienti”. Assistenza Infermieristica e Ricerca, 2001: 21 (3) :117-124

6. Chiari P. "Suggerimenti di pratica clinica per la prevenzione delle cadute dei pazienti in ospedale ". Azienda Ospedaliera di Bologna Policlinico S. Orsola Malpighi, Centro Studi EBN, dicembre 2004

7. Conley D. Schultz AA, Selvin R. "The challenge of predicting patients at risk for falling: development of the Conley Scale". Med surg Nurs 1999; 8: 348-54

8. Del Poeta G. Mazzufero F, Canepa M. "Il risk management nella logica del governo clinico". McGraw Hill, 2006

9. Dykes P,. Carroll D, McColgan K. "Scales for assessing self-efficacy of nurses and assistants per preventing falls". Journal of advanced Nursing 2010; 67(2); 438449

10. Gaebler S.” Predicting which patient will fall again and again". Journal of Advanced Nursing, 1993; 18 :18951902

11. Gruppo di lavoro regionale per la prevenzione del rischio cadute Regione Lombardia - "La gestione del rischio di cadute in pazienti ospedalizzati e ospiti di RSA/RSD a gestione diretta". Percorso formativo IREF Milano - Maggio 2010

12. Heinze C. Dassen T, Grittner U." Use of physical restraints in nursing homes and hospitals and related factors: a cross-sectional study". J Clin Nurs 2012; 21(78): 1033-40

13. Helden V. S, Wyers C. E., Dagnelie P.C. "Risk of falling in patients with a recent fracture". BMC Musculoskelet
Disord. 2007; 8: 55

14. Joint Commission International: "Standard per gli Ospedali e per la sicurezza del paziente". Progetto per il servizio triennale di valutazione delle aziende sanitarie accreditate e di trasferimento del know-how alle Aziende Sanitarie Locali 2008.

15. Morse JM, Black C, Oberle K, Donahue P. "A prospective study to identify the fall prone patient". Soc Sci Med 1989; 28(1): 81-6

16. Morse JM. "Preventing Patient Falls". Int J Nurs Prac 1997; 9(4):223-35

17. Morse JM. "Enhancing the safety of hospitalization by reducing patient falls" Am. J. Infect. Control 2002; 30 (6): $376-80$

18. Ministero della Salute " Protocollo per il Monitoraggio degli Eventi Sentinella" 2009.

19. National Institute for Health and Clinical Excellence. “ NICE Guidelines". Clinical Practice Guideline for the assessment and prevention of falls in older people. 2004

20. Oliver D. Britton M, Seed P, "Development and evaluation of evidence based risk assessment tool (STRATIFY) to predict which elderly inpatients will fall: case-control and cohort studies". BMJ 1997; 315 (7115):1049-53

21. Oliver D. Connelly JB, Victor CR. "Strategies to prevent falls and fractures in hospitals and care homes and effect of cognitive impairment: systematic review and metaanalyses".BMJ 2007 Jan 13; 334(7584):82

22. Programma nazionale per le Linee Guida- PNLG Prevenzione delle cadute da incidenti domestico negli anziani - aggiornamento maggio 2009

23. RACCOMANDAZIONE $\mathrm{N}^{\circ} 13$ MINISTERO SALUTE "Raccomandazione per la prevenzione e la gestione della caduta del paziente nelle strutture sanitarie" novembre 2011.

24. Regione Lombardia, Direzione Generale Sanità . "Linee di indirizzo e requisiti minimi regionali per l'implementazione di un sistema per la prevenzione e la gestione del rischio caduta del paziente degente in ospedale o in RSA-RSD”. Atto identificativo n ${ }^{\circ} 625$ del 22.7.2010

25. Tinetti Me. Speechley M. "Prevention of falls among the elderly”. N Engl J Med. 1989 Apr 20; 320 (16): 1055-1059

26. Trioni G. "Implementazione di un progetto di risk management: la prevenzione delle cadute accidentali". Management Infermieristico, Vesalio edizioni n 4; 2007.

27. Vanzetta M. Vallicella F. "Qualità dell'assistenza, indicatori, eventi sentinella: le cadute accidentali in ospedale". Management Infermieristico 2001; 2 : 32-36.

28. Zappa P. " La prevenzione dei danni al paziente in seguito a cadute in ospedal " Corso di aggiornamento accreditato ECM/CPD Cantù 11 Maggio 2010 Menaggio, 19 maggio 2010. 


\title{
Protezione dalle infezioni correlate all'assistenza
}

\author{
Ada Maffettone, Carmela Peirce, Sergio Di Fraia \\ UOC Medicina ad Indirizzo Cardiovascolare e Dismetabolico, AO Specialistica dei Colli, Napoli, Italia
}

\section{Cenni storici}

Nella seconda metà del XIX secolo i dottori I. Semmelweiss e J. Simpson furono i pionieri di una raccolta sistematica dei tassi di infezione ospedaliera. ${ }^{1}$

Nel 1846 Semmelweiss (Figura 1) dimostrò che l'ospedale potesse rappresentare un ambiente capace di generare patologie infettive e che tali infezioni potessero essere prevenibili. Durante il periodo in cui esercitava la professione nella clinica ginecologica di Vienna, comprese che l'altissima mortalità per febbre puerperale che si registrava tra le partorienti era dovuta ad una infezione trasmessa alle stesse dalle mani dei medici e degli studenti di medicina che, dalla sala dove praticavano le autopsie, si recavano poi a visitare le gestanti o le puerpere. La sua era un'osservazione empirica ma giusta: a quel tempo infatti medici e studenti non usavano i guanti e passavano dalla sala delle autopsie alla sala parto senza mai lavarsi le mani. Egli comprese che tale evento potesse essere prevenibile: bastò che imponesse agli studenti una scrupolosa pulizia delle mani e la disinfezione con un antisettico (cloruro di calcio) per far crollare di colpo l'indice di mortalità dovuto a febbre puerperale, nella Clinica da lui diretta, dal $12,2 \%$ allo $0,5 \%$. Semmelweiss fu il primo a riconoscere quindi, grazie alle sue brillanti intuizioni e supposizioni, la presenza delle infezioni ospedaliere (I.O.): sin da quel tempo tentò di introdurre quella che è una delle pratiche ancora oggi più efficaci per evitare tali infezioni: il lavaggio delle mani. Fu però ferocemente osteggiato dalla quasi totalità dei suoi colleghi e neanche la pubblicazione dell'opera Etiologia, concetti e profilassi della febbre

Corrispondente: Ada Maffettone, UOC Medicina ad Indirizzo Cardiovascolare e Dismetabolico, AO Specialistica dei Colli, Napoli, Italia.

E-mail: adamaff@hotmail.com

Articolo pubblicato secondo la Creative Commons Attribution NonCommercial 4.0 License (CC BY-NC 4.0).

${ }^{\circ}$ Copyright: the Author(s), 2019

Licensee PAGEPress, Italy

QUADERNI - Italian Journal of Medicine 2019; 7(6):85-90 puerperale (Figura 2) riuscì ad evitare la morte delle puerpere per mancanza di un'accurata asepsi peri partum negli anni a venire.

Il chirurgo J. Simpson, inoltre, nel 1860 evidenziò una correlazione diretta tra mortalità per infezione, dopo amputazione degli arti, e grandezza dell'ospedale (condizione associata al sovraffollamento); il sovraffollamento, infatti, rappresentava per Simpson un fattore favorente la trasmissione delle infezioni da paziente a paziente.

J. Lister, inoltre, sostenne la teoria che l'aria rappresentasse la principale fonte di infezione; negli ospedali grandi e compatti era presente un maggior numero di infezioni rispetto alle cliniche più piccole. L'acido fenico fu utilizzato come primo disinfettante; furono utilizzati anche alcuni nebulizzatori che diffondevano dell'acido fenico attorno al tavolo operatorio al fine di creare un ambiente asettico.

Tra la fine dell' 800 e l'inizio del ' 900 , la scoperta dei microrganismi ha permesso di orientare gli studi principalmente sui germi e quindi di puntualizzare misure preventive atte a ridurre la trasmissione degli stessi al paziente. In quegli anni si dimostrò l'importanza di usare la mascherina al fine di contenere l'immissione di microrganismi nell'ambiente: l'introduzione di mascherine, guanti e camici in sala operatoria cominciò proprio all'inizio del XX secolo. L'applicazione di tecniche asettiche, inoltre, permise di porre sotto controllo la diffusione delle infezioni in ospedale. L'introduzione sul mercato degli antibiotici durante la $2^{\circ}$ Guerra Mondiale contribuì a diffondere l'idea che l'antibiotico potesse sradicare qualsiasi infezione e microrganismo. L'impulso decisivo per l'istituzione di una sorveglianza sanitaria e di una epidemiologia ospedaliera fu dato da una pandemia causata da Staphilococcus aureus meticillino-resistente in ospedali inglesi e statunitensi. Alla fine degli anni ‘ 50 , quindi, Moore istituì la figura professionale dell'infermiera addetta al controllo delle infezioni nel suo ospedale, esempio seguito successivamente da altri.

Negli anni '70 si iniziò a delineare una popolazione di pazienti ad aumentato rischio per infezioni ospedaliere (IO), ad esempio quelli sottoposti a pratiche invasive durante la degenza.

Gli Stati Uniti, così, istituirono i Center for Disease Control and Prevention (CDC) e la Divisione Infezioni 
Ospedaliere e svilupparono le definizioni standard mediante l'avvio di un sistema di sorveglianza attiva, il cosiddetto National Nosocomial Infections Survillance System (NNIS), ancora oggi attivo., ${ }^{2,3}$

\section{Sistema di regolamentazione in Italia del Piano Sanitario Nazionale}

In Italia, negli anni '80, l'Istituto Superiore di Sanità (ISS) elabora il programma di controllo delle IO (SIPIO/1984 - Studio Italiano Prevenzione Infezioni Ospedaliere), mediante l'emanazione di due circolari del Ministero: $N^{\circ} 52 / 1985$ e N ${ }^{\circ} 8 / 1988 .{ }^{4}$ Esse hanno rappresentato una vera e propria lotta contro le IO, mediante la presa di coscienza delle principali cause di IO: i) la selezione dei ceppi batterici antibiotico-resistenti; ii) l'inefficacia degli agenti antivirali; iii) la mancata disponibilità di nuovi vaccini; iv) la maggiore tendenza all'ospedalizzazione; v) il maggiore utilizzo in terapia di sangue e derivati.

Si comprendeva, infatti, che l'azione di gran lunga più efficace nel controllo delle infezioni ospedaliere fosse rappresentata dalla sorveglianza mirata, intesa come la raccolta continua di informazioni, analisi dei dati, applicazione di misure di controllo e valutazione dell'efficacia delle stesse. I sistemi di sorveglianza continua si sono infatti dimostrati molto efficaci, se orientati a specifiche aree di rischio, mentre un unico schema di sorveglianza applicato a tutto l'ambiente ospedaliero si è dimostrato meno efficace. ${ }^{5,6}$

Nel 1990, in Italia, vengono emanati i Decreti Legislativi 502/92 e 517/93 per la riorganizzazione delle strutture ospedaliere. ${ }^{7}$ Il Piano Sanitario Nazionale tra il 2002 ed il 2004 identifica le infezioni correlate all'assistenza (ICA) come errori nelle pratiche assistenziali, quindi prevenibili.

\section{Le infezioni correlate all'assistenza}

Per ICA si definiscono le infezioni acquisibili in ospedale come in comunità, ma correlate al processo assistenziale attuato, cioè infezioni contratte durante il ricovero ospedaliero, che si manifestano clinicamente almeno dopo 48 ore dall'ingresso del ricovero, durante la degenza stessa o dopo la dimissione. Le infezioni acquisite in ospedale comprendono anche le infezioni che il personale ospedaliero può contrarre nell'assistenza ai malati. L'infezione va distinta dalla colonizzazione, definita come la moltiplicazione a livello locale di microrganismi senza apparenti reazioni tessutali o sintomi clinici.

Il serbatoio di questi microrganismi (habitat naturale e sede di moltiplicazione) è costituito dall'uomo (pazienti infetti/colonizzati e/o portatori) e dall'ambiente.

Affinché avvenga la trasmissione del micro organismo, è necessario che vi sia un veicolo. Un veicolo di importanza determinante è costituito dalle mani degli operatori sanitari, sulle quali colonizzano un numero e una varietà non indifferente di potenziali patogeni. L'importanza di tale veicolo è sottolineata dal fatto che le misure di controllo più efficaci della maggior parte delle infezioni ospedaliere si basano sul lavaggio frequente delle mani.

Le infezioni ospedaliere relativamente alla provenienza dell'agente eziologico, si distinguono in: i) endogene quando l'infezione è sostenuta da un agente

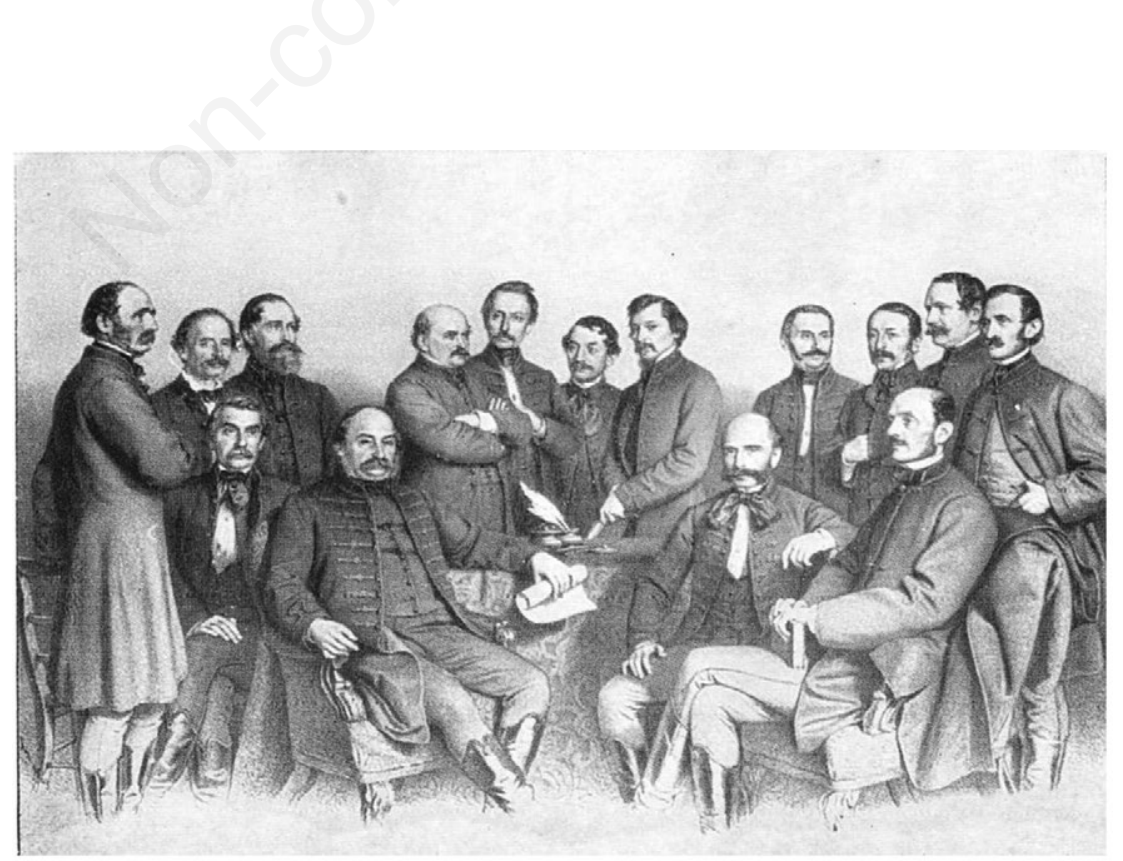

Figura 1. Professori della Facoltà di Medicina di Pest, 1863. Semmelweis è il quarto in piedi da sinistra con le braccia incrociate. 
già da tempo presente nell'organismo del soggetto in causa, in veste di ospite abituale non patogeno, ma che ha acquistato patogenicità e virulenza in seguito ad una compromissione delle difese dell'organismo; ii) esogene le infezioni in cui il germe arriva al paziente trasmesso da un altro paziente (infezione crociata) o dall'ambiente ospedaliero, secondo contatto diretto oppure contatto indiretto, in cui il patogeno è trasportato dalla fonte di infezione all'ospite recettivo da un veicolo animato o inanimato (per esempio endoscopi, strumenti chirurgici, mani ...).

Risulta importante, quindi, considerare fattori intriseci (associati alle condizioni del paziente) e fattori estrinseci (associati a procedure invasive e assistenziali, alla mancata adozione di misure di prevenzione, associati a caratteristiche organizzative dell'ospedale).

Oggigiorno, le ICA sono insite nell'attività sanitaria ed il loro azzeramento risulta impossibile. Esse rappresentano una priorità assoluta per la sanità pubblica e la loro riduzione è correlata alla valutazione del Risk Management.

Le infezioni ospedaliere si manifestano con alti tassi di morbidità ed incremento della mortalità, contribuendo anche all'aumento dei costi di degenza, dei pazienti ospedalizzati.

Nonostante le ampie conoscenze disponibili in materia, sia in termini di fattori di rischio, sia di metodi per la prevenzione, la frequenza delle infezioni ospedaliere non risulta essere in declino.

In Europa, 37 mila persone muoiono per le infezioni ospedaliere. In Italia, 500 mila pazienti sviluppano infezioni correlate all'assistenza ospedaliera, causando circa 2000 decessi con un costo di 1 miliardo di euro.

Le cause, secondo l'Organizzazione Mondiale

\section{IGNAZ SEMMELWEIS}

The ETIOLOGY, CONCEPT, and PROPHYLAXIS of CHILDBED FEVER

\section{Translated by \\ K. CODELL CARTER}

Figura 2. Etiologia, concetti e profilassi della febbre puerperale. della Sanità (OMS) sono per lo più legate: i) all'antibioticoterapia; ii) all'incremento delle tecniche invasive; iii) all'età avanzata; iv) alla scarsa educazione sanitaria del personale; v) alla inadeguatezza delle strutture architettoniche; vi) all'elevato numero di persone che assiste il paziente.

Nel 25-30\% dei casi le ICA possono essere prevenute mediante l'attuazione di una buona pratica clinica, l'utilizzo di appropriate tecnologie e l'inserimento di meccanismi di controllo. I dati Marsh (2004-2011) mostrano che si arriva ad un totale di risarcimenti annui pari a circa 8 milioni per i sinistri nella sanità pubblica.

L'era post-antibiotici, nella quale infezioni comuni e lievi ferite possono diventare mortali, non è una fantasia apocalittica, ma una reale possibilità del XXI secolo. Il flop degli antibiotici causerà una vera e propria epidemia di resistenza che potrebbe colpire, da qui a 20 anni, 200 mila cittadini del Regno Unito, di cui almeno 80 mila moriranno.

\section{Fattori di rischio}

Le persone a rischio di contrarre un'ICA sono innanzitutto i pazienti e, con minore frequenza, il personale ospedaliero, gli assistenti volontari, studenti e tirocinanti. Tra le condizioni che aumentano la suscettibilità alle infezioni ci sono: i) età (neonati, anziani); ii) altre infezioni o gravi patologie concomitanti (tumori, immunodeficienza, diabete, anemia, cardiopatie, insufficienza renale); iii) malnutrizione; iv) traumi, ustioni; v) alterazioni dello stato di coscienza; vi) trapianti d'organo.

\section{Principali meccanismi di trasmissione delle ICA}

I principali meccanismi di trasmissione delle ICA sono: i) contatto diretto tra una persona sana e una infetta, soprattutto tramite le mani; ii) contatto tramite le goccioline emesse nell'atto del tossire o starnutire da una persona infetta a una suscettibile che si trovi a meno di $50 \mathrm{~cm}$ di distanza; iii) contatto indiretto attraverso un veicolo contaminato (per esempio endoscopi o strumenti chirurgici); iv) trasmissione dell'infezione a più persone contemporaneamente, attraverso un veicolo comune contaminato (cibo, sangue, liquidi di infusione, disinfettanti, ecc.); v) via aerea, attraverso microrganismi che sopravvivono nell'aria e vengono trasmessi a distanza.

\section{Tipologia delle infezioni}

Circa $1 ' 80 \%$ di tutte le infezioni ospedaliere riguarda quattro sedi principali: i) il tratto urinario; ii) 
le ferite chirurgiche; iii) l'apparato respiratorio; iv) le infezioni sistemiche (sepsi, batteriemie).

Le più frequenti sono le infezioni urinarie, che da sole rappresentano il $35-40 \%$ di tutte le infezioni ospedaliere. Tuttavia, negli ultimi quindici anni si sta assistendo a un calo di questo tipo di infezioni (insieme a quelle della ferita chirurgica) e ad un aumento delle batteriemie e delle polmoniti. L'aumento delle infezioni sistemiche è la conseguenza di un graduale aumento dei fattori di rischio specifici quali l'uso eccessivo di antibiotici e di cateterismi vascolari.

Per quanto riguarda i microrganismi coinvolti, variano nel tempo. Fino all'inizio degli anni Ottanta, le infezioni ospedaliere erano dovute principalmente a batteri gram-negativi (per esempio, Escherichia coli e Klebsiella pneumoniae). Poi, per effetto della pressione antibiotica e del maggiore utilizzo di presidi sanitari di materiale plastico, sono aumentate le infezioni sostenute da Gram-positivi (soprattutto Enterococchi e Staphilococcus epidermidis) e quelle da miceti (soprattutto Candida), mentre sono diminuite quelle sostenute da Gram-negativi.

Attualmente: i) la $K$. pneumoniae presenta resistenza alla $3^{\circ}$ generazione di cefalosporine e ai carbapenemi tra il 25 e il $50 \%$ (in Europa 8,3\%); ii) l'E. coli, Acinobacter e $S$. aureus resistente alla meticillina hanno percentuali di resistenza fra il $25 \%$ e il $50 \%$. I siti maggiormente coinvolti sono le vie urinarie, respiratorie, le ferite post-chirurgiche, il sangue (batteriemie), l'apparato gastro intestinale (diarree da Clostridium difficile); iii) lo $S$. aureus multiresistente (MRSA) risulta isolato nel 5\% di infezioni ospedaliere.

Come ogni anno il Centro europeo per la prevenzione e il controllo delle malattie (ECDC) pubblica l'Annual Epidemiological Report (AER) basato sui rapporti di sorveglianza (Disease surveillance reports) delle singole patologie e dei gruppi di malattie infettive.

Nel gennaio 2019, sono stati pubblicati i dati circa le ICA in Italia dovute ad Enterobatteri resistenti ai carbapenemi tra il 2014 e $2017 .{ }^{8-10}$ I dati raccolti dal sistema di sorveglianza nazionale per le batteriemie da enterobatteri produttori di carbapenemasi (CPE), istituito dal ministero della Salute nel 2013, mostrano come in Italia il fenomeno della resistenza ai carbapenemi, soprattutto nella specie batterica $K$. pneumoniae, sia diventato una seria minaccia per la salute pubblica, tanto da rappresentare una buona parte dei circa 10 mila morti l'anno causati in Italia dall'antibiotico resistenza. I casi segnalati di batteriemie da $K$. pneumoniae ed $E$. coli hanno mostrato un incremento nel tempo passando da circa 1400 casi nel 2014 a più di 2000 casi nel 2017, con un tasso di incidenza che nel 2017 ha raggiunto 3,6 casi su 100 mila residenti. I casi di batteriemie da CPE sono diffusi in tutto il territorio italiano (con evidenti differenze regionali nei tassi di incidenza) e rappresentano un esempio di in- fezione correlata all'assistenza sanitaria (ICA) coinvolgendo principalmente soggetti maschi con più di 60 anni di età $(71 \%)$, ospedalizzati $(87,2 \%)$ e ricoverati nei reparti ad alta intensità di cura spesso come conseguenza di procedure mediche invasive.

È necessario quindi continuare a rafforzare le misure di controllo per le ICA in tutto il territorio nazionale, al fine di ridurre il numero di infezioni correlate all'assistenza da Enterobatteri resistenti ai Carbapenemi. ${ }^{11-14}$

\section{Le strategie operative}

Appare quindi necessario, per contenere il fenomeno a macchia d'olio delle ICA, adottare precauzioni standard e definire la istituzione di sistemi di sorveglianza attiva tramite: i) monitoraggio dell'incidenza dei diversi tipi di infezione (complicanze infettive secondarie ad interventi chirurgici, infezioni in area critica); ii) attivazione di un protocollo di sorveglianza attiva, tramite la rete dei laboratori di microbiologia, per identificare gli eventi epidemici e definire gli agenti patogeni prevalenti con i loro profili di resistenza; iii) organizzazione di indagini sulla diffusione delle infezioni.

È bene tener presente che un inopportuno utilizzo degli antibiotici si ripercuoterà negativamente sulla diffusione delle ICA, per cui le misure di strategie di prevenzione e controllo sono quindi fondamentali.

La commissione tecnica responsabile della lotta contro le infezioni ospedaliere (CIO: Comitato per le Infezioni Ospedaliere) deve attuare le seguenti strategie: i) organizzare un sistema di sorveglianza epidemiologica delle infezioni ospedaliere; ii) codificare e organizzare le misure di prevenzione; iii) provvedere ad una adeguata informazione al personale sui programmi di sorveglianza e controllo (formazione continua del personale); iv) adottare una corretta politica di gestione della terapia antibiotica; v) creare proposte organizzative in merito a situazioni di particolare rischio infettivo; vi) effettuare REPORT annuali sulla situazione epidemiologica locale; vii) effettuare AUDIT sull'adesione ai protocolli-procedure e manuali; viii) effettuare proposte in ordine a prevenzione, formazione, ricerca.

Le precauzioni standard si applicano a tutti i pazienti, indipendentemente dalla sospetta o confermata presenza di un agente infettivo ed hanno lo scopo di ridurre il rischio di trasmissione di patogeni in ambito ospedaliero da pazienti portatori di infezioni diagnosticate o non (ancora) diagnosticate.

Esse sono rappresentate da: i) lavaggio delle mani; ii) uso di dpi (dispositivi protezione individuale); iii) smaltimento corretto taglienti e acuminati; iv) utilizzo della camera singola; v) igiene respiratoria; vi) collocazione del paziente; vii) presidi per l'assistenza e attrezzature sanitarie. 
Il Piano Nazionale della Prevenzione (2014-2018) ha stimato che il consumo di prodotti idroalcolici per l'igiene delle mani è inferiore a 10 litri per 1000 giornate di degenza (la categoria più bassa in Europa), contro una media europea di 18,7 litri/1000. Il Gold Standard è stimato essere intorno ai 20 litri/1000, quindi l'Italia è risultata essere nettamente inferiore alla media. ${ }^{15,16}$

In Italia, come già sottolineato, molto frequenti sono le ICA da CPE (Enterobatteri produttori di Carbapenemasi). Il controllo di tali infezioni passa attraverso delle norme ben precise: i) utilizzo scrupoloso delle precauzioni da contatto, ovvero igiene delle mani prima e dopo il contatto con il paziente colonizzato o infetto da cpe, uso di guanti e sovra-camice, intensificazione dell'igiene ambientale; ii) isolamento dei pazienti colonizzati/infetti in stanza singola con bagno dedicato o loro raggruppamento in aree dedicate dell'ospedale (cohorting); iii) ove possibile, assistenza dei pazienti colonizzati/infetti da CPE da parte di personale sanitario dedicato; iv) educazione/formazione del personale sanitario sulle misure di sorveglianza e controllo contro le infezioni da CPE.

Per la gestione di pazienti colonizzati/infetti da CPE è importante: i) la presenza di esperti nel controllo delle infezioni (gruppo operativo CIO, infettivologo, microbiologo, infermieri addetti al controllo); ii) la messa in atto di protocolli/procedure contestualizzate; iii) una programmazione per la corretta gestione degli antibiotici; iv) un laboratorio di microbiologia attivo per la diagnosi e l'immediata segnalazione a Direzione Sanitaria e al Team dei professionisti dedicati al controllo delle infezioni; v) l'attuazione di programmi di sorveglianza; vi) l'adozione di precauzioni da contatto (isolamento o cohorting).

La Task Force aziendale (secondo i criteri della C.M. 52/1985) prevede per il paziente infetto: i) un infermiere dedicato; ii) un medico della Direzione Medica di Presidio Ospedaliero; iii) uno specialista in malattie infettive; iv) uno specialista in microbiologia; v) un farmacologo clinico; vi) un ingegnere clinico; vii) un tecnico amministrativo.

Il protocollo prevede una precisa strategia di sorveglianza e controllo, ovvero screening, follow-up, sorveglianza attiva contatti.

È importante, inoltre, chiarire le modalità di trasferimento e dimissione, ovvero la comunicazione dello stato di portatore (cartella clinica/lettera di dimissione, contatto telefonico diretto, personale del servizio di trasporto, struttura/reparto accettante). Infatti, in caso di dimissione a domicilio, è bene fornire un opuscolo informativo al paziente e ai suoi familiari anche come sistema di alert in caso di re-ricovero nella stessa Struttura.

La diagnosi di laboratorio è fondamentale nello screening dei pazienti colonizzati da CPE ed il tempo di refertazione non deve superare le 48 ore (il timing delle tecniche di biologia molecolare è pari a circa 2 ore).

È bene attuare le adeguate misure di contenimento della trasmissione, mediante semplici regole, come già descritto. Dopo la diagnosi di laboratorio, è bene iniziare la terapia della infezione da CPE.

Infine, è importante ricordare che per tali infezioni, esiste un monitoraggio regionale delle attività di sorveglianza (Reporting e Indicatori), ovvero i dati devono trasmessi entro il $30^{\circ}$ giorno del mese successivo da un responsabile indicato.

La sorveglianza attiva dei pazienti colonizzati/infetti da CPE si effettua mediante screening specifico con tampone rettale o coprocoltura per: i) tutti i contatti di pazienti colonizzati/infetti da CPE, ovvero ciascun paziente assistito dalla stessa equipe di paziente colonizzato/infetto da CPE; ii) tutti i pazienti precedentemente identificati come colonizzati/infetti, nuovamente ricoverati in ospedale; iii) i pazienti ricoverati o trasferiti da reparti a rischio (UTI, Oncologia, Ematologia, Neuro Riabilitazione/Unità Spinale e Chirurgia dei trapianti); iv) pazienti provenienti da altro ospedale, o ricoverati in un altro ospedale negli ultimi 3 mesi o provenienti da strutture territoriali per anziani; v) tutti i pazienti provenienti da Paesi endemici per infezioni da CPE (U.S.A., Grecia, Cipro, India, Pakistan, Colombia, Israele, Porto Rico).

Dovranno essere isolati i pazienti ad alto rischio di colonizzazione ed i pazienti con un risultato preliminare di laboratorio positivo, in attesa della conferma fenotipica.

La sorveglianza passiva rappresenta la modalità di raccolta e flusso di dati per il sistema di sorveglianza nazionale per CPE. Il Referente di Laboratorio preposto della struttura ospedaliera compila la scheda del caso e la trasmette entro 48 ore sia alla Direzione Sanitaria dell'Azienda/Presidio Ospedaliero, sia al Dipartimento di Prevenzione ASL (pazienti strutture residenziali/domiciliari), che indaga circa il luogo di insorgenza dei sintomi, l'origine della batteriemia e l'esito dell'infezione, trasmettendo i dati entro 7 giorni alla Regione.

Gli alert microbiologici sono le segnalazioni che il Laboratorio di Microbiologia deve fare ai reparti qualora vengano riscontrati casi di singoli pazienti con infezione sostenuta da alcuni microrganismi definiti Germi sentinella. La segnalazione del laboratorio alla Direzione Sanitaria comporta l'attivazione di una scheda di rilevazione e consente di dare puntuali indicazioni assistenziali al personale di reparto, con applicazione delle misure di isolamento e contenimento del fenomeno.

La prevenzione del contagio passa per l'igiene degli ambienti (igiene del letto, sanitizzazione, microclima), l'igiene dei dispositivi (pulizia, disinfezione, sterilizzazione), l'igiene delle mani. 
L'attivazione delle misure di isolamento non è legata solo all'infezione ma anche alla semplice colonizzazione del paziente rilevata dalla positività microbiologica del materiale inviato in Laboratorio di Microbiologia.

L'organizzazione prevede la ottimizzazione delle misure di strategia e prevenzione delle ICA ed il Risk Management non può prescindere dall'aggiornamento continuo di Linee Guida e Protocolli per il trattamento di queste patologie, mediante una verifica continua di aderenza a documenti. Emerge, quindi, la necessità di risorse adeguate. ${ }^{17,18}$

Elementi fondamentali per evitare l'utilizzo incongruo dei farmaci sono rappresentati dalla ricognizione e dalla riconciliazione farmacologica.

La ricognizione consiste nella raccolta di informazioni, complete e accurate sul paziente e sui medicinali che assume, che sono indispensabili per una prescrizione corretta. La riconciliazione va effettuata non appena disponibili i dati della ricognizione e prima della prescrizione; in alcuni casi, dettati dall'urgenza e contemplati dalla procedura, può essere espletata successivamente. Il medico, prima di eseguire la prescrizione farmacologica, prende visione degli esiti della ricognizione ed effettua la riconciliazione, confrontando la terapia in corso con le disposizioni (prescrizioni ex novo, modifiche) ritenute necessarie per l'attuale circostanza clinica.

Non tutte le infezioni correlate all'assistenza sono prevenibili: è, quindi, opportuno sorvegliare quelle che sono attribuibili a problemi nella qualità dell'assistenza. In genere, si possono prevenire le infezioni associate a determinate procedure, attraverso una riduzione delle procedure non necessarie, la scelta di presidi più sicuri, l'adozione di misure di assistenza al paziente che garantiscano condizioni asettiche. Per arginare le ICA è necessario mettere in atto procedure di Risk Management che coinvolgano team di specialisti in formazione continua.

\section{Bibliografia}

1. Sherwin B. Nuland, Il morbo dei dottori. La strana storia di Ignác Semmelweis, Edizioni Codice, 2004.

2. Parascandola J (November-December 1996). "From MCWA to CDC - origins of the Centers for Disease Control and Prevention". Public Health Reports. 111 (6): 549-51.

3. Sledge, Daniel (2012). "War, Tropical Disease, and the Emergence of National Public Health Capacity in the
United States". Studies in American Political Development.

4. ISS Bollettino Epidemiologico Nazionale aprile 1985.

5. Haley R.W. et al. The efficacy of infection surveillance and control programs in preventing nosocomial infections in US hospitals. Am J Epidemiol. 1985 Feb;121(2):182-205.

6. Circolare Ministero della Sanità n. 52/1985 "Lotta contro le infezioni ospedaliere".

7. "Fondi integrativi sanitari finalizzati a fornire prestazioni aggiuntive rispetto a quelle assicurate dal Servizio sanitario nazionale" Decreto legislativo, N. 517/93, 7 dicembre 1993.

8. Iacchini $\mathrm{S}$ et al. Bloodstream infections due to carbapenemase-producing Enterobacteriaceae in Italy: results from nationwide surveillance, 2014 to 2017", Eurosurveillance Volume 24, Issue 5, 31/jan 2019.

9. Fontana et al: Emergence of KPC-producing Klebsiella pneumoniae in Italy, BMC Research Notes 2010, 3:40 http://www.biomedcentral.com/1756-0500/3/40.

10. Silvia Munoz-Price, et al. Clinical epidemiology of the global expansion of Klebsiella pneumoniae carbapenemases The Lancet Review| Volume 13, ISSUE 9, P785796, September 01, 2013.

11. DJ Weber, WA Rutala Lessons from outbreaks associated with bronchoscopy - Infection Control \& Hospital Epidemiology, 2001 - cambridge.org.

12. Merle A et al. Knowledge and opinion of surgical patients regarding nosocomial infections, Journal of Hospital Infection 2005, 60: 169-171.

13. Ongoing Multistate Outbreak of Escherichia coli serotype O157:H7 Infections Associated with Consumption of Fresh Spinach — United States, September 2006 MMWR Dispatch Vol. 55 / September 26, 2006.

14. "Sorveglianza, e controllo delle infezioni da batteri produttori di Carbapenemasi (CPE)" Circolare Ministeriale. Febbraio 2013.

15. Manuale di formazione per il Governo Clinico: la sicurezza dei pazienti e degli operatori: Ministero della Salute, gennaio 2012 .

16. Tura G., Moro M.L. Epidemie di infezioni correlate all'assistenza sanitaria, Sorveglianza e controllo, Collana Dossier dell'Agenzia sanitaria regionale dell'Emilia-Romagna, Bologna, 2006.

17. Centers for Disease Control and Prevention. Guideline for Hand Hygiene in Health Care Settings: Recommendations of the Healthcare Infection Control Practices Advisory Committee and the HICPAC/SHEA/APIC/IDSA Hand Hygiene Task Force. MMWR 2002;51.

18. Schwaber M.J. et al: the Israel Carbapenem-Resistant Enterobacteriaceae Working Group. Containment of a Country-wide Outbreak of Carbapenem-Resistant Klebsiella Pneumoniae in Israeli Hospitals via a Nationally Implemented Intervention. Clin Infect Dis. $2011 \mathrm{Apr}$ $1 ; 52(7): 848-55$. 


\title{
Safe Handover
}

\author{
Roberta Rapetti \\ Coordinatore Infermieristico S.C. Coordinamento Professioni Sanitarie Territoriale, Referente Ricerca, Buone Pratiche, \\ Innovazione, ASL 2 Sistema Sanitario Regione Liguria, Italia
}

\section{Introduzione}

Handover è definito come il processo di trasferimento della responsabilità professionale e della presa in carico per alcuni o tutti gli aspetti della cura di un paziente o un gruppo di pazienti, ad un'altra persona o una equipe professionale, su base temporanea o permanente. ${ }^{1}$

Il passaggio delle informazioni cliniche è stato identificato, sia a livello nazionale che internazionale, una fase critica all'interno del percorso di cura. ${ }^{2}$

In ambito sanitario, Handover, è una delle procedure a maggior rischio e, se svolta in modo improprio, può determinare errori e danni ai pazienti stessi. ${ }^{2}$

Un sondaggio ha riconosciuto che la scarsa comunicazione, le differenti culture e percezioni, le credenze professionali, il disagio e la complessità della malattia come sono fattori contribuenti alla variabilità del processo comunicativo. ${ }^{3}$

Nelle situazioni lavorative, risulta fondamentale avvalersi di una modalità comunicativa univoca al fine di per ridurre gli errori e limitare la perdita di informazioni. $^{3}$

Handover inadeguati possono condurre a dispersione delle risorse con effetti rilevanti sulla sicurezza degli utenti, creando ritardi nella diagnosi e nel trattamento, ripetizioni o posticipazioni di esami e possibili eventi farmacologici avversi. ${ }^{4}$

Dai numerosi eventi sfavorevoli censiti, è emersa l'esigenza di implementare registri comunicativi ef-

Corrispondente: Roberta Rapetti, Coordinatore Infermieristico, S.C. Coordinamento Professioni Sanitarie Territoriale, Referente Ricerca, Buone Pratiche, Innovazione, ASL 2 Sistema Sanitario Regione Liguria, Via Genova 14, Savona, Italia.

E-mail: rapettirobi@gmail.com

Articolo pubblicato secondo la Creative Commons Attribution NonCommercial 4.0 License (CC BY-NC 4.0).

${ }^{\circ}$ Copyright: the Author(s), 2019

Licensee PAGEPress, Italy

QUADERNI - Italian Journal of Medicine 2019; 7(6):91-94 ficaci al fine di sostenere le transizioni di cura e il passaggio tempestivo, accurato e appropriato di informazioni. ${ }^{3}$

\section{Key messages}

Handover, processo di trasferimento della responsabilità professionale per alcuni o tutti gli aspetti della cura, su base temporanea o permanente, costituisce una norma di buona pratica clinica.

Il completo e corretto trasferimento delle informazioni risulta prioritario nel garantire la continuità e la sicurezza assistenziale.

\section{Comunicazione e handover}

La comunicazione è la variabile più importante di un buon lavoro di gruppo ed è fondamentale per mantenere la sicurezza dell'ambiente lavorativo degli operatori e del paziente. ${ }^{5}$ Lo scambio di informazioni, feedback, risposte, idee e opinioni consente la diffusione delle conoscenze, l'istituzione di relazioni, definisce schemi di comportamento prevedibili, permette di focalizzare l'attenzione sulla funzione e costituisce un efficace strumento di gestione. ${ }^{6}$

La comunicazione comprende una varietà di elementi rilevanti quali l'invio di messaggi in modo chiaro e conciso, l'inclusione delle variabili di contesto, la ricezione del riscontro, in particolare attraverso l'ascolto e il riconoscimento di eventuali barriere ed un suo efficace e pieno espletamento. ${ }^{7}$

Una relazione inefficace risulta essere causa frequente numerosi incidenti in diversi settori, sia in ambito sanitario che non. ${ }^{7}$

La comunicazione è, infatti una abilità che può essere appresa, sviluppata, migliorata e la formazione risulta un elemento indispensabile per uniformare $\mathrm{i}$ comportamenti. $^{8}$

\section{Key messages}

La comunicazione è il sistema attraverso il quale le persone interagiscono e il loro sapere organizzativo prende forma e si diffonde. Un registro comunicativo impostato e condiviso consente di evitare ritardi nella 
diagnosi e nel trattamento, ripetizione o posticipazione di esami ed eventi avversi soprattutto farmacologici.

\section{Handover: aspetti strutturali}

Negli ultimi anni, in ambito sanitario, è stata posta grande attenzione al processo di Handover come principio vitale di tutela della sicurezza e come strumento di miglioramento della qualità assistenziale. ${ }^{3}$ Nonostante ciò, a tutt'oggi, permangono ostacoli ad un'adeguata circolazione delle informazioni. ${ }^{3}$

Gli attuali modelli organizzativi evidenziano la necessità di un cambio di paradigma nella presa in carico della persona e, sottolineano che tutti gli attori coinvolti, ad ogni livello, devono riconoscere il processo di handover, come tempo dedicato alla cura.

Frequentemente, le informazioni trasferite risultano essere eterogenee nei contenuti e talvolta anche irrilevanti per il percorso assistenziale. ${ }^{9}$

È pertanto, indispensabile per gli operatori sanitari essere formati e addestrati ad utilizzare strumenti utili a codificare il processo. ${ }^{9}$

Handover va analizzato in relazione a tre aspetti principali: contenuto, modello e luogo, cosa, come e dove comunicare ${ }^{1}$ (Tabella 1).

Il contenuto è caratterizzato dalle informazioni riferite ad ogni degente, che devono essere accurate, chiare, brevi e tempestive; il passaggio può essere facilitato dall'uso di sistemi di registrazione cartacei o elettronici. ${ }^{10}$

Il modello include il registro comunicativo, l'uso di eventuali checklist di sicurezza e/o liste mnemoniche o una loro combinazione. ${ }^{10,11}$

Handover può svolgersi in più contesti, dal letto del malato a luoghi dedicati. ${ }^{12}$

Uno scambio idoneo di conoscenze risulta fondamentale per garantire il raggiungimento di una attenta consapevolezza situazionale, passata, presente e futura, e un modello mentale il più possibile corrispondente alla realtà.

\section{Key messages}

Il processo di handover circoscrive tre importanti aspetti: contenuto da trasmettere, modalità operativa e contesto organizzativo.

Il contenuto comprende le informazioni riferite ad ogni degente, che devono essere complete, chiare, brevi e tempestive.

La modalità operativa, include il registro comunicativo, l'uso di eventuali checklist di sicurezza e/o liste mnemoniche o la loro combinazione.

Il contesto, varia a seconda dell'organizzazione, e può essere bedside o altri luoghi dedicati.

\section{Handover: metodi e strumenti EBP}

La letteratura suggerisce molteplici strumenti per facilitare e standardizzare lo scambio interprofessionale di informazioni, tra questi l'approccio delle $5 P,{ }^{13}$ I Pass The Baton, ${ }^{14}$ SBAR,,${ }^{15}$ PVITAL $^{16}$ e ISBAR $3 .{ }^{3}$

Il metodo delle $5 P$ esprime le componenti della consegna tradizionale, identificando nella prima $\mathrm{P}$ la persona (nome, anni e genere), nella seconda l'organizzazione del percorso (diagnosi, trattamento, piano assistenziale e le successive fasi), nella terza il razionale della cura, nella quarta le potenziali problematiche e nella quinta le accortezze da mettere in atto per ogni degente. ${ }^{13}$

Il metodo I Pass The Baton fornisce una sequenza di atti da intraprendere durante i passaggi di cura al fine di migliorare la comunicazione e la presa in carico; inquadra l'attenzione su diversi aspetti tra cui: il paziente (I), i dati socio anagrafici (P), i principali disturbi, i parametri vitali, la diagnosi e la condizione attuale (A), i cambiamenti e la risposta ai trattamenti (S), le preoccupazioni per la sicurezza (S - score di rischio e allergie), le comorbidità, la storia familiare e la terapia in atto (B), le azioni intraprese (A), i tempi (T), le responsabilità operative $(\mathrm{O})$ e il piano da realizzare $(\mathrm{N}) \cdot{ }^{14}$

PVITAL è un metodo utilizzato dagli infermieri al letto del malato e si sviluppa mediante i seguenti con-

Tabella 1. Raccomandazioni operative (Safe handover: safe patients' guideline AMA, 2006).

\begin{tabular}{ll}
\hline Handover - Raccomandazioni operative & \\
\hline Chi dovrebbe essere coinvolto? & Tutti i professionisti interessati nel percorso di cura, in particolare il medico e l'infermiere \\
\hline Quando dovrebbe essere svolto? & $\begin{array}{l}\text { Ad orari prestabiliti (cambi turni, briefing e debriefing) per un massimo } 30 \text { minuti } \\
\text { Durante l'Handover, nessuna altra attività, ad esclusione delle situazioni urgenti, dovrebbe } \\
\text { essere intrapresa } \\
\text { Prevedere un sistema di presenza che garantisca la sicurezza delle cure }\end{array}$ \\
\hline Dove dovrebbe essere svolto? & Luogo dedicato dove sia possibile consultare la cartella clinica e la documentazione sanitaria \\
\hline Come dovrebbe essere organizzato? & \begin{tabular}{l} 
Guidato dalla figura più esperta mediante un registro comunicativo organizzato \\
\hline Che cosa è necessario che sia trasmesso?
\end{tabular} \\
$\begin{array}{l}\text { Informazioni rilevanti sui pazienti: } \\
\text { da rivalutare }\end{array}$ \\
$\begin{array}{l}\text { critici } \\
\text { da supervisionare nel turno successivo }\end{array}$
\end{tabular}


tenuti: presentazione del paziente con nome, età e problemi attuali (P), valutazione dei segni vitali (V), verifica del bilancio idro-elettrolitico (I), attuazione del piano assistenziale $(\mathrm{T})$, programmazione della dimissione (A) e verifica della documentazione sanitaria con la relativa completezza della cartella clinica $(\mathrm{L})^{16}$.

$S B A R$ è una tecnica utile per uniformare lo scambio di notizie, si presta alla comunicazione faccia a faccia, telefonica e/o scritta; infatti sono disponibili moltissimi moduli, nelle forme più diverse. Gli elementi caratterizzanti questa metodologia sono i seguenti: situazione con individuazione del problema (S), definizione del contesto (B), valutazione di parametri vitali, segni e sintomi (A), interventi da realizzare $(\mathrm{R}) .{ }^{15}$

La tecnica SBAR viene raccomandata per quesiti e riscontri rapidi, meno adatta per il passaggio delle consegne, in quanto potrebbe non raccogliere tutte le informazioni necessarie nel percorso clinico. ${ }^{15}$

Il metodo $I S B A R_{3}$ è composto da alcuni punti chiave, tra i quali la corretta identificazione degli operatori sanitari e del paziente (I), la descrizione della situazione socio-clinico-assistenziale (S), la valutazione della condizione esistente (B), l'esplicazione delle raccomandazioni cliniche, la rilettura del messaggio e la pausa di sicurezza per governare il rischio di errore $\left(\mathrm{R}_{3}\right)^{3}$ (Tabella 2).

Safety pause è un'iniziativa di qualità, della durata di pochi minuti, che aiuta a riconoscere i rischi potenziali e/o reali del contesto organizzativo al fine di salvaguardare la sicurezza, avvalendosi dell'approccio delle 4 P: patients, professionals processes, patterns.

$\mathrm{ISBAR}_{3}$ può essere utilizzato in molteplici circostanze, in particolare per lo scambio di consegne tra un turno e l'altro, nei briefing/debriefing, nei passaggi di cura, all'interno del dipartimento pediatrico e di emergenza, tra ospedale e territorio e come strumento di comunicazione professionale tra operatori e paziente/caregiver. $^{3}$
Le evidenze hanno definito $\mathrm{ISBAR}_{3}$ il registro di comunicazione interprofessionale più completo sia per qualità, sia per chiarezza del messaggio, fruibile in molti contesti assistenziali, dimostrando che l'aderenza a questo protocollo comunicativo riduce le omissioni di informazioni. ${ }^{3}$

Gli standard Joint Commission e l'Organizzazione Mondiale della Salute raccomandano 1'uso della metodica del read back al fine di garantire la sicurezza nelle cure. La procedura prevede che la persona ricevente ripeta a quella emittente la comunicazione acquisita o una specifica parte di essa, al solo scopo di ottenere conferma della corretta ricezione. ${ }^{3}$

Handover, è riconosciuto una buona pratica clinica, e come tale dovrebbe essere condotto mediante criteri di qualità e un registro comunicativo strutturato, ove possibile, face to face, supportato da documentazione sanitaria pertinente, accurata e aggiornata. ${ }^{3}$

\section{Key messages}

La letteratura suggerisce numerosi strumenti per il passaggio strutturato delle informazioni, tra i quali: 5 P, I Pass The Baton, PVITAL, SBAR e ISBAR .

WHO raccomanda l'utilizzo della metodica read back al fine di ottenere conferma della corretta ricezione del messaggio e garantire la massima sicurezza delle cure.

\section{Conclusioni}

È possibile affermare che, un sistema di handover codificato, l'uso di checklist condivise, una maggiore attenzione alla prevenzione e gestione del rischio clinico, una chiara individuazione di funzioni e responsabilità, un rafforzamento del team di lavoro e ove è possibile, la partecipazione della persona ricoverata, siano elementi cardine dell'organizzazione attuale e futura.

Tabella 2. ISBAR3 - National Communication (Clinical Handover) Guideline Development of patient care in acute and children's hospital services National Guideline No. 11. ISSN 2009-6259. November 2015.

\begin{tabular}{ll}
\hline $\mathbf{I}$ & Team curante e paziente \\
\hline $\mathbf{S}$ & $\begin{array}{l}\text { Valutazione della persona } \\
\text { Situazione }\end{array}$ \\
\hline $\mathbf{B}$ & Analisi della situazione clinica e delle criticità \\
Background & Riassunto conciso dei motivi del ricovero \\
\hline $\mathbf{A}$ & Terapia in atto \\
Assessment & Valutazione della situazione clinico-assistenziale attuale e dei segni vitali \\
\hline $\mathbf{R 3}$ & \\
Raccomandazioni & $\begin{array}{l}\text { Azioni da attuare } \\
\text { Read-back }\end{array}$ \\
Rischio & Conferma delle informazioni ricevute \\
\hline
\end{tabular}




\section{Bibliografia}

1. Safe handover: safe patients' guideline AMA, 2006.

2. McEwan JR, Atkinson S, Cadigan P, Ingham J. Handover - the need for best practice. Report to RCP Council, 2010.

3. National Communication (Clinical Handover) Guideline Development of patient care in acute and children's hospital services National Guideline No. 11. ISSN 20096259 November 2015.

4. The ossie guide to clinical handover improvement Australian commission on safety and quality in health care 2009.

5. Scott I.Tannenbaum, Rebecca L.Beard, EduardoSalas Chapter 5 Team Building and its Influence on Team Effectiveness: an Examination of Conceptual and Empirical Developments, Advances in Psychology 1992 Volume 82 Pages 117-153.

6. Chambers S Use of non-verbal communication skills to improve nursing care Br J Nurs. 2003 Jul 24-Aug 13;12 (14):874-8.

7. Rebecca Randell, Stephanie Wilson, Peter Woodward The Importance Of The Verbal Shift Handover Report:A Multi-Site Case Study International Journal Medical In-

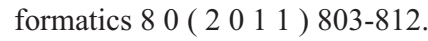

8. Wong MC, Yee KC, Turner P. A Structured Evidencebased Literature Review regarding the Effectiveness of Improvement Interventions in Clinical Handover, Australian Commission on Safety and Quality in Health Care (ACSQHC) 2008.
9. Ilan R, LeBaron CD, Christianson MK, et al Handover patterns: an observational study of critical care physicians, BMC Health Serv Res. 2012 Jan 10;12:11.

10. Chapter 32 Structured patient handovers Emergency and acute medical care in over 16s: service delivery and organisation NICE guideline National Institute for Health and Care Excellence July 2017.

11. Merten H, van Galen LS, Wagner C. Safe handover, BMJ. 2017 Oct 9;359:j4328.

12. Sean Gregory, Debra Tan, Michael Tilrico et al, Bedside Shift Reports What Does the Evidence Say? JONA Volume 44, Number 10, pp 541-545.

13. H\&HN research and Sentara Health Care, 2008.

14. Agency for Healthcare Research and Quality. Team STEPPSTM: national implementation July 28, 2011.

15. Clark E, Squire S, Heyme A, et al: The PACT Project: improving communication at handover. Med J Aust 2009; 190: 125-127.

16. NSW Health: Implementation toolkit - Standard key principles for clinical handover, September 2009 - Shannon Bakon, Lisa Wirihana, Martin Christensen et al, Nursing handovers: An integrative review of the different models and processes available Int J Nurs Pract 2017; e12520.

17. Thompson JE, Collett LW, Langbart MJ et al, Using the ISBAR handover tool in junior medical officer handover: a study in an Australian tertiary hospital Postgrad Med J. 2011 May;87(1027):340-4. 


\title{
Prevenzione dei rischi in Medicina Interna, casi particolari: atti autolesivi, vagabondaggio, intrappolamento nel paziente ospedalizzato
}

\author{
Roberto Nardi, ${ }^{1}$ Daniela Tirotta, ${ }^{2}$ Giuliano Pinna, ${ }^{3}$ Giampiero Pirini ${ }^{4}$ \\ ${ }^{1}$ Medicina Interna, Bologna; ${ }^{2}$ Medicina Interna, Cattolica (Rimini); ${ }^{3}$ Medicina Interna, Asti, ${ }^{4}$ Direttore Servizio Comune \\ Ingegneria Clinica, Azienda Ospedaliera-Universitaria di Ferrara, Italia
}

\section{Introduzione}

Nel 2013 il Ministero della Salute ha pubblicato un rapporto sugli eventi sentinella (eventi avversi, particolarmente gravi, potenzialmente evitabili, che possono comportare morte o grave danno al paziente). Nel periodo considerato (settembre 2005 - dicembre 2011) sono pervenute 1723 segnalazioni, di cui 1442 valide ai fini della produzione della reportistica. La morte o grave danno per caduta di paziente, con 321 segnalazioni, rappresenta l'evento più frequente, con una percentuale di casi pari al 22,3\% rispetto al totale degli eventi sentinella. La seconda categoria per numerosità (226 eventi, corrispondenti al 15,67\% del totale) è rappresentata dal Suicidio o tentato suicidio di paziente in ospedale. Tra i fattori contribuenti, la mancanza, inadeguatezza ed inosservanza di Linee-guida, raccomandazioni, protocolli assistenziali, procedure, insieme al problema di sotto-segnalazione di questi eventi sentinella. ${ }^{1}$

\section{Atti autolesivi e rischio di suicidio in ospedale}

Il suicidio in ospedale rappresenta un evento sentinella di particolare gravità, la cui prevenzione si basa su una appropriata valutazione delle condizioni del paziente. ${ }^{2}$ È opportuno che il personale medico e infermieristico, secondo le rispettive competenze professionali, presti l'attenzione ai fattori anamnestici, so-

Corrispondente: Roberto Nardi, Medicina Interna, Bologna, Italia.

E-mail: nardidoc48@gmail.com

Articolo pubblicato secondo la Creative Commons Attribution NonCommercial 4.0 License (CC BY-NC 4.0).

${ }^{\circ}$ Copyright: the Author(s), 2019

Licensee PAGEPress, Italy

QUADERNI - Italian Journal of Medicine 2019; 7(6):95-104 ciali, obiettivi e clinici, che possono configurare un aumentato rischio. ${ }^{3}$

\section{Quali possibili fattori di rischio e quali protettivi?}

Alcuni dei sintomi e/o i segni di sospetto e i possibili fattori di rischio di atti autolesivi in ospedale sono riportati nella Figura 1 e seguenti. ${ }^{4}$

Spesso il soggetto a rischio di suicidio manifesta espressioni quali: vorrei essere morto, mi sento solo, non riesco a fare nulla, non posso più andare avanti così, sono un perdente, gli altri staranno meglio senza di me. Il rischio di suicidio si associa a sentimenti di disperazione, di rabbia incontrollabile e senza via d'uscita. Il rischio può essere associato al consumo di alcol e droga, alla perdita di amicizie, della famiglia e dei contatti sociali. ${ }^{5}$ Le persone anziane hanno un rischio maggiore di suicidio, ma, nonostante ciò, tale fenomeno è molto sottovalutato nella sanità pubblica e nella ricerca medica. ${ }^{6}$

\section{Individuazione del paziente a rischio di possibili comportamenti autolesivi e azioni successive}

Nell'individuazione dei pazienti maggiormente propensi ad atti autolesivi si richiama il fatto che la singola domanda diretta: sta pensando al suicidio? ha una sensibilità del $83,3 \%$ ed una specificità del $97,7 \% .{ }^{7}$ Uno strumento impiegato per la valutazione del paziente tendenzialmente suicida è la scala MADRS (Montgomery-Asberg Depression Rating Scale $)^{8,9}$ che considera i seguenti items: $0=$ non presenta evidenti tematiche suicidarie; $1-2=$ il paziente è stanco della vita; sono presenti fugaci idee di suicidio; 3-4= sarebbe meglio essere morto; ricorrenti idee di suicidio; il suicidio è considerato come una soluzione possibile, mancano tuttavia progetti o intenzioni precise; 5-6= progetti espliciti di suicidio, se si presentasse l'occasione. Preparativi di suicidio. Alla luce di tali elementi, la richiesta di consulenza psichiatrica, meglio se urgente, dovrebbe contemplare, nei casi a rischio, alcuni quesiti espliciti, con adeguate modalità d'interazione tempestive e con un feed back bi-dire- 
zionale. Le refertazioni dello specialista psichiatra, sempre ben documentate nella cartella clinica, dovrebbero corrispondere in modo congruente alle esplicite richieste dell'internista, proprio per garantire insieme la giusta protezione del paziente (Tabella 1).

La Figura 2 riporta in maniera schematica le modalità di valutazione dei pazienti e le corrispettive azioni/controlli da implementare.

I punteggi della scala MADRS corrispondenti a quelli indicati determinano possibili alternative decisionali: $0=$ nessuna necessità di attenzioni specifiche; 1-2= necessità di un'assistenza attenta da parte del personale normalmente presente in reparto; $3-4=n e-$ cessità di un 'assistenza attenta e continuativa nelle 24 ore da parte del personale normalmente presente in reparto elo da parte dei familiari elo delegati; 5-6= necessità di un controllo diretto, meticoloso e continuativo nelle 24 ore da parte dello staff medico, infermieristico e ausiliario del reparto.

Alcune situazioni ambientali possono facilitare in ospedale il rischio di autolesionismo. In tale ambito è evidentemente necessario il coinvolgimento degli organi tecnico-direzionali dell'Ospedale, ai fini del dovuto controllo. Situazioni a rischio sono, ad esempio:

Tabella 1. Quesiti espliciti da formulare nella richiesta di consulenza psichiatrica urgente.
a) Il paziente è a rischio di atti autolesivi?
b) Quale è il livello di rischio?
c) Quale è lo stato di capacità d'intendere e volere del paziente?
d) La situazione contestuale consente una gestione sicura del paziente?
e) Quali provvedimenti devono essere intrapresi in base al rischio di atti autolesivi, alla capacità e alle condizioni di sicurezza?
f) Il paziente deve essere trasferito in ambiente specialistico psichiatrico?
g) Se non è disponibile il posto o non è possibile trasferire il paziente presso un centro specialistico psichiatrico quali provvedimenti devono essere adottati da parte della struttura che ha in cura il paziente?
h) Quali modalità di rivalutazione specialistica psichiatrica sono previste nel follow up a breve termine?
i) Entro quanto tempo il paziente deve essere rivalutato dallo specialista psichiatra insieme all'internista?

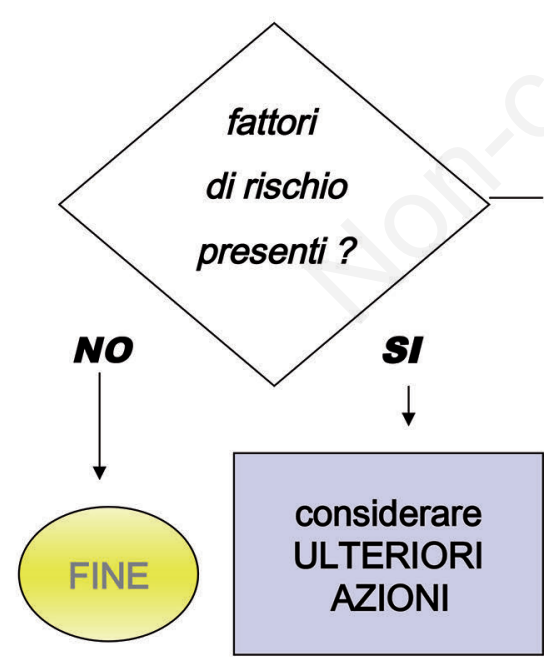

\begin{tabular}{|c|c|c|c|}
\hline & FATTORI DI RISCHIO & FATTORI PROTETTIVI & \\
\hline & Specifici stati emotivi, età avanzata & \multirow{2}{*}{ Buon supporto } & \\
\hline & Psicopatologia nota o dubbia (farmaci) & & \\
\hline & Umiliazione / rifiuto / licenziamento & Famiglia unita & \\
\hline & Precedenti tentati suicidi & \multirow{2}{*}{ Appartenenza a comunità } & \\
\hline & Recente lutto / ricorrenza luttuosa & & \\
\hline & Intossicazione alcolica o droghe d' abuso & Capacità di coping* & \\
\hline & Divorzio, vedovanza, famiglia assente & \multirow{2}{*}{ Sistema di valori } & \\
\hline & Patologia inguaribile/cronica & & \\
\hline & Difficoltà economiche, sfratto & \multirow{2}{*}{$\begin{array}{l}\text { Capacità di ricerca di assistenza } \\
\text { sanitaria }\end{array}$} & ii \\
\hline & Azioni legali pendenti & & \\
\hline & Conflitti religiosi /culturali / famigliari & \multirow{5}{*}{$\begin{array}{l}\text { - cancro o prognosi infausta } \\
\text { - malattia inguaribile } \\
\text { - dolore severo / insopportabile } \\
\text { - asma, malattie polmonari, dispnea } \\
\text { - HIV } \\
\text { - disabilità e dipendenza da altri } \\
\text { - epilessia } \\
\text { - alcoolismo, tossicodipendenza } \\
\text { - farmaci: } \\
\text {-beta-bloccanti, reserpina, } \\
\text {-clonidina, antipertensivi, } \\
\text { - neurolettici } \\
\text { - wuthdrawal syndrome } \\
\text { - altre ... }\end{array}$} & \\
\hline & Scarso supporto sociale / famigliare & & \\
\hline & Incapacità di accettare aiuto & & \\
\hline & Problemi connessi allo stato di straniero & & \\
\hline & ENTE & & \\
\hline
\end{tabular}

Figura 1. Fattori di rischio e fattori protettivi. *Capacità di coping o capacità di adattamento: indica la capacità da parte dell'individuo di adattarsi, in senso psico-dinamico a problemi personali, eventi stressanti, lutti e conflitti. 


\section{PAZIENTE A RISCHIO DI ATTI AUTOLESIVI IN OSPEDALE 1}

\section{Sono presenti segni/sintomi di sospetto}

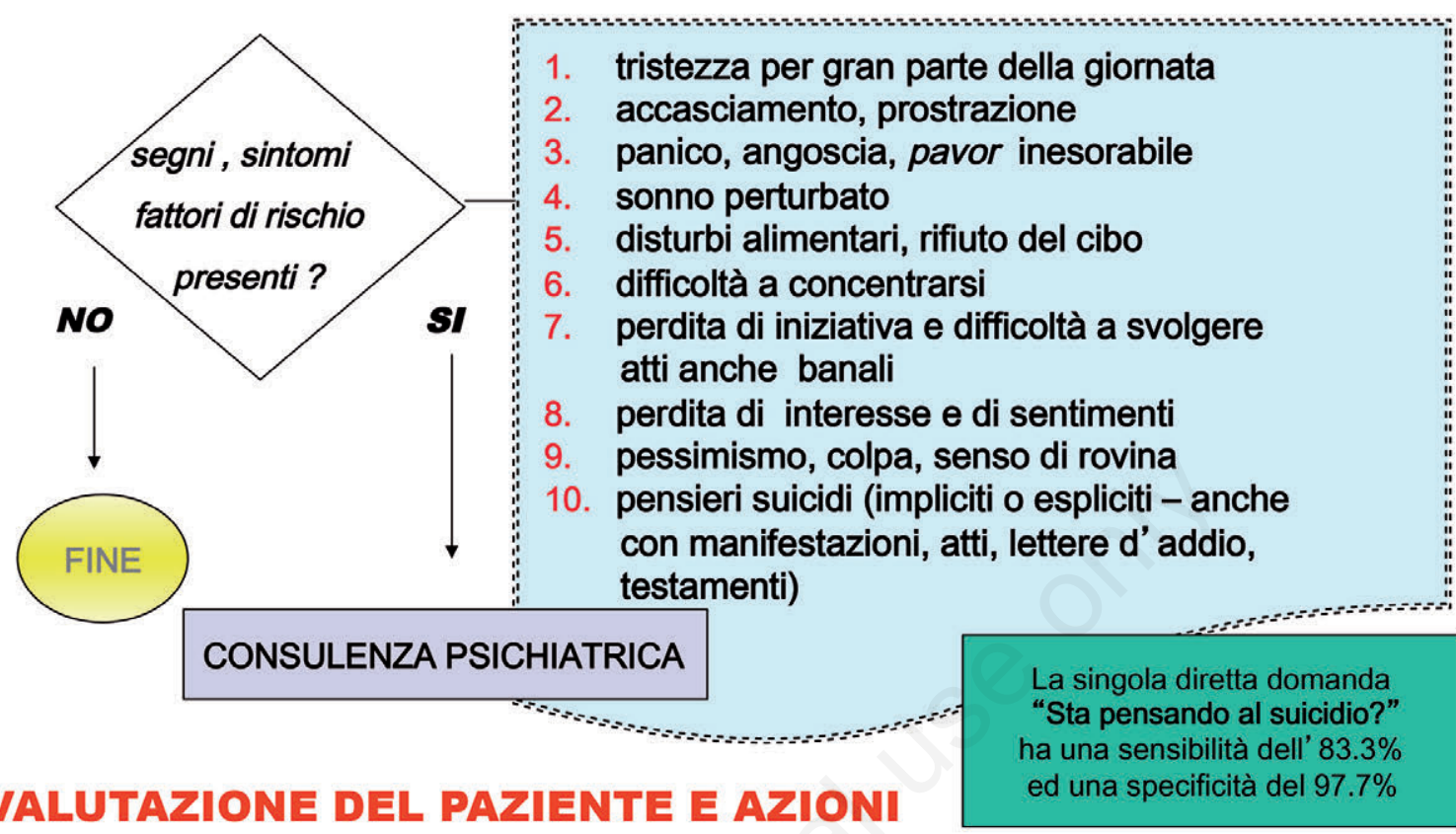

\section{PAZIENTE A RISCHIO DI ATTI AUTOLESIVI IN OSPEDALE 2}

Quali provvedimenti devono essere intrapresi in base al rischio di atti auto-lesivi, allo stato di capacità del paziente e al contesto assistenziale ?

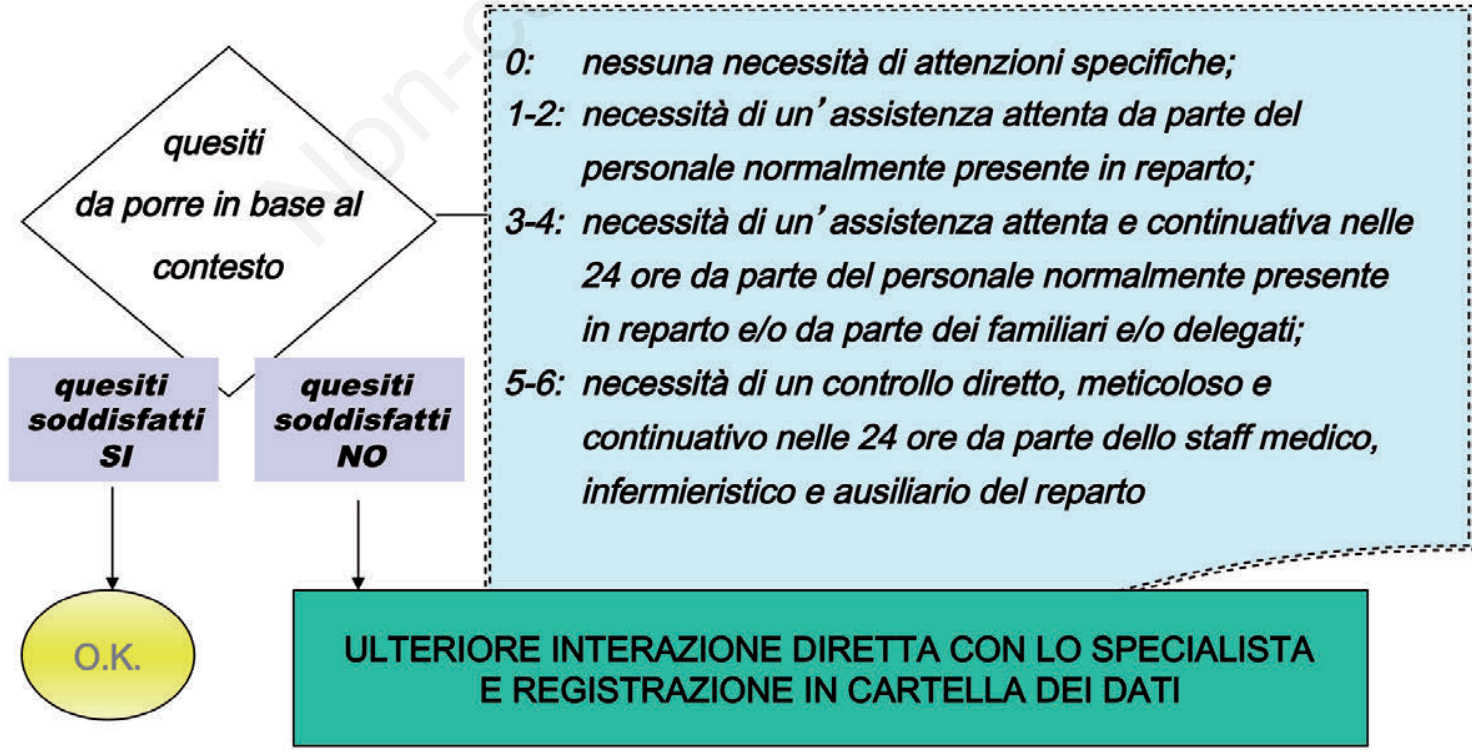

COMUNICAZIONE TRA PROFESSIONISTI:

MODALITA' DI REFERTAZIONE PSICHIATRICA ED INTERAZIONE RECIPROCA

Figura 2. A) Il paziente a rischio di atti autolesivi in ospedale; B) azioni da implementare in un reparto internistico. 
finestre non bloccate, cavi elettrici non sottotraccia, prese di corrente non protette, porte, balconi, scale ed accessi all'esterno del reparto non vigilati da sistemi di controllo, eccetera. Nel reparto di degenza, nei casi a rischio, dovrebbero essere eliminate tutte le possibili fonti di autolesionismo: cinture, cravatte, lacci, sacchetti di plastica, vetreria e tubi (set da flebo), aghi, termometri, accendini, fiammiferi, coltelli, forbici, lamette, rasoi, farmaci (conservati non assunti, di proprietà): digitale, warfarin, psicofarmaci, neurolettici, barbiturici, eccetera. Ulteriori specificazioni in merito sono schematicamente riportate in Tabella $2{ }^{3}$

Dal punto di vista medico-legale, come ribadito da recenti sentenze del Tribunale di Milano (Trib_Milano_sez_I_28_febbraio_2017_n_2503_gu_Boroni) riguardo alla responsabilità della struttura ospedaliera per il suicidio di un paziente ricoverato affetto da disturbi psichici, l'obbligo di vigilanza e protezione del paziente scaturisce fin dall'accettazione del paziente, a prescindere dalla capacità di intendere e di volere di questi, né esige che il paziente sia sottoposto ad un trattamento sanitario obbligatorio. Anche una persona perfettamente capace di intendere e di volere, infatti, può aver bisogno di vigilanza e protezione per evitare che si faccia del male (come nel caso di degente non autosufficiente). L'obbligo di protezione scaturente dal contratto (art. 1374 c.c.) non è teleologicamente orientato: non va adempiuto solo se si tratti di prevenire il rischio $A$ od il rischio $B$, ma va adempiuto omni modo, al fine di prevenire tutti i rischi potenzialmente incombenti sul degente, alla sola condizione che rientrino nello spettro della prevedi-

Tabella 2. Raccomandazioni del Gruppo di Lavoro della Regione Emilia Romagna per la prevenzione delle condotte suicidarie in Ospedale.

a. Impegno programmatico della Direzione: ogni Organizzazione dovrebbe esplicitare il proprio impegno programmatico a elaborare un piano di gestione del rischio suicidario in ambito ospedaliero, come parte integrante del Piano di programma Aziendale di gestione del rischio, sensibilizzando il personale a segnalare prontamente gli eventi sentinella del suicidio e tentato suicidio, effettuando il relativo monitoraggio; programmando cicli di formazione per il personale; stilando un report annuale sulle azioni ed i risultati raggiunti;

b. Valutazione del rischio suicidario intesa come processo, effettuata non solo all'ammissione, ma anche in frangenti clinici importanti durante il trattamento e alla dimissione;

c. Anamnesi e valutazione medico/infermieristica:

- Indagare soprattutto: pregressi atti autolesivi, eventi avversi recenti, come lutti o malattie, abusi sessuali, familiarità per suicidio, primo accesso in assoluto di paziente anziano in ospedale;

- esame obiettivo: attenzione ad eventuali segni di recenti/pregressi comportamenti autolesionistici e/o suicidari, segni di abuso da alcool o stupefacenti, eventuali segni di astinenza;

- diagnosi di specifiche condizioni cliniche connesse a maggior rischio suicidario:

- patologia psichiatrica grave (depressione, disturbo bipolare, schizofrenia ed altri disturbi psicotici, disordini della personalità con comportamento aggressivo e impulsivo);

- abuso/dipendenza da alcool, stupefacenti e/o psicofarmaci, gioco d'azzardo patologico;

- sindromi cerebrali organiche;

- altri possibili sintomi/disturbi comportamentali (disperazione, impulsività, esternazione di idee di suicidio, negazione incongrua/contraddittoria, ricerca di mezzi letali, ansia, attacchi di panico, agitazione, insonnia, cambiamenti di umore, rabbia, abulia, ritiro sociale);

- diagnosi multiple;

- patologia terminale.

d. Interazione con lo psichiatra ed instaurazione nel ricovero delle idonee misure, considerando che i primi giorni dopo l'ammissione (per solitudine, paura, esperienze depersonalizzanti stigmatizzate in ambiente sconosciuto) e dopo la dimissione (per incompleto miglioramento clinico, mancata instaurazione di un'alleanza terapeutica, brusca sospensione delle cure e mancanza di un affidamento terapeutico extraospedaliero, esposizione a stress esterno e scarso supporto familiare) sono i più critici;

e. Instaurazione di caratteristiche di sicurezza ambientale e strutturale, che riguardano tutti i pazienti, a prescindere dal rischio individuato, tra cui:

- Posate di plastica e piatti infrangibili;

- Quadri ben fissati al muro, con vetri di sicurezza; specchi di sicurezza nei bagni;

- Strumenti e attrezzature (stetoscopi, strumenti taglienti, farmaci) attentamente custoditi;

- Sistemi antintrusione anche nei locali riservati al personale;

- Vetri antisfondamento e/o ringhiere;

- Maniglie delle porte con requisiti di sicurezza;

- Protezione dei raccordi per ossigeno e aspirazione;

- Soffioni doccia fissi e con disegno che ne impedisce l'appiglio;

- Letti ad altezza variabile;

- Campanelli di allarme non appesi a soffitto/pareti;

- Pavimenti e rivestimenti parietali in linoleum per attutire gli effetti di eventuali cadute o urti in camere di degenza;

- Eventuali impianti di videosorveglianza in aree non presidiate o critiche.

f. Trattare il suicidio ed il tentato suicidio come evento sentinella 
bilità. La presunzione di cui all'art. 1218 c.c., gravante sulla clinica, è infatti una presunzione di colpa da cui il soggetto onerato si libera dimostrando di avere tenuto una condotta diligente. La diligenza esigibile dalla struttura ospedaliera consiste in un 'adeguata sorveglianza del degente, che sia o meno capace di intendere e di volere, che deve avvenire innanzitutto nel reparto di degenza: è li, infatti, che in primis il degente può avere bisogno d'aiuto. ${ }^{10}$

\section{Conclusioni}

L'evento suicidio, sopravvenuto in un reparto ospedaliero, ha sempre un grave impatto emotivo su tutta l'équipe assistenziale, sia medica che infermieristica, che percepisce, dopo l'evento, un vissuto d'inadeguatezza, per non essere stata in grado di riconoscere i rischi per prevenire un tale esito fatale. Si rende pertanto necessaria una formazione specifica ad hoc per gli operatori sanitari, in particolare per il personale dei servizi di Emergenza e di Medicina Interna. ${ }^{11,12}$ Per un approfondimento di merito si rimanda specificamente al Corso organizzato dalla AUSL di Modena sulla prevenzione del suicidio. ${ }^{13}$

\section{Vagabondaggio}

Il termine vagabondaggio (wandering nella lingua inglese) definisce l'atteggiamento che presentano alcuni malati che tendono alla fuga e a vagare senza meta. ${ }^{14} \mathrm{I}$ pazienti ospedalizzati e/o istituzionalizzati affetti da demenza o altri tipi di disfunzione cognitiva sono a rischio di perdersi all'interno o all'esterno dell'ospedale o della struttura ospitante. ${ }^{15}$ Tra i fattori che possono accentuare la tendenza al vagabondaggio o ad un comportamento errante possono rientrare, ad esempio, le malattie che comportino un dolore cronico incessante refrattario alla terapia, la sete e la disidratazione, una sensazione soggettiva di abbandono e di solitudine, un senso di insicurezza, paura o di perdita (ad esempio la scomparsa o il cambio del caregiver di riferimento o le mancate visite di un familiare atteso), un trasferimento intra-interospedaliero, la percezione di un'atmosfera ostile ed estranea da parte degli astanti, uno stress sensoriale visivo o uditivo; una particolare intolleranza all' ambiente ospedaliero, irrequietezza e stati d'ansia con tachiprassia, agitazione psico-motoria, movimenti incontrollati, pulsione non orientata e disorientamento temporo-spaziale, la deprivazione sensoriale, l'effetto paradosso di psicofarmaci, precedenti iterative richieste di permesso di uscita con episodi di allontanamento spontaneo, spesso alla ricerca di droghe o alcool). Il comportamento del vagabondo è generalmente contrassegnato da quello di un soggetto affaticato, spesso trascurato e in condizioni igieniche precarie, con indumenti raffazzonati o malamente indossati e calzature inidonee - se non assenti -, confabulante, con una deambulazione incerta, non ben orientata in una direzione e con bisogno di camminare senza scopo o meta apparente, in un affaccendamento inutile non finalizzato. Camminare in modo persistente o incessante, con andatura incerta ed atteggiamento passivo, viene significativamente correlato alla tendenza alla fuga e ad esiti negativi del wandering. ${ }^{16}$ Le conseguenze del vagabondaggio possono essere finanche mortali, per esposizione ad eventi avversi di vario tipo, quali squilibri idro-elettrolitici, iper/ipotermia, congelamento, infezioni, cadute, fratture, politraumi e traumatismi stradali (da investimento da parte di veicoli) o ferroviari ed annegamento, anche in acque poco profonde. Una delle sfide più importanti nel fornire un'assistenza sicura ai pazienti propensi a vagabondaggio è garantire una supervisione intensiva dal momento del ricovero fino alla dimissione. Alcuni elementi salienti per affrontare il problema sono riportati nella Tabella 3.17-20

\section{Conclusioni}

Tutti gli ospedali dovrebbero avere una politica specifica finalizzata alla prevenzione del vagabondaggio e alla definizione delle possibili modalità da sviluppare - anche di tipo tecnologo - per la ricerca pro-attiva di pazienti ricoverati con problemi cognitivi che si sono perduti e non si trovano.

\section{Intrappolamento}

L'intrappolamento (entrapment nella lingua inglese) è definito come un evento che coinvolge un paziente intrappolato, impigliato o avviluppato nelle strutture del letto e degli effetti letterecci. ${ }^{21}$ Diverse condizioni di salute e caratteristiche del paziente sono state associate ad un aumentato rischio di intrappolamento del paziente nei letti d'ospedale. Queste includono disturbi cognitivi e di comunicazione, pazienti minuti e fragili, l'agitazione psico-motoria, un affaccendamento persistente, il dolore incontrollato, disfunzioni sfinteriche urinarie e/o fecali. Gli operatori sanitari dovrebbero effettuare una valutazione del paziente per selezionare i pazienti a rischio, onde monitorarli da vicino. I principali elementi clinico-assistenziali da considerare sono riportati nella Tabella 4.

Le zone del letto maggiormente a rischio sono rappresentate nella Figura $3 .^{22}$

Le zone indicate stanno alla base dell' $80 \%$ degli incidenti da intrappolamento riportati e per le quali la FDA fornisce indicazioni dimensionali specifiche. Le zone 1-3 rappresentano un rischio di intrappolamento della testa e la zona 4 del collo. Per impedire che il tronco, la testa e il collo restino intrappolati nella base del letto, i materassi devono coprire completamente la base del letto e resistere alle sollecitazioni del peso e 
dei movimenti del paziente. È necessario pertanto impedire i rischi di intrappolamento dovuti a spazi vuoti e aperture. I requisiti di sicurezza per le sponde laterali sono finalizzati a: i) ridurre il rischio dell'intrappola- mento potenziale del paziente tra la sponda e la testata, tra le due barre, tra la sponda e la rete; ii) incrementare la robustezza della sponda nel caso di urti laterali. Nel caso di sponde continue (o di lunghezza superiore ai $3 / 4$

Tabella 3. Elementi salienti per affrontare il problema del vagabondaggio. (Modificata da Rowe, 2008 ${ }^{17}$ ).

Obiettivo Considerazioni

Prima dell'evento: strategie di prevenzione

Garantire l'identificazione dei pazienti a rischio

Il malato dovrebbe sempre avere con sé un qualche documento d'identificazione (nome, cognome, indirizzo, reparto di degenza). In analogia a quanto proposto per i pazienti a rischio di caduta, l'impiego di braccialetti colorati (o di camici di colore dedicato, eventualmente con un'etichetta con i dati identificativi) può essere utile per il riconoscimento dei pazienti che richiedono una supervisione speciale. Indicatori della necessità di una supervisione intensiva potrebbero anche essere collocati sulle porte delle stanze o nella targa nominativa del paziente a capo del letto. Può essere utile disporre di fotografia recente del paziente a rischio di vagabondaggio

Coinvolgere i familiari e gli amici

I familiari e gli amici vanno incoraggiati a rimanere con il paziente durante il ricovero in ospedale. Un viso amico, una voce familiare e un approccio affettuoso possono ridurre l'agitazione e il senso di abbandono, supportando il programma assistenziale. Sarebbe opportuno facilitare la permanenza dei congiunti durante la degenza ospedaliera, fornendo servizi di comfort: stanze private, orari di visita aperti e prolungati, fornitura di comode sedie o lettini per il riposo, la disponibilità di servizio di caffetteria o di distributori automatici

Garantire un monitoraggio elettronico

Diversi sistemi di monitoraggio sono disponibili in commercio. Alcuni devono essere installati permanentemente nell'ambiente che deve essere controllato (ad esempio, nell'unità operativa in cui il paziente è ricoverato). Altri possono essere fatti indossare dai singoli pazienti: sono oggi disponibili trasmettitori portati al polso come un orologio, in cui il segnale trasmesso consente, con l'impiego di appositi ricevitori, di trovare la persona perduta. Questa tecnologia è talmente efficiente che la maggior parte delle persone che indossano questo dispositivo può essere trovate in 30 minuti. ${ }^{18}$ Tali sistemi si basano, in sostanza, sull'uso di software defined networking (SDN), Wireless LAN (WLAN), ovvero di dispositivi indossabili dai pazienti che consentono il monitoraggio dei soggetti a rischio ${ }^{19}$

Garantire un sorveglianza intensiva

Il personale infermieristico dovrebbe controllare tutti i pazienti a rischio di allontanarsi, anche con sistemi videosorveglianza e controllo a distanza. Per la sorveglianza può essere impiegato anche personale non retribuito o volontario, se disponibile, particolarmente quando ulteriori problemi di agitazione o confusione possono aumentare il rischio di allontanarsi

Prestare attenzione alle aree incustodite e ai momenti morti,

I pazienti sono a rischio di allontanarsi anche quando non si trovano nell'area di

in cui i livelli di controllo possono ridursi

degenza. In alcune fasi del ricovero il controllo del paziente può non essere direttamente garantito dal personale di reparto: ad esempio, nei trasferimenti interni, in zone di attesa per accertamenti diagnostici o per visite specialistiche $o$, in generale, in tutti i casi in cui il paziente potenzialmente vagabondo venga malauguratamente lasciato solo in una zona esterna all'unità di cura.Ė necessario creare in ospedale una cultura dei dipendenti atta a riconoscere tempestivamente (per comportamenti e sistemi di riconoscimento) i soggetti che tendono alla fuga

Implementare altre strategie utili

Bisognerebbe organizzare l'ambiente in modo tale che questi soggetti abbiano uno spazio sicuro dove poter vagare liberamente. Sarebbe opportuno inoltre bloccare l'accesso a luoghi non sicuri, impedire l'accesso ad ascensori, scale o porte di uscita dal reparto ${ }^{20}$

Dopo l'evento: strategie di gestione della fuga

La necessità di garantire una risposta rapida da parte dell'Ospedale

Al fine di facilitare una ricerca efficace, tutti gli ospedali dovrebbero avere una politica specifica sulla prevenzione del vagabondaggio e su modalità attive per la ricerca di pazienti con problemi cognitivi che si sono perduti 
rispetto al piano rete) i nuovi standard prevedono di utilizzare particolari strumenti per valutare il rischio di intrappolamento e le aree di maggior rischio. I requisiti da rispettare sulle sponde sono riportati in Tabella 5.
Oltre ai componenti del sistema letto, gli incidenti di intrappolamento del paziente possono essere correlati al materasso che, ad esempio, può essere di misura inadatta, incompatibile con le dimensioni del

Tabella 4. Elementi da valutare nel paziente per individuare i pazienti a rischio di intrappolamento.

- Status mentale del paziente (orientato, attento, confuso, sonnolento, soporoso, disorientato, inconscio, agitato, eccetera)

- Stato di mobilità del paziente (incapacità di deambulare in sicurezza da e verso la toilette; incapacità di trasferire in sicurezza tra il letto e la sedia a rotelle)

- Pazienti con autonomia motoria limitata, ad esempio con deficit neuro-motori o in grave sovrappeso

- Precedenti eventi indesiderati correlati all'allettamento prolungato

- Rischio di caduta del paziente (episodi di cadute precedenti, sovrappeso, semi-cosciente, paralisi parziale, convulsioni o spasmi, sedazione, deficit visivo, confusione)

- Rischio di lesioni da caduta (anziani minuti e fragili, osteoporosi, anticoagulanti, cute delicata, malattie vascolari, gravemente malati)

- Livello di ansia del paziente e di sopportazione di sponde al letto e dei mezzi di contenzione

- $\quad$ Urgenza e/o disfunzione minzionale/fecale

- $\quad$ Farmaci (effetto paradosso o rebound degli psicofarmaci)

Tabella 5. Principali requisiti necessari per garantire la sicurezza dei letti in ospedale.

- Distanza tra sponda e testata: meno di $60 \mathrm{~mm}$

- Distanza tra le assi della sponda: meno di $120 \mathrm{~mm}$

- Distanza tra piano rete e asse della sponda: meno di $120 \mathrm{~mm}$

- $\quad$ Distanza tra sponda e pediera: meno di $60 \mathrm{~mm}$ o maggiore di $318 \mathrm{~mm}$

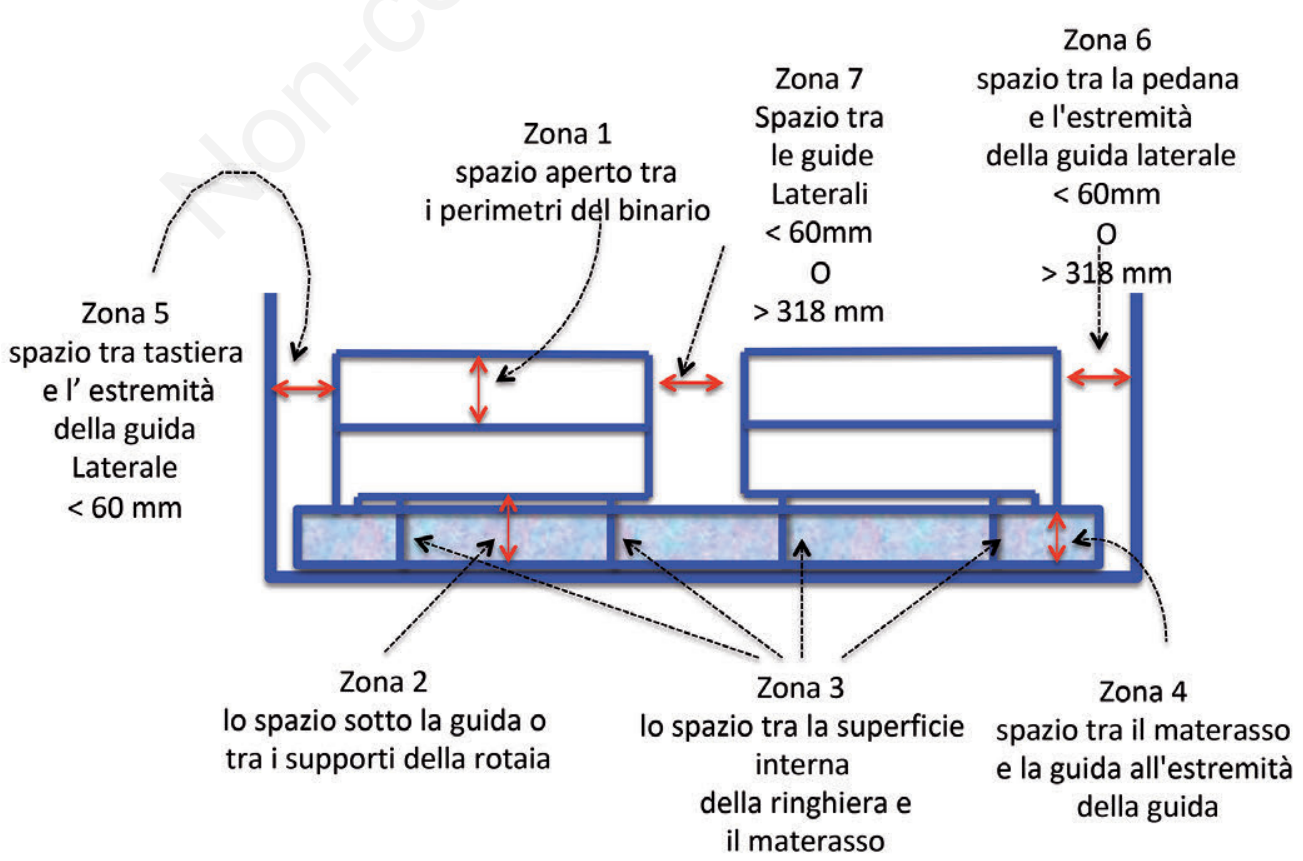

Figura 3. Le zone del letto a maggior rischio di intrappolamento. (Modificata da Haugh, 201422). 
letto, tanto da creare vuoti e spazi insicuri. I vecchi materassi, con il tempo, possono comprimersi, ridursi di volume e creare aperture pericolose. Per ulteriori dettagli si rimanda alla normativa UNI CEI EN 60601-2-52:2016 - Apparecchi elettromedicali Parte 2-52: Requisiti particolari per la sicurezza di base e le prestazioni essenziali dei letti medici. Altri riferimenti utili sono indicati da FDA. ${ }^{23-25}$ Purtroppo tali indicazioni sono rispettate in maniera assolutamente inadeguata. In uno studio effettuato in un ospedale per pazienti acuti solamente $1^{\prime} 8,3 \%$ dei letti presentava caratteristiche compatibili con le dimensioni standard di riferimento. ${ }^{22}$ Nelle Figure 4 e 5 sono rappresentate alcune delle situazioni più frequentemente osservabili in corso di intrappolamento.

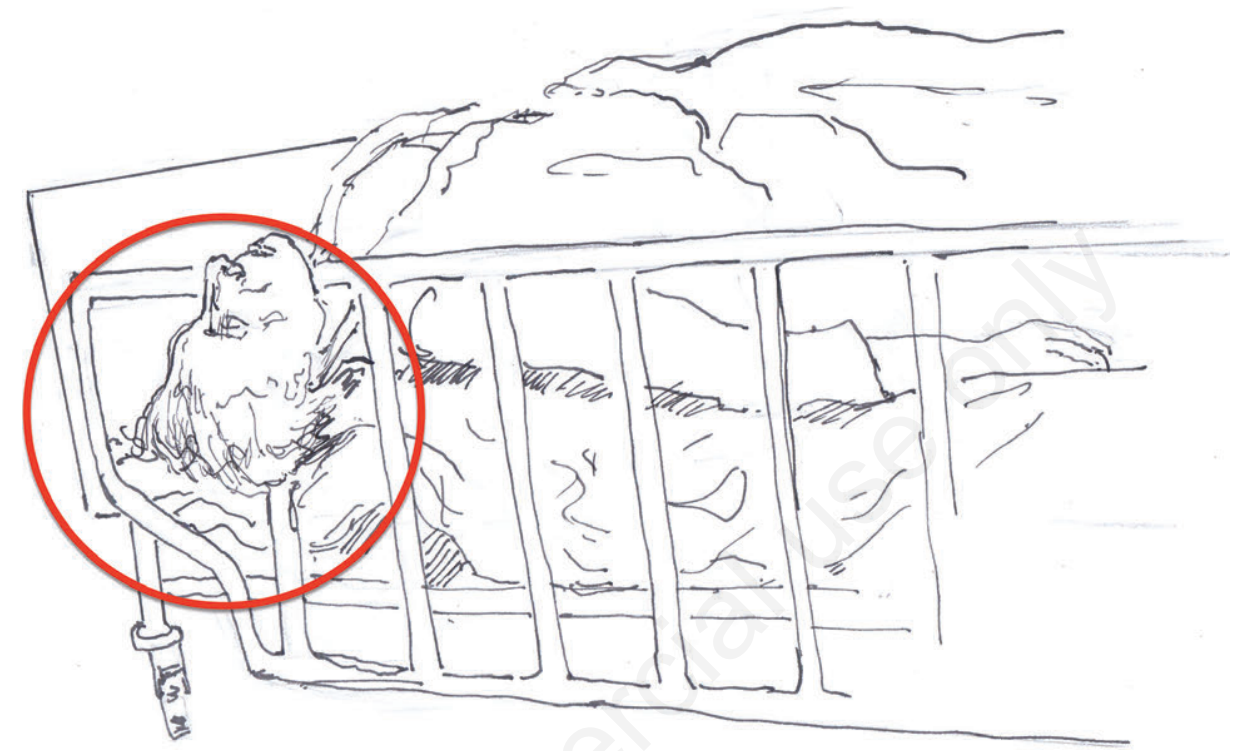

Figura 4. Intrappolamento - esempio 1.

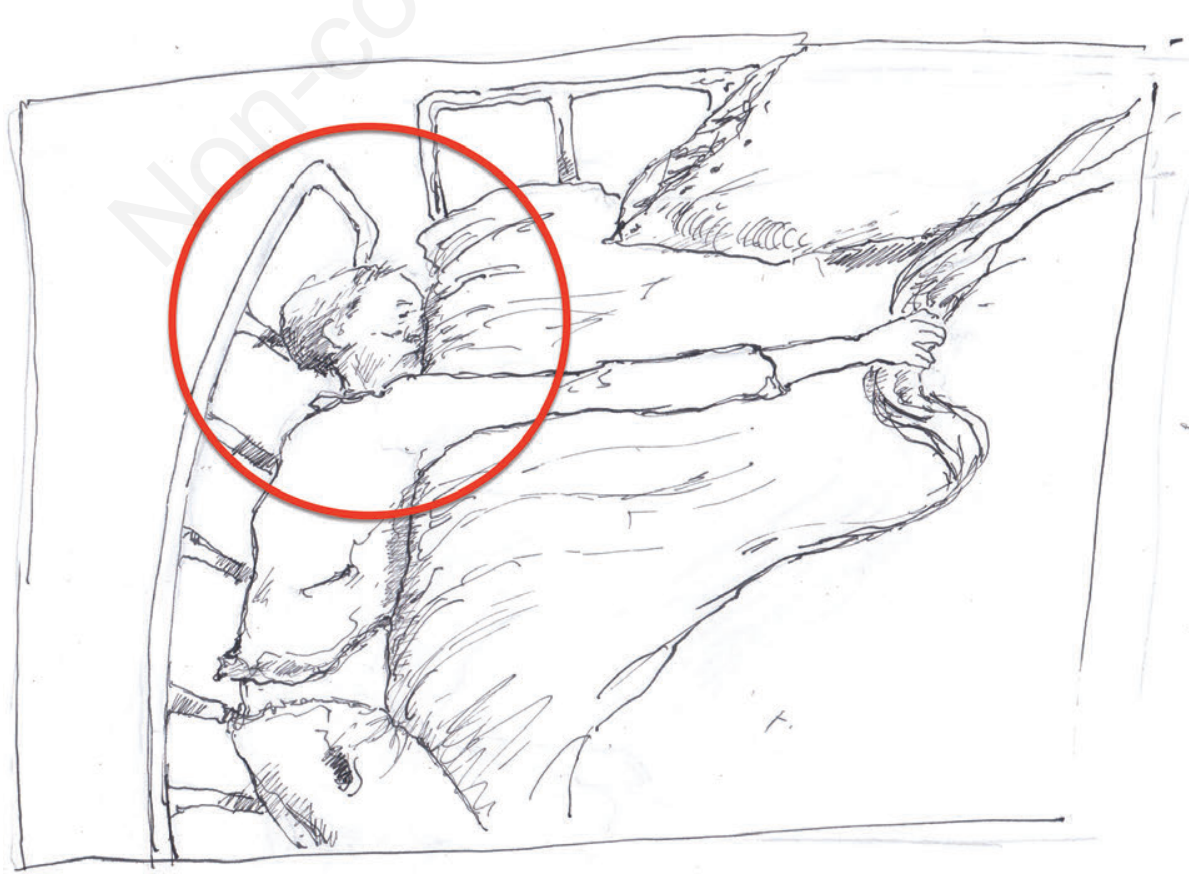

Figura 5. Intrappolamento - esempio 1. 


\section{Conclusioni}

Ogni ospedale dovrebbe attentamente considerare il problema dell'intrappolamento come uno dei potenziali rischi per i pazienti ricoverati. È necessaria una severa politica di controllo e monitoraggio sulla tipologia e la qualità dei letti disponibili nelle aree di degenza. Deve essere verificata la loro rispondenza alle normative vigenti e agli standard più aggiornati, secondo linee guida e le raccomandazioni disponibili, assicurando che vengano regolarmente espletate le istruzioni del produttore, sia nelle modalità di gestione che di manutenzione. ${ }^{26-29}$

\section{Bibliografia}

1. Quotidiano della Sanità 28 marzo 2013. Disponibile su: http://www.quotidianosanita.it/studi-e-analisi/articolo. php?articolo_id $=14178$

2. Ministero della Salute, Dipartimento della qualità, Direzione generale della programmazione sanitaria, dei livelli di assistenza e dei principi etici di sistema, ufficio III, Prevenzione del suicidio di paziente in ospedale, Raccomandazione n. 4, Ottobre 2006.

3. Regione Emilia Romagna, GdL coordinato da Piazza A, Raccomandazioni per la prevenzione delle condotte suicidarie in ospedale; 2011. Disponibile su: https://salute. regione.emilia-romagna.it/documentazione/leggi/regionali/linee-guida/raccomandazioni_prevenzione_suicidi_territorio.pdf

4. Nardi R. Relazione presentata al Corso Risk Management, La verità e il suo doppio, La gestione del rischio clinico nell'Azienda USL di Bologna, 2010.

5. Shneidman ES, Farberow NL, Litman RE. The Psychology of Suicide. New York: Science House; 1970.

6. O'Connell H, Chin AV, Cunningham C, Lawlor BA. Recent developments: Suicide in older people. BMJ 2004;329:895.

7. Nardi R, et al. Quaderni Italian Journal of Medicine 2015;3:e2:15.

8. Montgomery SA, Asberg M. New depression scale designed to be sensitive to change. Br J Psychiatry 1979;134:382-9.

9. Williams JBW, Kobak KA, Development and Reliability of a Structured Interview Guide for the MontgomeryAsberg Depression Rating Scale (SIGMA). Br J Psychiatr 2008;192/1:52-8.

10. Rossi S. Rischio suicidiario del paziente, linee guida e responsabilità della struttura sanitaria. Disponibile su: http://www.rivistaresponsabilitamedica.it/rischio-suicidiario-del-paziente-linee-guida-responsabilita-dellastruttura-sanitaria/

11. Palmieri G, Po S, Rigatelli M. Il rischio di suicidio nell'ospedale generale, II Congresso SICP ER, Bologna, Aula Magna Ospedale Maggiore, 24 novembre 2005.

12. Palmieri G, Forghieri M, Ferrari S, et al. Suicide intervention skills in health professionals: a multidisciplinary comparison. Arch Suicide Res 2008;12:232-7.

13. Pompili M. La prevenzione del suicidio, Corso di aggiornamento, 23 settembre 2009, Sala Grande Villa Boschetti corso Libertà S. Cesario s/P. (Mo). Disponibile su: http://www.ausl.mo.it/flex/cm/pages/ServeBLOB.php/L/I T/IDPagina/4689

14. Codemo A. Vagabondaggio ed affaccendamento. In: Gallina P, Corti MC. Disturbi comportamentali nella demenza Manuale per il Caregiver, 2007 ULSS 16 Padova, Cap. 6:28-30.

15. Cipriani G, et al. Wandering and dementia. Psychogeriatrics 2014;14:135-42.

16. Ali N, Luther SL, Volicer L, et al. Risk assessment of wandering behavior in mild dementia. Int J Geriatr Psychiatry 2016;31:367-74.

17. Rowe M. Wandering in Hospitalized Older Adults: wandering in patients with dementia, AJN, American Identifying risk is the first step in this approach to preventing. J Nursing 2008;108/10:62-70.

18. Progetto Lifesaver. Disponibile su: http://www.projectlifesaver.org/site

19. Varadharajan V et al. Secure Monitoring of Patients With Wandering Behavior in Hospital Environments. IEEE ACCESS 2018;6:11523-33.

20. Bertoncini F, Di Berardino S, Lo Burgio I, et al. La ricerca finalizzata in Medicina Interna: approfondimenti assistenziali dell'Associazione A.N.ì.M.O. Quad Int J Med 2019;7:1-62.

21. Health Canada, Recall and safety alerts, Hospital Beds - Risk of Patient Entrapment; April 2017. Disponibile su: https://healthycanadians.gc.ca/recall-alert-rappelavis/hc-sc/2017/62960a-eng.php

22. Haugh $J$ et al. High frequency of potential entrapment gaps in beds in an acute hospital. Age Ageing 2014;43:862-5.

23. Hospital Bed System Dimensional and Assessment Guidance to Reduce Entrapment - Guidance for Industry and FDA Staff. Available from: https://www. fda.gov/downloads/MedicalDevices/DeviceRegulationandGuidance/GuidanceDocuments/UCM072729.pdf

24. Clinical Guidance for the Assessment and Implementation of Bed Rails in Hospitals, Long Term Care Facilities and Home Care Settings.

25. HBSW Hospital Bed Safety Entrapment Kit Ordering Instructions; FDA and CPSC Letter to Manufacturers About Voluntary Standard Development For Adult Portable Bed Rails.

26. Guidance Document. Adult Hospital Beds: Patients Entrapment Hazards, Side Rail Latching Reliability, and Other Hazards. Ottawa (ON): Health Canada; 2008 Mar 17.

27. Hospital bed safety. Ottawa (ON): Health Canada; 2015 Feb 20.

28. Hospital Beds - Risk of Patient Entrapment (Update) Notice to Hospitals. Ottawa (ON): Health Canada; 2012 Aug 1.

29. Risk of Entrapment of Patients in Hospital Beds - Notice to Hospitals. Ottawa (ON): Health Canada; 2009 Oct 20. 


\title{
Gestione ricoveri in appoggio e rischio clinico
}

\author{
Micaela La Regina, ${ }^{1}$ Francesca Guarneri, ${ }^{2}$ Tommaso Bellandi, ${ }^{3}$ Riccardo Tartaglia,${ }^{4}$ Antonio Brucato,${ }^{5}$ \\ Dario Manfellotto ${ }^{6}$ \\ ${ }^{1}$ SS Risk Management, ASL5 Liguria; ${ }^{2}$ Attività Sanitarie di Comunità, Azienda USL Toscana Nord Ovest, Pisa; \\ ${ }^{3}$ UOC Sicurezza del paziente, Azienda USL Toscana Nord Ovest, Pisa; ${ }^{4}$ Centro di Gestione Rischio Clinicio e Sicurezza del \\ paziente della Regione Toscana, Firenze; ${ }^{5}$ Dipartimento di Scienze Biomediche e Cliniche, Università degli Studi di Milano, \\ Ospedale Fatebenefratelli; 'U.O.C. Medicina Interna. Osp. "S. Giovanni Calibita” Isola Tiberina - Roma, Italia
}

\section{Premessa}

Il progressivo taglio dei posti letto (45.000 in tutta Italia dal 2000 al 2009, dati di Quotidianosanità), non accompagnato a un potenziamento della Medicina del territorio e unito a una concezione ospedalo-centrica della salute da parte dei cittadini e all'aumento delle dimissioni difficili per fragilità sociosanitaria, conduce da alcuni anni, a indisponibilità di posti letto, per lo più in area medica, con conseguente ricorso da parte del Pronto Soccorso (PS) al ricovero fuori reparto. Tale fenomeno inizialmente concentrato nei mesi invernali, negli ultimi anni sta perdendo il carattere stagionale, divenendo spia di gravi e profonde disfunzioni organizzative.

Il ricovero fuori reparto, anche detto appoggio o deposito o, nella letteratura internazionale, outlier, outlying hospital in-patient, overflow, sleep-out o boar$d e r,{ }^{1-3}$ si configura quando, per mancanza di posti letto liberi nel Reparto di Competenza Nosologica (RCN), il PS invia il paziente in altro reparto con posto letto libero. In tal caso, la gestione clinica è in carico al RCN e l'assistenza infermieristica e di supporto al reparto cosiddetto di Assistenza (RA). Un esempio è il paziente con polmonite ricoverato a carico della Medicina Interna, ma fisicamente collocato in Ortopedia. Vi sono realtà dove il paziente viene ricoverato in carico al reparto che lo ospita, mentre l'equipe del RCN interviene come consulente. Questa condizione è anche peggiore perché il paziente non è preso in carico

Corrispondente: Micaela La Regina, S.S. Risk Management, ASL 5 Spezzino, via Fazio 30, 19121 La Spezia, Italia. Tel.: +39.0187533782.

E-mail: micaela.laregina@asl5.liguria.it

Articolo pubblicato secondo la Creative Commons Attribution NonCommercial 4.0 License (CC BY-NC 4.0).

${ }^{\circ}$ Copyright: the Author(s), 2019

Licensee PAGEPress, Italy

QUADERNI - Italian Journal of Medicine 2019; 7(6):105-110 effettivamente dall'equipe di competenza e il controllo evolutivo è affidato a una equipe che ha meno familiarità nella gestione (es. equipe chirurgica - paziente con scompenso cardiaco).

\section{Breve revisione della letteratura}

I Ricoveri fuori Reparto (RfR) sono tipici dei paesi con sistema sanitario pubblico, ammontano a circa il $7-8 \%,{ }^{1}$ sono conseguenza dei tagli ai posti letto e stanno assumendo caratteristiche drammatiche per dimensioni e sostenibilità, nonché per le ricadute negative sulla qualità e sicurezza delle cure e sulla soddisfazione di pazienti e operatori sanitari.

In letteratura, le poche evidenze disponibili, seppur di bassa qualità metodologica, dimostrano: i) tendenza al prolungamento della degenza per i $\mathrm{RfR}^{1,4-6}$ e aumento non costante della mortalità (Survey FADOI $R i$ coveri sovrannumerari $2018^{4,5}$ ) e delle riammissioni a 30 giorni; 4,5 gli autori attribuiscono il non costante aumento della mortalità alla precauzione di talune organizzazioni di collocare fuori reparto i pazienti meno gravi e l'aumento della durata della degenza a: ritardo fra arrivo del paziente e visita medica, contatti insufficienti con il RCN, ridotta capacità del RA di rispondere ai bisogni del paziente per diversa specializzazione, eventuali maggiori complicanze nosocomiali; ${ }^{1}$ ii) maggior numero di chiamate al sistema di soccorso intraospedaliero per i RfR; ${ }^{7-11}$ iii) maggiori competenze nella gestione dei pazienti con trauma negli infermieri dei reparti di competenza ${ }^{12}$ iv) riduzione del grado di soddisfazione di pazienti e operatori con timori reciproci circa la qualità e sicurezza delle cure, ${ }^{2,3} \mathrm{v}$ ) che essere nel letto giusto nel momento giusto migliori gli esiti dei pazienti con stroke, ustioni, asma ed emorragie gastrointestinali; $;^{7-10} \mathrm{vi}$ ) che alcuni interventi (facilitazione delle dimissioni o quick and sick ward con degenze di 2-3 giorni) potrebbero ridurre il fenomeno degli RfR; ${ }^{13,14}$ vii) che i pazienti fuori reparto sono più vulnerabili ai rischi $;{ }^{15}$ viii) che rappresentano un carico di lavoro aggiuntivo anche per gli spostamenti dei medici fra i reparti. ${ }^{16}$

Non esistono invece studi che abbiano misurato 
l'incidenza di eventi avversi nei ricoveri fuori reparto. Lo studio FADOI - SISIFO (NCT03651414) Safety Issues and SurvIval For medical Outliers partito to scorso autunno colmerà tale gap conoscitivo.

\section{Analisi dei rischi e azioni di miglioramento}

Lepage et al. ${ }^{15}$ hanno condotto una Failure Mode Effects Analysis (FMEA) in un ospedale francese per valutare i rischi corsi dagli outlier a partire dal dipartimento di emergenza e per tutto il ricovero, fino alla dimissione, individuando come prioritari il rischio di essere visitati ogni giorno da un medico diverso, di non essere noti al reparto di competenza nosologica e la perdita di informazioni cliniche.

Tuttavia, risentendo molto dell'organizzazione locale, i rischi individuati da Lepage et al. ${ }^{15}$ non sono direttamente trasferibili alla nostra realtà italiana.

Di seguito (Tabella 1) riportiamo i risultati di un

Tabella 1. Rischi dei ricoveri fuori reparto, raccomandazioni per la prevenzione/riduzione, fattori abilitanti e ostacolanti la raccomandazione

\begin{tabular}{|c|c|c|c|c|}
\hline Condizione & $\begin{array}{l}\text { Effetti negativi } \\
\text { sul processo di cura }\end{array}$ & Raccomandazioni & Fattori abilitanti & Ostacoli \\
\hline $\begin{array}{l}\text { I medici del RCN } \\
\text { visitano i RfR a rotazione }\end{array}$ & $\begin{array}{l}\text { Discontinuità di cura } \\
\text { Difficoltà } \\
\text { nell'instaurazione } \\
\text { di un rapporto } \\
\text { medico-paziente } \\
\text { efficace }\end{array}$ & $\begin{array}{l}\text { Assicurare che il RfR } \\
\text { sia visitato dallo stesso } \\
\text { medico, salvo assenze } \\
\text { per guardie notturne } \\
\text { o ferie } \\
\text { Consegne in formato } \\
\text { elettronico, scritte ed } \\
\text { aggiornate in tempo reale }\end{array}$ & $\begin{array}{l}\text { Risparmio di tempo } \\
\text { nelle visite successive } \\
\text { Comunicazione chiara, } \\
\text { tempestiva ed efficace } \\
\text { Gestione sicura anche } \\
\text { a distanza } \\
\text { Supporto alle decisioni } \\
\text { in caso di urgenza }\end{array}$ & $\begin{array}{l}\text { Resistenza dei medici a recarsi } \\
\text { tutti i giorni fuori reparto } \\
\text { Ulteriore carico di lavoro }\end{array}$ \\
\hline $\begin{array}{l}\text { Il RfR è visitato una } \\
\text { volta al giorno, } \\
\text { salvo urgenze }\end{array}$ & Perdita di informazioni & $\begin{array}{l}\text { Trasferire in reparto il } \\
\text { paziente fuori reparto } \\
\text { il prima possibile }\end{array}$ & $\begin{array}{l}\text { Riduzione delle visite } \\
\text { fuori reparto } \\
\text { Minore perdita } \\
\text { di informazioni }\end{array}$ & $\begin{array}{l}\text { Disponibilità di posti letto } \\
\text { Difficoltà nei trasporti interni } \\
\text { negli ospedali a padiglioni }\end{array}$ \\
\hline $\begin{array}{l}\text { Il RfR è visitato spesso } \\
\text { dopo i pazienti del } \\
\text { reparto }\end{array}$ & $\begin{array}{l}\text { Ritardo nella presa in } \\
\text { carico } \\
\text { Ritardo diagnostico/ } \\
\text { terapeutico }\end{array}$ & $\begin{array}{l}\text { Visitare i pazienti in } \\
\text { base alla gravità o } \\
\text { iniziando dai RfR } \\
\text { Visitare il paziente } \\
\text { appena ricoverato fuori } \\
\text { reparto entro } 1 \text { ora }\end{array}$ & $\begin{array}{l}\text { Aumento della sicurezza } \\
\text { delle cure }\end{array}$ & $\begin{array}{l}\text { Resistenza dei medici a recarsi } \\
\text { fuori reparto }\end{array}$ \\
\hline $\begin{array}{l}\text { Documentazione (STU) } \\
\text { talora differente dal RCN }\end{array}$ & $\begin{array}{l}\text { Possibili errori } \\
\text { terapeutici }\end{array}$ & $\begin{array}{l}\text { Controllare la scheda di } \\
\text { terapia con il personale } \\
\text { del RA }\end{array}$ & Prevenzione errori & $\begin{array}{l}\text { Indisponibilità del personale del } \\
\text { RA }\end{array}$ \\
\hline Mancata notifica al RCN & $\begin{array}{l}\text { Ritardi/omissioni } \\
\text { diagnostichee/o } \\
\text { terapeutiche }\end{array}$ & $\begin{array}{l}\text { Comunicazione telefonica } \\
\text { obbligatoria dal PS } \\
\text { al RCN } \\
\text { Verifica da parte del RA } \\
\text { Lavagna dei RfR nel RCN, } \\
\text { verificata periodicamente } \\
\text { Introduzione cartella } \\
\text { clinica informatizzata, } \\
\text { dove non presente }\end{array}$ & $\begin{array}{l}\text { Cure tempestive ed } \\
\text { appropriate } \\
\text { Prevenzione dei conflitti }\end{array}$ & $\begin{array}{l}\text { Aggravio di lavoro per il PS } \\
\text { Opposizione del RCN durante la } \\
\text { telefonata }\end{array}$ \\
\hline $\begin{array}{l}\text { Mancata/ritardata } \\
\text { comunicazione } \\
\text { dell'appuntamento o } \\
\text { inadeguata preparazione } \\
\text { all'esame }\end{array}$ & Vedi sopra & $\begin{array}{l}\text { Indicazione del RA nelle } \\
\text { richieste diagnostiche }\end{array}$ & Vedi sopra & $\begin{array}{l}\text { Consuetudini radicate } \\
\text { Difficoltà nelle modifiche a } \\
\text { programmi informatici già in uso }\end{array}$ \\
\hline $\begin{array}{l}\text { Prolungamento durata } \\
\text { giro visita e sovraccarico } \\
\text { imprevedibile di lavoro } \\
\text { per il RCN }\end{array}$ & $\begin{array}{l}\text { Riduzione della qualità } \\
\text { delle cure } \\
\text { Possibili errori } \\
\text { diagnostici o terapeutici }\end{array}$ & $\begin{array}{l}\text { Non utilizzare il RfR } \\
\text { come modalità routinaria } \\
\text { Valutare sempre prima } \\
\text { percorsi alternativi al } \\
\text { ricovero } \\
\text { Distribuire equamente i } \\
\text { RfR fra discipline affini }\end{array}$ & $\begin{array}{l}\text { Riduzione attività fuori } \\
\text { reparto } \\
\text { Riduzione sovraccarico } \\
\text { di lavoro } \\
\text { Aumento della sicurezza } \\
\text { di pazienti e operatori }\end{array}$ & $\begin{array}{l}\text { Congestione del PS } \\
\text { Resistenza dei pazienti } \\
\text { Privilegio di alcune UO di non } \\
\text { avere RfR (per consuetudine, } \\
\text { alleanze, timori, ecc) }\end{array}$ \\
\hline $\begin{array}{l}\text { Giro-visita aggiuntivo } \\
\text { e non formalizzato } \\
\text { per il RA }\end{array}$ & $\begin{array}{l}\text { Cattivo passaggio di } \\
\text { consegne }\end{array}$ & $\begin{array}{l}\text { Individuazione ad opera } \\
\text { del coordinatore } \\
\text { infermieristico del RA } \\
\text { di un infermiere dedicato } \\
\text { ai RfR, per ogni turno }\end{array}$ & $\begin{array}{l}\text { Migliore conoscenza e } \\
\text { assistenza ai RfR }\end{array}$ & $\begin{array}{l}\text { Difficoltà a individuare } \\
\text { l'infermiere adatto/disponibile } \\
\text { in ogni turno }\end{array}$ \\
\hline
\end{tabular}


Tabella 1. Segue dalla pagina precedente

\begin{tabular}{|c|c|c|c|c|}
\hline Condizione & $\begin{array}{l}\text { Effetti negativi } \\
\text { sul processo di cura }\end{array}$ & Raccomandazioni & FattoriAbilitanti & Ostacoli \\
\hline $\begin{array}{l}\text { Minore familiarità } \\
\text { del RA con la tipologia } \\
\text { dei RfR }\end{array}$ & $\begin{array}{l}\text { Mancato precoce } \\
\text { riconoscimento dei segni } \\
\text { di deterioramento } \\
\text { (soprattutto dove non } \\
\text { utilizzati score prognostici } \\
\text { come MEWS/NEWS) }\end{array}$ & $\begin{array}{l}\text { Introduzione del MEWS/ } \\
\text { NEWS dove non presente } \\
\text { Indicazione al personale } \\
\text { infermieristico del RA } \\
\text { dei parametri da } \\
\text { monitorare e dei segnali } \\
\text { di allarme (da riportare } \\
\text { in cartella clinica) }\end{array}$ & $\begin{array}{l}\text { Percezione di maggiore } \\
\text { sicurezza per il personale } \\
\text { del RA }\end{array}$ & Sovraccarico di lavoro per il RA \\
\hline $\begin{array}{l}\text { Paziente vissuto come } \\
\text { temporaneo } \\
\text { Mancata acquisizione } \\
\text { di farmaci/ } \\
\text { apparecchiature } \\
\text { mancanti }\end{array}$ & $\begin{array}{l}\text { Ritardi/omissioni/errori } \\
\text { terapeutici }\end{array}$ & $\begin{array}{l}\text { Verifica immediata della } \\
\text { disponibilità di farmaci o } \\
\text { apparecchiature necessari } \\
\text { ed eventuale } \\
\text { approvvigionamento } \\
\text { Formazione sui farmaci } \\
\text { indispensabili (poster } \\
\text { nelle infermerie) }\end{array}$ & $\begin{array}{l}\text { Aumento sicurezza di } \\
\text { pazienti e operatori } \\
\text { Acquisizione di nuove } \\
\text { conoscenze e } \\
\text { competenze }\end{array}$ & $\begin{array}{l}\text { Vedi sopra } \\
\text { Scarsa propensione alla } \\
\text { formazione }\end{array}$ \\
\hline $\begin{array}{l}\text { Minore conoscenza da } \\
\text { parte del RA di farmaci/ } \\
\text { apparecchiature per } \\
\text { il RfR }\end{array}$ & Come sopra & $\begin{array}{l}\text { Riduzione al minimo } \\
\text { dell'uso di apparecchiature } \\
\text { non familiari al RA } \\
\text { Se indispensabili, chiara } \\
\text { illustrazione di } \\
\text { funzionamento, allarmi e } \\
\text { monitoraggio } \\
\text { Supporto da parte del } \\
\text { personale del RCN }\end{array}$ & $\begin{array}{l}\text { Condizioni cliniche } \\
\text { favorevoli }\end{array}$ & Condizioni cliniche sfavorevoli \\
\hline $\begin{array}{l}\text { Richieste esami e } \\
\text { provette preparate dal } \\
\text { RCN e prelievo eseguito } \\
\text { dal RA }\end{array}$ & Errori di identificazione & $\begin{array}{l}\text { Evitare che il RCN prepari } \\
\text { le richieste per gli esami e } \\
\text { le provette per i RfR }\end{array}$ & $\begin{array}{l}\text { Minore sovraccarico di } \\
\text { lavoro del RCN }\end{array}$ & Resistenza del RA \\
\hline $\begin{array}{l}\text { Richieste esami preparate } \\
\text { dal RA con omissione di } \\
\text { esami non noti }\end{array}$ & $\begin{array}{l}\text { Omissioni/errori/ritardi } \\
\text { diagnostici }\end{array}$ & $\begin{array}{l}\text { Non omettere la richiesta } \\
\text { di esami che non si } \\
\text { conoscono, ma consultare } \\
\text { il Laboratorio analisi } \\
\text { o il RCN }\end{array}$ & $\begin{array}{l}\text { Cure tempestive e } \\
\text { appropriate }\end{array}$ & Sovraccarico di lavoro del RA \\
\hline Condizione & $\begin{array}{l}\text { Effetti negativi } \\
\text { sul paziente }\end{array}$ & Raccomandazioni & Fattori abiitanti & Ostacoli \\
\hline $\begin{array}{l}\text { Percezione di minore } \\
\text { sicurezza e qualità } \\
\text { delle cure }\end{array}$ & $\begin{array}{l}\text { Discomfort del paziente } \\
\text { e/o del caregiver }\end{array}$ & $\begin{array}{l}\text { Tranquillizzare il paziente } \\
\text { e i familiari che le cure } \\
\text { ricevute fuori reparto } \\
\text { sono altrettanto efficaci } \\
\text { e sicure } \\
\text { Fornire supporto } \\
\text { premuroso e puntuale }\end{array}$ & $\begin{array}{l}\text { Empatia del personale } \\
\text { medico-infermieristico }\end{array}$ & $\begin{array}{l}\text { Scarsa empatia del personale } \\
\text { medico-infermieristico }\end{array}$ \\
\hline $\begin{array}{l}\text { Difficoltà nel reperire } \\
\text { in tempi successivi } \\
\text { il medico di riferimento }\end{array}$ & $\begin{array}{l}\text { Ritardo nelle } \\
\text { informazioni sul } \\
\text { decorso }\end{array}$ & $\begin{array}{l}\text { Il nome del medico di } \\
\text { riferimento deve essere } \\
\text { chiaramente riportato } \\
\text { in cartella clinica e il RA } \\
\text { deve disporre di un suo } \\
\text { recapito telefonico diretto }\end{array}$ & $\begin{array}{l}\text { Presa in carico efficace } \\
\text { Affidabilità del medico } \\
\text { di riferimento }\end{array}$ & $\begin{array}{l}\text { Timore di chiamate eccessive e } \\
\text { non motivate }\end{array}$ \\
\hline Prolungamento degenza & $\begin{array}{l}\text { Maggiore esposizione a } \\
\text { complicanze } \\
\text { nosocomiali }\end{array}$ & $\begin{array}{l}\text { Impostare l'iter } \\
\text { diagnostico-terapeutico } \\
\text { e il programma } \\
\text { di dimissione } \\
\text { fin dall'ingresso }\end{array}$ & $\begin{array}{l}\text { Cure tempestive } \\
\text { e efficaci }\end{array}$ & Valutazione non collegiale \\
\hline Condizione & $\begin{array}{l}\text { Effetti negativi } \\
\text { sul sistema }\end{array}$ & Raccomandazioni & Fattori abiitanti & Ostacoli \\
\hline $\begin{array}{l}\text { Occupazione letti } \\
\text { chirurgici }\end{array}$ & $\begin{array}{l}\text { Ritardo chirurgia di } \\
\text { elezione }\end{array}$ & $\begin{array}{l}\text { Rimodulare, in base ai } \\
\text { volumi di attività, la } \\
\text { distribuzione dei letti fra } \\
\text { area medica e chirurgica e, } \\
\text { solo se appropriato, } \\
\text { ampliare il numero dei posti } \\
\text { letto medici con } \\
\text { adeguamento dell'organico }\end{array}$ & $\begin{array}{l}\text { Riduzione dei RfR } \\
\text { Incremento dell'organico } \\
\text { medico }\end{array}$ & $\begin{array}{l}\text { Resistenza della dirigenza } \\
\text { chirurgica } \\
\text { Riduzione attività chirurgica } \\
\text { più remunerativa }\end{array}$ \\
\hline
\end{tabular}


Tabella 1. Segue dalla pagina precedente

\begin{tabular}{|c|c|c|c|c|}
\hline Condizione & $\begin{array}{l}\text { Effetti negativi } \\
\text { sul sistema }\end{array}$ & Raccomandazioni & Fattori abiitanti & Ostacoli \\
\hline Eccesso di RfR & $\begin{array}{l}\text { Cattiva reputazione della } \\
\text { struttura ospedaliera } \\
\text { Riduzione efficacia e } \\
\text { efficienza }\end{array}$ & $\begin{array}{l}\text { Condivisione a mezzo } \\
\text { intranet di report sui RfR } \\
\text { (numero giornaliero per } \\
\text { UO e trend) } \\
\text { Alert sul numero dei RfR } \\
\text { nel cruscotto di bed } \\
\text { management o nel } \\
\text { software di pronto soccorso } \\
\text { Non consentire l'utilizzo } \\
\text { come reparto di assistenza } \\
\text { di reparti che abitualmente } \\
\text { hanno ricoveri fuori reparto } \\
\text { Evitare riduzione di posti } \\
\text { letto per i reparti abitualmer } \\
\text { con RfR (mediante } \\
\text { accorpamenti, altro) } \\
\text { Adoperarsi, in tutti i modi } \\
\text { per ridurre i RfR, a livello d } \\
\text { direzione strategica }\end{array}$ & $\begin{array}{l}\text { Miglioramento immagine } \\
\text { Miglioramento benessere } \\
\text { lavorativo } \\
\text { dipendenti } \\
\text { Miglioramento sicurezza } \\
\text { e qualità delle cure } \\
\text { Attenzione alla front-line } \\
\text { Capacità decisionale } \\
\text { Umanizzazione delle cure } \\
\text { di } \\
\text { nte }\end{array}$ & $\begin{array}{l}\text { Clima interno di scarsa } \\
\text { collaborazione } \\
\text { Scarsa attenzione della } \\
\text { dirigenza alla front-line } \\
\text { Difficoltà nelle modifiche a } \\
\text { programmi informatici già in uso }\end{array}$ \\
\hline Condizione & $\begin{array}{l}\text { Effetti negativi } \\
\text { sugli operatori }\end{array}$ & Raccomandazioni & Fattori abilitanti & Ostacoli \\
\hline Presenza di RfR & $\begin{array}{l}\text { Sovraccarico di lavoro } \\
\text { Percezione di ridotta } \\
\text { qualità e sicurezza } \\
\text { delle proprie cure }\end{array}$ & $\begin{array}{l}\text { Supportare } \\
\text { organizzativamente le } \\
\text { equipe coinvolte } \\
\text { (es. compensare } \\
\text { rapidamente o prevenire } \\
\text { carenze di organico, } \\
\text { affiancare personale } \\
\text { amministrativo, } \\
\text { informatizzazione) }\end{array}$ & $\begin{array}{l}\text { Riduzione del carico di } \\
\text { lavoro } \\
\text { Miglioramento benessere } \\
\text { lavorativo } \\
\text { Miglioramento di } \\
\text { sicurezza e qualità delle } \\
\text { cure }\end{array}$ & $\begin{array}{l}\text { Mancato supporto delle Risorse } \\
\text { Umane } \\
\text { Difficoltà nell'ottenere } \\
\text { l'assenso per eventuali } \\
\text { assunzioni } \\
\text { Costi del personale aggiuntivo }\end{array}$ \\
\hline
\end{tabular}

Tabella 2. Interventi per la riduzione/abolizione dei ricoveri fuori reparto e relativi fattori abilitanti e ostacolanti

\begin{tabular}{lll}
\hline Intervento multicomponente & Fattori abilitanti & Ostacoli \\
\hline $\begin{array}{l}\text { Interventi a breve termine } \\
\text { Revisione dell'allocazione delle risorse così da garantire, } \\
\text { in area medica, ogni giorno almeno 1 medico ogni 10-12 }\end{array}$ & $\begin{array}{l}\text { Riduzione e maggiore costanza del } \\
\text { carico di lavoro }\end{array}$ & $\begin{array}{l}\text { Difficoltà alla messa in comune di } \\
\text { risorse fra discipline affini }\end{array}$ \\
$\begin{array}{ll}\text { (eccezionalmente 15) pazienti, anche con la messa in } \\
\text { comune di risorse fra discipline affini (es. medicina interna }\end{array}$ & & $\begin{array}{l}\text { Resistenza a lavorare secondo nuovi } \\
\text { modelli organizzativi }\end{array}$
\end{tabular}

e geriatria). Il risultato è un maggior numero di dimissioni in sicurezza al giorno

Riduzione degenza media in area medica mediante soluzioni Solida organizzazione del quali esami in post-dimissione, attivazione precoce del percorso per le dimissioni difficili, riduzione degenza medi presso le strutture sanitarie territoriali, snellimento liste di attesa per diagnostica a favore dei ricoverati ad es. istituzione discharge room follow-up post-dimissione territoriali Organizzazione flessibile Buona recettività territoriale Organico sufficiente nei servizi di diagnostica o nelle strutture

Buona organizzazione territoriale

Organico del RCN sufficiente

Disponibilità di discharge room e pazienti adatti

Disponibilità del sistema di trasporto a domicilio e di pazienti o familiari

Affidabilità nella gestione ambulatoriale da parte dei medici dei reparti

Disponibilità di spazi e risorse

(6/7 giorni, ore 8-14) con unità dedicata dove possibile, anche in condivisione con discipline affini

Prevenzione carenze di organico o affiancamento di personale amministrativo nei reparti con RfR
Miglioramento del benessere lavorativo
Pazienti o familiari non complianti

Resistenza dei medici

Ridotta recettività territoriale

Carenza di organico nei servizi

diagnostici o nelle strutture territoriali

Cattiva organizzazione territoriale

Carenza di organico RCN

Organizzazione carente

Limiti strutturali

Mancanza di personale per la vigilanza

in discharge room

Mancata disponibilità del sistema di trasporto o di pazienti o dei familiari

Difficoltà alla messa in comune di risorse Carenza di organico

Mancato supporto da parte della SC

Risorse Umane

Difficoltà nell'autorizzazione

all'assunzione del personale Costi

Continua nella pagina seguente 
Tabella 2. Segue dalla pagina precedente

\begin{tabular}{lll}
\hline Intervento multicomponente & Fattori abilitanti & Ostacoli \\
\hline $\begin{array}{l}\text { Co-gestione della fase di ricovero fra PS e reparti di area } \\
\text { medica per migliorare l'appropriatezza di ricovero e la } \\
\text { distribuzione dei RfR fra discipline affini }\end{array}$ & $\begin{array}{l}\text { Buoni rapporti con PS } \\
\text { Buona reputazione dei medici addetti } \\
\text { ai rapporti con PS } \\
\text { Affidabilità nella gestione } \\
\text { ambulatoriale }\end{array}$ & $\begin{array}{l}\text { Sensazione di limitazione del proprio } \\
\text { giudizio clinico per i medici del PS } \\
\text { Poca affidabilità della gestione } \\
\text { ambulatoriale }\end{array}$ \\
\hline $\begin{array}{l}\text { Interventi a medio termine } \\
\begin{array}{l}\text { Percorsi diagnostico-terapeutici evidence-based per i DRG } \\
\text { a rischio di inappropriatezza }\end{array}\end{array}$ & $\begin{array}{l}\text { Buoni rapporti con MMG e/o con gli } \\
\text { altri specialisti ospedalieri }\end{array}$ & $\begin{array}{l}\text { Rapporti scadenti con MMG e/o con } \\
\text { altri specialisti ospedalieri } \\
\text { Scarsa confidenza con le linee guida }\end{array}$ \\
\hline $\begin{array}{l}\text { Interventi a lungo termine } \\
\begin{array}{l}\text { Formazione sull'appropriatezza di ricovero } \\
\text { Fimodulazione, in base ai volumi di attività degli ultimi }\end{array}\end{array}$ & $\begin{array}{l}\text { Riduzione/abolizione dei ricoveri } \\
\text { fuori reparto }\end{array}$ & $\begin{array}{l}\text { Resistenze della dirigenza di area } \\
\text { chirurgica }\end{array}$ \\
$\begin{array}{l}\text { S anni, della distribuzione dei letti fra area medica e } \\
\text { chirurgica }\end{array}$ & $\begin{array}{l}\text { Attitudine all'aggiornamento } \\
\text { formazione }\end{array}$ &
\end{tabular}

focus group condotto con alcuni operatori (medici e infermieri) di reparti di medicina interna italiani sui possibili rischi corsi dal paziente, dagli operatori e dall'azienda in presenza di ricoveri fuori reparto, unitamente alle raccomandazioni per ridurne o prevenirne l'impatto negativo e, non ultimi i fattori abilitanti e ostacolanti l'implementazione della raccomandazione.

Le raccomandazioni potrebbero essere incluse in una Pratica per la Sicurezza da diffondere a livello aziendale per il controllo dei rischi dei ricoveri fuori reparto.

Data la numerosità e la severità di taluni rischi e la necessità per le organizzazioni di garantire cure eque, sicure e di qualità, l'azienda dovrebbe adoperarsi per la riduzione/abolizione dei ricoveri fuori reparto. L'analisi delle cause condotta con il medesimo focus group ha evidenziato cause molteplici e variabili da organizzazione ad organizzazione, pertanto l'intervento non può che essere multicomponente e non può prescindere da una accurata analisi del contesto locale. La Tabella 2 riassume i possibili interventi scaturiti dal focus group, distinti in interventi a breve, medio e lungo termine, insieme ai relativi fattori abilitanti e ostacolanti.

\section{Conclusioni}

I ricoveri fuori reparto rappresentano una seria minaccia alla qualità e alla sicurezza delle cure erogate dai reparti di Medicina Interna, nonché l'espressione di un mancato allineamento fra cambiamenti demografici e organizzazione ospedaliera. E se da un lato penalizzano l'immagine delle medicine interne, dall'altro ne sanciscono la indispensabilità nel panorama ospedaliero attuale. L'aumento dei pazienti poli-patologici che trovano nelle medicine interne la loro appropriata collocazione (anche fuori reparto) rivela la necessità ormai inderogabile di passare a modelli organizzativi basati su specialisti con competenze wide-ranging, come gli internisti. La sicurezza di tali pazienti, unita all'equità e qualità delle loro cure, potrebbe rappresentare il driver di questo cambiamento.

\section{Bibliografia}

1. Stylianou N, Fackrell R, Vasilakis C, Are medical outliers associated with worse patient outcomes? A retrospective study within a regional NHS hospital using routine data. MJ Open 2017; 7:e015676.

2. Goulding L, Adamson J, Watt I, et al. Lost in hospital: a qualitative interview study that explores the perceptions of NHS inpatients who spent time on clinically inappropriate hospital wards. Health Expect 2015;18:982-94.

3. Goulding L, Adamson J, Watt I, et al. Patient safety in patients who occupy beds on clinically inappropriate wards: a qualitative interview study with NHS staff. BMJ Qual Saf 2012;21:218-24.

4. Stowell A, Claret PG, Sebbane M, et al. Hospital outlying through lack of beds and its impact on care and patient outcome. Scand J Trauma Resusc Emerg Med 2013;21:17.

5. Serafini F, Fantin G, Brugiolo R, et al. Outlier admissions of medical patients: prognostic implications of outlying patients. the experience of the hospital of Mestre. Ital J Med 2015;9:299-302.

6. Alameda C, Suárez C. Clinical outcomes in medical outliers admitted to hospital with heart failure. Eur J Intern Med 2009;20:764-7.

7. Sanderson JD, Taylor RF, Pugh S, et al. Specialized gastrointestinal units for the management of upper gastrointestinal haemorrhage. Postgrad Med J 1990;66:654-56.

8. Bucknall CE, Robertson C, Moran F, et al. Management of asthma in hospital: a prospective audit. BMJ 1988;296: 1637-9.

9. Mayor S. Stroke patients prefer care in specialist units. BMJ 2005;331:130.

10. Dunn KW. Standards and strategy for burn care. 2001 http://www. ibidb.org/downloads-mainmenu-28/cat_view /14-general-reports (accessed 14 Oct 2011). 
11. Santamaria JD, Tobin AE, Anstey MH, et al. Do outlier inpatients experience more emergency calls in hospital? an observational cohort study. Med J Aust 2014;200:45-8.

12. Lloyd JM, Elsayed S, Majeed A, et al. The practice of out-lying patients is dangerous: a multicentre comparison study of nursing care provided for trauma patients. Injury 2005;36:710-3.

13. Rae B, Busby W, Millard PH. Fast-tracking acute hospital care--from bed crisis to bed crisis. Aust Health Rev 2007;31:50-62.
14. Gilligan S, Walters M. Quality improvements in hospital flow may lead to a reduction in mortality. Clin Govern Int J 2007;13:26-34.

15. Lepage B, Robert R, Lebeau M, et al. Use of a risk analysis method to improve care management for outlying inpatients in a University hospital. Quality Safety Health Care 2009;18:441-5.

16. Creamer GL, Dahl A, Perumal D, et al. Anatomy of the ward round: the time spent in different activities. ANZ J Surg 2010;80:930-2. 


\title{
Innovazioni organizzative in Medicina Interna per la sicurezza del paziente: dall'Ambulatorio Integrato Diagnostico-Terapeutico alla Sezione di Decisione Clinica
}

\author{
Roberto Castello, ${ }^{1}$ Alberto Carli ${ }^{2}$ \\ ${ }^{1}$ Medicina Generale e SDC-AOUI di Verona; ${ }^{2}$ Scuola di Specializzazione in Igiene Medicina Preventiva, Università degli \\ Studi di Verona, Italia
}

\section{Demografia ed epidemiologia dei servizi verso la Sezione Decisione Clinica}

Assistiamo ormai da tempo in Italia ad un'evoluzione demografica che non può lasciarci indifferenti nel confrontarci con mutamenti del nostro sistema sanitario sia a livello macroscopico che nei singoli contesti operativi. Oggi l'ISTAT rileva che la popolazione anziana rappresenta il $22,6 \%$ della popolazione rispetto al 20,2\% di soli dieci anni fa, calcolando che in proiezione nel 2043 cresca fino a costituire il 32\% della popolazione. ${ }^{1}$ Tale andamento demografico, che trova ragione in un costante miglioramento degli stili di vita, delle attività di prevenzione, cura e riabilitazione offerte a tutta la popolazione, ci presenta una popolazione con una aspettativa di vita alla nascita oltre gli ottant'anni, anche per la popolazione maschile. Occorre tuttavia confrontarsi con una speranza di vita in buona salute che arriva ormai ai 58,7 anni, determinando un miglioramento costante rispetto ai 56,4 registrati nel 2009. Questi dati demografici, sicuramente confortanti, vanno letti alla luce delle caratteristiche epidemiologiche della popolazione anziana in cui si rileva un'elevata prevalenza di multi-cronicità $(49,6 \%$ degli over-sessantacinquenni). ${ }^{2}$

Il Sistema Sanitario e l'organizzazione dei servizi in questo mutante contesto epidemiologico non è rimasta una realtà statica, riorganizzando le proprie capacità di offrire servizi sanitari. Risulta evidente a

Corrispondente: Roberto Castello, Medicina Generale e SDCAOUI di Verona, Italia.

E-mail: roberto.castello@aovr.veneto.it ;

alberto.carli@aovr.veneto.it

Articolo pubblicato secondo la Creative Commons Attribution NonCommercial 4.0 License (CC BY-NC 4.0).

${ }^{\circ}$ Copyright: the Author(s), 2019

Licensee PAGEPress, Italy

QUADERNI - Italian Journal of Medicine 2019; 7(6):111-116 partire dagli anni ' 90 una progressiva deospedalizzazione delle cure mediche con una riduzione del numero di posti letto per acuti e una conseguente riduzione del tasso di ospedalizzazione in ricovero ordinario; in particolare si è passati da 123,5 ospedalizzazioni per mille residenti nel 2011 a 106,4 nel 2016. Di converso tale deospedalizzazione determina un progressivo ricorso a cure specialistiche, con una prevalenza annuale nella popolazione che sale dall' $11,9 \%$ del 2005 al 14,8\% del 2013. ${ }^{3}$ L'evoluzione del quadro epidemiologico dei servizi è stata da una parte motivata dalla presenza di vincoli di sistema con la necessità del recupero di efficienza e appropriatezza e dall'altra sostenuta da una crescente disponibilità diagnostica e terapeutica nell'ambito ambulatoriale e all'interno del cosiddetto Day Service. In tutto ciò il rischio dapprima emergente ed oggi manifesto è la frammentazione dei percorsi di cura cui si deve rispondere riconducendo all'unitarietà la gestione dell'acuzie e la presa in carico del paziente affetto da multi-cronicità e quindi definendo contesti organizzativi e strutturali adeguati a fornire cure sicure. ${ }^{4}$

Centrale in tale emergente modello di integrazione longitudinale è la presa in carico del paziente cronico, in particolare pluri-patologico con riacutizzazione, qualsiasi sia il momento del suo arruolamento in un percorso di presa in carico, ma soprattutto quando questo si rivolge al sistema sanitario per cure in urgenza in Pronto Soccorso. Fondamentale diventerà nella gestione del percorso clinico l'integrazione tra i diversi setting superando la presenza di silos assistenziali.

Il Programma Future Hospital del Royal College of Physician, nato per definire come l'ospedale debba evolversi per andare incontro al bisogno di cittadini e pazienti, ha individuato la necessità di far convergere, secondo un modello per intensità di cure, all'interno di un Acute Care Hub tutte le aree cliniche dell'ospedale che si concentrano sulla valutazione iniziale e sulla stabilizzazione dei pazienti con malattie acute tra cui l'Acute Medical Unit, il centro di assistenza ambulatoriale d'emergenza e le Short Stay Units, ponendosi come obiettivo primario il trattamento dell'acuzie 
nell'arco di 48 ore e il successivo trasferimento alle cure territoriali. ${ }^{5}$

In un'ottica di Acute Complex Care Model (ACCM) al momento del ricovero da Pronto Soccorso ci si troverà a realizzare una presa in carico globale $\mathrm{e}$ precocemente orientata alla prosecuzione del percorso di cura oltre la dimissione. I principi del modello risiedono nel fornire cure integrate attraverso la creazione di un team multi-professionale coordinato da un medico internista collocato all'interno di un ospedale strutturato per intensità di cure; risulta infatti indispensabile, per rispondere al quadro patologico emergente, inserire il ricovero del paziente in un appropriato livello di intensità di cura in un corretto bilanciamento tra rischi e benefici. ${ }^{6}$

Una possibile concretizzazione di tale modello multidisciplinare rivolto a pazienti selezionati per percorsi clinici su un orizzonte temporale definito è la Sezione di Decisione Clinica. In un ospedale per intensità di cure questa si colloca in un'area contigua al Dipartimento di Emergenza e Urgenza, dotato di Osservazione Breve Intensiva (OBI), in cui il medico di Pronto Soccorso selezioni i pazienti che rispondono a determinati Percorsi di Diagnosi e Cura (PDC). Infatti, nel progettare e realizzare una Sezione di Decisione Clinica è indispensabile individuare le sottopopolazioni patologiche di riferimento per formalizzare i rispettivi PDC, con soglie di attività che permettano una standardizzazione del processo e il razionale impiego di risorse. ${ }^{7,9}$ Per realizzare tale attività ad elevato turn-over di pazienti e consentire al tempo stesso il corretto logistic flow del percorso, è imprescindibile un appropriato impiego di tecnologie e l'estensivo utilizzo di strumenti informativi. Tra le tecnologie digitali e informatiche che possono migliorare tanto l'efficienza ospedaliera quanto la costituzione di strumenti per la sicurezza del paziente, ritroviamo la cartella clinica informatizzata e i suoi spin-off quali i software di supporto decisionale, di condivisione delle informazioni cliniche trai professionisti coinvolti anche in modo transmurale o di pianificazione del percorso post-dimissione a livello ambulatoriale o domiciliare. In particolare, l'introduzione di strumenti informatici per la prescrizione diagnostica e terapeutica quali i Computerized Physician Order Entry systems (CPOEs) ha determinato un'evoluzione nella sicurezza della gestione della terapia farmacologica; nell'ambito delle interazioni farmacologiche, un forte effetto nella riduzione dell'errore si realizza grazie al supporto decisionale. Inoltre, i CPOEs di tipo avanzato grazie all'interfaccia con la cartella clinica informatizzata consentono l'individuazione di potenziali reazioni avverse legate a quadri anafilattici o controindicazioni per co-morbidità del singolo paziente. Parimenti ad ogni altra tecnologie i CPOEs determinano nuove modalità di errore, ma offrono grazie alla tracciabilità dei dati capacità analitica strategica in un'ottica del continuo miglioramento in termini sia di sicurezza che di appropriatezza. ${ }^{9}$ Tra gli elementi caratterizzanti l'ACCM e fondamentali per lo sviluppo della cultura della sicurezza risulta il coinvolgimento del paziente e dei care-givers per sviluppare sia una alleanza costruttiva nel trattamento dell'acuzie che un adeguato proseguimento delle cure; si può ottenere un vantaggio per l'organizzazione e la sicurezza dall'engagement del paziente mutuando il suo punto di vista anche quando riportato da interviste e segnalazioni. ${ }^{10}$ Questi elementi, tipicamente parte di una visione della Patient Safety in cui sono le persone a creare la sicurezza, risultano fondamentali nel contesto dell'SDC dove per realizzare una modalità di lavoro in team multidisciplinari si dovranno sviluppare competenze non tecniche quali capacità di problem solving e leadership clinica. ${ }^{11}$

Nel contesto italiano il modello di Short Stay Unit che si concretizza nella Sezione di Decisione Clinica presenta le seguenti caratteristiche: i) attività votata all'urgenza e dedicata ai ricoveri da Pronto Soccorso; ii) arruolamento secondo Percorsi di Diagnosi e Cura (PDC) che consentano il trattamento dell'acuzie in un orizzonte temporale inferiore alle 72 ore; iii) lavoro multidisciplinare con un clinical leader internista e un team specialisti di riferimento; iv) ambiente multiprofessionale che preveda la presenza di un case manager infermieristico; v) proseguimento del percorso in postdimissione attraverso la definizione di una presa in carico longitudinale nei diversi setting assistenziali all'interno del Day Service; vi) utilizzo di un sistema informativo a sostegno del percorso integrato.

I PDC implementati, rappresentati a titolo esemplificativo in Tabella 1, devono essere aderenti alla realtà ospedaliera in cui l'SDC si inserisce e all'epi-

Tabella 1. Percorsi di diagnosi e cura (PDC).

Percorsi di diagnosi e cura

Disidratazione e disionemie

Dolore addominale non chirurgico

TIA - attacco ischemico transitorio

Trauma toracico, trauma cranico e trauma vertebrale

Anemia

Dolore toracico

Sincope

Polmonite e riacutizzazione di BPCO

Scompenso cardiaco

Scompenso glicemico

Crisi ipertensiva

Aritmie ipo e ipercinetiche

Crisi epilettica 
demiologia della popolazione ad essa afferente; tali percorsi attivati dal medico di Pronto Soccorso trovano fondamento al momento nella visita in PS nella separazione tra i diversi setting ad intensità di cure crescente quali la il rinvio al medico curante, la dimissione con presa in carico in Day Service, l'attivazione di OBI, il ricovero in SDC, il ricovero nel reparto di medicina interna o specialità cliniche, unità di alta intensità assistenziale fino alle terapie intensive. Per garantire l'appropriato utilizzo del setting assistenziale dell'SDC, che si prefigge l'obiettivo di concludere l'iter diagnostico terapeutico in 72 ore, non possono essere ricondotti all'interno dei PDC i pazienti che presentino condizioni di instabilità emodinamica, sindromi geriatriche e fragilità, preminenti bisogni specialistici, problematiche sociali o gravi patologie del comportamento. ${ }^{12}$

L'introduzione di un modello organizzativo come l'SDC non può prescindere dai principi della clinical governance (sistems for quality, evidence-based practice and policy, audit and evaluation, risk management, technology assessment, accountability and performance) ${ }^{13}$ deve prevedere l'utilizzo di un cruscotto di indicatori che consenta una valutazione di efficienza, appropriatezza dei ricoveri ed esito delle cure: i) il rispetto dei criteri di accesso definiti nei PDC; ii) la percentuale di pazienti dimessi per trasferimento in altre unità operative; iii) ricoveri ripetuti; iv) percentuale di pazienti presi in carico in Day Service.

Di concerto a quelli convenzionali per il monitoraggio dell'utilizzo di posti letto, tali indicatori consentono di dare una valutazione del percorso di cura e se associati alla misurazione dell'utilizzo di risorse diagnostiche, consulenze, etc. portano alla possibilità di valutare e misurare anche definiti PDTA dall'accesso in Pronto Soccorso alla dimissione a domicilio e follow-up ambulatoriale in post-ricovero o presa in carico in Day Service.

La valenza organizzativa, oltre che clinica, di queste misurazioni può portare alla quantificazione delle risorse impiegate e al rispettivo potenziale beneficio in merito ad una appropriata allocazione delle risorse. Da ultimo l'inclusione della misurazione di indicatori di esito, quali ri-accessi in Pronto Soccorso, ricoveri ripetuti e mortalità declinati per singolo PDC permette di ampliare l'orizzonte in un'ottica di una quantificazione del guadagno di valore realizzato sul paziente.

\section{Verso un modello di ambulatorio integrato diagnostico-terapeutico integrato}

Con la definizione e l'aggiornamento dei nuovi Livelli Essenziali di Assistenza (LEA) viene ad essere introdotto l'erogare le cure specialistiche ambulatoriali attraverso la definizione di pacchetti di prestazioni orientati ad una finalità diagnostica o terapeutica, ponendo di fatto in risalto la necessità di una presa in carico ambulatoriale coordinata. ${ }^{14}$ Il DPCM sui nuovi LEA si inserisce in un'offerta ambulatoriale cui il cittadino può accedere secondo differenti modalità, dalla richiesta di prestazioni specialistiche diagnostiche e terapeutiche o strumentali in continuità con le cure primarie, alla presa in carico di pazienti con problematiche cliniche urgenti che si affacciano al Pronto Soccorso in modo diretto fino alla presa in carico di pazienti con problematiche cliniche complesse per cui è necessaria la definizione di un percorso strutturato in ambito specialistico anche ospedaliero.

Già da tempo nell'ambito di diverse esperienze regionali sono stati definiti pacchetti ambulatoriali a partire dall'introduzione nella Regione Lazio dei Pacchetti Ambulatoriali Complessi (PAC) passando attraverso il riconoscimento di Accorpamenti di Prestazioni Ambulatoriali (APA) nel rispettivo nomenclatore tariffario. ${ }^{15} \mathrm{Si}$ introduce così in Italia il concetto di Day Service come alternativa al ricovero per pazienti che presentano bisogni sanitari complessi, individuando in un medico referente $i$ ruoli di pianificazione e coordinamento del processo assistenziale; le componenti dei singoli pacchetti sono tuttavia legate all'individuazione attraverso provvedimenti legislativi. L'istituzione del Day Service origina dal mancato riconoscimento nell'ambito del Day Hospital di tipo diagnostico di sufficienti criteri di appropriatezza organizzativa quali allettamento, necessità di assistenza medico infermieristica per più ore nel corso della giornata. A questo si aggiunge la consapevolezza che il tradizionale modello di assistenza specialistica ambulatoriale, incentrato sulle singole prestazioni e non sul problema clinico del paziente, non determina alcuna forma di presa in carico clinico-organizzativa da parte della struttura erogatrice, riconoscendo tale presa in cari l'elemento fondamentale del modello di Day Service. ${ }^{16}$ Pertanto, per garantire la deospedalizzazione delle cure ad elevato grado di coordinamento e integrazione emerge la necessità di una figura clinica di riferimento che coordini e sintetizzi i singoli bisogni diagnostico-terapeutici del paziente, dall'esecuzione di esami strumentali all'apporto dei diversi specialisti. A questa componente clinica si aggiunge una componente strutturale ed organizzativa che coordina operativamente la presa in carico complessiva rendendola erogabile sia in ambiente intra-ospedaliero che extraospedaliero. ${ }^{17}$ La gestione clinica dipende dalla capacità di sviluppare, in modo condiviso, tra i diversi specialisti che partecipano al percorso del paziente di Percorsi Ambulatoriali Complessi e Coordinati (PACC). ${ }^{8}$ Seppure diffusi in numerose realtà regionali tali pacchetti soffrono di una visione tariffaria; ad oggi manca il passaggio dal nomenclatore per prestazioni ad un sistema di remunerazione per episodio di cura/Bundle Payment. Il PAC infatti è remunerato in 
base alla somma delle tariffe delle singole prestazioni che lo hanno costituito, in presenza di limitazioni alla prescrizione di determinate prestazioni; tale limite è insito nella configurazione dei Livelli Essenziali di Assistenza, dove la costituzione di una prestazione corrisponde all'offerta di prestazione al difuori dei LEA di fatto a carico delle singole regioni con conseguenze sullo sviluppo della presa in carico. Il nuovo modello lombardo, seppure nei suoi limiti, si prefigge l'obiettivo di attuare una visione complessiva della presa in carico, spostando il punto di vista dalla prestazione erogata alla definizione di Piani Assistenziali Individuali (PAI) ed alla rispettiva gestione. ${ }^{18} \mathrm{La} \mathrm{Re}-$ gione Lombardia adotta un sistema di classificazione delle malattie croniche in categorie clinicamente significative e omogenee, cui corrisponde una modalità di remunerazione omnicomprensiva delle prestazioni necessarie per la corretta gestione dell'assistito, fatte salve eventuali forme di integrazione della tariffa prevista per il ricovero. Si include inoltre l'outcome come elemento concorrente al sistema di remunerazione con premi e penalizzazioni in funzione dell'incidenza di ricoveri e complicanze dei percorsi per la cronicità. ${ }^{19}$ Sulla base di tale sistema di classificazione si opera una stratificazione dei pazienti cronici su 3 diversi livelli di complessità per 62 patologie principali, individuando oltre alle diverse voci di spesa della presa in carico anche il possibile Gestore della stessa. ${ }^{10}$ In quest'ambito si può riconoscere il ruolo centrale di coordinamento e gestione del percorso dei pazienti, quale evoluzione del Day Service non più basato sulla erogazione di pacchetti di prestazioni ma definizione e attuazione di un piano assistenziale individuale, rappresenta un tentativo di attuazione della presa in carico secondo la teoria del valore in salute.

L'attuazione di strutture organizzative a degenza breve connesse allo sviluppo della presa in carico ambulatoriale complessa e coordinata per pazienti affetti da multi-cronicità si inserisce all'interno dell'evoluzione del ruolo dell'internista ospedaliero verso quello che può essere definito un modello di Hospitalist di tipo longitudinale, promuovendo in questo modo la sicurezza delle cure. ${ }^{20}$ Si prospetta quindi al medico internista la possibilità di seguire il percorso del paziente attraverso i diversi livelli assistenziali garantendo il coordinamento della presa in carico in una prospettiva di progressiva deospedalizzazione del trattamento precoce di patologie acute intercorrenti o riacutizzazioni nel paziente complesso e pluri-patologico.

\section{Bibliografia}

1. ISTAT. Indicatori demografici - Stime per il 2017; 2018.

2. ISTAT. Tutela della Salute e Accesso alle Cure; 2014.

3. G. Pieroni, D. Golinelli, G. Franchino. Assistenza Ospedaliera, Rapporto Osservasalute; 2017.
4. Peduzzi P. I problemi di salute emergenti e i riflessi sull'offerta sanitaria. Gruppo di Lavoro Primary Health Care della Società Italiana di Igiene Medicina Preventiva e Sanità Pubblica. Governare l'Assistenza Primaria, Manuale per Operatori di Sanità Pubblica. Milano: Bruno Mondadori - Ricerca; 2016. pp 31-2.

5. Royal College of Physicians. Future Hospital: Caring for medical patient; 2013.

6. Pietrantonio F, Orlandini F, Moriconi L, La Regina M. Acute Complex Care Model: An Organizational approach for the medical care of hospitalized acute complex patients. Eur J Intern Med 2015;26:759-65.

7. Vincent C. Interventi clinici e miglioramento del processo; Progettazione, tecnologia e standardizzazione. La sicurezza del paziente. Milano: Springer-Verlag Italia; 2011. pp 213-14.

8. Powter L, Beale A, Probert B, Dhanda A. Development and validation of a tool to select patients for admission to medical short stay units. Clin Med 2014;14:371-5.

9. Schiff GD, Amato MG, Eguale T, et al. Computerised physician order entry-related medication errors: analysis of reported errors and vulnerability testing of current systems BMJ Qual Saf 2015;24:264-71.

10. Gallagher TH, Mazor KM. Taking complaints seriously: using the patient safety lens. BMJ Qual Saf 2015;24:352-5.

11. Royal College of Physicians. Acute medical care. The right person, in the right setting - first time. 2007:22-4.

12. Juan A, Salazar A, Alvarez A, et al. Effectiveness and safety of an emergency department short stay unit as an alternative to standard inpatient hospitalization. Emerg Med J 2006;23:833-7.

13. Ministero della Salute. Il Governo Clinico: una prospettiva sistemica; 2007.

14. Decreto del Presidente del Consiglio dei Ministri del 12 gennaio 2017 "Definizione e aggiornamento dei livelli essenziali di assistenza, di cui all'articolo 1, comma 7, del decreto legislativo 30 dicembre 1992, n. 502. (17A02015). C. 5, Art. 15 “Assistenza Specialistica Ambulatoriale".

15. Regione Lazio, Deliberazione della Giunta Regionale 4 agosto 2005 n. 731 "Ripartizione nei livelli di assistenza del fondo sanitario regionale 2005. Finanziamento del livello assistenziale ospedaliero per l'anno e definizione del sistema di remunerazione delle prestazioni ospedaliere dei soggetti erogatori pubblici e privati per l'anno 2005. Finanziamento e definizione del sistema di remunerazione delle prestazioni di assistenza specialistica ambulatoriale e delle attività di assistenza riabilitativa territoriale".

16. Ministero della Salute. Progetto Mattoni SSN; Evoluzione del sistema DRG nazionale - Milestone 1.2.1. Forme alternative alla degenza: definizione (day hospital, day surgery, day service) e relativi flussi informativi. Relazione finale approvata il 16 maggio 2007.

17. Regione Lazio, Decreto del Commissario ad Acta 19 maggio 2014 n. 100155 "Criteri per l'erogazione dei Pacchetti Ambulatoriali Complessi (PAC) in strutture ambulatoriali extraospedaliere. Introduzione di ulteriori Pacchetti Ambulatoriali Complessi (PAC) sia in strutture ospedaliere che extraospedaliere.

18. Regione Lombardia, Delibera Giunta Regionale 30 gennaio 2017 n.6164 "Governo della domanda: avvio 
della presa in carico di pazienti cronici e fragili. Determinazioni in attuazione dell'art. 9 della legge $n$. 23/2015".

19. Regione Lombardia, Legge Regionale 11 agosto 2015 n. 23 "Evoluzione del sistema sociosanitario lombardo: modifiche al Titolo I e al Titolo II della legge regionale
30 dicembre 2009, n. 33 (Testo unico delle leggi regionali in materia di sanità)". Art. 9 "Modelli di presa in carico per il paziente cronico e fragile".

20. Orlandini F, La Regina M, Verdiani V, Manfellotto D. La nuova figura professionale in Italia: profilo, attività e modelli organizzativi. Ital J Med 2018;37-42. 


\section{Ossigenoterapia}

Micaela La Regina

SS Risk Management, ASL 5 Liguria, La Spezia, Italia

Corrispondente: Micaela La Regina, S.S. Risk Management, ASL 5 Spezzino, via Fazio 30, 19121 La Spezia, Italia.

Tel.: +39.0187533782.

E-mail: micaela.laregina@asl5.liguria.it

Articolo pubblicato secondo la Creative Commons Attribution NonCommercial 4.0 License (CC BY-NC 4.0).

${ }^{\circ}$ Copyright: the Author(s), 2019

Licensee PAGEPress, Italy

QUADERNI - Italian Journal of Medicine 2019; 7(6):117-118
L'ossigeno è a tutti gli effetti un farmaco e, per di più, il farmaco più prescritto in ambito ospedaliero. Può essere utilizzato in tutte le specialità e la sua gestione coinvolge varie tipologie di operatori sanitari. L'ossigeno è indicato in molte condizioni critiche, ed è un farmaco salvavita, in quanto previene l'ipossiemia severa. Tuttavia potenzialmente può causare seri danni o anche la morte, se non è somministrato e gestito in maniera appropriata.

La National Patient Safety Agency (NPSA) ha pubblicato nel 2009 un resoconto di 281 incident reporting in cui un'inappropriata prescrizione e gestione dell'ossigeno ha causato 9 decessi e contribuito ad altri $35 .^{1}$

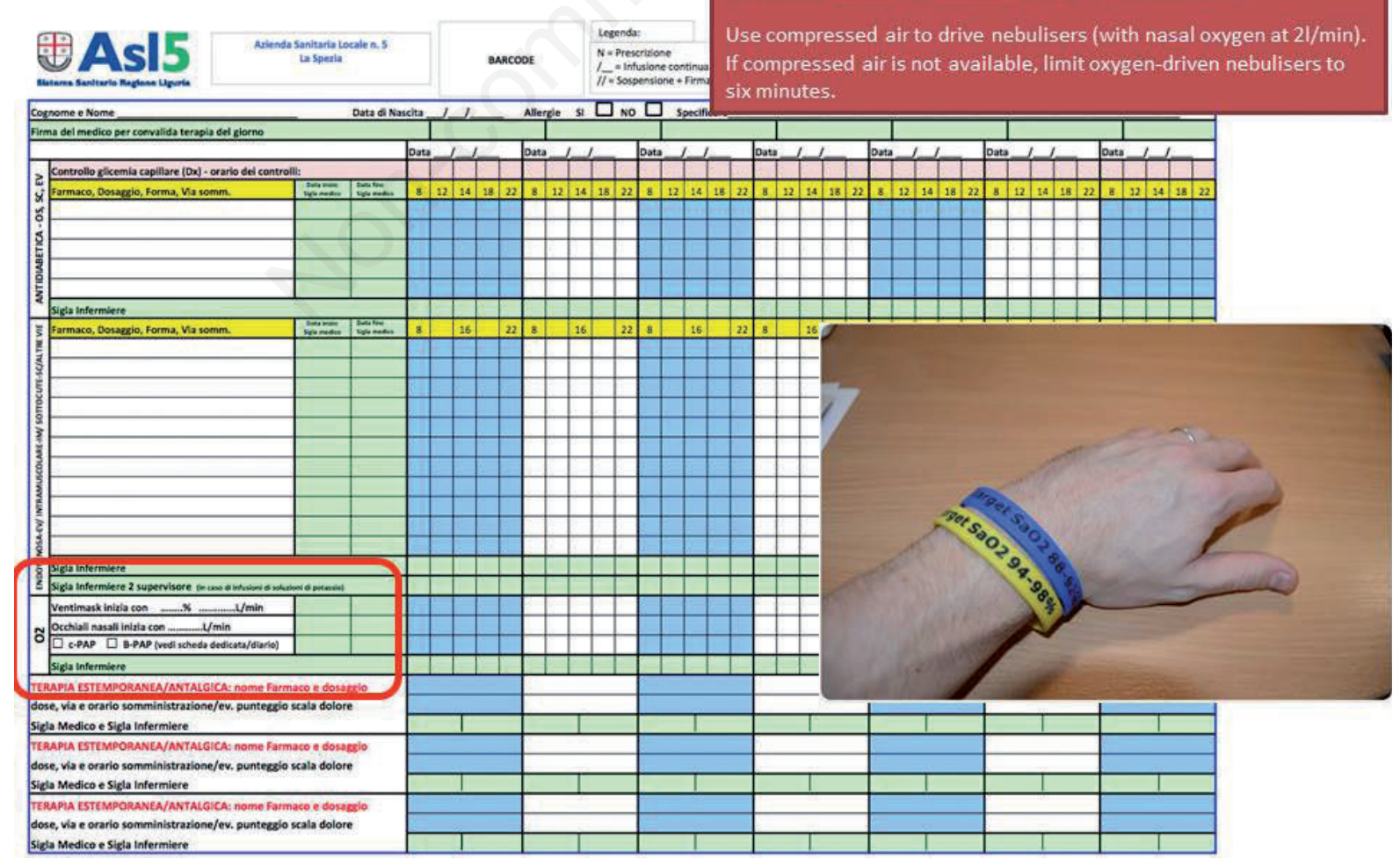

Figura 1. Esempi di strategie per migliorare la sicurezza dell'ossigenoterapia. 
L'analisi di questi eventi ha evidenziato varie modalità di errore in diversi step del processo di gestione dell'ossigenoterapia: i) prescrizione: mancata o errata prescrizione; somministrazione di $\mathrm{O}_{2}$ senza prescrizione scritta; ii) monitoraggio: mancato monitoraggio o mancato intervento in caso di livelli di saturazione di $\mathrm{O}_{2}$ alterati; iii) somministrazione: confusione fra ossigeno e aria compressa o altri gas, flussi errati, disconnessione inavvertita del flusso; iv) apparecchiature: bombole vuote, apparecchiature mancanti.

Ha pertanto emanato le seguenti raccomandazioni per migliorare la sicurezza dell'ossigenoterapia: i) chiediti sempre se il paziente necessita di ossigeno. L'uso routinario di ossigeno nei pazienti con infarto miocardico, stroke o dispnea senza insufficienza respiratoria non è supportato dalle evidenze; ii) prescrivi l'ossigenoterapia indicando il target di saturazione periferica $\left(\mathrm{SpO}_{2}\right)$ da raggiungere: $94-98 \%$ per i pazienti critici ipossiemici e $88-92 \%$ nei pazienti a rischio di ipercapnia (obesi, cifoscoliotici e affetti da altre sindromi restrittive, pazienti con malattie neuromuscolari) o con insufficienza respiratoria ipercapnica manifesta; iii) utilizza il dispositivo di somministrazione appropriato. Le cannule nasali sono adeguate per la maggior parte dei pazienti; la maschera con reservoir deve essere riservata a casi limitati di pazienti critici. Utilizza una ventimask al $28 \%$ per i pazienti ad alto rischio con BPCO o che necessitano di ossigeno a basse dosi; iv) riporta sempre nella scheda di terapia il target, il dispositivo di erogazione, la dose (flusso e $\mathrm{fiO}_{2} \%$ ), come indicato in Figura 1. Registra in cartella clinica i risultati dell'emogasanalisi di controllo in corso di ossigenoterapia; v) controlla i gas ematici me- diante emogasanalisi in tutti i pazienti critici e, in particolare, se sospetti acidosi o ipercapnia. La saturimetria periferica non dà informazioni su $\mathrm{pH}$ e $\mathrm{pCO}_{2} ; \mathrm{vi}$ ) monitorizza i pazienti in ossigenoterapia utilizzando dei sistemi per l'identificazione precoce del deterioramento clinico (es. NEWS); vii) in caso di emergenza, non ritardare la somministrazione dell'ossigeno, per effettuare la prescrizione scritta.

Non va trascurata neanche l'educazione di pazienti e caregiver e del personale di supporto (operatori socio-sanitari) al corretto utilizzo della terapia con ossigeno.

Una recente revisione sistematica di tutti gli studi che hanno valutato la prescrizione e somministrazione dell'ossigeno, nonché gli interventi per migliorare la sicurezza dell'ossigenoterapia come linee guida aziendali, sessioni educative, alert stickers, schede di prescrizione dedicata, ecc., ha evidenziato numerose carenze nella prescrizione e somministrazione, l'efficacia, ma difficile sostenibilità di alcune azioni di miglioramento e la necessità di indagare fattori facilitatori e barriere per migliorare in maniera permanente la pratica clinica e la sicurezza dei pazienti. ${ }^{2}$

\section{Bibliografia}

1. Lamont T, Luettel D, Scarpello J. Improving the safety of oxygen therapy in hospitals: summary of a safety report from the National Patient Safety Agency. BMJ 2010;340:c187.

2. Cousins JL, Wark PAB, McDonald VM. Acute oxygen therapy: a review of prescribing and delivery practices. Int J Chron Obstruct Pulmon Dis 2016;11:1067-75. 


\title{
Ventiloterapia
}

\author{
Micaela La Regina \\ SS Risk Management, ASL 5 Liguria, La Spezia, Italia
}

La ventilazione meccanica non invasiva, grazie al suo potenziale di impiego al di fuori della terapia intensiva, ad esempio in medicina interna, ha dimostrato di poter ridurre significativamente la mortalità, il ricorso all'intubazione e alla ventilazione meccanica soprattutto nei pazienti con riacutizzazione di BPCO.

Una revisione $^{1}$ degli eventi avversi in corso di ventilazione non invasiva ha evidenziato un aumento dei rischi quando: i) i pazienti, soprattutto quelli incapaci di chiedere aiuto, non sono adeguatamente monitorizzati; ii) gli allarmi vengono disattivati dallo staff; iii) lo staff non ha familiarità con i ventilatori e il loro corretto uso (per esempio se richiedono valvola per $\mathrm{CO}_{2}$ o no; in caso di pazienti che portano in ospedale gli apparecchi domiciliari); iv) viene implementato un nuovo ventilatore o una nuova interfaccia senza formazione.

Al fine di migliorare la sicurezza della ventiloterapia non invasiva, la Joint Commission International raccomanda una serie di azioni: ${ }^{2,3}$

- a breve termine: i) dotarsi di una istruzione operativa per la ventiloterapia, aggiornata e condivisa con lo staff; ii) elaborare una checklist per ogni modello di ventilatore in uso nel reparto e assicurarsi che ogni membro dello staff conosca la gestione di ciascun modello (in particolare, assemblaggio del circuito, definizione dei controlli e degli allarmi); iii) documentare training e periodico re-training dello staff.

- e a lungo termine: i) verificare annualmente le competenze dello staff; ii) mettere a disposizione il materiale utilizzato per la formazione; iii) creare un team multidisciplinare con ruoli e criteri chiari per l'intervento delle varie figure (es. ricorso all'intubazione e trasferimento in terapia intensiva). Le linee guida della British Thoracic Society raccomandano come buone pratiche: ${ }^{4}$ i) il monitoraggio continuo della saturazione periferica di $\mathrm{O}_{2}$; ii) controlli intermittenti del $\mathrm{pH}$ e della $\mathrm{pCO}_{2}$ mediante emogasanalisi: iii) monitoraggio elettrocardiografico continuo in caso di frequenza cardiaca $>120 /$ min o aritmie o possibili cardiopatie associate.

\section{Bibliografia}

1. https://www.england.nhs.uk/wp-content/uploads/ 2015/02/psa-niv.pdf

2. Safety in respiratory care: a guide to JCAHO requirements. Available from: http://hcpro.com/content/ 32186.pdf

3. Bhimji SS, Sharma S. Ventilator safety. StatPearls; 2018.

4. BTS Guidelines for ventilatory management of AHRF; March 2016.
Corrispondente: Micaela La Regina, S.S. Risk Management, ASL 5 Spezzino, via Fazio 30, 19121 La Spezia, Italia. Tel.: +39.0187533782 .

E-mail: micaela.laregina@as15.liguria.it

Articolo pubblicato secondo la Creative Commons Attribution NonCommercial 4.0 License (CC BY-NC 4.0).

${ }^{\circ}$ Copyright: the Author(s), 2019

Licensee PAGEPress, Italy

QUADERNI - Italian Journal of Medicine 2019; 7(6):119-120 


\title{
Strumenti per una gestione sicura delle procedure invasive
}

\author{
Micaela La Regina \\ SS Risk Management, ASL 5 Liguria, La Spezia, Italia
}

Corrispondente: Micaela La Regina, S.S. Risk Management, ASL 5 Spezzino, via Fazio 30, 19121 La Spezia, Italia. Tel.: +39.0187533782 .

E-mail: micaela.laregina@asl5.liguria.it

Articolo pubblicato secondo la Creative Commons Attribution NonCommercial 4.0 License (CC BY-NC 4.0).

${ }^{\circ}$ Copyright: the Author(s), 2019

Licensee PAGEPress, Italy

QUADERNI - Italian Journal of Medicine 2019; 7(6):121-122

Si considera procedura invasiva ${ }^{1}$ qualunque manovra diagnostica o terapeutica, effettuata in sala operatoria o al di fuori di essa, che preveda: i) l'esecuzione di un taglio o un foro per accedere all'interno del corpo di un paziente - ad esempio, quando si esegue un'operazione o si inserisce catetere vascolare o si pratica una biopsia; ii) l'accesso a una cavità corporea (come il sistema digestivo, i polmoni, l'utero o la vescica) senza incisioni; iii) l'utilizzo di radiazioni elettromagnetiche (raggi x, laser, raggi gamma, luce ultravioletta), ad esempio le procedure di cardiologia interventistica.

Le procedure che più frequentemente vengono effettuate nei reparti di medicina interna autonomamente dagli internisti sono: toracentesi, paracentesi, rachicentesi, biopsia osteo-midollare, posizionamento di accessi venosi centrali.

Recentemente la National Patient Safety Agency (NPSA) ha emanato delle raccomandazioni per l'esecuzione in sicurezza degli interventi chirurgici e di tutte le procedure invasive, scaturite dall'analisi degli eventi avversi raccolti nel Regno Unito. Tali raccomandazioni sono distinte in due gruppi (Tabella 1). Le prime 5 sono di tipo organizzativo, le altre otto sono di tipo operativo e vengono definite nel documento sequenziali perché rappresentano una sequenza logica di passaggi che devono essere eseguiti per ogni procedura e per ogni paziente. Tali passaggi possono essere inclusi in una checklist dedicata per ogni tipo di procedura. La NPSA enfatizza molto la necessità di standardizzare, armonizzare e istruire.

A titolo di esempio, un recente lavoro sullo stato dell'arte della sicurezza della toracentesi ${ }^{3}$ riconosce come fattori in grado di migliorare la sicurezza: la guida ecografica, l'uso del triangolo di sicurezza (delimitato anteriormente dal margine laterale del pettorale maggiore, lateralmente dal margine laterale del muscolo latissimo del dorso, superiormente dalla base dell'ascella e inferiormente dal $\mathrm{V}$ spazio intercostale), l'uso di una checklist (Tabella 2), la valutazione delle competenze degli operatori, la formazione mediante simulazione e la creazione di team dedicati.

Tabella 1. Standard di sicurezza per le procedure invasive (NatSSIPs).

\begin{tabular}{ll}
\hline Standard organizzativi & Standard operativi \\
\hline $\begin{array}{l}\text { Governance e audit: } \\
\text { - sistema di segnalazione e analisi di eventi e quasi eventi }\end{array}$ & $\begin{array}{l}\text { Verifica della procedura da eseguire, } \\
\text { del paziente, della sede e del lato }\end{array}$ \\
- audit sulla compliance alle presenti raccomandazioni e diffusione dei risultati & \\
- creazione di team per ogni procedura con un responsabile della governance & Briefing di sicurezza \\
\hline $\begin{array}{l}\text { Condivisione delle esperienze fra le organizzazioni } \\
\text { pre-procedura/ anestesia o sedazione/descrizione procedura/trattamento post-procedura }\end{array}$ & Sign-in \\
\hline $\begin{array}{ll}\text { Forza lavoro: numero adeguato di operatori adeguatamente formati } \\
\text { Prenotazione e gestione della lista: standardizzazione del data-set e del linguaggio usato } \\
\text { (abbreviazioni permesse), criteri per la gestione della lista, gestione del cambio di lista }\end{array}$ & Time-out \\
\hline $\begin{array}{l}\text { Trasferimento delle informazioni: passaggio di consegne strutturato, senza interruzioni e, } \\
\text { possibilmente con la partecipazione del paziente }\end{array}$ & $\begin{array}{l}\text { Verifica della protesi } \\
\text { Prevenzione della ritenzione di corpi estranei }\end{array}$ \\
& $\begin{array}{l}\text { Sign-out } \\
\text { Debriefing }\end{array}$ \\
\hline
\end{tabular}


Tabella 2. Modello di checklist per la toracentesi o il posizionamento di drenaggio pleurico - Un membro del team legge la checklist e gli altri confermano che ciascuna delle azioni è stata effettuata.

\section{BRIEFING}

Diagnosi e indicazione alla procedura

Comorbidità rilevanti

Storia clinica e obiettività recenti

Sospensione anticoagulanti, se in atto

Allergie (ad anestetici)

Esami di laboratorio

PLT $>100.000 / \mathrm{mmc}$

PT-INR

aPTT

Visione Rx torace

Controllo materiale (guanti sterili, kit, anestetici, disinfettanti, provette campioni)

Disponibilità ecografo

\section{SIGN-IN}

Identificazione del paziente e della procedura

Spiegazione della procedura al paziente

Consenso informato firmato

Allergie

Parametri vitali pre-procedura

Posizionamento del paziente

Identificazione ecoguidata del punto di inserzione

Marcatura del punto di inserzione

Lavaggio mani

Creazione campo sterile

Disinfezione

TIME-OUT

Immediatamente prima di iniziare la procedura:

Controllo identità del paziente

Nome procedura da eseguire

Sito di inserzione

Allergie

\section{SIGN-OUT}

Nome della procedura eseguita, sede e lato

Conta strumenti utilizzati

Conferma campioni da inviare

Verifica correttezza dell'etichettatura dei campioni e della richiesta

Indicazioni post-procedura (es. frequenza monitoraggio parametri vitali, emogasanalisi e richiesta RX torace postprocedura)

Descrizione della procedura in cartella, secondo schema standardizzato

\section{DEBRIEFING}

Cosa è andato bene

Cosa è andato male

Eventuali azioni di miglioramento

\section{Bibliografia}

1. Sevdalis N. Safety standards for invasive procedures. BMJ 2016;352i1121.

2. National Safety Standards for Invasive Procedures
(NatSSIPS). Available from: https://improvement.nhs. uk/documents/923/natssips-safety-standards.pdf

3. De Biasi EM, Puchalski J. Thoracentesis: state-of-theart in procedural safety, patient outcomes, and physiologic impact. Pleura 2016;3:1-10. 


\title{
La prevenzione del tromboembolismo venoso
}

\author{
Fulvio Pomero, ${ }^{1}$ Elena Nicola, ${ }^{1}$ Pietro Salomone, ${ }^{2}$ Roberta Risso ${ }^{1}$ \\ ${ }^{1}$ Dipartimento di Medicina Interna, ASL CN2, Alba-Bra $(\mathrm{CN}) ;{ }^{2}$ Scuola di Specialità in Medicina Interna, Università degli \\ Studi di Torino, Italia
}

\section{Introduzione}

L'ospedalizzazione per patologia medica acuta determina un incremento del rischio trombotico di circa 8 volte ed è responsabile di circa il $25 \%$ di tutti gli eventi tromboembolici. ${ }^{1}$ In ambito ospedaliero più della metà degli episodi di TEV insorgono in Dipartimenti Medici. La stratificazione del rischio trombotico di pazienti medici ospedalizzati risulta spesso complessa a causa della loro eterogeneità. ${ }^{2}$

Al fine di identificare i pazienti ad elevato rischio di TEV che occorre sottoporre a tromboprofilassi è stato derivato e validato uno score di rischio (Padua Prediction Score) che prende in considerazione 11 fattori di rischio trombotico e classifica i pazienti in basso rischio ( $<4$ punti; circa $60 \%$ dei pazienti) o alto rischio ( $\geq 4$ punti; circa $40 \%$ dei pazienti) in base al punteggio totalizzato. Durante il follow-up di 3 mesi l'incidenza di TEV sintomatico in assenza di profilassi nel gruppo a basso rischio era dello $0.3 \%$ mentre risultava dell' $11 \%$ nei pazienti ad alto rischio (rischio relativo $=32) .{ }^{3}$ In considerazione di questi dati e della necessità di uniformare la stratificazione del rischio in ambiente medico il Padua Prediction Score è stato raccomandato dalla IX edizione delle linee guida ACCP come strumento per la definizione del paziente ad aumentato rischio trombotico da sottoporre a tromboprofilassi $^{2}$ (Tabella 1).

Sebbene il rischio emorragico attribuito alla tromboprofilassi farmacologica non sia particolarmente elevato è necessario stratificare il rischio individuale del paziente prima di procedere alla prescrizione al

Corrispondente: Fulvio Pomero, U.O. Medicina Interna, Ospedale S. Lazzaro, via P. Belli 26, 12100 Alba (CN), Italia. Tel.: +39.0173316863.

E-mail: fulviopomero@yahoo.it

Articolo pubblicato secondo la Creative Commons Attribution NonCommercial 4.0 License (CC BY-NC 4.0).

${ }^{\circ}$ Copyright: the Author(s), 2019

Licensee PAGEPress, Italy

QUADERNI - Italian Journal of Medicine 2019; 7(6):123-126 fine di ridurre il rischio clinico di questa misura farmacologica. Un recente studio osservazionale multicentrico ha valutato $\mathrm{i}$ fattori di rischio di sanguinamento su 10.866 pazienti medici ospedalizzati. ${ }^{4}$ L'ulcera gastrointestinale attiva, un episodio di sanguinamento negli ultimi 3 mesi ed una conta piastrinica $<50.000 / \mathrm{mm}^{3}$ sono risultati i fattori di rischio maggiormente pericolosi seguiti dall'età maggiore di 85 anni, l'insufficienza epatica, l'insufficienza renale severa (filtrato $<30 \mathrm{~mL} / \mathrm{min}$ ) ed il ricovero in terapia intensiva. Sulla base della forza di associazione tra questi fattori di rischio e gli episodi di sanguinamento è stato generato uno score che ha permesso di evidenziare che più della metà degli episodi di sanguinamento maggiore avveniva nel $10 \%$ dei pazienti che possedeva un valore $\geq 7$ (Tabella 2 ). ${ }^{4}$

È stato dimostrato che la profilassi farmacologica riduce significativamente l'insorgenza di embolia polmonare sintomatica (RR 0.43) e di embolia polmonare fatale (RR 0.38) senza incrementare significativamente il rischio di sanguinamento maggiore (RR 1.32). ${ }^{5}$

Le evidenze disponibili sulla tromboprofilassi farmacologica dimostrano un'efficacia simile tra l'eparina a basso peso molecolare (LMWH) e l'eparina non frazionata (LDUH) nel ridurre la trombosi in pazienti medici ospedalizzati con una lieve tendenza al minor sanguinamento per la LMWH. L'efficacia del fondaparinux è sovrapponibile a quella dell'eparina nella riduzione degli eventi trombotici e nel rischio di sanguinamento. ${ }^{2}$

Nei pazienti con rischio emorragico aumentato occorre prendere in considerazione l'uso di metodi meccanici di profilassi come la compressione elastica (GCS), la compressione pneumatica intermittente (IPC) e la pompa plantare (VFP) che riducono la stasi ematica inducendo un passaggio di sangue dal distretto venoso superficiale a quello profondo attraverso le vene perforanti. ${ }^{2}$

I rischi conseguenti alla somministrazione delle LMWH e del fondaparinux in alcune popolazioni (paziente anziano, obeso, sottopeso o con insufficienza renale) sono particolarmente elavati e rendono indispensabili alcune considerazioni specifiche.

Dati italiani provenienti dallo studio Gemini riguardante una popolazione ricoverata in Medicina In- 
terna mostrano come il $48 \%$ dei pazienti abbia un'età maggiore di 75 anni ( $6 \%>90$ anni), il 18,7\% presenti un BMI $>30$, il 5,6\% un BMI $<18,5$; inoltre circa il $40 \%$ dei ricoverati possiede una clearance creatininica $<50 \mathrm{~mL} / \mathrm{min}$ ( $15 \%$ filtrato glomerulare $<30 \mathrm{~mL} / \mathrm{min}){ }^{6}$

\section{Paziente anziano}

Non è chiaro quale influenza abbia l'età sulla cinetica di questi farmaci, ma è certo che il paziente anziano possiede un rischio emorragico incrementato rispetto alla popolazione generale. In effetti in un recente studio è emerso che il rischio di emorragia maggiore in pazienti ultraottantenni trattati con anticoagulanti è 2,5 volte più elevato rispetto ad una popolazione di età inferiore a 80 anni e il rischio relativo di emorragia intracranica è 4,2. D'altro canto un paziente di circa 80 anni possiede un rischio di TEV circa 10 volte superiore rispetto ad un paziente di 30 anni. E stato dimostrato che l'utilizzo di enoxaparina in pazienti ricoverati in Medicina Interna riduce il rischio di TEV del $63 \%$ nell'intera popolazione mentre il rischio scendeva dell' $87 \%$ quando venivano analizzati solamente i pazienti con età maggiore di 80 anni. Le linee guida ACCP ci aiutano a gestire il risk management identificando l'età avanzata come determinante per un'attenta valutazione della funzione renale. $^{2}$

\section{Paziente obeso}

L'obesità ed il sovrappeso sono importanti fattori di rischio acquisito per TEV (rischio relativo 2-3). L'interrogativo riguardo l'utilizzo di LMWH e fondaparinux nell'obeso è: i dosaggi abituali di LMWH sono adeguati oppure è necessario aumentarli?

Il paziente obeso ha un basso rapporto tra massa magra, intensamente vascolarizzata, e massa grassa con scarsa presenza di vasi sanguigni; il risultato di questa condizione potrebbe essere quello di ottenere elevate concentrazioni plasmatiche utilizzando un dosaggio basato sul peso corporeo totale. D'altra parte l'elevato rischio trombotico del paziente obeso non consente di ridurre empiricamente i dosaggi perché si potrebbero ottenere concentrazioni sub-terapeutiche che conducono a fallimenti clinici.

L'utilizzo di enoxaparina alla dose di 0,5 $\mathrm{mg} / \mathrm{kg} /$ die in pazienti obesi ricoverati in Medicina Interna ottenendo valori di attività anti-Xa a 4-6 h compresi nel range della profilassi $(0,2-0,6) .^{7}$ Questi dati suggeriscono pertanto che dosaggi di enoxaparina basati sul peso corporeo e pertanto aumentati rispetto a quelli consueti, siano più efficaci rispetto ai classici dosaggi standard.

In parziale contrasto con questi dati è lo studio eseguito con parnaparina in pazienti obesi sottoposti a chirurgia bariatrica dove la dose usuale di parnaparina (4250 UI/die) otteneva un picco di attività anti-Xa in range profilattico $(0,1-0,4)$ nel $98,3 \%$ dei casi, mentre dosaggi più elevati $(6400 \mathrm{UI} /$ die) determinavano valori eccessivi $(>0,4)$ nel $64,3 \%$ dei casi. ${ }^{8}$ È possibile che le differenze tra le diverse LMWH possano essere in relazione alla variabilità con la quale esse sono capaci di penetrare nel tessuto adiposo.

In sintesi di fronte al paziente con obesità importante dobbiamo ricordare che esistono differenze tra le diverse LMWH e scegliere accuratamente il dosaggio profilattico in base al principio attivo utilizzato.

Tabella 1. Score di rischio trombotico nel paziente medico ospedalizzato (Padua Prediction Score).

\begin{tabular}{lc}
\hline Caratteristiche del paziente & Punteggio \\
\hline Cancro & 3 \\
\hline Precedente TEV & 3 \\
\hline Ridotta mobilità & 3 \\
\hline Trombofilia nota & 3 \\
\hline Recente trauma o intervento chirurgico $(<30 \mathrm{gg})$ & 2 \\
\hline Età $\geq 70$ anni & 1 \\
\hline Insufficienza respiratoria o cardiaca & 1 \\
\hline Stroke ischemico o infarto miocardico & 1 \\
\hline Patologie reumatologiche o infettive acute & 1 \\
\hline Obesità & 1 \\
\hline Terapia ormonale & 1 \\
\hline
\end{tabular}

Score: $<4$ basso rischio; $\geq 4$ rischio elevato.

Tabella 2. Score di rischio emorragico nel paziente medico ospedalizzato (IMPROVE).

\begin{tabular}{lc}
\hline Fattori di rischio & Punti \\
\hline Insufficienza renale moderata (GFR $30-59 v s \geq 60 \mathrm{~mL} / \mathrm{min})$ & 1 \\
\hline Maschi $v s$ femmine & 1 \\
\hline Età $40-84$ aa $v s<40$ aa & 1.5 \\
\hline Cancro attivo & 2 \\
\hline Malattie reumatiche & 2 \\
\hline Catetere venoso centrale & 2 \\
\hline ICU/CCU & 2.5 \\
\hline Insufficienza renale severa $(\mathrm{GFR}<30 v s \geq 60 \mathrm{~mL} / \mathrm{min})$ & 2.5 \\
\hline Insufficienza epatica $(\mathrm{INR}>1.5)$ & 2.5 \\
\hline Età $\geq 85$ aa $v s<40$ aa & 3.5 \\
\hline Conta piastrinica $<50 \times 10^{9}$ cell $/ \mathrm{L}$ & 4 \\
\hline Sanguinamento nei 3 mesi precedenti & 4 \\
\hline Ulcera gastroduodenale attiva & 4.5 \\
\hline
\end{tabular}




\section{Paziente con insufficienza renale}

Sia le LMWH che il fondaparinux vengono eliminati per via renale per cui, in condizione di alterata funzionalità renale, risulta ridotta la clearance del farmaco e aumenta la sua emivita.

È noto che la corretta valutazione della funzione renale può infatti essere validamente effettuata con il calcolo della clearance della creatinina attraverso la formula di Cockroft-Gault o con l'MDRD ma deve essere ricordato che questi algoritmi non risultano intercambiabili e danno risultati non similari.

La valutazione del picco di attività anti-Xa dopo somministrazione di enoxaparina a dosaggio profilattico in pazienti con vario grado di insufficienza renale ha dimostrato che, in caso di insufficienza renale severa (filtrato glomerulare inferiore a $30 \mathrm{~mL} / \mathrm{min}$ ), l'attività anti-Xa risulta significativamente incrementata sia al primo che al quarto giorno della somministrazione. ${ }^{9}$

I dati provenienti da uno studio eseguito su pazienti critici dimostrano che la dalteparina, quando somministrata alla dose di $5000 \mathrm{UI} /$ die, non determina fenomeni di accumulo anche in presenza di insufficienza renale grave. La popolazione in esame era trattata frequentemente con amine che attraverso la vasocostrizione periferica avrebbero potuto condizionare un ridotto assorbimento. Successivamente un nuovo lavoro ha concluso che la dalteparina alle dosi comunemente indicate per la tromboprofilassi non determina un bioaccumulo maggiore del $30 \%$ in pazienti con insufficienza renale severa trattati per 10 giorni. ${ }^{10}$

Il fondaparinux è un pentasaccaride di circa 1700 dalton e possiede un'emivita di circa 17 ore. L'insufficienza renale determina un decremento della clearance del farmaco e in particolare essa viene ridotta al $50 \%$ con filtrato compreso tra 30 e $50 \mathrm{~mL} / \mathrm{min}$ e al $20 \%$ con filtrato inferiore a $30 \mathrm{~mL} / \mathrm{min}$. L'emivita del farmaco in presenza di insufficienza renale severa raggiunge le 72 ore. Per queste ragioni nel paziente con funzione renale gravemente compromessa i dosaggi profilattici di fondaparinux devono essere ridotti. In particolare se la clearance della creatinina è compresa tra 20 e $50 \mathrm{~mL} / \mathrm{min}$ il dosaggio raccomandato è 1,5 $\mathrm{mg} /$ die mentre in presenza di clearance della creatinina inferiore a $20 \mathrm{~mL} / \mathrm{min}$ il fondaparinux non è raccomandato. ${ }^{11}$

L'utilizzo dell'eparina non frazionata (es. eparina calcica 5000 UI tid) è da considerare un valido approccio per la tromboprofilassi nel paziente con grave compromissione della funzione renale. ${ }^{2}$

\section{Conclusioni}

La profilassi del TEV in Medicina Interna è una pratica essenziale e deve tenere conto del rischio trombotico ed emorragico che oggi è possibile stratificare agevolmente con il Padua Prediction Score e con l'Improve score.

Dopo aver correttamente identificato il paziente da trattare occorre scegliere accuratamente la molecola ed il dosaggio opportuno ricordando che le caratteristiche individuali del soggetto possiedono una fondamentale importanza nell'orientare le nostre azioni.

\section{Bibliografia}

1. Heit JA, Silverstein MD, Mohr D et al. Risk factors for deep vein thrombosis and pulmonary embolism: a population-based case-control study. Arch Intern Med 2000;160:809-15.

2. Kahn SR, Lim W, Dunn AS, et al. Prevention of VTE in Nonsurgical Patients Antithrombotic Therapy and Prevention of Thrombosis, 9th ed: American College of Chest Physicians. Evidence-Based Clinical Practice Guidelines. Chest 2012;141:e195S-e226S.

3. Barbar S, Noventa F, Rossetto V, et al. A risk assessment model for the identification of hospitalized medical patients at risk for venous thromboembolism: the Padua Prediction Score. J Thromb Haemost 2010;8:2450-7.

4. Decousus H, Tapson VF, Bergmann JF, et al. Factors at admission associated with bleeding risk in medical patients: findings from the IMPROVE investigators. Chest 2011;139:69-79.

5. Dentali F, Douketis JD, Gianni M, et al. Meta-analysis: anticoagulant prophylaxis to prevent symptomatic venous thromboembolism in hospitalized medical patients. Ann Intern Med 2007;146:278-88.

6. Gussoni G, Campanini M, Silingardi M, et al. In-hospital symptomatic venous thromboembolism and antithrombotic prophylaxis in Internal Medicine. Thromb Haemost 2009; 101:893-901.

7. Rondina MT, Wheeler M, Rodger M, et al. Weightbased dosing of enoxaparin for VTE prophylaxis in morbidly obese, medically-Ill patients. Thromb Res 2010;125:220-3.

8. Imberti D, Legnani C, Baldini E, et al. Pharmacodynamics of low molecular weight heparin in patients undergoing bariatric surgery: a prospective, randomised study comparing two doses of parnaparin. Thromb Res 2009;124:667-71.

9. Sanderink CM, Guimart CG, Ozoux ML, et al. Pharmacokinetics and pharmacodynamics of the prophylactic dose of enoxaparin once daily over 4 days in patients with renal impairment. Thromb Res 2002;105:225-31.

10. Schmid P, Brodman D, Fischer AG, et al. Study of bioaccumulation of dalteparin at a prophylactic dose in patients with various degrees of impaired renal function. JTH 2009;7:552-8.

11. Turpie AGG, Lensing AW, Fuji T, et al. Pharmacokinetic and clinical data supporting the use of fondaparinux 1.5 $\mathrm{mg}$ once daily in the prevention of venous thromboembolism in renally impaired patient. Blood Coagul Fibrinoly 2009;20:114-21. 


\title{
Sicurezza nella gestione della terapia anticoagulante orale
}

\author{
Micaela La Regina, ${ }^{1}$ Fulvio Pomero ${ }^{2}$ \\ ${ }^{1}$ SS Risk Management, ASL5 Liguria, La Spezia; ${ }^{2}$ S.C. Medicina Interna, ASL CN2, Alba-Bra (CN), Italia
}

\section{Introduzione}

Secondo i dati dell'Eudravigilance, gli antitrombotici (ATC B01) sono al $4^{\circ}$ posto nella classifica dei farmaci più spesso coinvolti negli errori in terapia farmacologia. ${ }^{1}$ Secondo lo studio recentemente pubblicato su JAMA, ${ }^{2}$ gli anticoagulanti sono i farmaci che più spesso determinano accesso in Pronto Soccorso e ricovero ospedaliero per eventi avversi $(17,7 \%$ di tutti gli accessi in PS e $48,8 \%$ di tutti i ricoveri ospedalieri per eventi avversi farmacologici.

Gli errori in terapia farmacologica sono tutti quegli eventi, non intenzionali, prevedibili che causano danno al paziente o determinano un uso inappropriato di un farmaco.

La maggior parte d egli studi che hanno indagato le tipologie degli errori coinvolgenti gli antitrombotici e le cause sono dedicati agli anticoagulanti piuttosto che agli antiaggreganti e l'ambiente ospedaliero più che il territorio.

È impressionante come i primi report sugli anticoagulanti orali diretti (DOACs) dimostrino che essi riducano alcune tipologie di eventi, ma non siano immuni dagli errori e che anche l'informatizzazione del processo non annulla gli errori. ${ }^{3}$

\section{Tipologie di eventi, setting e cause}

Gli errori possono intervenire in qualsiasi step del processo farmacologico, dalla prescrizione del far-

Corrispondente: Fulvio Pomero, U.O. Medicina Interna, Ospedale S. Lazzaro, via P. Belli 26, 12100 Alba (CN), Italia. Tel.: +39.0173316863 .

E-mail: fulviopomero@yahoo.it

Articolo pubblicato secondo la Creative Commons Attribution NonCommercial 4.0 License (CC BY-NC 4.0).

${ }^{\circ}$ Copyright: the Author(s), 2019

Licensee PAGEPress, Italy

QUADERNI - Italian Journal of Medicine 2019; 7(6):127-128 maco, all'ordinazione e dispensazione dalla farmacia, alla somministrazione.

I tipi di eventi più comuni sono le omissioni e la somministrazione di dosi eccessive. L'omissione è più facile che si verifichi in caso il farmaco non venga ordinato o non dispensato o non somministrato. ${ }^{4}$

A proposito della non somministrazione è più facile per il Warfarin perchè va somministrato ad orari fissi e sulla base del risultato dei test coagulativi giornalieri, quindi potrebbe avvenire che all'orario prestabilito, il risultato del test non sia disponibile e successivamente viene dimenticato. ${ }^{4}$

Altra causa di omissione è la mancata ripresa del farmaco in caso di temporanea sospensione per un INR eccessivo (warfarin) o per una procedura invasiva (warfarin/anticoagulanti diretti), una non completa ricognizione farmacologica all'ingresso o la mancata supervisione dell'assunzione della terapia nei pazienti collaboranti. ${ }^{4}$

Le dosi eccessive vengono erroneamente prescitte/somministrate quando il warfarin non viene sospeso pur a fronte di INR $>3$ o quando un qualsiasi anticoagulante viene duplicato (aggiunta di dose profilattica a dose terapeutica). ${ }^{4}$

Per quando riguarda le fasi del percorso ospedaliero di un paziente più spesso sede di errori sono le transizioni di cura (ingresso, dimissione, trasferimento di reparto e perioperatorio).

Gli studi che hanno indagato le cause-radice di questi errori hanno evidenziato come il fattore umano sia il maggior determinante $(53,4 \%$ dei casi), a seguito di deficit di performance (non applicare ciò che è corretto), mancata adesione a protocolli/linee guida, scarsa esperienza, omissione del doppio controllo. Deficit di comunicazione, organizzazione e tecnici intervengono rispettivamente nel 9,8\%,8,5\% e $5,4 \%$ dei casi. $^{5}$

\section{Strategie di prevenzione degli errori}

L'analisi degli eventi è fondamentale per definire le barriere da inserire nel processo farmacologico per ridurre/prevenire gli errori.

La Tabella 1 riassume le strategie raccomandate da applicare in tutti gli ospedali. ${ }^{4}$ 
Tabella 1. Strategie di prevenzione degli errori coinvolgenti gli anticoagulanti. ${ }^{4}$

\begin{tabular}{|c|c|}
\hline \multicolumn{2}{|l|}{ Strategie } \\
\hline Obbligatorie & $\begin{array}{l}\text { - Revisione da parte di un farmacista di ciascun ordine di farmaci prima della somministrazione (dose } \\
\text { e indicazione) } \\
\text { - Alert in caso di duplicazione } \\
\text { - No ordini verbali, salvo in caso di emergenza e con il metodo read back }\end{array}$ \\
\hline Standardizzazione & $\begin{array}{l}\text { - Standardizzazione delle informazioni di base prima della prescrizione (peso, creatinina clearance) } \\
\text { - Aggiustamento della dose correlato all'età } \\
\text { - Avviso per ordini in attesa } \\
\text { - Monitoraggio computerizzato INR } \\
\text { - Protocolli per l'inversione rapida di emergenza della terapia anticoagulante e il riavvio } \\
\text { - Evitare ordini incompleti e generici } \\
\text { - Evitare di riprendere tutte le medicine } \\
\text { - Evitare l'abbreviazione di NOAC fraintesa come NOAnticoagulazione } \\
\text { - Coordinamento prima del test di laboratorio e del tempo di amministrazione } \\
\text { - Consentire la med o la politica del paziente per la sostituzione se l'organizzazione riserva solo un } \\
\text { farmaco }\end{array}$ \\
\hline Controlli & $\begin{array}{l}\text { - Doppio controllo in farmacia e reparto } \\
\text { - Sistema di supporto decisionale clinico e sistema informativo farmaceutico } \\
\text { - Scansione del codice a barre durante il rifornimento e l'amministrazione delle scorte }\end{array}$ \\
\hline Coordinazione & $\begin{array}{l}\text { - Test di base disponibili in } 2 \text { ore prima di iniziare nuove terapie } \\
\text { - Disponibilità di andamento dell'INR }\end{array}$ \\
\hline Formazione del personale & $\begin{array}{l}\text { - Certificazione annuale delle competenze } \\
\text { - Newsletter e in-service e protocolli in caso di nuova introduzione anticoagulante } \\
\text { - Competenza certificata nella gestione della terapia anticoagulante }+ \text { antipiastrinici }\end{array}$ \\
\hline Educazione del paziente & $\begin{array}{l}\text { - Fondamentale perché il paziente possa contribuire a riconoscere, intercettare e prevenire errori } \\
\text { - Va eseguita all'inizio della terapia e almeno due giorni prima della dimissione }\end{array}$ \\
\hline Monitoraggio degli eventi avversi & - Essenziale per identificare le aree e le azioni di miglioramento \\
\hline
\end{tabular}

\section{Bibliografia}

1. https://www.ema.europa.eu/en/documents/presentation/presentation-data-analysis-based-overview-eudravigilance-data-victoria-newbould_en.pdf

2. Shehab N, et al. US Emergency Department Visits for Outpatient Adverse Drug Events, 2013-2014. JAMA 2016;316:2115-25.
3. Henriksen JN, et al. Medication errors involving anticoagulants: Data from the Danish patient safety database. Pharmacol Res Perspect 2017;5:e00307.

4. Andreica I, et al. Oral anticoagulants: a review of common errors and risk reduction strategies, Pennysilvania Patient Safety Authority, Vol. 12, No. 2-June 2015.

5. Dreijer AR, et al. Anticoagulant medication errors in hospitals and primary care: a cross-sectional study. Int J Qual Health Care 2018 [Epub ahead of print]. 


\title{
Strumenti per una gestione sicura delle terapie inalatorie
}

\author{
Claudia Tieri \\ Dirigente Medico di Pronto Soccorso, Ospedale San Paolo, Bari, Italia
}

\section{Introduzione}

Per gran parte delle malattie dell'apparato respiratorio il modo più efficace ed efficiente di somministrare farmaci è la via inalatoria. I vantaggi di tale via di somministrazione sono: i) il farmaco è convogliato direttamente agli organi bersaglio; ii) biodisponibilità topica del farmaco notevolmente aumentata; iii) diffusione sistemica estremamente diminuita; iv) richiesta di dosi molto basse del farmaco; v) rapida e potente attività terapeutica; vi) effetti collaterali nettamente inferiori rispetto a quelli determinati dalla somministrazione sistemica.

Un aerosol è un sistema costituito da più particelle, solide o liquide, che sono talmente piccole da essere stabilmente sospese in un mezzo gassoso (generalmente aria, ma anche ossigeno). La deposizione degli aerosol nelle vie aeree è condizionata da diversi parametri, alcuni dipendenti dal farmaco stesso, altri da caratteristiche anatomo-fisiologiche e patologiche individuali. Le tecnologie che utilizziamo in aerosolterapia sono di tre tipi: gli aerosol dosati pressurizzati (pMDI), gli inalatori a polvere secca (DPI) e i nebulizzatori pneumatici o ad ultrasuoni. L'efficacia terapeutica dipende da una serie di fattori che includono la corretta diagnosi, la scelta dei farmaci, l'efficienza del dispositivo e le modalità di inalazione. Il corretto utilizzo dei dispositivi per la terapia inalatoria è fondamentale per garantire l'efficacia della cura e la gestione della malattia. La letteratura evidenzia che circa un terzo dei pazienti compie, indipendentemente dal tipo di device, errori nelle manovre inalatorie. È invece fondamentale sapere che, in caso di utilizzo sba-

Corrispondente: Claudia Tieri, Dirigente Medico di Pronto Soccorso, Ospedale San Paolo, Bari, Italia.

E-mail: claudia.tieri@libero.it

Articolo pubblicato secondo la Creative Commons Attribution NonCommercial 4.0 License (CC BY-NC 4.0).

${ }^{\circ}$ Copyright: the Author(s), 2019

Licensee PAGEPress, Italy

QUADERNI - Italian Journal of Medicine 2019; 7(6):129-134 gliato del proprio inalatore, l'efficacia del trattamento può essere ridotta o addirittura compromessa. Porre una perfetta diagnosi di una malattia cronica non ha rilevanza se non vi è aderenza con il trattamento.

\section{Aderenza del paziente}

L'aderenza a lungo e a breve termine può essere influenzata da fattori sociali e dei pazienti stessi. ${ }^{1,2} \mathrm{La}$ mancata adesione alla terapia prescritta può essere classificata come non intenzionale o intenzionale, ${ }^{3}$ può comportare cattivi risultati clinici e un aumento dei costi sanitari: ${ }^{1}$

- Fattori non intenzionali: terapia non compresa correttamente: i) incomprensione regime farmacologico prescritto (scarsa comunicazione tra medico e paziente); ii) barriere linguistiche.

- Fattori intenzionali: comprendere la terapia ma non aderire correttamente. Convinzioni del paziente: i) non è realmente necessario assumere farmaci con regolarità; ii) non sono veramente malati; iii) farmaci troppo costosi; iv) preoccupazioni per gli effetti collaterali; v) percezione della mancanza di effetto dal farmaco. Stress paziente e stile di vita occupato: i) regimi aerosol complessi ed esigenti; ii) fattori psicologici (ad es. depressione).

L'impostazione di obiettivi e i piani d'azione sono due strumenti motivazionali che possono migliorare l'aderenza dei pazienti e possono essere discussi in una conversazione bidirezionale di supporto con il paziente durante l'approccio centrato sul paziente. Si noti che un esempio di non aderenza involontaria è una tecnica errata del dispositivo aerosol a causa di un equivoco del regime terapeutico prescritto, che può essere corretto attraverso l'addestramento del paziente. Oggi sul mercato non esiste un inalatore perfetto e a prova di errore. Di seguito gli errori comuni che possono verificarsi con ciascun tipo di dispositivo (Tabelle 1-4). ${ }^{3-5}$

\section{Controllo delle infezioni}

I generatori di aerosol possono essere contaminati da agenti patogeni del paziente, del care giver e ambientali. Alcuni studi hanno riscontrato un'ampia disparità 
di metodi di pulizia del nebulizzatore e una scarsa conoscenza delle linee guida sul controllo delle infezioni. In assenza di controllo delle infezioni, un generatore di aerosol sarà contaminato e potrebbe causare colonizzazione batterica nel tratto respiratorio. ${ }^{6,7-12}$

- Educazione e consapevolezza dei pazienti: è stato documentato che i generatori di aerosol utilizzati a casa sono spesso contaminati da batteri. ${ }^{8,13,14}$ Pertanto, l'importanza della pulizia e manutenzione dell'attrezzatura per l'aerosol terapia dovrebbe essere enfatizzata nei programmi di educazione sul controllo delle infezioni ${ }^{15}$ con pazienti e operatori sanitari attraverso istruzioni orali e scritte ripetute.

- Pulizia e manutenzione dei generatori di aerosol: le istruzioni di pulizia cambiano a seconda del tipo di generatori di aerosol.

\section{Inalatori a dosaggio metrico pressurizzato (pMDI)}

Il contenitore di plastica dei pMDI deve essere pulito almeno una volta alla settimana ${ }^{16,17}$ come da indicazioni di seguito elencate.

Pulizia del pMDI: i) pulire una volta alla settimana e quando necessario. Controllare e pulire il foro di erogazione; ii) pulire l'inalatore se presente polvere dentro o intorno al foro di erogazione; iii) rimuovere il contenitore pMDI dal contenitore di plastica in modo che non si bagni; iv) risciacquare il contenitore di plastica con acqua calda, scuotere e svuotare per rimuovere l'acqua in eccesso lasciando asciugare per tutta la notte.

\section{Dispositivi accessori per inalatori a dosaggio misurato (distanziatori)}

Quando si utilizza un distanziatore con un pMDI, deve essere pulito prima del primo utilizzo e quindi pulito periodicamente in base ai suggerimenti del produttore.

Pulizia del dispositivo accessorio: i) smontare il dispositivo per la pulizia; ii) immergere le parti in acqua calda con detergente liquido e agitare delicatamente entrambi i pezzi avanti e indietro; iii) scuotere per rimuovere l'acqua in eccesso; iv) lasciare asciugare le parti in posizione verticale durante la notte; v) non asciugare il distanziatore perché questo ridurrà la somministrazione della dose a causa della carica sta-

Tabella 1. Inalatori a dose dosata pressurizzata (pMDI).

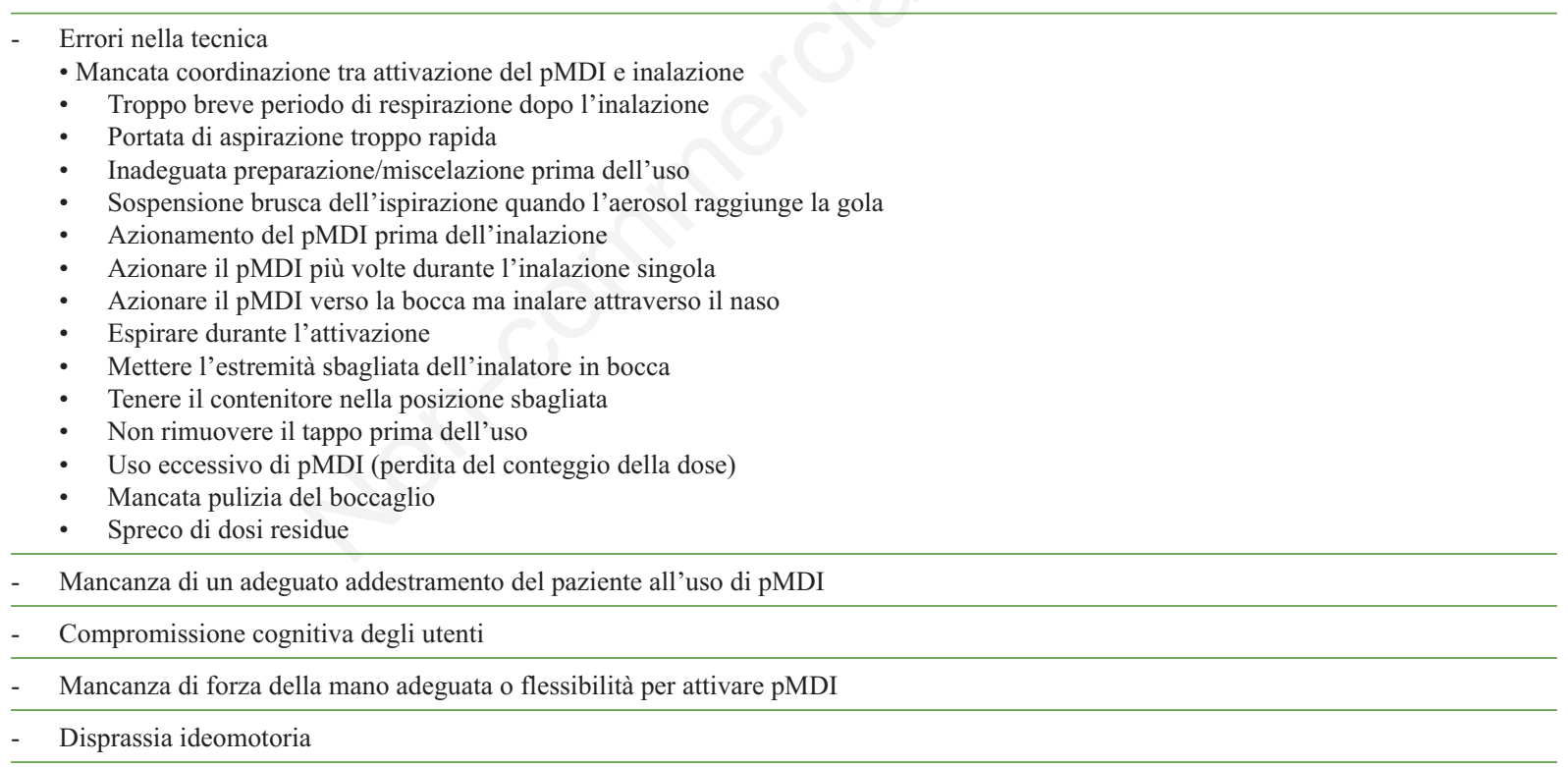

Tabella 2. Distanziatori Holding Chambers/Spacers.

\begin{tabular}{ll}
\hline & Assemblaggio errato del dispositivo \\
\hline- & Mancata rimozione della carica elettrostatica nei distanziatori non elettrostatici può ridurre la dose emessa nei nuovi distanziatori \\
\hline- & Ritardo tra l'attivazione del pMDI e l'inalazione dal distanziatore \\
\hline- & Inalazione troppo rapida \\
\hline & Attivazione di multipli puff nel distanziatore prima dell'inalazione \\
\hline & Mancanza di istruzioni per il paziente sull'assemblaggio o sull'uso \\
\hline
\end{tabular}


tica; vi) riposizionare il pezzo posteriore sul distanziatore quando è completamente asciutto.

\section{Inalatore con polvere secca}

È importante che i DPI non vengano immersi nell'acqua. Inoltre, dovrebbero essere mantenuti asciutti poiché l'umidità diminuirà la somministrazione del farmaco. Sebbene non ci siano prove chiare circa la pratica di pulizia DPI, ogni azienda manifatturiera ha raccomandazioni per la pulizia periodica e suggerisce di pulire il boccaglio del DPI con un panno pulito e asciutto.

\section{Nebulizzatori}

In casa, i nebulizzatori devono essere puliti dopo ogni trattamento. Uno studio ha mostrato che il $73 \%$ dei nebulizzatori era contaminato da microrganismi e il $30 \%$ aveva batteri potenzialmente patogeni. ${ }^{18}$ Inoltre, c'era un significativo aumento del rischio di esacerbazione della BPCO nei pazienti con BPCO che avevano nebulizzatori con agenti patogeni rispetto a quelli con batteri non patogeni. Tuttavia, la contaminazione del nebulizzatore può essere migliorata in modo significativo seguendo le regole sul controllo dell'infezione. ${ }^{19}$ Più un nebulizzatore è sporco e si asciuga più a lungo, più è difficile pulirlo a fondo. Risciacquare e lavare il nebulizzatore immediatamente dopo ogni trattamento può contribuire notevolmente a ridurre il rischio di infezione. Di seguito le istruzioni per la pulizia del nebulizzatore a getto.

Pulizia dopo ogni utilizzo: i) lavarsi le mani prima di maneggiare l'attrezzatura; ii) smontare le parti dopo ogni trattamento; iii) rimuovere il tubo dal compressore e metterlo da parte. il tubo non deve essere lavato o sciacquato; iv) risciacquare l'ampolla del nebulizzatore e il boccaglio con acqua sterile o acqua distillata; v) scuotere l'acqua in eccesso; vi) asciugare all'aria su un asciugamano assorbente; vii) conservare l'apparecchio in una busta di plastica con cerniera.

Pulizia una volta o due volte alla settimana: i) lavarsi le mani prima di maneggiare l'attrezzatura; ii) smontare le parti dopo ogni trattamento; iii) rimuovere il tubo dal compressore e metterlo da parte; iv) il tubo non deve essere lavato o sciacquato; v) lavare i componenti del nebulizzatore in acqua tiepida con detersivo liquido per piatti; vi) disinfettare il nebulizzatore in base alle raccomandazioni del produttore [Le parti del nebu-

Tabella 3. Dispositivi inalatori.

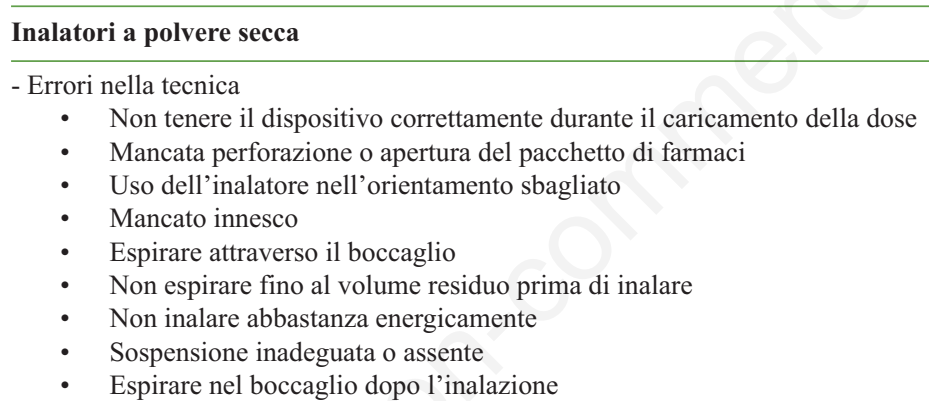

- Uso di modelli con serbatoi a più dosi in ambienti molto umidi, può ridurre la dose di particelle fini

- Mancanza di istruzioni per il paziente sull'assemblaggio o sull'uso

Nebulizzatori

- Mancato assemblaggio dell'attrezzatura

- Perdita della dose inclinando alcuni nebulizzatori

- Errato posizionamento del boccaglio durante la nebulizzazione

- Mancato respiro alla bocca

Tabella 4. Istruire e valutare i pazienti nell'uso di dispositivi inalatori.

Le seguenti misure generali sono raccomandate ai medici per garantire al paziente un uso corretto del dispositivo:

1. Esaminare attentamente le istruzioni del dispositivo ed esercitarsi con un dispositivo placebo prima di insegnare ad altri

2. Dimostrare il montaggio e l'uso corretto del dispositivo ai pazienti utilizzando una checklist

3. Fornire al paziente istruzioni scritte su come utilizzare il dispositivo e includere un piano scritto per l'uso del farmaco. Le istruzioni scritte dovrebbero essere accompagnate da immagini per i pazienti con basso livello di alfabetizzazione

4. Invitare il paziente a utilizzare il dispositivo mentre viene osservato dal medico

5. Rivedere l'uso del dispositivo da parte del paziente ad ogni visita di controllo 
lizzatore possono essere immerse in una delle seguenti soluzioni: 1. Soluzione 50:1 di acqua e candeggina per tre minuti; 2 . Alcool isopropilico al $70 \%$ per cinque minuti. 3. Perossido di idrogeno al 3\% per 30 minuti. 4 . Soluzione 1:3 di aceto bianco distillato e acqua calda per un'ora.]; vii) lavare le parti con acqua sterile o distillata; viii) scuotere l'acqua in eccesso e posizionare tutte le parti su un tovagliolo di carta pulito; ix) lasciare asciugare completamente all'aria su un asciugamano assorbente; $\mathrm{x}$ ) riassemblare il nebulizzatore e riporlo in un sacchetto-contenitore pulito e asciutto.

I nebulizzatori a ultrasuoni devono essere puliti e disinfettati in base alle raccomandazioni del produttore. Ogni produttore suggerisce un diverso metodo di disinfezione per il suo prodotto. Pertanto, devono essere seguite le istruzioni specifiche del produttore sulla disinfezione dei generatori di aerosol. È anche importante notare che tutte le soluzioni devono essere eliminate dopo la disinfezione.

\section{Metodi di disinfezione}

Metodi a caldo: i) far bollire o porre nel microonde ${ }^{20}$ le parti del nebulizzatore per cinque minuti. Questo processo di disinfezione non richiede un risciacquo finale; ii) lavare in lavastoviglie se la lavastoviglie raggiunge una temperatura $>158^{\circ} \mathrm{F} \mathrm{o} 70^{\circ} \mathrm{C}$ per $30 \mathrm{~min}^{20}$

Metodi a freddo i) soluzione 50:1 di acqua e candeggina per tre minuti; ii) alcool isopropilico al $70 \%$ per cinque minuti; iii) perossido di idrogeno al $3 \%$ per 30 minuti; iv) soluzione 1:3 di aceto bianco distillato e acqua calda per un'ora.

Risciacquo finale: il paziente deve usare acqua sterile (non distillata o imbottigliata) per il risciacquo finale. ${ }^{21}$ L'acqua sterile può essere ottenuta bollendo l'acqua del rubinetto per cinque minuti.

Asciugatura e manutenzione: poiché i batteri crescono in luoghi bagnati e umidi, i nebulizzatori devono essere accuratamente asciugati e conservati in un luogo pulito e asciutto tra un trattamento e l'altro. L'asciugatura può essere migliorata collegando il flusso di gas al nebulizzatore per un breve periodo dopo il risciacquo. È stato segnalato che le prestazioni del nebulizzatore possono cambiare nel tempo a causa di procedure errate di pulizia, manutenzione e disinfezione. ${ }^{22}$ I nebulizzatori devono essere mantenuti decontaminati seguendo le istruzioni del produttore per la cura e la pulizia. Questo è necessario per tutti i generatori di aerosol terapia.

\section{Prevenire l'infezione e il malfunzionamento dei generatori di aerosol presso ospedali o cliniche}

Generatori di aerosol: La contaminazione batterica dei nebulizzatori in ospedale è stata associata a infezioni nosocomiali. ${ }^{23,24}$ I Centri per il controllo e la prevenzione delle malattie raccomandano che i nebulizzatori vengano puliti, risciacquati con acqua sterile e asciugati all'aria tra un trattamento e l'altro. Inoltre, ogni clinica ospedaliera o ambulatorio dovrebbe disporre di un programma di sorveglianza delle infezioni in grado di determinare le pratiche locali di controllo delle infezioni basate sulla raccolta, analisi e interpretazione continua e sistematica dei dati di infezione. I nebulizzatori dovrebbero essere sostituiti ogni 24 ore. ${ }^{25,26}$

Farmaci per via inalatoria: le confezioni di farmaci multi-dose sono stati associati a nebulizzatori contaminati e rappresentano una potenziale fonte di diffusione delle infezioni nosocomiali..$^{27,28}$ Pertanto, quando possibile, si raccomandano farmaci monodose. ${ }^{29}$ Inoltre, è importante evitare di contaminare le soluzioni farmacologiche.

Trasmissione dell'infezione: la trasmissione dell'infezione dal sanitario al paziente può essere ridotta lavandosi le mani con acqua e sapone o utilizzando disinfettanti per le mani prima e dopo il trattamento. ${ }^{28-30}$ L'uso dei guanti deve essere considerato un'aggiunta all'igiene delle mani. Bisognerebbe cambiare i guanti tra un paziente e l'altro e lavarsi le mani dopo che i guanti sono stati rimossi perché i guanti creano un ambiente caldo e umido che supporta la crescita della contaminazione microbica e, quindi, la trasmissione dell'infezione. ${ }^{31,32}$ Occhiali protettivi e maschere facciali dovrebbero essere usati da soli o in combinazione come barriera nei confronti dei patogeni presenti nell'aria che i sanitari potrebbero inalare con la terapia aerosolica.

Conformità di gestione del sistema di controllo delle infezioni: il sistema di gestione del controllo delle infezioni può essere efficace solo grazie a personale esperto e competente. Pertanto, le figure sanitarie coinvolte dovrebbero essere adeguatamente addestrate all'uso dei protocolli stabiliti.

Sorveglianza delle infezioni: è essenziale che gli ospedali stabiliscano misure di sorveglianza delle infezioni semplici e sensibili per valutare periodicamente le attività di controllo delle infezioni utilizzate dai sanitari.

\section{Salute e sicurezza sul lavoro dei sanitari}

I sanitari, nell'ambito del contesto clinico correlato con patologie respiratorie, non subiscono solo il rischio di esposizione a farmaci per inalazione, ma devono anche affrontare il rischio di inalazione di agenti patogeni durante l'aerosol terapia.

Gli elementi di salute e sicurezza sul lavoro per i sanitari sono riportati di seguito.

Valutazione della salute e immunizzazione: i sanitari dovrebbero essere screenati per infezioni e immunizzazione dall'inizio alla fine del rapporto di lavoro. 
Igiene delle mani: è stato documentato che l'igiene delle mani è efficace nel ridurre la trasmissione dei virus respiratori..$^{30,33-36}$ Inoltre, le linee guida dell'Organizzazione Mondiale della Sanità suggeriscono che l'inadeguata igiene delle mani è la principale causa di infezione nosocomiale e diffusione di organismi multi-farmaco resistenti. ${ }^{37}$ Gli operatori sanitari che hanno riferito di aver lavato le mani durante la cura dei pazienti hanno avuto un rischio inferiore di contrarre infezioni respiratorie. . $^{33,34-36}$

Dotazione di presidi di protezione: i sanitari devono avere accesso all'apposito dispositivo di protezione personale, come maschere e protezioni per gli occhi, quando necessario. ${ }^{31}$ Sistema di ventilazione: questi sistemi scambiano l'aria nella stanza da sei a 10 volte all'ora 31 e creano un ambiente a pressione negativa nelle stanze dei pazienti che è efficace nell'eliminare il $99,9 \%$ dei contaminanti presenti nell'aria in 69 minuti. ${ }^{38}$

Filtri per nebulizzatori: l'inserimento di un filtro sulla parte espiratoria di un nebulizzatore può proteggere i sanitari dall'infezione e ridurre la respirazione della terapia aerosolica espirato negli ospedali e nelle cliniche ambulatoriali.

\section{Bibliografia}

1. Braido F, Baiardini I, Blasi F, et al. Adherence to asthma treatments: 'we know, we intend, we advocate'. Curr Opin Allergy Clin Immunol 2015;15:49-55.

2. Bourbeau J, Bartlett SJ. Patient adherence in COPD. Thorax 2008;63:831-8.

3. Rau JL. Determinants of patient adherence to an aerosol regimen. Respir Care 2005;50:1346-59.

4. Melani AS, Zanchetta D, Barbato N, et al. Inhalation technique and variables associated with misuse of conventional metered-dose inhalers and newer dry powder inhalers in experienced adults. Ann Allergy Asthma Immunol 2004;93:439-46.

5. McFadden ER Jr. Improper patient techniques with metered-dose inhalers: clinical consequences and solutions to misuse. J Allergy Clin Immunol 1995;96:278-83.

6. Pitchford K, Corey M, Highsmith A, et al. Pseudomonas species contamination of cystic fibrosis patients' home inhalation equipment. J Pediatr 1987;111:212-6.

7. Vassal S, Taamma R, Marty N, et al. Microbiologic contamination study of nebulizers after aerosol therapy in patients with cystic fibrosis. Am J Infect Control 2000;28:347-51.

8. O'Callaghan C, Barry PW. The science of nebulised drug delivery. Thorax 1997;52:S31-44.

9. Craven DE, Lichtenberg DA, Goularte TA, et al. Contaminated medi-cation nebulizers in mechanical ventilator circuits. Source of bacterial aerosols. Am J Med 1984;77:834-8.

10. Lester MK, Flume PA, Gray SL, et al. Nebulizer use and maintenance by cystic fibrosis patients: a survey study. Respir Care 2004;49:1504-8.

11. Garber E, Desai M, Zhou J, et al. Barriers to adherence to cystic fibrosis infection con trol guidelines. Pediatr Pulmonol 2008;43:900-7.

12. Jakobsson BM, Onnered AB, Hjelte L, Nystrom B. Low bacterial contam-ination of nebulizers in home treatment of cystic fibrosis patients. J Hosp Infect 1997;36:201-7.

13. Cohen HA, Kahan E, Cohen Z, et al. Microbial colonization of nebulizers used by asthmatic children. Pediatr Int 2006;48:454-8.

14. Blau H, Mussaffi H, Mei Zahav M, et al. Microbial contamination of neb-ulizers in the home treatment of cystic fibrosis. Child Care Health Dev 2007;33:491-5.

15. Saiman L, Siegel J. Infection control in cystic fibrosis. Clin Microbiol Rev 2004;17:57-71.

16. Chew NY, Reddel HK, Bosnic-Anticevich SZ, Chan HK. Effect of mouth-piece washing on aerosol performance of CFC-free Ventolin. J Asthma 2004;41:721-7.

17. American College of Chest Physicians. Priming and cleaning your MDI and spacer, 2006.

18. Jarvis S, Ind PW, Thomas C, et al. Microbial contamination of domicil-iary nebulisers and clinical implications in chronic obstructive pulmo-nary disease. BMJ Open Respir Res 2014;1:e00018.

19. Della Zuana A, Garcia Dde O, Juliani RC, et al. Effect that an educa-tional program for cystic fibrosis patients and caregivers has on the contamination of home nebulizers. J Bras Pneumol 2014;40:119-27.

20. Saiman L, Siegel JD, LiPuma JJ, et al. Infection prevention and con-trol guideline for cystic fibrosis: 2013 update. Infect Control Hosp Epidemiol 2014;35:S1-67.

21. The Cystic Fibrosis Foundation. Stopping the spread of germs; 2009.

22. Le Brun PP, de Boer AH, Heijerman HG, Frijlink HW. A review of the technical aspects of drug nebulization. Pharm World Sci 2000;22:75-81.

23. Grieble HG, Colton FR, Bird TJ, et al. Fine-particle humidifiers. Source of Pseudomonas aeruginosa infections in a respiratory-disease unit. $\mathrm{N}$ Engl $\mathrm{J}$ Med 1970;282:531-35.

24. Mertz JJ, Scharer L, McClement JH. A hospital outbreak of Klebsiella pneumonia from inhalation therapy with contaminated aerosol solutions. Am Rev Respir Dis 1967;95:454-60.

25. O'Malley CA, VandenBranden SL, Zheng XT, et al. A day in the life of a nebulizer: surveillance for bacterial growth in nebulizer equipment of children with cystic fibrosis in the hospital setting. Respir Care 2007;52:258-62.

26. American Association for Respiratory Care. AARC Clinical Practice Guideline: selection of an aerosol delivery device. Respir Care 1992;37:891-7 (retired August 2006).

27. Rau JL, Restrepo RD. Nebulized bronchodilator formulations: unit-dose or multi-dose? Respir Care 2003;48:926-39.

28. Center for Disease Control and Prevention. Clean hands save lives; 2008.

29. Tablan OC, Anderson LJ, Besser R, et al. Guidelines for preventing health care-associated pneumonia, 2003: recommendations of CDC and the Healthcare Infection Control Practices Advisory Committee. MMWR Recomm Rep 2004;53:1-36.

30. Center for Disease Control and Prevention. Guideline for hand hygiene in healthcare settings; 2008.

31. Rhinehart E, Friedman MM. Personal protective equip- 
ment and staff supplies. In: Infection control in home care (An official APIC publica-tion). Gaithersburg, MD: Aspen Publishers, Inc.; 2006. pp 61-69.

32. Larson EL. APIC guideline for handwashing and hand antisepsis in health care settings. Am J Infect Control 1995;23:251-69.

33. Gamage B, Moore D, Copes R, et al. Protecting health care workers from SARS and other respiratory pathogens: a review of the infection control literature. Am J Infect Control 2005;33:114-21.

34. Paes BA. Current strategies in the prevention of respiratory synctial virus disease. Pediatr Respir Rev 2003;4:21-7.
35. Purssell E. Preventing nosocomial infection in paediatric wards. J Clin Nurs 1996;5:313-8.

36. Hall CB. Nosocomial respiratory syncytial virus infections: the "Cold War" has not ended. Clin Infect Dis 2000;31:590-6.

37. World Health Organization (WHO). WHO Guidelines on Hand Hygiene in Health Care: First Global Patient Safety Challenge Clean Care Is Safer Care. World Health Organization: Geneva; 2009.

38. Segal-Maurer S, Kalkut G. Environmental control of tuberculosis: continuing controversy. Clin Infect Dis 1994;19:299-308. 


\title{
Strumenti per una gestione sicura della terapia ipoglicemizzante
}

\author{
Tiziana Marcella Attardo
}

Direzione Medica, Diabetologia e Malattie del Metabolismo, UO di Medicina Interna, Ospedale Barone Lombardo, Canicattì (AG), Italia

\section{Introduzione}

Un corretto management della cura della malattia diabetica riguarda molteplici ambiti: dalla prevenzione dell'ipoglicemia (basata sulla scelta di farmaci che siano a più basso rischio ipoglicemizzante) alla corretta formazione ed educazione terapeutica del paziente e/o del caregiver, addestrati ad eseguire un adeguato automonitoraggio glicemico ed una corretta auto somministrazione, cosi da sviluppare l'empowerment alla malattia.

\section{Ridurre il rischio di ipoglicemie}

La prevenzione del rischio di ipoglicemia è uno degli obiettivi principali da perseguire nella terapia, soprattutto se intensiva e se gli obiettivi glicemici sono ambiziosi. Ottenere un buon compenso glicemico significa, infatti, non solo raggiungere una normoglicemia, ma anche ridurre al minimo il rischio di ipoglicemia ed evitare le eccessive variazioni glicemiche (variabilità glicemica).

Il trattamento anti-iperglicemizzante, in primis la terapia insulinica e quella a base di sulfoniluree, è gravato dal rischio di ipoglicemia che rappresenta la complicanza più temibile nel trattamento del paziente con diabete mellito. In generale, la frequenza dell'ipoglicemia è certamente più alta nel diabete mellito di tipo 1 rispetto a quello di tipo $2 .{ }^{1}$ In letteratura gli eventi di ipoglicemia grave nel diabete di tipo 2 variano da 3 a 73 episodi per 100 pazienti-anno, mentre nel diabete

Corrispondente: Tiziana Marcella Attardo, Direzione Medica, Diabetologia e Malattie del Metabolismo, UO di Medicina Interna, Ospedale Barone Lombardo, Canicattì (AG), Italia. E-mail: claudia.fantucchio@alice.it

Articolo pubblicato secondo la Creative Commons Attribution NonCommercial 4.0 License (CC BY-NC 4.0).

${ }^{\circ}$ Copyright: the Author(s), 2019

Licensee PAGEPress, Italy

QUADERNI - Italian Journal of Medicine 2019; 7(6):135-144 di tipo 1 variano dal 62 a 320 episodi per 100 pazientianno. ${ }^{1}$ In generale, poi, il rischio di ipoglicemia aumenta con l'avanzare dell'età, indipendentemente dal grado di controllo glicemico, sia nel diabete mellito di tipo 1 che di tipo $2,{ }^{2}$ ed è correlato all'intensificazione del controllo glicemico (sia nel diabete di tipo $1^{3}$ che di tipo $\left.2^{4-6}\right)$, alla durata di malattia e all'uso di insulina e sulfoniluree.

L'ipoglicemia può causare una rilevante compromissione delle attività intellettuali e fisiche del paziente $\mathrm{e}$, se prolungata e/o grave, determinare convulsioni, coma, danno neurologico permanente e perfino morte, a seguito di una marcata neuroglicopenia. Inoltre aumenta il rischio di eventi cardiovascolari, ${ }^{7,8}$ di demenza, ${ }^{9}$ di fratture a seguito di cadute ${ }^{10} \mathrm{e}$ di mortalità in generale. ${ }^{11}$ Essa peggiora la qualità della vita, ${ }^{12-13}$ genera paura nei confronti della terapia antiiperglicemizzante ${ }^{14} \mathrm{e}$ pertanto è concausa di mancato raggiungimento di buon controllo metabolico. Naturalmente i soggetti maggiormente esposti al rischio di ipoglicemia grave sono quelli più anziani, con ridotta funzione renale, fragili, comorbidi e politrattati.

È evidente che l'ipoglicemia grava in senso peggiorativo, anche, sui costi diretti ed indiretti della cura del diabete: tutto questo, oltre a impattare negativamente sulla qualità di vita del paziente, comporta, infatti, anche un notevole aggravio dei costi della spesa sanitaria. Per quanto riguarda il costo dell'ipoglicemia, in particolare di un ricovero per ipoglicemia grave, esso ammonta a circa 4000-6000 € considerando che una giornata di degenza costa circa $700 €$ e la durata media di degenza e di 6 giorni. ${ }^{15,16}$ A questa cifra del costo diretto, vanno aggiunti anche i costi del Servizio Emergenza e le prestazioni in Pronto Soccorso (220 e $245 €$, rispettivamente), ${ }^{15,16}$ e una quota variabile non facilmente calcolabile di costi indiretti (assenza dal lavoro, impegno di familiari, persone terze, ecc.). Si tratta di un costo certamente elevato (e sottostimato), vale a dire del $70-100 \%$ maggiore rispetto al costo per le cure per una persona con diabete in un anno, considerando che la spesa media pro capite/anno per una persona con diabete è pari a circa $2898 € .{ }^{17}$ In altre parole, prevenendo un ricovero per ipoglicemia grave, si rendono disponibili risorse economiche per curare 2 persone con diabete per un intero anno. 
Partendo dall'assunto che la terapia prima di essere efficace deve essere sicura, la versione aggiornata del 2018 degli Standard di Cura del Diabete ${ }^{18}$ declina l'obiettivo di emoglobina glicata da raggiungere, anche, in relazione alla terapia farmacologica adottata. Così, laddove si preveda l'impiego di farmaci potenzialmente rischiosi per ipoglicemia (insulina, sulfaniluree o glinidi) vi è indicazione a mantenere l'obiettivo di emoglobina glicata da raggiungere a livelli più elevati (tra 6,5 e 7,5\%). In caso di impiego di farmaci in grado di causare ipoglicemia, è bene anche tener conto della presenza di condizioni che ne possano aumentare ulteriormente il rischio (infanzia ed adolescenza, età molto avanzata, presenza di comorbilità). In questi casi, può essere opportuno mantenere l'emoglobina glicosilata a livelli relativamente più elevati, fino ad un massimo di 8,0\%. Al contrario, nei casi di diabete non complicato e trattati con farmaci a basso rischio di ipoglicemia si potrà spingere l'obiettivo di emoglobina glicosilata da raggiungere al $6,5 \%$.

È indubbio che tutte le scelte nella terapia ipoglicemizzante, personalizzata e fenotipizzata, debbano essere obbligatoriamente guidate dal profilo e dalla storia clinica del singolo paziente.

Età, durata della malattia, presenza delle complicanze croniche del diabete, di malattia cardiovascolare o comunque di un alto rischio cardiovascolare, di altre comorbidità, nonché l'aspettativa di vita sono tra i principali aspetti da tenere in considerazione quando si definiscono percorsi di intervento personalizzato. Questi percorsi devono coniugare obiettivi di controllo glicometabolico possibili e sicuri con scelte terapeutiche in grado di garantire il migliore compromesso tra efficacia, sicurezza e aderenza terapeutica. ${ }^{19}$

In una buona percentuale dei casi, dunque, l'ipoglicemia causata dai farmaci può essere prevenuta semplicemente evitando l'utilizzo di farmaci che potenzialmente possono indurre ipoglicemie, valutando la presenza dei fattori di rischio dell'ipoglicemia, in particolare l'età avanzata, la fragilità, la funzionalità renale, e individualizzando target glicemici meno stringenti.

D'altra parte da sempre anche gli Standard Italiani di Cura del Diabete SID-AMD ${ }^{20}$ hanno richiamato l'attenzione sul rischio dell'uso delle sulfaniluree, in particolare della glibenclamide, che è controindicata nell'anziano non solo per una lunga durata d'azione e rischio di ipoglicemia grave, ma anche per un possibile rischio cardiovascolare e di aumento della mortalità. ${ }^{21}$ Pertanto, sulla base di queste evidenze, la glibenclamide non deve più essere utilizzata, come pure le altre sulfoniluree che condividono lo stesso perverso meccanismo d'azione (stimolazione della secrezione insulinica non glucosio-mediata). Questo giudizio vale anche per le glinidi (repaglinide) che pure possono indurre severe ipoglicemie.
Da qualche anno (dal 2008 in Italia) sono in commercio delle nuove molecole, le incretine, che sebbene comportino una spesa media per paziente trattato per anno decisamente più elevata, rispetto a quella degli ipoglicemizzzanti tradizionali (exenatide $749 €$ /anno, gliptine $338 €$ circa/anno), ${ }^{17}$ si caratterizzano per efficacia nel mantenere un buon controllo glicemico nel tempo e per sicurezza, in termini di riduzione delle ipoglicemie, anche in pazienti con età superiore ai 75 anni.

Per quanto concerne, poi, i casi ricoverati per ipoglicemia grave in trattamento insulinico, in particolare per i pazienti anziani e fragili, oltre a tenere conto anche della presenza dei fattori di rischio principali come l'hypoglycemia unawareness e la variabilità glicemica, certamente l'educazione terapeutica, ossia, la corretta modalità di somministrazione dell'insulina, la giusta dose da adeguare al pasto e l'automonitoraggio domiciliare, dovrebbe essere estesa anche a familiari ed assistenti dei soggetti anziani, per ridurre errori di somministrazione e di sovradosaggio insulinico, causa di eventi avversi, riospedalizzazioni, continui ricorsi al Pronto Soccorso ed incremento dei ricoveri inappropriati.

Oggi, le innovazioni in ambito di terapia insulinica, ci permettono di optare verso analoghi rapidi, ultrarapidi e lenti insulinici che riducono notevolmente il rischio di ipoglicemie, diurne e notturne.

\section{Automonitoraggio glicemico}

Nel 2013, a 26 anni dalla promulgazione della L $115 / 87$, è stato portato a compimento il Piano Nazionale Diabete (PND) redatto dal Ministero della Salute quale strumento utile a promuovere e supportare le attività rivolte alla prevenzione, diagnosi e cura della patologia diabetica, permettendo uno scambio e un ruolo di stimolo e controllo nei confronti di Regioni, decisori e governance sanitaria. ${ }^{22}$ Tra i piani redatti dal Ministero della Salute, questo è stato il primo dedicato a una specifica malattia ed è nato nell'ottica della evidence based practice, secondo i principi delle buone pratiche da valorizzare, condividere e disseminare. Alla sua stesura hanno contribuito associazioni di pazienti, associazioni professionali e società Scientifiche. Il Piano sulla malattia diabetica identifica dieci obiettivi generali, che focalizzano l'attenzione su alcune aree chiave, al fine di ridurre l'impatto della malattia sulle persone con o a rischio di diabete, sulla popolazione e sul SSN.

Come si può evincere dalla Tabella 1, il Piano propone una gestione efficace ed efficiente della malattia che presuppone la sicurezza della stessa; inoltre aumentare e diffondere le competenze tra gli operatori della rete assistenziale favorendo lo scambio continuo di informazioni per una gestione efficace ed efficiente, centrata sulla persona e la Promozione dell'uso appro- 
priato delle tecnologie, al fine di sviluppare l'empowerment delle persone con diabete e delle comunità.

Quali, quindi, gli strumenti disponibili al clinico per permettere una gestione efficace e sicura delle cure del diabete mellito?

L'automonitoraggio glicemico domiciliare è certamente parte integrante della terapia del diabete mellito e della sicurezza della cura. Esso permette, infatti, una autogestione del controllo glicemico, riuscendo il paziente, ben addestrato ed educato, a modificare la terapia in relazione ai valori glicemici rilevati e a prevenire/controllare le ipoglicemie.

Per ciò che riguarda il ruolo della tecnologia è significativa la crescente diffusione dei nuovi device per il monitoraggio in continuo del glucosio e dei microinfusori insulinici, caratterizzati da sistemi sempre più sofisticati di automatizzazione della terapia infusiva, con indubbi vantaggi sia in termini clinici, di qualità di vita, che di natura economica.

La misurazione della concentrazione di glucosio nel sangue è un elemento essenziale per la gestione della malattia diabetica, perché consente di adeguare la dose dei farmaci ipoglicemizzanti, di monitorare l'efficacia della terapia e di verificare le oscillazioni glicemiche. La disponibilità di strumenti in grado di misurare la concentrazione di glucosio nel liquido interstiziale, attraverso un ago-sensore inserito sottocute, consente di misurare continuativamente la concentrazione di glucosio nel fluido interstiziale, inviando i valori registrati a un monitor esterno. I dati derivanti dal monitoraggio in continuo del glucosio (continuous glucose monitoring, CGM) possono essere visualizzati retrospettivamente, dopo che il paziente ha indossato il sensore (CGM retrospettivo), oppure in tempo reale, con la visualizzazione immediata del valore di glucosio e dei grafici dell'andamento glicemico delle ultime ore (real-time CGM, RTCGM). L'analisi dei dati del sensore può mettere in luce come l'alimentazione, l'esercizio fisico, i farmaci e lo stile di vita influiscano sui livelli glicemici, mettendo i pazienti nella condizione di prendere decisioni più consapevoli ed acquisire un maggiore senso di fiducia nell'autogestione della terapia. ${ }^{23}$

I dispositivi CGM devono essere calibrati periodicamente con il corrispondente valore di glicemia capillare ottenuto con il self monitoring of blood glucose $(\mathrm{SMBG}){ }^{23}$

L'uso del CGM è stato finora inteso come aggiuntivo e non sostitutivo del SMBG: un paziente che indossa un sensore CGM deve verificare il valore della glicemia capillare (mediante SMBG) tutte le volte che il valore del glucosio letto dal dispositivo interstiziale sia in rapida variazione, quando sia necessario prendere una decisione terapeutica (ad esempio somministrazione di insulina), quando il valore letto dal sensore non sia corrispondente ai sintomi percepiti (ad esempio ipoglicemia). Recentemente, dopo che lo studio REPLACE-BG ha documentato che l'uso di un dispositivo RT-CGM senza la conferma del SMBG non ha determinato peggioramento dell' $\mathrm{HbA} 1 \mathrm{c}$ o aumento del rischio di ipoglicemie ${ }^{24}$ ad uno specifico sensore (Dexcom G5) è stata riconosciuta la possibilità di uso non aggiuntivo a SMBG. ${ }^{25}$ L'evoluzione dei dispositivi e degli algoritmi che questi utilizzano apre quindi

\section{Tabella 1. Obiettivi del Piano della malattia diabetica. ${ }^{22}$}

1. Migliorare la capacità del SSN nell'erogare e monitorare i Servizi, attraverso l'individuazione e l'attuazione di strategie che perseguano la razionalizzazione dell'offerta e che utilizzino metodologie di lavoro basate sopratutto sull'appropriatezza delle prestazioni erogate

2. Prevenire o ritardare l'insorgenza della malattia attraverso l'adozione di idonei stili di vita; identificare precocemente le persone a rischio e quelle con diabete; favorire adeguate politiche di intersettorialità per la popolazione generale $\mathrm{e}$ a rischio e per le persone con diabete

3. Aumentare le conoscenze circa la prevenzione, la diagnosi, il trattamento e l'assistenza, conseguendo,, attraverso il sostegno alla ricerca, progressi di cura, riducendo le complicanze e la morte prematura

4. Rendere omogenea l'assistenza, prestando particolare attenzione alle disuguaglianze sociali e alle condizioni di fragilità e/o vulnerabilità socio-sanitaria sia per le persone a rischio che per quelle con diabete

5. Nelle donne diabetiche in gravidanza raggiungere outcome materni e del bambino equivalenti a quelle delle donne non diabetiche; promuovere iniziative finalizzate alla diagnosi precoce nelle donne a rischio; assicurare la diagnosi e l'assistenza alle donne con diabete gestazionale

6. Migliorare la qualità di vita e della cura e la piena integrazione sociale per le persone con diabete in età evolutiva anche attraverso strategie di coinvolgimento familiare

7. Organizzare e realizzare le attività di rilevazione finalizzate alla programmazione e al miglioramento dell'assistenza, per una gestione efficace ed efficiente della malattia

8. Aumentare e diffondere le competenze tra gli operatori della rette assistenziale favorendo lo scambio continuo di informazioni per una gestione efficace ed efficiente, centrata sulla persona

9. Promuovere l'appropriatezza nell'uso delle tecnologie

10. Favorire varie forme di partecipazione, in particolare attraverso il coinvolgimento di Associazioni riconosciute di persone con diabete, sviluppando l'empowerment delle persone con diabete e delle comunità 
uno scenario in cui le misurazioni del glucosio rilevate da questi sensori potranno essere utilizzate per aggiustamenti terapeutici. ${ }^{23}$

Nel complesso, le valutazioni metanalitiche suggeriscono che rispetto al SMBG, il CGM riduce la HbAlc dello $0,3 \%$ circa e il tempo speso in iperglicemia. $^{26,27}$

Il CGM in diversi studi ha contribuito a ridurre le ipoglicemie e le iperglicemie facilitando il loro precoce riconoscimento e l'intervento correttivo/preventivo. ${ }^{28-32}$

Le valutazioni di costo-efficacia relativamente all'uso del CGM sono molto limitate, e indicano un rapporto complessivamente favorevole nei pazienti in cui si ottenga una significativa riduzione dell' $\mathrm{HbA} 1 \mathrm{c}$ e del rischio di ipoglicemie severe. ${ }^{23}$

Una nuova modalità di monitoraggio del glucosio è il cosiddetto Flash Glucose Monitoring (FGM), una rilevazione del glucosio interstiziale on demand, cioè a richiesta del paziente. Si tratta di un sistema che utilizza un sensore ad ago inserito sottocute e sostituito ogni 14 giorni, in grado di leggere le concentrazioni di glucosio nel liquido interstiziale. Il sensore FGM attualmente disponibile non necessita di calibrazioni quotidiane su sangue capillare, a differenza dei sensori CGM attualmente in uso, e presenta dati di accuratezza in linea con i sensori disponibili. Si tratta di un dispositivo tuttavia non fornito di allarmi e che non si collega con pompe da infusione di insulina. ${ }^{23}$

Dopo che alcuni studi clinici randomizzati ${ }^{33-35}$ hanno analizzato l'efficacia clinica del FGM, evidenziando una riduzione della $\mathrm{HbAlc}$ e del tempo trascorso in ipoglicemia, l'ente regolatorio FDA ha approvato una variante dell'FGM per un utilizzo sostitutivo del SMBG.

\section{La terapia con microinfusore oggi}

La terapia insulinica sottocutanea continua con microinfusore (Continuous Subcutaneous Insulin Infusion, CSII) è il gold standard della terapia insulinica intensiva, sia essa associata a un sistema di monitoraggio in continuo del glucosio (real time o intermittente), sia in combinazione con il solo monitoraggio puntiforme della glicemia capillare ${ }^{36}$ Rispetto alla multiniettiva, la terapia insulinica con pompa mima più fisiologicamente la secrezione insulinica pancreatica e permette aggiustamenti più accurati della dose.

I potenziali benefici della terapia con CSII sono: un miglior controllo dei livelli glicemici ( $\mathrm{HbA1c}$ ), una riduzione della variabilità glicemica (tempo in target), una minor frequenza di ipoglicemie severe, una riduzione del fabbisogno insulinico giornaliero e un miglioramento della qualità della vita grazie soprattutto alla riduzione della paura di ipoglicemie e a una maggiore flessibilità nello stile di vita. ${ }^{37,38}$
Recenti dati sembrano indicare un vantaggio della terapia con CSII rispetto alla multiiniettiva sia in termini di sviluppo delle complicanze, sia di mortalità. ${ }^{18,39-44}$

Le due principali indicazioni cliniche all'utilizzo della CSII sono il mancato raggiungimento del target glicemico con la terapia multiniettiva ottimizzata e/o l'ipoglicemia problematica e/o la perdita della sensibilità all'ipoglicemia. Recenti evidenze suggeriscono l'utilizzo della terapia con CSII anche per il paziente con diabete tipo 2 non in buon controllo nonostante la terapia multiniettiva ottimizzata. ${ }^{36}$

Nonostante l'evoluzione tecnologica degli ultimi anni, il successo della terapia con microinfusore è ancora strettamente dipendente dalle abilità e competenze messe in campo dalla persona che lo utilizza. L'educazione terapeutica per le persone candidate all'utilizzo del CSII, o che già lo utilizzino, è perciò l'elemento indispensabile per l'efficacia di questa terapia. La formazione del paziente non è semplicemente un addestramento, ma un processo continuo di educazione/rinforzo/verifica che deve essere gestito solo da personale dedicato e specializzato. ${ }^{36}$

Negli ultimi anni la tecnologia ha apportato notevoli migliorie agli strumenti per la CSII: i microinfusori di insulina attualmente in commercio posseggono caratteristiche, funzioni ed elementi, programmabili ed eterogenei, alcuni di comprovata utilità, altri meno utilizzati. Tale eterogeneità rende conto dell'importanza di una scelta oculata dello strumento più adatto per ogni paziente. Perciò, se e innegabile che un'adeguata selezione e la preparazione del paziente candidato, sono gli elementi cardine della terapia con CSII, anche la scelta dello strumento può essere rilevante nel determinarne il successo. Tale scelta va discussa e condivisa con il paziente, valutando tra le diverse opzioni quella più appropriata per ogni situazione e, possibilmente, con il miglior rapporto costo/efficacia. Certamente i maggiori ostacoli alla diffusione della terapia insulinica con microinfusore sono determinati dai costi e dall'impegno richiesto alla struttura specialistica in termini di tempo e personale dedicato. ${ }^{36}$

\section{Tecniche di iniezioni dell'insulina}

Negli ultimi tempi è aumentata la consapevolezza dell'importanza di conoscere gli aspetti tecnici per la somministrazione d'insulina nelle persone con diabete mellito, potendo questi influenzare l'ottimizzazione della terapia e il compenso metabolico.

La Tabella 2 riassume le raccomandazioni che scaturiscono dalle conclusioni del workshop Forum for Injection Techniques and Therapy: Expert Recommendations (FITTER) 2015.45

È fondamentale un corretto, completo e strutturato approccio educativo fin dal primo momento in cui il 
Tabella 2. Raccomandazioni per una corretta somministrazione di insulina. ${ }^{45}$

1. Una corretta tecnica d'iniezione è essenziale per garantire un'ottimale azione dell'insulina e degli altri farmaci iniettabili per la cura del diabete. La corretta tecnica iniettiva prevede la scelta dell'ago, la rotazione delle/nelle sedi d'iniezione, la manipolazione e la conservazione dell'insulina, la procedura con cui si inserisce l'ago nella cute, la durata dell'iniezione utilizzando le penne, la manipolazione della cute prima e dopo l'iniezione

(Livello della prova 3, Forza della raccomandazione B)

2. L'insulina deve essere iniettata nel tessuto sottocutaneo integro, evitando il derma, il muscolo, zone cicatriziali, lipoipertrofiche e lipoatrofiche. L'iniezione intramuscolare può comportare un assorbimento più rapido dell'insulina e un potenziale rischio di ipoglicemia, per questo va evitata scrupolosamente soprattutto con gli analoghi lenti dell'insulina

(Livello della prova 2, Forza della raccomandazione B)

3. L'azione dell'insulina non è influenzata dalla profondità con cui viene iniettata nell'ambito del tessuto sottocutaneo

(Livello della prova I, Forza della raccomandazione B)

4. I siti di iniezione raccomandati sono: addome, cosce, glutei e braccia (vedere ALLEGATO 1)

(Livello della prova 1, Forza della raccomandazione $\mathrm{A}$ )

5. L'iniezione con la penna e l'uso di un ago $4 \mathrm{~mm}$ x $32 \mathrm{G}$ inserito perpendicolarmente alla superficie della pelle, garantisce un assorbimento ottimale dell'insulina in tutti i pazienti in terapia insulinica, compresi quelli obesi, è più facile da praticare e da insegnare, nella maggior parte dei casi non necessita della tecnica del pizzicotto (o del pizzico o della plica), causa minore ansia e dolore, comportando migliore accettazione ed aderenza alla terapia.

(Livello della prova 1, Forza della raccomandazione $\mathbf{A}$ )

6. Al fine di minimizzare inavvertite iniezioni intramuscolari, che possono provocare fenomeni di variabilità glicemica, in bambini nella fascia di età $<6$ anni e in pazienti adulti molto magri l'ago da $4 \mathrm{~mm}$ x $32 \mathrm{G}$ dovrebbe essere inserito perpendicolarmente in una plica cutanea. Gli altri pazienti possono iniettare senza la plica cutanea

(Livello della prova 1, Forza della raccomandazione $\mathrm{A}$ )

7. Anche per i pazienti obesi la prima scelta è il $4 \mathrm{~mm} \times 32 \mathrm{G}$, tuttavia l'ago $5 \mathrm{~mm}$ x $31 \mathrm{G}$ può comunque essere accettato

(Livello della prova 1, Forza della raccomandazione $\mathrm{A}$ )

8. Le pazienti insulino-trattate in gravidanza o con diabete gestazionale dovrebbero usare l'ago $4 \mathrm{~mm} \times 32 \mathrm{G}$ come prima scelta, visto l'assottigliarsi dello strato adiposo addominale dovuto all'espansione dell'utero

(Livello della prova 2, Forza della raccomandazione B)

9. Pazienti affetti da tremori o altri disturbi che li rendono incapaci di mantenere in loco il $4 \mathrm{~mm} \times 32 \mathrm{G}$, potrebbero aver bisogno di aghi più lunghi

(Livello della prova 3, Forza della raccomandazione B)

10. Nel caso di iniezione di insulina praticata con siringa, al fine di minimizzare iniezioni intramuscolari inavverite, che possono provocare fenomeni di variabilità glicemica, scegliere il dispositivo con l'ago più corto a disposizione (nel mercato italiano $8 \mathrm{~mm}$ ) applicando la tecnica del pizzico/plica, che va mantenuta fino al termine dell'iniezione e all'estrazione dell' ago dalla cute. Non aspirare insulina da penne o cartucce specie per le U-200 e U-300.

(Livello di Prova 1, Forza della raccomandazione A)

11. Gli analoghi ad azione rapida dell'insulina e gli analoghi basali possono essere iniettati in corrispondenza di qualunque sito, perché il loro assorbimento non è sito-dipendente. Invece, l'insulina umana regolare va preferenzialmente iniettata in corrispondenza della superficie addominale, perché in tale sede il suo assorbimento è più rapido e costante

(Livello di prova 1, Forza della raccomandazione $\mathrm{A}$ )

12. La mancata rotazione delle sedi di iniezione può provocare la formazione di aree di lipodistrofia

(Livello della prova 1, Forza della raccomandazione A)

13. Esiste una correlazione tra il riutilizzo dello stesso ago e la formazione di aree di lipoipertrofia. Il riutilizzo è inoltre associato al dolore e al sanguinamento del sito di iniezione. Pertanto il team diabetologico dovrebbe vietare questa partica scorretta

(Livello della prova 2, Forza della raccomandazione A)

14. L'iniezione d'insulina in aree lipoipertrofiche ne modifica la farmacocinetica e la farmacodinamica, provocando l'assorbimento variabile ed imprevedibile del farmaco e peggiorando il compenso glicemico

(Livello della prova 1, Forza della raccomandazione A)

15. Al fine di evitare fenomeni di lipoipertrofia, il team diabetologico dovrebbe suggerire al paziente uno schema di rotazione facile da seguire sin dall'inizio della terapia insulinica e dovrebbe rivedere col paziente tale schema almeno una volta l'anno

(Livello della prova 2, Forza della raccomandazione A)

16. La corretta rotazione comporta di utilizzare il maggior numero di siti d'iniezione raccomandati (addome, cosce, glutei e braccia), di distanziare ogni iniezione di almeno $1 \mathrm{~cm}$ dalla precedente e di non utilizzare lo stesso punto prima di 4 settimane

(Livello della prova 2, Forza della raccomandazione A)

17. L'ispezione e la palpazione delle sedi di iniezione dei pazienti in terapia iniettiva vanno effettuate almeno una volta l'anno e ad ogni visita se la lipoipertrofia è già presente. L'uso di un gel lubrificante ne facilita l'operazione 
paziente inizia la terapia insulinica, come altrettanto importante è il controllo delle conoscenze e delle abilità dal paziente e la verifica periodica dei siti di iniezione di tutti i pazienti insulino-trattati.

Per un assorbimento ottimale l'insulina deve essere iniettata nel tessuto sottocutaneo e non nel derma o nel muscolo; pertanto la scelta della lunghezza dell'ago è cruciale.

In sintesi, i punti chiave del documento ribadiscono l'importanza di: i) usare aghi corti, sottili e con flusso adeguato; ii) attuare una corretta tecnica iniettiva; iii) evitare l'iniezione intra-muscolare; iv) ruotare costantemente sito di iniezione; v) evitare l'iniezione in aree lipodistrofiche; vi) valutare le potenziali barriere psicologiche prima di iniziare terapia insulinica; vii) smaltire correttamente aghi e lancette; viii) osservare tutte le procedure raccomandate per evitare punture accidentali in tutti i setting assistenziali.

La Tabella 3 definisce le raccomandazioni per la terapia insulinica in ambiente protetto. ${ }^{45,46}$ Viene ribadito l'uso strettamente individuale delle penne, per la documentata aspirazione di materiale biologico nella cartuccia d'insulina. Infatti, nell'uso della penna, quando a fine iniezione cessa la pressione sul pistone, si determina un meccanismo di micro-aspirazione: la quantità di materiale biologico aspirato è di entità più che sufficiente alla trasmissione di numerosi agenti patogeni, oltre ai più noti virus $\mathrm{HIV}, \mathrm{HBV}$ e $\mathrm{HCV}^{47}$

L'utilizzo di pungenti (aghi e lancette pungidito) rimane di competenza del personale sanitario. Solamente ai pazienti non critici ed esperti nell'autosomministrazione di insulina e nell'autocontrollo glicemico, può essere consentito in ambiente ospedaliero l'uso diretto dei pungenti, concordandone le modalità con l'équipe, definendo anche le modalità del corretto smaltimento del materiale utilizzato.

Poiché è raccomandato l'utilizzo di ciascun ago da penna una sola volta, ne è necessaria la rimozione dopo l'uso. Per diminuire il rischio di punture accidentali è vietato lo svitamento di un ago tradizionale non protetto e/o il re-incappucciamento dello stesso. Se in un reparto di degenza sono presenti o vengono utilizzate penne da insulina, devono essere utilizzati aghi con dispositivo di sicurezza, in grado di proteggere contro punture accidentali; la sicurezza deve essere garantita sia dal lato paziente (punta dell'ago) sia per quello di inserzione nella cartuccia (punta-cartuccia). È stato calcolato che circa il 10\% delle punture accidentali con aghi per penna avviene proprio con la punta-cartuccia. Viene inoltre consigliato di utilizzare

Tabella 2. Segue dalla pagina precedente

\section{(Livello della prova 2, Forza della raccomandazione $\mathbf{A}$ )}

18. Il team diabetologico deve monitorare e registrare l'evoluzione delle lipoipertrofie, utilizzando fotografie e/o moduli graduati che rappresentano le sedi di iniezione per descriverne la misura, la forma e la consistenza

\section{(Livello della prova 2, Forza della raccomandazione A)}

19. Nel passaggio dell'iniezione da una zona lipodistrofica ad una zona sana, le dosi di insulina andrebbero monitorate e/o ridotte perché l'assorbimento potrebbe risultare migliorato. La riduzione della dose insulinica varia da individuo ad individuo e deve essere guidata da un'intensificazione dell'automonitoraggio glicemico

(Livello della prova 2, Foza della raccomandazione A)

20. Per un'efficace azione educativa il team diabetologico deve affrontare col paziente gli ARGOMENTI ESSENZIALI (vedere ALLEGATO 5), sia verbalmente che in forma scritta e deve assicurarsi che tutte le informazioni siano state perfettamente comprese. Sono fondamentali dimostrazioni pratiche su come fare correttamente l'iniezione, in cui il paziente mostra praticamente quanto ha appreso. Per la cronicità della malattia l'azione educativa va attuata con modalità strutturata sia all'inizio della terapia iniettiva stessa che successivamente, almeno una volta l'anno. L'azione educativa non può prescindere da una valutazione di aderenza alla cura, verificando in concreto la corrispondenza tra aghi prescritti e aghi usati, il tipo di penne utilizzate e tutti i dispositivi dispensati dal farmacista

(Livello di Prova 1, Forza della raccomandazione A)

21. Oltre ad ispezionare periodicamente tutte le sedi d'iniezione, bisogna coinvolgere il paziente sulla sua esperienza iniettiva: interrogarlo sull'eventuale disagio/dolore, sull'eventuale comparsa di sanguinamenti o lividi o sulla perdita di insulina dal sito iniettivo, rassicurandolo e proponendogli possibili soluzioni

(Livello di Prova 3, Forza della raccomandazione A)

22. Sensibilizzare il paziente e i caregiver sulle punture accidentali. In nessuna circostanza i rifiuti taglienti/pungenti devono finire nella normale spazzatura e/o tra i normali rifiuti pubblici

(Livello di Prova 1, Forza della raccomandazione A)

23. L'uso domiciliare di aghi o siringhe di sicurezza va previsto anche per speciali popolazioni di persone in terapia iniettiva, portatori di infezioni da HIV, HBV e HCV

(Livello di Prova 2, Forza della raccomandazione B)

24. L'insulina d'uso quotidiano va tenuta a temperatura ambiente, lontana da fonti di calore o esposta a luce solare diretta per circa 28-30 giorni, seguendo le indicazioni del produttore, approvate da AIFA

(Livello di Prova 2, Forza della raccomandazione B) 
Tabella 3. Raccomandazioni per una corretta somministrazione di insulina in ambiente protetto. ${ }^{45}$

24. In accordo con la Direttiva Europea 2010 e con le norme da essa derivanti adottate negli stati membri, tutte le pratiche iniettive o altre azioni eseguite per la gestione del diabete da personale sanitario in ambienti in cui si presta assistenza (ospedali, aree di emergenza, ambulatori, ambulanze, etc.) vanno praticate esclusivamente con dispositivi di sicurezza, per minimizzare il rischio di puntura accidentale e per garantire la tutela della salute degli operatori, dei pazienti e dei loro familiari in tutte le fasi di utilizzo, fino allo smaltimento dei pungenti

(Livello di Prova I, Forza della raccomandazione A)

25. Gli aghi per le penne da insulina devono essere dotati di dispositivo di sicurezza in grado di proteggere contro punture accidentali, sia anteriormente (lato paziente) che posteriormente (lato cartuccia). Aghi per penna da insulina dotati di dispositivo di sicurezza solo anteriormente (lato paziente) e privo di analogo dispositivo di sicurezza dal lato posteriore (lato cartuccia) non garantiscono adeguata protezione dal rischio di puntura accidentale

(Livello di Prova 2, Forza della raccomandazione A)

26. Il reincappucciamento degli aghi e delle siringhe è una manovra proibita così come la manovra di portare la mano davanti al tagliente. Quindi anche lo svitamento dell'ago dalla penna da insulina senza protezione è proibito in ambiente protetto.

(Livello di Prova 1, Forza della raccomandazione A)

27. Per minimizzare il rischio di punture accidentali dovute alla plica cutanea, utilizzare aghi corti senza sollevare la plica (4 e 5 mm)

(Livello di Prova 1, Forza della raccomandazione A)

28. In tutti gli ambienti sanitari in cui si utilizzano penne per insulina, devono essere seguite procedure rigorose secondo cui ad ogni paziente deve corrispondere un'unica penna da insulina, per evitare il rischio di trasmissione di infezioni tra diversi pazienti per l'uso della stessa penna pre-riempita. Ciascuna penna va personalizzata con i dati della persona per cui viene utilizzata e con la data di inizio d'uso

(Livello di Prova 1, Forza della raccomandazione A)

29. Non è raccomandabile aspirare insulina dalla cartuccia della penna con una siringa

(Livello di Prova II, Forza della raccomandazione A)

30. La conservazione dell'insulina in ospedale e a domicilio (penne e flaconi) deve rispettare le indicazioni dei produttori fornite dalle schede tecniche approvate dall'AIFA. Tali indicazioni devono essere oggetto di educazione per i pazienti

(Livello di Prova 2, Forza della raccomandazione B)

aghi corti (4 e $5 \mathrm{~mm}$ ), senza sollevare la plica, per minimizzare il rischio di punture accidentali dovute alla plica cutanea.

Si ribadisce che non è raccomandabile aspirare insulina dalla cartuccia della penna con una siringa, in quanto non esistono prove che questa pratica garantisca il prelievo di dosi corrette di farmaco e perché i due sistemi iniettivi sono stati progettati per un uso diverso da questa procedura. Inoltre, il prelievo d'insulina da una cartuccia o da una penna con una siringa può determinare la formazione di bolle d'aria e può pertanto verificarsi un errore di prelievo della dose successiva se la penna viene riutilizzata, con evidenti importanti ricadute cliniche.

L'infusione continua di insulina mediante ago e set di infusione, espone il tessuto sottocutaneo all'azione di tipo reservoir dell'insulina nel medesimo sito e per tutto il tempo in cui lo stesso set di infusione vi permane, prima di essere sostituito e posizionato in altra sede. È quindi cruciale il rispetto dei tempi e delle sedi di applicazione del set infusionale, come è cruciale la personalizzazione del tipo di ago per lunghezza e angolazione. Infine, l'uso del microinfusore non evita le complicanze locali della terapia multi-iniettiva, lipoipertofie e lipoatrofie. Ne consegue che anche per que- sti pazienti è cruciale seguire la corretta tecnica di rotazione delle sedi di iniezione.

\section{Bibliografia}

1. Cryer PE. Hypoglycemia in diabetes: pathophysiology, prevalence and prevention. Alexandria, Virginia: American Diabetes Association; 2009. pp 9.

2. Weinstock RS, DuBose SN, Bergenstal RM, et al. T1D exchange severe hypoglycemia in older adults with type 1 diabetes study groups. risk factors associated with severe hypoglycemia in older adults with type 1 diabetes. Diabetes Care 2016;39:603-10.

3. The DCCT Research Group. Epidemiology of severe hypoglycemia in the diabetes control and complications trial. Am J Med 1991;90:450-9.

4. UK Prospective Diabetes Study (UKPDS) Group. Intensive blood-glucose control with sulphonylureas or insulin compared with conventional treatment and risk of complications in patients with type 2 diabetes (UKPDS 33). Lancet 1988;352:837-53.

5. Zoungas S1, Patel A, Chalmers J, et al. (ADVANCE Collaborative Group). Severe hypoglycemia and risks of vascular events and death. N Engl J Med 2010;363:1410-8.

6. Miller ME, Bonds DE, Gerstein HC, et al. (ACCORD Investigators). The effects of baseline characteristics, 
glycaemia treatment approach, and glycated haemoglobin concentration on the risk of severe hypoglycaemia: post hoc epidemiological analysis of the ACCORD study. BMJ 2010;340:b5444.

7. Johnston SS, Conner C, Aagren M, et al. Evidence linking hypoglycemic events to an increased risk of acute cardiovascular events in patients with type 2 diabetes. Diabetes Care 2011;34:1164-70.

8. Desouza CV, Bolli GB, Fonseca V. Hypoglycemia, diabetes, and cardiovascular events. Diabetes Care 2010;33:1389-94.

9. Whitmer RA, Karter AJ, Yaffe K, et al. Hypoglycemic episodes and risk of dementia in older patients with type 2 diabetes mellitus. JAMA 2009;301:1565-72.

10. Johnston SS, Conner C, Aagren M, et al. Association between hypoglycaemic events and fall-related fractures in Medicare-covered patients with type 2 diabetes. Diabetes Obes Metab 2012;14:634-43.

11. McCoy RG, Van Houten HK, Ziegenfuss JY, et al. Increased mortality of patients with diabetes reporting severe hypoglycemia. Diabetes Care 2012;35:1897-901.

12. Green AJ, Fox KM, Grandy S (SHIELD Study Group). Self-reported hypoglycemia and impact on quality of life and depression among adults with type 2 diabetes mellitus. Diabetes Res Clin Pract 2012;96:313-8.

13. Laiteerapong N, Karter AJ, Liu JY, et al. Correlates of quality of life in older adults with diabetes: the diabetes and aging study. Diabetes Care 2011;34:1749-53.

14. Leiter LA, Yale J-F, Chiasson JL, et al. Assessment of the impact of fear of hypoglycemic episodes on glycemic and hypoglycemia management. Canad J Diabet 2005;29:186-92.

15. Marinelli Andreoli A, Chiovoloni M, et al. Ricoveri per ipoglicemia grave in soggetti con diabete mellito di tipo 2: impatto clinico e costi sanitari. G It Diabetol Metab 2015;35:130-5.

16. Marchesini G, Veronese G, Forlani G, et al. Italian Society of Emergency Medicine (SIMEU). The management of severe hypoglycemia by the emergency system: the HYPOTHESYS study. Nutr Metab Cardiovasc Dis 2014;24:1181-8.

17. Osservatorio ARNO Diabete. Il profilo assistenziale della popolazione con diabete. CINECA SID Rapporto 2015. Disponibile su: http://www.siditalia.it/news/84419-11-2015 Ultima consultazione on line 31/01/2019.

18. AMD-SID. Standard di Cura del Diabete - aprile 2018. Ultima consultazione on line 25/01/2019.

19. Ceriello A, Armentano V, De Micheli A, et al. La personalizzazione della terapia: innovazione nella gestione del paziente con diabete tipo 2. Giorn AMD 2011;14:35-45.

20. AMD-SID. Standard italiani per la cura del diabete mellito 2014. Disponibile su: http://www.standarditaliani. it/.29

21. Monami M, Luzzi C, Lamanna C, et al. Threeyear mortality in diabetic patients treated with different combinations of insulin secretagogues and metformin. Diabetes Metab Res Rev 2006;22:477-82.

22. Ministero della Salute. DG Programmazione Sanitaria Commissione 22 Nazionale Diabete. Ultima consultazione on line 25/01/2019. Disponibile su: http:// www.salute.gov.it/imgs/C_17_pubblicazioni_1885_allegato.pdf

23. Pintaudi B. Gli Standard Italiani 2018 per la terapia del diabete mellito. The 2018 Italian Standards for the treatment of diabetes mellitus. Ultima consultazione in web 20/01/20019.

24. Aleppo G, Ruedy KJ, Riddlesworth TD, et al. REPLACE-BG: A randomized trial comparing continuous glucose monitoring with and without routine blood glucose monitoring in well-controlled adults with type $1 \mathrm{di}$ abetes. Diabetes Care 2017;40:38-45.

25. Food and Drug Administration. Approval Order: Dexcom G5 Mobile Continuous Glucose Monitoring System. PI20005. Silver Spring, MD: Department of Health and Human Services; 2016.

26. Pickup JC, Freeman SC, Sutton AJ. Glycaemic control in type 1 diabetes during real time continuous glucose monitoring compared with self monitoring of blood glucose: meta-analysis of randomised controlled trials using individual patient data. BMJ 2011;343.

27. Yeh HC, Brown TT, Maruthur N, et al. Comparative effectiveness and safety of methods of insulin delivery and glucose monitoring for diabetes mellitus: a systematic review and meta-analysis. Ann Intern Med 2012; 157:336-47.

28. Battelino T, Phillip M, Bratina N, et al. Effect of continuous glucose monitoring on hypoglycemia in type 1 diabetes. Diabetes Care. 2011; 34: 795-800.

29. Battelino T, Conget I, Olsen B, et al. (SWITCH Study Group). The use and efficacy of continuous glucose monitoring in type 1 diabetes treated with insulin pump therapy: a randomized controlled trial. Diabetologia 2012;55:3155-62.

30. New JP, Ajjan R, Pfeiffer AF, et al. Continuous glucose monitoring in people with diabetes: the randomized controlled Glucose Level Awareness in Diabetes Study (GLADIS). Diabet Med 2015;32:609-17.

31. van Beers CA, DeVries JH, Kleijer SJ, et al. Continuous glucose monitoring for patients with type 1 diabetes and impaired awareness of hypoglycaemia (IN CONTROL): a randomised, open-label, crossover trial. Lancet Diabetes Endocrinol 2016;4:893-902.

32. Heinemann L, Freckmann G, Ehrmann D, et al. Realtime continuous glucose monitoring in adults with type 1 diabetes and impaired hypoglycaemia awareness or severe hypoglycaemia treated with multiple daily insulin injections (HypoDE): a multicentre, randomised controlled trial. Lancet 2018;391:1367-77.

33. Haak T, Hanaire H, Ajjan R, et al. G flash glucose-sensing technology as a replacement for blood glucose monitoring for the management of insulin-treated type 2 diabetes: a multicenter, open-label randomized controlled trial. Diabetes Ther 2017;8:55-73.

34. Haak T, Hanaire H, et al. Use of flash glucose-sensing technology for 12 months as a replacement for blood glucose monitoring in insulin-treated type 2 diabetes. Diabetes Ther 2017;8:573-86.

35. Bolinder J, Antuna R, Geelhoed-Duijvestijn P, et al. Novel glucose-sensing technology and hypoglycaemia in type 1 diabetes: a multicentre, non-masked, randomized controlled trial. Lancet 2016;388:2254-63.

36. Girelli A, Di Bartolo P. La terapia con microinfusore oggi: cosa considerare per una scelta appropriata dello strumento? Media 2018;18:102-8.

37. Cummins E, Royle P, Snaith A, et al. Clinical effectiveness and costeffectiveness of continuous subcutaneous insulin 
infusion for diabetes: systematic review and economic evaluation. Health Technol Assess 2010;14:1-181.

38. Nicolucci A, Maione A, Franciosi M, et al. EQuality1 Study Group-Evaluation of QUALITY of Life and Costs in Diabetes Type 1, Quality of life and treatment satisfaction in adults with Type 1 diabetes: a comparison between continuous subcutaneous insulin infusion and multiple daily injections. Diabet Med 2008;25:213-20.

39. Lepore G, Bruttomesso D, Bonomo M, et al. Continuous subcutaneous insulin infusion is more effective than multiple daily insulin injections in preventing albumin excretion rate increase in Type 1 diabetic patients. Diabet Med 2009;26:602-8.

40. Steineck I, Cederholm J, Eliasson B, et al.; Swedish National Diabetes Register. Insulin pump therapy, multiple daily injections, and cardiovascular mortality in 18168 people with type 1 diabetes: observational study. BMJ 2015;350:h3234.

41. American Diabetes Association. Standards of Medical Care in Diabetes - 2018. Diabetes Care 2018;41:S15159.

42. Reznik Y, Cohen O, Aronson R, et al. OpT2mise Study Group. Insulin pump treatment compared with multiple daily injections for treatment of type 2 diabetes (OpT2mise): a randomized open-label controlled trial. Lancet 2014;384:1265-72.
43. Pickup JC, Reznik Y, Sutton AJ. Glycemic control during continuous subcutaneous insulin infusion versus multiple daily insulin injections in type 2 diabetes: individual patient data meta-analysis and meta-regression of randomized controlled trials. Diabetes Care 2017;40:715-22.

44. Meade LT, Rushton WE. Optimizing insulin pump therapy: a quality improvement project. Diabetes Educ 2013;39:841-7.

45. Documento di Consenso sul Recepimento Italiano del Forum for Injection Technique and Therapy Expert Recommendations 2015 Aggiornamento e integrazioni a cura del gruppo intersocietario AMD-OSDI sulle teniche iniettive 16.05.2017. Ultima consultazione on line 25/01/2019.

46. Frid AH, Kreugel G, Grassi G, et al. New insulin delivery recommendations. Mayo Clin Proc 2016;91:1231-55.

47. Gentile S, Strollo F, De Rosa N, et al. Injection-related local side effects in the treatment of diabetes mellitus: a methodological approach and possible solutions. Consensus Statement of AMD-OSDI Study Group on Injection Technique. Diabetic Complications, Creative Commons, November 01, 2016. Disponibile su: http:// www.smgebooks.com/diabetic-complications/chapters/DC-16-04.pdf 


\title{
Strumenti per una gestione sicura delle allergie a farmaci e delle interazioni farmacologiche
}

\author{
Tiziana Marcella Attardo
}

Direzione Medica, Diabetologia e Malattie del Metabolismo, UO di Medicina Interna, Ospedale Barone Lombardo, Canicattì (AG), Italia

\section{Introduzione}

L'Organizzazione Mondiale della Sanità (OMS) definisce adverse drug reactions (ADR) tutte le conseguenze non terapeutiche di un farmaco, ad eccezione dei fallimenti terapeutici, degli avvelenamenti intenzionali o accidentali, degli errori di somministrazione e dell'abuso. ${ }^{1}$

Le ADR dovrebbero essere differenziate dagli eventi avversi da farmaci $(\mathrm{ADE})^{2}$ in quanto le ADE si caratterizzano per comprendere, oltre alle ADR, i danni correlati agli errori terapeutici e alle interazioni farmaco/cibo.

Le ADR si distinguono in prevedibili e non prevedibili. ${ }^{3,4}$

Quelle prevedibili o di tipo A (augmented) rappresentano circa l' $80 \%$ delle ADR, sono dose dipendenti, correlate all'azione farmacologica e si verificano in soggetti normali.

Possono presentarsi come: i) effetti collaterali: strettamente correlati all'azione farmacologica principale. Il loro manifestarsi dipende dalla grande variabilità della tolleranza individuale (per esempio la sonnolenza da antistaminici o il sanguinamento gastrointestinale da FANS); ii) effetti secondari: conseguenza indiretta della principale azione farmacologica (esempio: candidosi da antibiotici o corticosteroidi); iii) da sovradosaggio o concentrazione eccessiva del farmaco a livello recettoriale (esempi: morfina in presenza di epatopatie, aminoglicosidici in caso di insuf-

Corrispondente: Tiziana Marcella Attardo, Direzione Medica, Diabetologia e Malattie del Metabolismo, UO di Medicina Interna, Ospedale Barone Lombardo, Canicattì (AG), Italia.

E-mail: claudia.fantucchio@alice.it

Articolo pubblicato secondo la Creative Commons Attribution NonCommercial 4.0 License (CC BY-NC 4.0).

${ }^{\circ}$ Copyright: the Author(s), 2019

Licensee PAGEPress, Italy

QUADERNI - Italian Journal of Medicine 2019; 7(6):145-150 ficienza renale); iv) da interazioni farmacologiche, cioè la modificazione dell'effetto del farmaco dovute all'uso recente o contemporaneo di un altro farmaco $o$ di più farmaci (interazioni farmaco-farmaco), o all'ingestione di cibo (interazioni farmaco-cibo) o all'ingestione di integratori dietetici (interazioni farmaco-integratori), con la conseguenza di ottenere o un aumento o una diminuzione degli effetti di uno o d'entrambi i farmaci; v) correlazioni specifiche farmaco/malattia (esempio: rash da ampicillina in corso di mononucleosi infettiva).

Quelle non prevedibili, o di tipo $B$ o bizzarre sono dose indipendenti, non correlate all'azione farmacologica e si verificano in soggetti predisposti: i) da idiosincrasia: da difetti metabolici o deficienze enzimatiche geneticamente determinati, non manifesti in condizioni normali, ma che si evidenziano solo dopo l'assunzione di particolari farmaci (esempio: emolisi da primachina e altri farmaci ossidanti in soggetti con deficit di G6PDH); ii) da intolleranza: da abbassamento della soglia alla normale azione farmacologica del farmaco con un effetto quantitativamente aumentato, ma qualitativamente normale (esempio: tinnitus da chinino o salicilati); iii) da ipersensibilità: allergiche (mediate da meccanismi immunologici noti) e non allergiche (mimano le reazioni IgE-mediate). ${ }^{4}$

Le ADR rappresentano dal 3 al 6\% di tutti i ricoveri ospedalieri e si verificano nel $10-15 \%$ dei pazienti ospedalizzati; esse correlano sfavorevolmente con morbilità, prolungamento dell'ospedalizzazione, mortalità e, di conseguenza, aumento dei costi. ${ }^{3}$ Si calcola che nei paesi occidentali il 10-15\% dei pazienti ospedalizzati vada incontro a reazioni a farmaci per il $6,7 \%$ gravi o mortali.

Le ADR sono una patologia di importante impatto socio-economico ed in costante crescita in tutti i paesi del mondo, anche in relazione al sempre maggior consumo di farmaci. Da qui la necessità di una gestione appropriata dell'evento ADR al fine di evidenziarne le precisi e peculiari circostanze e, soprattutto, di impedire che l'evento possa ripresentarsi.

In caso di ADR l'anamnesi deve essere:-7 ${ }^{4}$ ) esau- 
riente: può essere necessario un colloquio anche di 1 ora!; ii) dettagliata: è utile collaborare con il Medico di Medicina Generale e se la reazione è avvenuta in età infantile è indispensabile parlare con i genitori; iii) documentata: raccogliere tutti i documenti utili a identificare i farmaci somministrati in occasione di una sospetta ADR: fotocopie di cartelle cliniche, fotocopie di cartelle anestesiologiche, referti del pronto soccorso, libretti sanitari, libretti delle vaccinazioni, referti di prove allergologiche, etc; iv) completa: è molto utile impiegare un questionario; ogni specialista o centro può elaborarne uno ad hoc o adottare quello pubblicato dall'ENDA (European Network of Drug Allergy), il gruppo d'interesse sulle reazioni avverse ai farmaci dell'EAACI (European Academy of Allergy and Clinical Immunology), tradotto anche in italiano; ${ }^{8}$ v) estesa anche alle più comuni manifestazioni allergiche (asma, rinocongiuntivite, orticaria angioedema, eczema), eventualmente anche autoimmuni e comprensiva delle più importanti patologie a causa delle quali il paziente ha assunto o dovrà assumere farmaci ${ }^{4}$

Non bisogna dimenticare, infine, che possono essere causa di allergia non solo i farmaci di sintesi, ma anche quelli naturali a base di erbe (fitoterapici). Le reazioni ai fitoterapici sono spesso insidiose, perché più difficili da identificare, e possono essere provocate da erbe esotiche usate in tipi di medicina tradizionale poco noti in paesi come il nostro. Pertanto nella raccolta anamnestica è molto importante indagare a $360^{\circ}$ sull'assunzione anche di sostanze da banco, di farmaci omeopatici e sui prodotti naturali.

Si ricorda che la segnalazione dell'ADR alla rete nazionale di farmacovigilanza è obbligatoria e deve avvenire mediante una specifica scheda disponibile sul sito dell'Agenzia Italiana del Farmaco. ${ }^{9-16}$ La scheda compilata deve essere inviata al responsabile aziendale per la farmacovigilanza.

\section{Strumenti e raccomandazioni}

1. Non trascurare strumenti come la ricognizione e riconciliazione farmacologia: i) conoscere tutti i farmaci assunti dal paziente, compresi quelli prescritti da altri colleghi, come anche tutti i farmaci da banco, prodotti a base di erbe e integratori nutrizionali; ii) chiedere ai pazienti informazioni rilevanti sul consumo d'alcol e sulla dieta; iii) cercare di prescrivere il minor numero di farmaci possibile, alla dose più bassa possibile e per il minor tempo possibile; iv) conoscere gli effetti, desiderati ed indesiderati, di tutti i farmaci utilizzati, perché di solito questi effetti comprendono la gamma delle interazioni farmacologiche; v) se possibile, utilizzare farmaci con un ampio margine di sicurezza in modo che eventuali interazioni impreviste non causino tossicità.
2. Informare il paziente sugli effetti e sui rischi legati alle terapie che sta assumendo. Un paziente ben informato può costituire un'importante fonte di informazioni per il medico stesso.

3. Ricercare sistematicamente gli effetti collaterali interrogando e osservando i pazienti specialmente dopo una variazione della terapia; tenere presente che alcune interazioni (p. es., effetti dovuti all'induzione enzimatica) possono richiedere anche più di una settimana per manifestarsi.

4. Considerare le interazioni farmacologiche come una delle cause possibili di qualunque problema non previsto. ${ }^{5}$

5. Determinare le concentrazioni sieriche dei farmaci assunti - quando possibile ed indicato.

Molto utile è uno strumento presente sul sito Torrinomedica.it, ${ }^{17}$ che permette di verificare in un istante ogni possibile interazione per ogni singolo farmaco come anche di verificare la compatibilità tra due farmaci inseriti nelle caselle di testo. Analoghi siti internazionali che sono di pronta disponibilità sul web, ${ }^{18-20}$ utili anche per valutare in maniera immediata interazioni tra farmaco e cibo, come anche tra farmaco e prodotto da banco, prodotto omeopatico o da erboristeria.

6. Le Dieci Guide (Tabella 1), ${ }^{18}$ elaborate dal gruppo Sicurezza dei Farmaci del Ministero della Salute, sono rivolte a tutti coloro che sono coinvolti, a vari livelli, nella promozione della sicurezza dei pazienti. Scopo delle stesse è fornire indicazioni precise, provenienti dalla letteratura e dalle esperienze nazionali ed internazionali, a cittadini, familiari, volontari, pazienti degli studi odontoiatrici, operatori e aziende sanitarie per concorrere alla sicurezza delle cure. Non si tratta di manuali, né di linee guida per i servizi sanitari, ma di fogli informativi orientati a segnalare accorgimenti per la sicurezza dei pazienti.

7. I Criteri di Beers. La Società Americana di Geriatria (AGS), ha recentemente aggiornato i Beers Criteria, l'elenco dei farmaci potenzialmente inappropriati nella popolazione anziana. ${ }^{12} \mathrm{Si}$ tratta di un supporto agli operatori sanitari per la corretta prescrizione dei medicinali negli over 65 , che si concentra sulla de-prescrizione e conseguentemente sulla possibilità di evitare al paziente anziano possibili reazioni avverse e danni causati da interazioni tra farmaci. Dal 2011, l'AGS è stata l'amministratore dei criteri e ha prodotto aggiornamenti su un ciclo triennale. L'AGS Beers Criteria è un elenco esplicito di farmaci che è preferibile evitare negli anziani nella maggior parte delle circostanze o in situazioni specifiche, come in certe malattie o condizioni. ${ }^{13-16}$

Nel dettaglio la revisione comprende: i) una guida separata sulle 13 combinazioni di medicinali da 
evitare per cause note di interazioni farmaco-farmaco Alcuni trattamenti per diverse patologie comuni negli adulti più anziani possono essere inappropriati quando prescritti nello stesso momento, perché possono aumentare i rischi di altri problemi di salute (cadute e fratture, incontinenza urinaria o tossicità da farmaco); ii) un elenco specifico di prescrizioni da evitare o correggere in base alla funzionalità renale della persona. Una funzione renale compromessa può incidere sul modo in cui il farmaco viene assorbito e metabolizzato. I Criteri 2015 elencano 20 farmaci potenzialmente problematici per gli anziani con insufficienza renale da moderata a severa, in quanto potrebbero associarsi ad aumento di una serie di effetti come nausea, diarrea, sanguinamento, neurotossicità e tossicità midollare; iii) tre nuovi farmaci e due nuove classi di medicinali sono stati aggiunti all'elenco delle avvertenze per la maggior parte delle persone più anziane o per quelle con particolari problemi di salute. Tra questi gli inibitori di pompa protonica, spesso prescritti per le persone affette da reflusso acido o ulcere gastriche. Diversi studi supportano ora un legame tra questi farmaci e un aumento del rischio di perdita di massa ossea, fratture e infezioni batteriche gravi; iv) aggiornamenti per rimuovere diversi farmaci dai criteri di Beers, sia perché non più disponibili sia perché la patologia sottesa o $i$ problemi che tali farmaci affrontano non sono più

Tabella 1. Guida all'utilizzo dei farmaci in sicurezza a cura del Ministero alla Salute. ${ }^{9}$

\section{1 - Assumo i farmaci solo su indicazione del medico}

Uso i farmaci solo se prescritti dal medico e non perché consigliati da un parente o da un amico oppure perché ne ho sentito parlare alla televisione o letto informazioni su riviste o su Internet. Quando assumo farmaci acquistabili senza ricetta chiedo comunque consiglio al medico o al farmacista

\section{2 - Voglio essere informato e consapevole della cura che sto facendo}

Non esito a chiedere al medico o al farmacista tutte le informazioni sui farmaci che devo prendere, inclusi gli effetti collaterali e le eventuali interazioni con altri medicinali, prodotti erboristici e fitoterapici, bevande o alimenti. Se non ho capito bene chiedo di ripetere con parole più semplici

\section{3 - Parlo apertamente con il medico}

Quando vado dal medico porto sempre la lista dei farmaci che sto prendendo (eventualmente con i foglietti illustrativi), anche quelli prescritti da altri medici. Riferisco se sto facendo uso di prodotti omeopatici, erboristici, fitoterapici, integratori o altri preparati acquistati in negozi specializzati; lo informo se sono in allattamento, in stato di gravidanza o se ho in progetto di esserlo

\section{4 - Informo il medico se sono allergico ai farmaci}

Anche durante il ricovero devo SEMPRE riferire ai medici e agli infermieri se sono allergico ad alcuni farmaci o altri prodotti e/o materiali e se ho manifestato intolleranza ad alcuni alimenti

\section{5 - Riferisco al medico i disturbi apparsi durante la cura}

Se durante la cura compaiono disturbi di cui il medico non mi aveva avvertito, lo avviso subito: il medico mi dirà come comportarmi

\section{6 - Rispetto le indicazioni fornite dal medico Farmaco giusto, al paziente giusto, nella dose giusta, nel momento giusto}

Controllo sempre che il farmaco da assumere sia quello prescrittomi e che la quantità sia quella indicata dal medico. Se sbaglio medicina o quantità, prima di prendere iniziative, consulto il medico. Se mi dimentico di prendere un farmaco non ne prendo due successivamente per colmare la dose saltata. Aumento o diminuisco la dose solo quando me lo dice il medico

\section{7 - Presto attenzione se devo assumere più farmaci}

Se mi sono stati prescritti più farmaci per diverse malattie riferisco al medico o al farmacista la difficoltà a ricordare tutti i nomi e gli orari di assunzione ed eventualmente chiedo di scrivere sulle confezioni di ciascun farmaco a cosa serve e l'orario in cui va preso o di farmi un promemoria delle medicine che devo assumere con tutte le indicazioni per me necessarie

\section{8 - Non sostituisco i farmaci né modifico la cura prescritta di mia iniziativa}

Quando sono ricoverato riferisco al medico e all'infermiere quali medicine sto prendendo e, per evitare interazioni, non prendo i farmaci che eventualmente ho portato da casa: è il medico che decide se confermare, sostituire o integrare la cura domiciliare

\section{9 - Conservo i farmaci in modo sicuro}

In casa conservo i farmaci in un luogo asciutto, al riparo dalla luce o, se indicato, in frigorifero e lontano dalla portata dei bambini. Separo i farmaci che hanno la confezione o il nome simili e che possono trarmi in inganno, e separo quelli per i bambini da quelli per gli adulti. Non lascio i blister (gli involucri) o i flaconi aperti a portata di tutti: è buona abitudine conservare i farmaci nelle proprie confezioni originarie con il foglietto illustrativo. Per i colliri, gli sciroppi e le soluzioni in flaconi multidose, è utile annotare sulla confezione la data della prima apertura e la data dell'ultima utilizzazione. Prima di prendere un farmaco controllo la scadenza e la corretta conservazione osservando eventuali cambiamenti di colore e/o odore.Non lascio i farmaci in auto, specie nel periodo estivo o se è esposta al sole. Durante l'estate se sono in viaggio, anche per brevi spostamenti, trasporto i medicinali, che richiedono una determinata temperatura di conservazione, in busta termica e/o con ghiaccio sintetico; se viaggio in aereo porto sempre i farmaci con me

\section{0 - Se assisto qualcuno, non esito a chiedere aiuto}

Se assisto a casa una persona malata, presto attenzione ai farmaci che deve assumere, attenendomi scrupolosamente alle indicazioni fornite dal medico: se ho difficoltà o dubbi devo avvertire subito i familiari dell'assistito, se possibile, o il medico 
limitati alle persone anziane. Sono stati, ad esempio, eliminati farmaci potenzialmente inappropriati per persone con stipsi cronica, perché la costipazione è comune in tutte le fasce d'età e le considerazioni sulle prescrizioni non sono specifiche per gli anziani; v) diverse modifiche alle raccomandazioni sui farmaci potenzialmente inappropriati. Alla luce delle crescenti evidenze sui danni che gli antipsicotici provocano in persone con delirio e demenza senza migliorare i risultati di cura, i Beers Criteria sostengono di evitare antipsicotici per $i$ problemi comportamentali del tutto, a meno che non si sia ottenuta una modifica dei comportamenti o che l'anziano rappresenti una minaccia fisica per sé o per gli altri; vi) infine, il gruppo di esperti ha inoltre ribadito che i farmaci potenzialmente inappropriati sono esattamente potenzialmente inappropriati e quindi meritano una vigilanza speciale, ma non devono essere intesi come universalmente inammissibili in tutti i casi o per tutte le persone. Inoltre i criteri di Beers non dovrebbero mai da soli dettare protocolli di prescrizione, né servire da giustificazione per limitare la copertura sanitaria. Questo strumento funziona meglio come punto di partenza per orientare il regime farmacologico globale di una persona.

8. Principi analoghi ispirano altri strumenti come lo STOPP (Screening Tool of Older Persons' Prescriptions) e START (Screening Tool to Alert to Right Treatment) che oltre alla lista dei farmaci da evitare negli anziani comprende anche la lista dei farmaci da non omettere perchè dotati di benefici dimostrati anche negli anziani. ${ }^{21} \mathrm{~A}$ tal proposito si segnala che l'Istituto di Ricerche Farmacologiche Mario Negri ${ }^{22}$ mette a disposizione gratuitamente online, previa registrazione, un sistema completo che include: i) interazioni tra farmaci (database delle interazioni realizzato ed aggiornato dall'Istituto di Ricerche Farmacologiche Mario Negri IRCCS); ii) farmaci potenzialmente inappropriati nell'anziano secondo differenti criteri delle letteratura scientifica (Beers; START/STOPP); iii) valutazione del carico anticolinergico (Anticholinergic Cognitive Burden scale); iv) modalità di sospensione dei farmaci che necessitano riduzione graduale delle dosi; v) dosaggio dei farmaci in soggetti con alterata funzionalità renale; vi) raccomandazioni di Choosing Wisely-Italy sulla terapia farmacologica; vii) valutazione dei benefici e dei rischi attesi dalla terapia farmacologica attraverso l'impiego degli NNT\&NNH; viii) algoritmo GerontoNet ADR Risk Score, per l'identificazione dei pazienti a maggior rischio di effetti indesiderati da farmaco; ix) algoritmo Naranjo per la valutazione del nesso di causalità tra un farmaco ed un evento avverso; x) algoritmo DIPS (Drug Interaction Probability Scale) per la valutazione del nesso di causalità evento avverso-interazione tra farmaci.

\section{Bibliografia}

1. World Health Organization. International drug monitoring: the role of national centres. World Health Organ Tech Rep Ser 1972;498:1-25.

2. Morimoto T, Gandhi TK, Seger AC, et al. Adverse drug events and medication errors: detection and classification methods. Qual Saf Health Care 2004;13:306-14.

3. Thong BY-H, Tan T-C. Epidemiology and risk factors for drug allergy. Br J Clin Pharmacol 2011;71:684-700.

4. Reazioni avverse ai farmaci - SNLG Regioni - Linee guida per la diagnosi e la cura delle allergopatie. Regione Toscana 2011.

5. www.msdmanuals.com/it-it/professionale/farmacologiaclinica/fattori-che-influenzano-la-risposta-ai-farmaci/interazioni-tra-farmaci Data ultima consultazione on line $03 / 02 / 2019$

6. Johansson SG, Bieber T, Dahl R, et al. Revised nomenclature for allergy for global use: report of the nomenclature review committee of the World Allergy Organization, October 2003. J Allergy Clin Immunol 2004;113:832-6.

7. Rawlins MD, Thompson JV. Pathogenesis of adverse drug reactions. In: Davies DM, editor. Textbook of Adverse Drug Reactions. Oxford: Oxford University Press; 1977. pp 10.

8. https://www.eaaci.org/attachments/669_Italian-ENDAQuestionnaire Data ultima consultazione on line 03/02/2019.

9. http://www.agenziafarmaco.gov.it/sites/default/files/ Scheda_elettronica_AIFA_operatore_sanitario_25.09.2 013.doc Data ultima consultazione on line 03/02/2019.

10. www.agenziafarmaco.gov.it/content Data ultima consultazione on line 03/02/2019.

11. American Geriatrics Society 2019 Beers Criteria Update Expert Panel. American Geriatrics Society updated AGS Beers Criteria for potentially inappropriate medication use in older adults. J Am Geriatr Soc 2019 [Epub ahead of print].

12. The Story of Goldilocks and the Three Bears. Available from: https://www.dltk-teach.com/rhymes/goldilocks story.htm Accessed: October 30, 2018.

13. AGS Expert Panel on the Care of Older Adults with Multimorbidity. Guiding principles for the care of older adults with multimorbidity: an approach for clinicans: American Geriatrics Society Expert Panel on the Care of Older Adults with Multimorbidity. J Am Geriatr Soc 2012;60:E1-25.

14. Steinman MA, Beizer JL, DuBeau CE, et al. How to use the American Geriatrics Society 2015 Beers Criteria a guide for patients, clinicians, health systems, and payors. J Am Geriatr Soc 2015;63:e1-e7.

15. Steinman MA, Beizer JL, DuBeau CE, et al. How to use the American Geriatrics Society 2015 Beers Criteria - A guide for patients, clinicians, health systems, and payors. The Journal of the American Geriatrics Society. 
16. Garattini S, Nobili A. Interazione tra farmaci. Istituto di Ricerche Farmacologiche Mario Negri. Pavia: Selecta Medica; 2003.

17. https://www.torrinomedica.it/farmaci/interazioni/CercaInterazioni.asp

18. Interazioni in: Guida all'uso dei farmaci 2. Ministero della Salute. Milano, Masson Spa 2003: 549-602. www.guidausofarmaci.it/pag21000.htm
19. https://www.webmd.com/drugs/2/drug-5190

20. https://www.hep-druginteractions.org/checker

21. O'Connor MN, O 'Sullivan D, et al. Prevention of hospital-acquired adverse drug reactions in older people using screening tool of older persons' prescriptions and screening tool to alert to right treatment criteria: a cluster randomized controlled trial. J Am Geriatr Soc 2016. 22. https://clinicalweb.marionegri.it/intercheckweb/ 


\section{Mobilizzazione dei pazienti}

Giorgia Querci, Adele Spadaro

SC Medicina Interna, ASL 5 Liguria, La Spezia, Italia

\section{Introduzione}

L'esigenza di un protocollo per una mobilizzazione precoce e sicura dei pazienti delle nostre corsie nasce dalla volontà di evitare situazioni con possibili ripercussioni socio-economiche sia sui famigliari che sul personale medico-infermieristico e di supporto. Alcuni esempi potrebbero essere: riduzione o perdita della preesistente autonomia dei pazienti; complicanze legate alla sindrome da allettamento; allungamento del tempo di degenza; possibili cadute in reparto correlabili ad una mobilizzazione tardiva/inadeguata. Tale protocollo potrebbe essere gestito dal personale infermieristico e di supporto con la supervisione del personale medico avente il compito di verificare eventuali indicazioni/controindicazioni alla mobilizzazione ed adeguare il piano di mobilizzazione alle condizioni cliniche del paziente. ${ }^{1,2}$

È oggetto di interesse di tale protocollo qualunque paziente ricoverato per patologia medica acuta avente (Tabella 1): i) uno stato neurologico adeguato (agitazione psicomotoria assente, capacità di comprendere istruzioni, volontà da parte del paziente di essere mobilizzato e assenza di controindicazioni dettate dalla presenza di ipertensione endocranica o di sospetto danno neurologico da trauma a carico della colonnacerebrale); ii) una stabilità emodinamica (pressione arteriosa e frequenza cardiaca adeguate, assente terapia con inotropi o vasopressori, assenza di sanguinamento attivo) e un buon compenso respiratorio. Bisogna prestare attenzione in caso di embolia polmonare ad elevato rischio (ad esempio in terapia eparinica ev), trombosi venosa profonda non ancora in terapia far-

Corrispondente: Giorgia Querci, Dirigente medico, ASL 5 Spezzino, Italia

E-mail: giorgia.querci@as15.liguria.it

Articolo pubblicato secondo la Creative Commons Attribution NonCommercial 4.0 License (CC BY-NC 4.0).

${ }^{\circ}$ Copyright: the Author(s), 2019

Licensee PAGEPress, Italy

QUADERNI - Italian Journal of Medicine 2019; 7(6):151-152 macologica, emodialisi intermittente, fratture instabili ed una scarsa tolleranza del paziente allo sforzo fisico.

Per pazienti con stato psichico alterato è necessario provvedere ad un'adeguata titolazione della terapia farmacologica e ad un'educazione dei famigliari per assistenza ed attuazione del piano di mobilizzazione.

\section{Materiale occorrente}

- Anamnesi relativa allo stato di mobilizzazione e autonomia nella gestione delle attività quotidiane adeguatamente compilato dal personale medico/infermieristico all'ingresso del paziente in Reparto.

- Piano di mobilizzazione giornaliero con check list contenente: i) promemoria delle indicazioni/controindicazioni alla mobilizzazione; ii) annotazione dell'attività giornalmente eseguita e se è stata adeguamente portata a termine e tollerata dal paziente.

- Disponibilità di personale infermieristico e di supporto.

- Almeno una poltrona per stanza o una sedia per degente.

- Presidi per pazienti con obesità.

Tabella 1. Checklist: indicazioni e controindicazioni alla mobilizzazione.

\begin{tabular}{l}
\hline Stato neurologico \\
\hline Assente agitazione psicomotoria \\
\hline Capacità di comprendere istruzioni semplici \\
\hline Compliance del paziente alla mobilizzazione \\
\hline Assente ipertensione endocranica \\
\hline Assente danno neurologico da trauma \\
\hline Stabilità emodinamica \\
\hline Assente pressione arteriosa $\geq 140 / 90 \mathrm{o} \leq 100 / 60 \mathrm{mmHg}$ \\
\hline Assente frequenza cardiaca $\geq 90$ o $\leq 60 \mathrm{bpm}$ \\
\hline Assente terapia con inotropi e/o vasopressori \\
\hline Assente sanguinamento in atto \\
Compenso respiratorio \\
Assente pO 2 in AA e/o ossigenoterapia $<60 \mathrm{mmHg}$ \\
e/o FR $>20$ atti/minuto
\end{tabular}


Acquisizione della posizione seduta a bordo letto per 3'
* In caso di manifesta intolleranza da parte del paziente (malessere, vertigini) in uno dei passaggi coinvolgere il personale medico per rivalutazione clinica ed eventuale revisione del piano di mobilizzazione con coinvolgimento, se necessario, di fisiatra/fisioterapista
Passaggio alla posizione eretta $\mathrm{e}$ successivamente tentativo di trasferire il paziente dal letto alla poltrona
SI

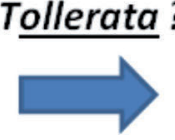

NO*

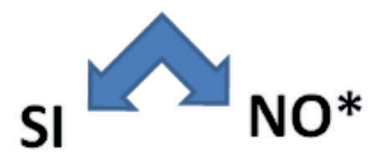

SI

\section{Deambulazione per un breve tratto}

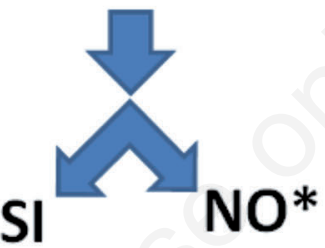

Aumentare nell'arco di una stessa giornata o delle successive il livello di mobilizzazione in modo da riportarlo allo stato antecedente il ricovero

Figura 1. Flow-Chart: piano di mobilizzazione.

\section{Modalità operative}

È necessario individuare le fasce orarie più idonee per la mobilizzazione dei pazienti, in rapporto alle attività di reparto.

In assenza di controindicazioni alla checklist, il personale infermieristico e di supporto può procedere autonomamente alla mobilizzazione graduale del paziente seguendo i seguenti passaggi (Figura 1): i) acquisizione della posizione seduta a bordo letto testando la tolleranza da parte del paziente nel mantenerla per almeno 3' senza comparsa di malessere/vertigini; ii) passaggio alla posizione eretta, successivo trasferimento dal letto alla poltrona e in caso che quest'ultimo passaggio avvenga con l'aiuto al massimo di 2 persone tentativo di fare deambulare il paziente per un breve tratto; iii) aumento nell'arco di una stessa giornata o delle successive il livello di mobilizzazione del paziente in modo da riportarlo allo stato antecedente il ricovero.
In caso di controindicazioni alla check list e/o manifesta intolleranza da parte del paziente (malessere, vertigini) in uno dei passaggi, è necessario coinvolgere il personale medico per rivalutazione clinica ed eventuale revisione del piano di mobilizzazione.

Per i pazienti meno collaboranti è necessario coinvolgere i familiari nel piano di mobilizzazione, richiedendone la presenza per tutta la durata.

\section{Bibliografia}

1. Drolet A, DeJuilio P, Harkless S, et al. Move to improve: the feasibility of using an early mobility protocol to increase ambulation in the intensive and intermediate care settings. Phys Ther 2013;93:197-207.

2. The SAFEMOB Task Force. Safe prescription of mobilizing patients in acute care settings - What to assess, what to monitor, when not to mobilize and how to mobilize and progress. Available from: https://www.physiopedia.com/SAFEMOB 


\title{
Il rischio clinico: conclusioni
}

\author{
Andrea Fontanella \\ Presidente Nazionale FADOI, Direttore del Dipartimento di Medicina e Pronto Soccorso, Ospedale Buon Consiglio \\ Fatebenefratelli, Napoli, Italia
}

A ogni accrescimento della conoscenza e della tecnica, la saggezza diviene più necessaria, poiché ognuno di questi accrescimenti aumenta la nostra capacità di attuare i nostri scopi, e perciò aumenta la nostra capacità di far del male, se i nostri scopi non sono saggi. ${ }^{1}$ Ho scelto questa frase, scritta da Bertrand Russel nel 1956, in un suo memorabile saggio su conoscenza e saggezza, perché essa da sola rappresenta, anche in ambito medico, l'essenza dell'evoluzione tecnica e antropologica della nostra società, con la conseguenza di potere ottenere da un lato grandi risultati in termini di miglioramento della qualità di vita $\mathrm{e}$ di gestione delle patologie, dall'altro di potere determinare grande danno per i pazienti se queste conoscenze e capacità tecniche non sono gestite con la dovuta appropriatezza, cautela, precisione e saggezza applicativa.

Il rischio è la potenzialità che un'azione o un'attività scelta (includendo la scelta di non agire) porti a una perdita o a un evento indesiderabile. La nozione implica che una scelta influenzi il risultato. Sebbene ogni comportamento umano sia rischioso, alcuni hanno una percentuale di rischio maggiore. Quello del rischio è un concetto connesso con le aspettative umane e la loro capacità di predizione/intervento in situazioni non note o incerte. Indica un potenziale effetto su un bene che può derivare da determinati processi in corso o da determinati eventi futuri. Ecco, quindi, che quello medico è certamente l'ambito in cui il nostro comportamento e le nostre scelte possono determinare i maggiori danni, perché si realizzano in un contesto, quello biologico, in cui la prevedibilità degli

Corrispondente: Andrea Fontanella, Presidente Nazionale FADOI, Direttore del Dipartimento di Medicina e Pronto Soccorso, Ospedale Buon Consiglio Fatebenefratelli, via Manzoni 220, 80124 Napoli, Italia.

E-mail: andreafontanella52@gmail.com

Articolo pubblicato secondo la Creative Commons Attribution NonCommercial 4.0 License (CC BY-NC 4.0).

${ }^{\circ}$ Copyright: the Author(s), 2019

Licensee PAGEPress, Italy

QUADERNI - Italian Journal of Medicine 2019; 7(6):153-156 eventi è al di fuori di qualunque logica cartesiana, ed è rivolto a popolazioni fragili, frequentemente in condizioni critiche, con le più diverse possibilità evolutive e spesso in circostanze in cui le decisioni devono essere prese con velocità e sotto la spinta delle più diverse situazioni emotive.

Il secondo aspetto che incrementa il rischio clinico, oltre quello dell'eccessiva velocità di evoluzione della tecnica rispetto al pensiero, è stato, nell'ambito della Sanità pubblica, il processo di cambiamento, sia di tipo procedimentale (modifiche dell'iter, dei requisiti di accesso, passaggi di competenze, modalità di retribuzione, etc.) che di tipo organizzativo (decentramento, contratto di lavoro, controllo di gestione, trasparenza e accesso, etc.). Questo cambiamento normativo è solo un sintomo evidente di un processo evolutivo molto più ampio: la Pubblica Amministrazione è chiamata a ripensare il suo ruolo nella collettività perché la distanza tra istituzioni e cittadini rischia di diventare insostenibile. A tal proposito non si possono trascurare i seguenti punti storici: dal secondo dopoguerra vi è stato un progressivo allontanamento dagli obiettivi misurabili. La spesa pubblica è stata considerata come motore di sviluppo economico. Di conseguenza lo strumento di tale fattore è stato un'articolazione burocratica fine a se stessa. Con l'avvento dell'Unione Europea e la globalizzazione dei mercati vi è stata una saturazione dei sistemi economici occidentali. Diviene, quindi, indispensabile razionalizzare la gestione dell'assistenza sanitaria per poterla rendere fattibile e competitiva. È, inoltre, necessario puntare alla soddisfazione dei bisogni di tutti gli attori che concorrono allo sviluppo dell' assistenza: personale sanitario medico, paramedico, amministrativo, cittadini. L'invecchiamento della popolazione è associato a un progressivo maggior assorbimento delle risorse dalle fasce di età superiori ai 65 anni e a un sempre minor numero di popolazione attiva che finanzia la sanità. Il problema è quindi se si riuscirà, nonostante questo trend d'incremento della spesa, a mantenere sostenibile il sistema sanitario. Tutto questo ha portato nel tempo ad una sempre maggiore necessità di realizzare tagli alla spesa pubblica ed alla Sanità in particolare. Tra i primi vi è una riduzione dei posti letto per acuti negli ospedali, questi scendono al di 
sotto di quattro $(3,7)$ per ogni mille abitanti, comprensivi di 0,7 (/1000 ab.) per la riabilitazione e la lungo degenza post-acuzie.

Questi tagli sono giustamente dettati dalla necessità di razionalizzare la spesa pubblica, migliorandone l'efficienza, adeguando il sistema assistenziale ai mutamenti sociali ed epidemiologici, che vedono una società sempre più anziana, affetta da polipatologie, tutte croniche, con necessità di assistenza continuativa, con perdita di autonomie. La maggioranza delle famiglie non è in grado di erogare tale assistenza, con le conseguenti dimissioni difficili e frequenti e precoci reospedalizzazioni. In quest'ambito la Medicina Interna ha una funzione portante, coordinando e completando il livello di complessità metaspecialistica e rivestendo la primaria missione di governo clinico.

In questo senso la FADOI ha un notevole vantaggio competitivo, avendo da tempo avviato e realizzato imponenti piani formativi nell'ambito del management in Sanità. Naturalmente la clinical governance impone a tutti i professionisti sanitari sia si acquisire nuove competenze - non ancora previste dai programmi di formazione universitaria e specialistica sia soprattutto di essere accountable in tutte le prescrizioni diagnostico-terapeutico-assistenziali, rinunciando ad autonomie professionali e interessi corporativi, oggi non più sostenibili.

Ecco il motivo per cui questo numero monografico, dedicato al rischio clinico ed alla sua gestione, ha assunto un aspetto multifattoriale, proprio per affrontare le mille facce del Rischio in Sanità: farmacologico, assistenziale, gestionale, economico, sociale, epidemiologico, antropologico, strutturale... cercando di coinvolgere tutti gli attori di questo sistema complesso.

Un posto preminente è stato naturalmente occupato dalla corretta gestione farmacologica, trattato in modo generale, sotto l'aspetto gestionale, da Elisa Romano, nella sua qualifica di Clinical Risk Manager, oltre che essere una degli Editor di questo numero dei Quaderni. Si è partiti dalle raccomandazioni ministeriali di sicurezza nell'uso dei farmaci per arrivare alla ricognizione e riconciliazione della terapia. Nello specifico, poi, tra i tanti farmaci usati nella Medicina Interna, è evidente come si sia voluto affrontare la corretta gestione delle terapie che hanno avuto la maggiore evoluzione innovativa e che, proprio per la loro efficacia, hanno la maggiore probabilità di arrecare danno: la terapia ipoglicemizzante e quella anticoagulante. Si è, quindi, necessariamente trattato degli strumenti per una gestione sicura delle allergie a farmaci e delle interazioni farmacologiche. Due brillanti articoli di Micaela La Regina sono dedicati ad uno degli aspetti distintivi dell'evoluzione verificatasi nelle Medicine Interne: la corretta gestione dell'ossigeno terapia e della ventilo terapia.

Dai problemi della terapia si passa poi a quelli della prevenzione delle infezioni, del trombo-embolismo venoso, delle cadute, degli errori nell'identificazione del paziente, ma anche di casi particolari, come atti autolesivi, vagabondaggio, intrappolamento nel paziente ospedalizzato.

Il fondamentale coinvolgimento infermieristico nella gestione del rischio clinico è stressato nei due articoli uno sull'importanza degli alti livelli di staffing infermieristico, che risultano essere positivamente correlati agli esiti assistenziali, e l'altro dedicato al ruolo dell'infermiere nella mobilizzazione precoce e sicura dei pazienti delle nostre corsie, anche allo scopo di evitare situazioni con possibili ripercussioni socioeconomiche sia sui famigliari sia sul personale medico-infermieristico e di supporto.

Non possono poi mancare considerazioni sul ruolo delle competenze non tecniche (non technical skill) nella dinamica del rischio clinico, questo perché nell'ambito della gestione del rischio le risorse umane rappresentano, infatti, sia una fondamentale risorsa sia una criticità.

L'ospedale è un sistema complesso in cui le attività lavorative sono altamente interdipendenti, richiedendo un alto grado di coordinamento tra i diversi gruppi professionali. Le persone che rivestono un ruolo importante nell'organizzazione sono altamente professionalizzate e generalmente manifestano primariamente lealtà alla categoria professionale di appartenenza piuttosto che all'organizzazione in cui lavorano. ${ }^{2}$ Allo scopo di potere favorire il lavoro comune, per promuovere la sicurezza del paziente, si è voluta sentire l'opinione di un Direttore Generale, di un direttore Sanitario, entrambi Internisti, e di un Direttore di Medicina Interna, i risultati a confronto sono stati estremamente interessanti.

Non potevano poi mancare alcuni spunti di riflessione sui rapporti tra rischio clinico e carico di lavoro, argomento che si correla strettamente con l'altro serio problema che è quello della gestione dei pazienti in appoggio, tema, quest'ultimo, su cui la FADOI sta lavorando intensamente con un articolato progetto di ricerca. In una possibile proiezione futura, anche allo scopo di risolvere il cronico problema del sovraffollamento dell'ospedale, viene proposta la possibilità di innovazioni organizzative in Medicina Interna per la sicurezza del paziente: dall'Ambulatorio Integrato Diagnostico-Terapeutico alla Sezione di Decisione Clinica.

A chiusura di questa mia carrellata, che ha voluto percorrere tutti gli spunti di questo numero dei Quaderni, non posso non citare quello che appropriatamente è stato definito come la metà oscura del rischio clinico: i sinistri in medicina interna. Ovvero i contenziosi medico-legali cui i Quaderni, sotto la guida di Paola Gnerre, hanno dedicato un altro numero monografico ${ }^{3}$ ed a cui si rimanda per maggiori approfondimenti.

La conclusione vera di questa poliedrica e com- 
plessa varietà di aspetti è che la reale gestione del rischio clinica in Sanità è basata su due cardini fondamentali: i) l'appropriatezza: diagnostica, preventiva, terapeutica, assistenziale, gestionale, organizzativa, appropriatezza in ricovero e in dimissione; ii) la $\mathrm{co}-$ municazione: tra direttore e la sua equipe, tra $\mathrm{i}$ componenti della stessa e quelli degli altri reparti, tra medico e il personale sanitario tutto e, soprattutto, con il paziente e i familiari, permettendo una piena comunicazione e comprensione tra le varie figure consentendo così una corretta gestione di tutto il sistema.

\section{Bibliografia}

1. Bertrand Russel. Portraits from memory and other essays. "Knowledge and Wisdom". London: G. Allen \& Unwin; 1956.

2. Shortell SM, Kaluzny AD. Healt care management. A text in organization theory and behavior. New York, NY: John Wiley \& Sons; 1988.

3. Fontanella A, Gnerre P, Nardi R, eds. La responsabilità professionale medica oggi. Quaderni Ital J Med 2019;7(3):1-98. 


\section{LINEE GUIDA PER GLI AUTORI}

I Quaderni dell 'Italian Journal of Medicine (Quaderni ITJM), costituiscono una collana supplementare solo online annessa alla rivista Italian Journal of Medicine contenente lavori solo in lingua italiana.

I Quaderni ITJM pubblicano:

- Monografie ad hoc individuate dal Presidente FADOI, dal Consiglio Direttivo, dal Board Scientifico o dall'Editor in Chief dell'Italian Journal of Medicine, in funzione del contesto scientifico-istituzionale attuale.

- Monografie ad hoc su temi di particolare rilevanza scientifica a cura della Commissione FADOI Giovani.

- Traduzioni in italiano di alcuni lavori pubblicati sui numeri standard dell'Italian Journal of Medicine, di particolare interesse per la comunità scientifica.

\section{STESURA DEI LAVORI}

I lavori dovranno essere redatti in modo conforme alle linee guida sotto riportate:

- I manoscritti devono essere scritti interamente in lingua italiana, su documento di Word, con carattere Times New Roman/Arial, dimensione 12, formato A4, interlinea doppia e margini $2,54 \mathrm{~cm}$

Parole totali: $\max$ 4000; Sommario/Abstract: $\max 250$ parole; Bibliografia: min 40 voci; Tabelle e Figure: $3 / 5$ totali (le tabelle non devono superare n. 1 pagina del documento in Word).

- La strutturazione del contenuto deve attenersi agli standard internazionali per la Rassegna (Review): i) Abstract riassuntivo dell'intero lavoro; ii) Introduzione al tema trattato; iii) Criteri e strumenti di ricerca (criteri di inclusione/esclusione, banche dati consultate, ...); iv) i successivi paragrafi devono illustrare le più recenti scoperte scientifiche nel settore; v) Conclusioni; vi) Bibliografia.

- La prima pagina deve riportare: i) titolo (in stampatello minuscolo), senza acronimi; ii) nome e cognome per esteso di ciascun autore; iii) affiliazione(i) di ciascun autore, numerate con numeri arabi iv) eventuali ringraziamenti; v) nome e indirizzo postale completi dell'autore corrispondente, corredati da telefono, fax, e-mail; vi) da 3 a 5 parole chiave, separate da virgola. La seconda pagina può riportare: i) contributi degli autori, e.g. informazioni relative a contributi sostanziali delle persone coinvolte nello studio (http://www.icmje.org/\#author); ii) dichiarazione relativa a potenziali conflitti d'interesse; iii) ulteriori informazioni (e.g. fondi, esposizioni durante conferenze...).

- In caso di utilizzo di tabelle, queste devono essere tutte numerate con numeri arabi e citate nel testo in ordine consecutivo (e.g. NON nominare le tabelle come Tabella $1 \mathrm{~A}, 1 \mathrm{~B}, \ldots$ o $1.0,1.1, \ldots$ ). Le tabelle devono essere presentate in formato editabile. Ciascuna tabella deve essere corredata da una breve didascalia; in caso di abbreviazioni, riportare una nota a piè di CIASCUNA tabella che spieghi TUTTE le abbreviazioni presenti in ognuna.

- In caso di utilizzo di figure, queste devono essere inviate in formato tiff o .jpg, allegate al manoscritto in singoli files, secondo le seguenti specifiche:

i) a colori (salvate in modalità CMYK): minimo 300 dpi di risoluzione;

ii) in bianco e nero: minimo $600 \mathrm{dpi}$ di risoluzione;

iii) minimo $17,5 \mathrm{~cm}$ di larghezza.

Ciascuna figura deve essere corredata da una breve didascalia.

$N B$ : In caso di Tabelle/Figure riprese e/o modificate da altri lavori già pubblicati, sarà cura degli autori accertarsi se tali materiali siano o meno coperti da copyright e procurarsi i permessi necessari per la riproduzione. Tali permessi dovranno essere allegati alla versione definitiva del lavoro. L'ufficio editoriale si riserva la facoltà di rimuovere Tabelle/Figure coperte da copyright, se sprovviste dei necessari permessi.

- In caso di utilizzo di abbreviazioni, la prima volta che esse sono citate è necessario scrivere per esteso la definizione+abbreviazione tra parentesi tonde [e.g. risonanza magnetica $(\mathrm{RMN})]$, a seguire si dovrà riportare solo l'abbreviazione (unica eccezione: nei titoli e nelle didascalie di tabelle e figure NON si utilizzano abbreviazioni).

\section{BIBLIOGRAFIA}

Le voci bibliografiche devono essere formattate secondo lo stile Vancouver.

Nella sezione Bibliografia, le voci bibliografiche devono essere numerate consecutivamente nell'ordine in cui appaiono per la prima volta nel testo (NON in ordine alfabetico) e, nel testo, devono essere indicate con numeri arabi in apice. Voci bibliografiche riferite a comunicazioni personali o dati non pubblicati devono essere incorporate nel testo e NON inserite tra le voci numerate [e.g. (Wright 2011, dati non pubblicati) o (Wright 2011, comunicazione personale)]. Le voci bibliografiche nella sezione Bibliografia devono tassativamente essere preparate come segue:

i) più di 3 autori, citare 3 autori, et al. Se il lavoro contiene solo 4 autori, citarli tutti e 4 ;

ii) titolo del lavoro in stampatello minuscolo;

iii) nome della rivista, senza punti, abbreviato secondo gli standard internazionali; in caso di dubbi sulla corretta abbreviazione, fare riferimento ai seguenti siti:

a. ISI Journal Abbreviations Index (http://library.caltech.edu/ reference/abbreviations/);

b. Biological Journals and Abbreviations (http://home.ncifcrf. gov/research/bja/);

c. Medline List of Journal Titles (ftp://ftp.ncbi.nih.gov/pubmed/J_Medline.txt);

iv) inserire l'anno di pubblicazione subito dopo il nome della rivista, seguito da punto e virgola;

v) NON inserire giorno o mese di pubblicazione;

vi) citare solo il volume, seguito dai due punti (NON citare il fascicolo tra parentesi);

vii) abbreviare le pagine, e.g. 351-8

Per accertarsi di aver correttamente formattato le voci bibliografiche, confrontarle con le citazioni in PubMed (http://www.ncbi. nlm.nih.gov/pubmed).

Esempi (prestare attenzione anche alla punteggiatura):

Articolo standard su Rivista

Halpern SD, Ubel PA, Caplan AL. Solid-organ transplantation in HIV-infected patients. N Engl J Med 2002;347:284-7.

Proceedings

Christensen S, Oppacher F. An analysis of Koza's computational effort statistic for genetic programming. In: Foster JA, Lutton E, Miller J, Ryan C, Tettamanzi AG, eds. Genetic programming. EuroGP 2002: Proceedings of the 5th European Conference on Genetic Programming, 2002 Apr 3-5, Kinsdale, Ireland. Berlin: Springer; 2002. pp 182-91.

Articoli i cui autori sono Organizzazioni

Diabetes Prevention Program Research Group. Hypertension, insulin, and proinsulin in participants with impaired glucose tolerance. Hypertension 2002;40:679-86.

Libri

Murray PR, Rosenthal KS, Kobayashi GS, Pfaller MA. Medical microbiology. 4th ed. St. Louis, MO: Mosby; 2002. (CITAZIONE DEL LIBRO INTERO)

Meltzer PS, Kallioniemi A, Trent JM. Chromosome alterations in human solid tumors. In: Vogelstein B, Kinzler KW, eds. The genetic basis of human cancer. New York, NY: McGraw-Hill; 2002. pp 93113. (CITAZIONE DI UN CAPITOLO)

\section{MODALITÀ D'INVIO DEI LAVORI}

Monografie

Gli Autori dovranno fare riferimento all'Editor-in-Chief o alle persone da lui designate nelle lettere di invito a scrivere gli articoli programmati.

Per gli articoli inviati da giovani internisti, gli Autori dovranno fare riferimento alla Dr.ssa Paola Gnerre (e-mail: pgnerre@yahoo.it) e agli eventuali altri referenti da lei designati, nelle prime fasi di stesura dei manoscritti, revisioni e correzioni. 


\section{QUADERNI}

dell'Italian Journal

of Medicine

La Dr.ssa Gnerre raccoglierà poi le versioni definitive dei lavori di ciascuna monografia e provvederà all'invio di tutti i materiali all'ufficio editoriale.

I lavori solo nella loro versione definitiva e approvata dalla Commissione FADOI Giovani dovranno pervenire all'ufficio editoriale già pronti per l'impaginazione e immediata pubblicazione (già corredati da eventuali permessi per la riproduzione di tabelle e immagini redatti secondo le presenti linee guida).

Traduzioni

Previo invito dell'Editor-in-Chief, gli Autori dovranno far pervenire all'ufficio editoriale la versione tradotta in italiano, al seguente indirizzo e-mail: paola.granata@pagepress.org

Il file in formato Word dovrà essere formattato secondo gli standard editoriali della rivista ufficiale ed essere già pronto per impagina- zione e immediata pubblicazione (corredato da eventuali permessi per la riproduzione di tabelle e immagini).

Si prega di inviare le eventuali tabelle in formato editabile e le figure in alta definizione secondo gli standard sopra riportati.

\section{NOTA PER GLI AUTORI}

I lavori pubblicati sui Quaderni ITJM non verranno indicizzati, ma saranno liberamente disponibili in un'apposita sezione del sito FADOI (http://www.fadoi.org/) e della rivista ufficiale.

Gli Autori i cui lavori siano accettati per la pubblicazione sui Quaderni ITJM e che fossero interessati a vederli pubblicati anche sulla rivista ufficiale, dovranno sottomettere attraverso il sito dell'ITJM (www.italjmed.org) la versione (già tradotta) in inglese e redatta in modo conforme alle linee guida della rivista; seguiranno poi la procedura di selezione tramite peer review e, se accettati, saranno inseriti nel piano editoriale standard.

\section{STAFF EDITORIALE}

Paola Granata, Journal Manager

paola.granata@pagepress.org

Claudia Castellano, Production Editor

Tiziano Taccini, Technical Support
PUBBLICATO DA

PAGEPress Publications

via A. Cavagna Sangiuliani 5

27100 Pavia, Italy

T. +39.0382 .464340$

F: +39.0382 .34872$

\section{agepress}

www.pagepress.org info@pagepress.org

\section{QUADERNI - ITALIAN JOURNAL OF MEDICINE}

Tutti gli articoli pubblicati sui QUADERNI - Italian Journal of Medicine sono redatti sotto la responsabilità degli Autori. La pubblicazione o la ristampa degli articoli della rivista deve essere autorizzata per iscritto dall'editore. Ai sensi dell'art. 13 del D.Lgs 196/03, i dati di tutti i lettori saranno trattati sia manualmente, sia con strumenti informatici e saranno utilizzati per l'invio di questa e di altre pubblicazioni e di materiale informativo e promozionale. Le modalità di trattamento saranno conformi a quanto previsto dall'art. 11 del D.Lgs 196/03. I dati potranno essere comunicati a soggetti con i quali PAGEPress intrattiene rapporti contrattuali necessari per l'invio delle copie della rivista. Il titolare del trattamento dei dati è PAGEPress Srl, via A. Cavagna Sangiuliani $5-27100$ Pavia, al quale il lettore si potrà rivolgere per chiedere l'aggiornamento, l'integrazione, la cancellazione e ogni altra operazione di cui all'art. 7 del D.Lgs 196/03.

https://www.italjmed.org/index.php/ijm/quad 
PAGEPress - Scientific Publications

Via A. Cavagna Sangiuliani, 5 - 27100 Pavia - Italy

Phone +390382 464340 - Fax +39038234872

www.pagepress.org 